\title{
Laboratory Directed Research and Development Annual Report
}

Fiscal Year 1993

January 1994

Prepared for the U.S. Department of Energy under Contract DE-AC06-76RLO 1830

Pacific Northwest Laboratory

Operated for the U.S. Department of Energy by Battelle Memorial Institute 


\title{
DISCLAIMER
}

This report was prepared as an account of work sponsored by an agency of the United States Government. Neither the United States Government nor any agency thereof, nor Battelle Memorial Institute, nor any of their employees, makes any warranty, expressed or implied, or assumes any legal liability or responsibility for the accuracy, completeness, or usefulness of any information, apparatus, product, or process disclosed, or represents that its use would not infringe privately owned rights. Reference herein to any specific commercial product, process, or service by trade name, trademark, manufacturer, or otherwise does not necessarily constitute or imply its endorsement, recommendation, or favoring by the United States Government or $a_{1 .}$, agency thereof, or Battelle Memorial Institute. The views and opinions of authors expressed herein do not necessarily state or reflect those of the United States Government or any agency thereof.

\author{
PACIFIC NORTHWEST LABORATORY \\ operated by \\ BATTELLE MEMORIAL INSTITUTE \\ for the \\ UNITED STATES DEPARTMENT OF ENERGY \\ under Contract DE-AC06-76RLO 1830
}

Printed in the United States of America

Available to DOE and DOE contractors from the

Office of Scientific and Technical Information, P.O. Box 62, Oak Ridge, TN 37831; prices available from (615) 576-8401. FTS 626-8401.

Available to the public from the National Technical Information Service,

U.S. Department of Commerce, 5285 Port Royal Rd., Springfield, VA 22161. 


\section{Laboratory Directed Research and Development Annual Report}

Fiscal Year 1993

January 1994

Prepared for

the U.S. Department of Energy

under Contract DE-AC06-76RLO 1830

Pacific Northwest Laboratory

Richland, Washington 99352 


\section{Contents}

Introduction $\ldots \ldots \ldots \ldots \ldots \ldots \ldots \ldots \ldots \ldots \ldots \ldots \ldots \ldots \ldots \ldots \ldots \ldots \ldots$

Overview and Management Process $\ldots \ldots \ldots \ldots \ldots \ldots \ldots \ldots \ldots \ldots \ldots \ldots \ldots$

1.0 Laboratory Level Projects

1.1 Integrated Environmental Research

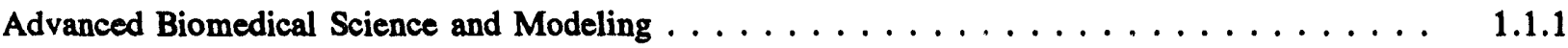

Advanced Methods and Analytical Technology $\ldots \ldots \ldots \ldots \ldots \ldots \ldots \ldots \ldots$

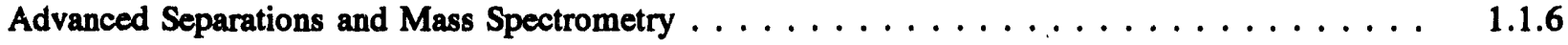

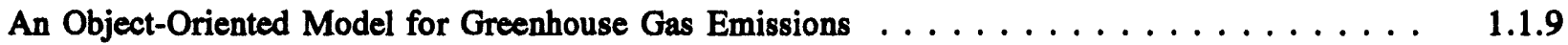

Analysis of Non-Statutory Mechanisms for Implementation of Global Environmental

Policies: Methodology Development for Public Policy and Industrial Information Needs . . . . .

Characterization of Structure and Dynamics of Surface Adsorbates and Their Surfaces . . . . 1.1.13

Chemical Adducts and Radiation Sensitivity of Supercoiled DNA $\ldots \ldots \ldots \ldots \ldots \ldots \ldots$

Chemical Nesorption/Dissolution Kinetics $\ldots \ldots \ldots \ldots \ldots \ldots \ldots \ldots \ldots \ldots$

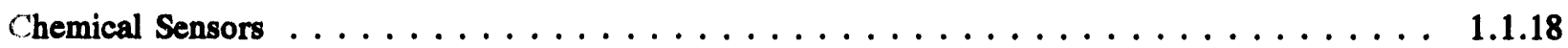

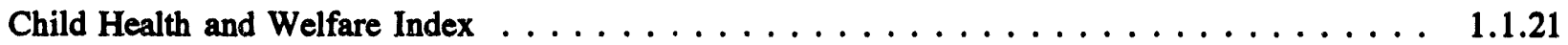

Computational Modeling of Complex Physical Systems $\ldots \ldots \ldots \ldots \ldots \ldots \ldots \ldots$

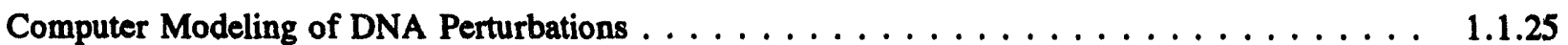

Contaminant Risk Evaluation and Management $\ldots \ldots \ldots \ldots \ldots \ldots \ldots \ldots \ldots$

Determination of the Potential for Using Genetic and Physical Biomarkers of the Big

Sagebrush as a Tool for Investigating Environmental Change $\ldots \ldots \ldots \ldots \ldots \ldots$

Development of Advanced Flow Cytometry Methods for investigating EMF-Induced

Calcium Ion Mobility . . . . . . . . . . . . . . . . . . . . . . . . 1.1.34

Development of a Regional Scale Risk Computation Capability for Assessment of

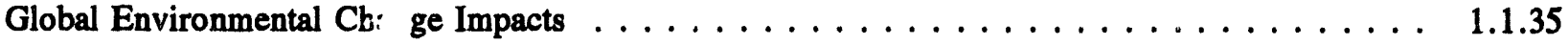

Elastic Properties, Solution Chemistry, and Electronic Structures of Oxides, Silicates, and

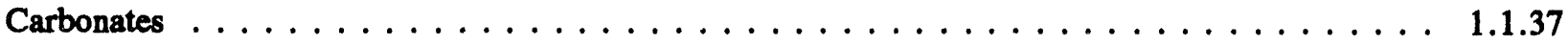

EMF Biological Effects: Characterization of Magnetic Fields in Homes . . . . . . . . . . . 1.1.39 
Enzymatic Transformation of Inorganic Chemicals

Epitaxial Growth and Surface Structure Determination of Novel Oxide Surfaces

Fisheries Consequences of Slobal Climate

Fourier Transform EPR Studies of Radiation-Induced Structural Alteration of DNA . . . . . .

Fourier Transform Ion Cyclotron Resonance Mass Spectroscopy Data Acquisition

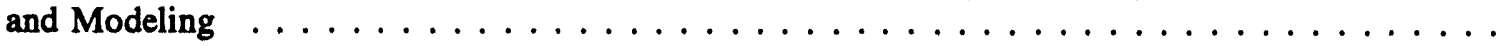

Global Environmental Decision Making $\ldots \ldots \ldots \ldots \ldots \ldots \ldots \ldots \ldots \ldots$

Graphical Interface for Ocean Global Circulation Models $\ldots \ldots \ldots \ldots \ldots$. . . . . . . . . . 1.54

Health Protection and Standards for Hazardous Chemicals $\ldots \ldots \ldots \ldots \ldots \ldots$

High Field NMR and NMR Imaging $\ldots \ldots \ldots \ldots \ldots \ldots \ldots \ldots \ldots \ldots$

Improved Analytical Ion Trapping Methods $\ldots \ldots \ldots \ldots \ldots \ldots \ldots \ldots \ldots$

Infrared Atmospheric Continuum Absorption $\ldots \ldots \ldots \ldots \ldots \ldots \ldots 1 . \ldots \ldots$

Integrated Environmental Monitoring $\ldots \ldots \ldots \ldots \ldots \ldots \ldots \ldots \ldots$

Integrated Regional Climate Change $\ldots \ldots \ldots \ldots \ldots \ldots \ldots \ldots \ldots \ldots$

Integrated Systems Ecology as an Approach for Unifying Global Change Issues $\ldots \ldots \ldots$

Integration of Molecular Research with Environmental Phenomena $\ldots \ldots \ldots \ldots$

Interphase Chemistry $\ldots \ldots \ldots \ldots \ldots \ldots \ldots \ldots \ldots \ldots \ldots \ldots \ldots \ldots$

Land Use and Agricultural Model of Global Environmental Change $\ldots \ldots \ldots \ldots \ldots$

Magnetic Resonance Spectroscopy . . . . . . . . . . . . . . . . . . . . . . . 1.1.74

Materials Surface and Interface Chemistry $\ldots \ldots \ldots \ldots \ldots \ldots \ldots \ldots \ldots$

Migration and Climatic Change: Events from the Dust Bowl and Their Implication

for the Migration of Populations into the United States $\ldots \ldots \ldots \ldots \ldots \ldots$

Molecular Dynamics at Solid Surface Interfaces . . . . . . . . . . . . . . . . . 1.1.79

Multinuclear Solid-State NMR Characterization of the Early Forms of Mineralization $\ldots \ldots$. . 1.1 .81

PBPK-Based Breath Analysis Instrumentation Demonstration $\ldots \ldots \ldots \ldots \ldots \ldots$

Principles of Design for Global Observing Networks $\ldots \ldots \ldots \ldots \ldots$. . . . . . . . . 1.1 .84

Protein Structure Analysis $\ldots \ldots \ldots \ldots \ldots \ldots \ldots \ldots \ldots \ldots \ldots \ldots$ 
Radiative Forcing by Anthropogenic Sulfate Aerosols $\ldots \ldots \ldots \ldots \ldots \ldots \ldots$

Radiometric Characterization of Subvisual Cirrus Clouds $\ldots \ldots \ldots \ldots \ldots \ldots$

Redesign of Biodegradative Enzymes: Theory Development and Coupled Theory-

Expsiment Approach $\ldots \ldots \ldots \ldots \ldots \ldots \ldots \ldots \ldots \ldots \ldots \ldots$

Remotely Piloted Vehicle for Global Climate Change Measurements . . . . . . . . . . . . 1.1 .97

Site-Specific DNA Damage by Radiation and Chemicals $\ldots \ldots \ldots \ldots \ldots \ldots \ldots$

Solid Waste Macro Material Flow Modeling $\ldots \ldots \ldots \ldots \ldots \ldots \ldots$. . . . . . . . . . . . . . .

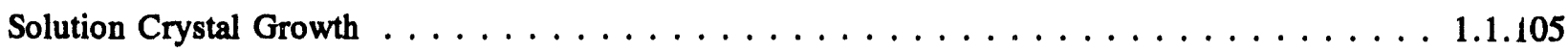

Spatial Data Visualization for Global Change $\ldots \ldots \ldots \ldots \ldots \ldots \ldots$

Spectroscopy Techniques for Analysis of DNA Damage $\ldots \ldots \ldots \ldots \ldots \ldots \ldots$

Structural and Kinetic Studies at Model Oxide Surfaces $\ldots \ldots \ldots \ldots \ldots \ldots \ldots \ldots$

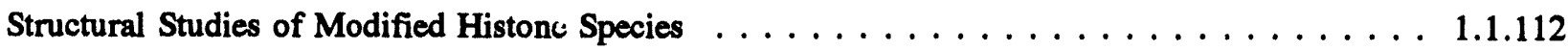

Synchrotron Research/Advanced Photon Source Beamline $\ldots \ldots \ldots \ldots \ldots \ldots \ldots$

Technological Response $\ldots \ldots \ldots \ldots \ldots \ldots \ldots \ldots \ldots \ldots \ldots \ldots \ldots 117 \ldots \ldots \ldots \ldots$

The Technical Basis for Logit Analysis Applied to Technology Markets . . . . . . . . . . . . 1.1.120

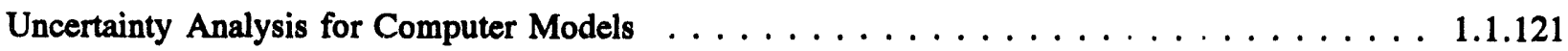

1.2 Process Technology

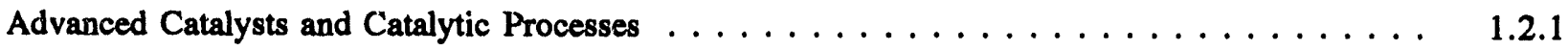

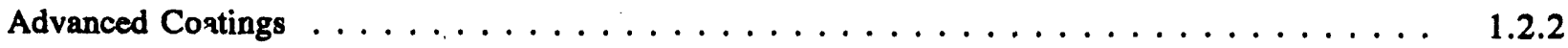

Fluid Dynamics Research $\ldots \ldots \ldots \ldots \ldots \ldots \ldots \ldots \ldots \ldots \ldots$

In Situ Monitoring of Ultrafine Particle-to-Particle Material Interactions $\ldots \ldots \ldots \ldots \ldots$. . . . 1.2 .7

Materials Evaluation $\ldots \ldots \ldots \ldots \ldots \ldots \ldots \ldots \ldots \ldots \ldots \ldots \ldots \ldots \ldots \ldots$

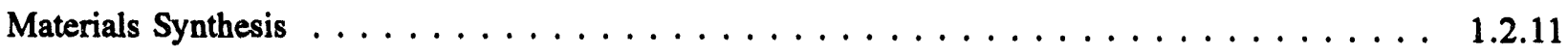

Multicenter Hazardous and Radioactive Waste Treatment and Remediation $\ldots \ldots \ldots \ldots$. . . 1.2 .13

Organic Conversion $\ldots \ldots \ldots \ldots \ldots \ldots \ldots \ldots \ldots \ldots \ldots \ldots \ldots \ldots \ldots \ldots$

Process Engineering Science $\ldots \ldots \ldots \ldots \ldots \ldots \ldots \ldots \ldots \ldots \ldots \ldots$

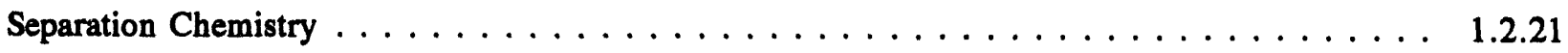




\subsection{Energy Systems Research}

Application of AI Technology to Power Plant Operations and Maintenance $\ldots \ldots \ldots \ldots \ldots$

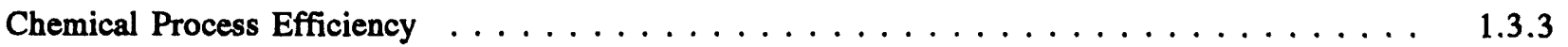

Electric Motor/Pump Diagnostician $\ldots \ldots \ldots \ldots \ldots \ldots \ldots \ldots \ldots \ldots \ldots \ldots$

General Diagnostic Reasoner $\ldots \ldots \ldots \ldots \ldots \ldots \ldots \ldots \ldots \ldots \ldots$

HVAC Diagnostic Prototype Completion $\ldots \ldots \ldots \ldots \ldots \ldots \ldots \ldots \ldots$

Integration of Economic Principles with Energy/Environmental Life-Cycle Assessment $\ldots \ldots \ldots$

Knowledge Acquisition Tools $\ldots \ldots \ldots \ldots \ldots \ldots \ldots \ldots \ldots \ldots \ldots \ldots$

Microscale Heat Exchanger and Heat Pump Architecture Development $\ldots \ldots \ldots \ldots \ldots$. . . . $\ldots 11$

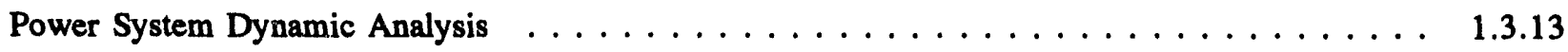

Transportation Materials $\ldots \ldots \ldots \ldots \ldots \ldots \ldots \ldots \ldots \ldots \ldots \ldots \ldots$

1.4 Other Lababoratory-Level Projects

International Germanium Experiment $\ldots \ldots \ldots \ldots \ldots \ldots \ldots \ldots \ldots \ldots \ldots$

International Reactor Safety $\ldots \ldots \ldots \ldots \ldots \ldots \ldots \ldots \ldots \ldots \ldots \ldots$

Weapons-Grade Plutonium Disposition $\ldots \ldots \ldots \ldots \ldots \ldots \ldots \ldots \ldots$

2.0 Center-Level Projects

2.1 Applied Physics

Direct Numerical and Large Eddy Simulation of Turbulent Fluid Flows . . . . . . . . . . 2.1 .1

Electrolyte Ballasting for Parallel Discharge Systems $\ldots \ldots \ldots \ldots \ldots \ldots \ldots$

Holographic Impulse Radar for Imaging Buried Waste Containers in Low Conductivity Soil . . . $\quad$ 2.1.5

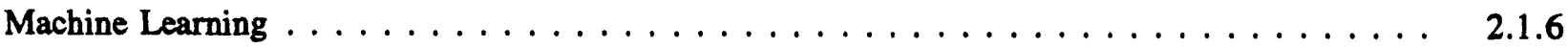

Nonlinear Constrained Optimization Models with Uncertainty $\ldots \ldots \ldots \ldots \ldots \ldots$

Portable Operations and Maintenance Information System (POMIS) $\ldots \ldots \ldots \ldots \ldots$

Probabilistic Finite Element Analysis $\ldots \ldots \ldots \ldots \ldots \ldots \ldots \ldots \ldots \ldots \ldots \ldots \ldots$

Remote Moisture Measurement by Electromagnetic Induction $\ldots \ldots \ldots \ldots \ldots \ldots \ldots$

Sensing Applications of Electrorheological Fluids $\ldots \ldots \ldots \ldots \ldots \ldots \ldots \ldots \ldots \ldots$

Sonar Impulse Target Strength Holograph $\ldots \ldots \ldots \ldots \ldots \ldots \ldots \ldots \ldots \ldots \ldots$ 
Spectroscopic Holography $\ldots \ldots \ldots \ldots \ldots \ldots \ldots \ldots \ldots \ldots$. . . . . . . . . . . . . . . . . .

Video-Based Imaging Interferometry $\ldots \ldots \ldots \ldots \ldots \ldots \ldots \ldots \ldots \ldots$

2.2 Earth and Environmental Sciences

Advances in Desktop Atmospheric Dispersion Modeling $\ldots \ldots \ldots \ldots \ldots \ldots \ldots \ldots$

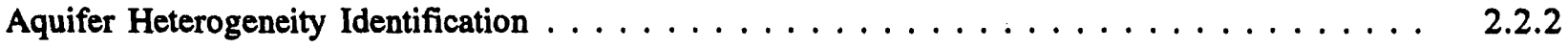

Bonding and Structural Features Controlling Reactivity on Environmental Surfaces $\ldots \ldots$

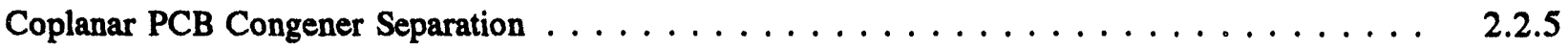

Developing a Framework for an Advanced Air Toxics Model $\ldots \ldots \ldots \ldots \ldots \ldots$

Extraction of Organic Contaminants by Supercritical Fluid Extraction $\ldots \ldots \ldots \ldots \ldots$

Modeling of In Situ Biorestoration of Organic Compounds in Groundwater Using

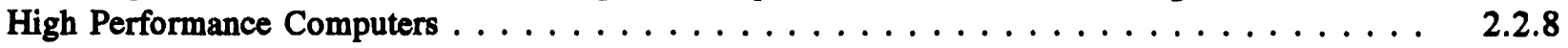

Software Development for Analyses of a New Global Meteorological Data Set . . . . . . . . 2.2 .10

\subsection{Life Sciences}

Appliance Use and Cancer $\ldots \ldots \ldots \ldots \ldots \ldots \ldots \ldots \ldots \ldots \ldots \ldots \ldots \ldots$

Assessment of Hazardous Waste Exposure and Effects $\ldots \ldots \ldots \ldots \ldots \ldots$

Cooled Optically Stimulated Luminescence in $\mathrm{Al}_{2} \mathrm{O}_{3}$ for Utrasensitive Radiation Detection $\quad \ldots \quad$ 2.3.4

Development of a Ribbon Channel Plate for DNA Sequencing $\ldots \ldots \ldots \ldots \ldots$

Ethical, Legal, and Social Implications of the Human Genome Project for Screening,

Monitoring, and Health Surveillance of Department of Energy Workers . . . . . . . . . .

Evaluation of Mercuric Iodide for Beta Radiation Detection $\ldots \ldots \ldots \ldots \ldots \ldots$

Modeling of Images of the Human Body for Applications in Absorbed Dose Calculations _. . . 2.3.11

Non-Ionizing Radiation (NIR) Hazard Assessment Software $\ldots \ldots \ldots \ldots \ldots \ldots \ldots$

Physiologically Based Pharmacokinetic Modeling of Hazardous Chemicals $\ldots \ldots \ldots$. . . . 2.3.14

Properties of Soft X-Ray Absorption and DNA Damage by Syncrotron Radiation $\ldots \ldots \ldots$

Radiation Damage and Repair in Mammary Carcinogenesis $\ldots \ldots \ldots \ldots \ldots \ldots$

Use of Proliferating Cell Nuclear Antigen (PCNA) Assay to Quantify Cell Proliferation

in Respiratory Tract of Rodents Inhaling Chemicals or Radioactive Materials . . . . . . . . . 2.3.18 
2.4 Materials and Chemical Sciences

Advanced Nanometer-Scale Electronic Materials Synthesis $\ldots \ldots \ldots \ldots \ldots \ldots$

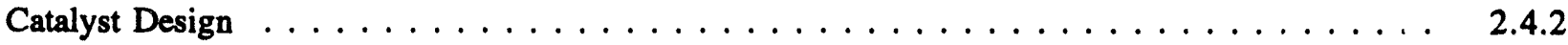

Catalyst Development for High-Temperature Pressurized Aqueous Chemical

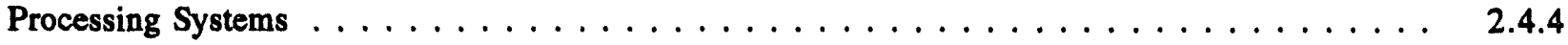

Design, Development, and Characterization of Polymer/Glass Composites $\ldots \ldots \ldots$

Development of a Selective Solid TRU Extractant $\ldots \ldots \ldots \ldots \ldots \ldots \ldots \ldots \ldots$

In Situ Composites Using Tape Casting $\ldots \ldots \ldots \ldots \ldots \ldots \ldots \ldots \ldots \ldots \ldots$

Novel Electrosynthesis of Organic Electroactive Polymers with Organo-Substituted

Cyclic Trimeric Phosphaszenes as a Dopant . . . . . . . . . . . . . . . . 2.4.11

2.5 National Security and Defense

CW Molecular Discriminator $\ldots \ldots \ldots \ldots \ldots \ldots \ldots \ldots \ldots \ldots \ldots \ldots \ldots \ldots \ldots \ldots$

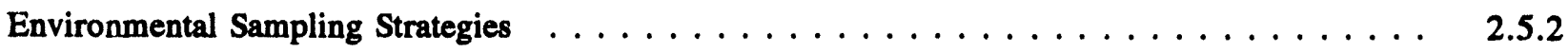

Material Structural Properties of Physics of Scintillators $\ldots \ldots \ldots \ldots \ldots \ldots$

2.6 Reactor Technology

Ceramic and Metal Matrix Composites in Nuclear Reactor Applications:

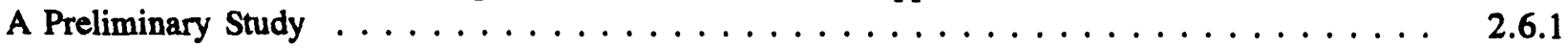

Closed Chamber Electrolyte System for Separation of Graphite from Radioactive

Materials . . . . . . . . . . . . . . . . . . . . . . . . 2.6 .3

Establishment of Experimenta' ‘'apability for Beta Alumina Work $\ldots \ldots \ldots \ldots \ldots$

2.7 Technology Planning and Analysis

Evaluating the Carcinogenic Risk of Chemical Mixtures $\ldots \ldots \ldots \ldots \ldots \ldots . \ldots \ldots$

Process Re-Engineering Support System (PRESS) $\ldots \ldots \ldots \ldots \ldots \ldots \ldots \ldots \ldots \ldots \ldots$

Proliferation Analysis Support System $\ldots \ldots \ldots \ldots \ldots \ldots \ldots \ldots \ldots \ldots \ldots \ldots$

Prototype for Integrating Total Energy Cycle and Product Life Cycle Assessments $\ldots \ldots$. . . 2.7 .6

2.8 Waste Technology

Cavitation in Supercritical Carbon Dioxide $\ldots \ldots \ldots \ldots \ldots \ldots \ldots \ldots \ldots \ldots \ldots$

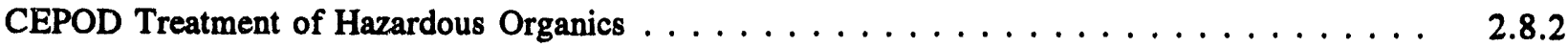

Electrokinetic Distribution of Nutrients to Enhance In Situ Bioremediation $\ldots \ldots \ldots \ldots$ 
Engineering Transport Phenomena $\ldots \ldots \ldots \ldots \ldots \ldots \ldots \ldots \ldots$

Enhanced Bioremediation Using In Situ Heating $\ldots \ldots \ldots \ldots \ldots \ldots \ldots \ldots \ldots$

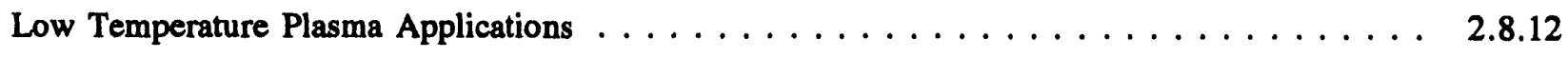

Microtechnology Applications in Waste and Chemical Processing $\ldots \ldots \ldots \ldots \ldots . \ldots$

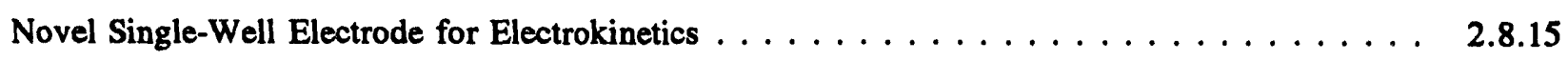

Technological Opportunities From Unproductive Waste Streams $\ldots \ldots \ldots \ldots \ldots$

Vitrification Process Rate Enhancement Using DC Plasma Arc $\ldots \ldots \ldots \ldots \ldots . \ldots \ldots$

X-Ray Film Optical Reader $\ldots \ldots \ldots \ldots \ldots \ldots \ldots \ldots \ldots \ldots \ldots \ldots$

Acronyms and Abbreviations 


\section{Introduction}

The Department of Energy Order DOE 5000.4A $\mathrm{A}^{(\mathrm{a})}$ establishes DOE's policy and guidelines regarding Laboratory Directed Research and Development (LDRD) at its multiprogram laboratories. As described in $5000.4 A, L D R D$ is "research and development of a creative and innovative nature which is selected by the Laboratory Director or his or her designee, for the purpose of maintaining the scientific and technological vitality of the Laboratory and to respond to scientific and technological opportunities in conformance with the guidelines in this Order. LDRD includes activities previously defined as ER\&D, as well as other discretionary research and development activities not provided for in a DOE program."

DOE Order 5000.4A requires that each laboratory submit an annual report on its LDRD activities to the cognizant Secretarial Officer through the appropriate Operations Office Manager. The report provided in this document represents Pacific Northwest Laboratory's (PNL) LDRD report for FY 1993.

During FY 1993, 142 LDRD projects were selected for support through PNL's LDRD project selection process. Total funding allocated to these projects was $\$ 11.8$ million. This amount represented 3\% of PNL's operating budget, which is half of the $6 \%$ maximum allowed by DOE Order $5000.4 \mathrm{~A}$.

Consistent with the Mission Statement and Strategic Plan provided in PNL's Institutional Plan, the LDRD investments are focused on developing new and innovative approaches in research related to our "core competencies." Currently, PNL's core competencies have been identified as

- integrated exvironmental research

- process technology

- energy systems research.

In this report, the individual summaries of Laboratory-level LDRD projects (presented in Section 1.0) are organized according to these core competencies. The largest proportion of Laboratory-level LDRD funds is allocated to the core competency of integrated environmental research.

A significant proportion of PNL's LDRD funds are also allocated to projects within the various research centers that are proposed by individual researchers or small research teams. Funding allocated to each of these projects is typically $\$ 35 \mathrm{~K}$ or less. The projects are described in Section 2.0 .

The projects described in this report represent PNL's investment in its future and are vital to maintaining the ability to develop creative solutions for the scientific and technical challenges faced by DOE and the nation. In accordance with DOE guidelines, the report provides an overview of PNL's LDRD program and the management process used for the program and project summaries for each LDRD project.

(a) U.S. Department of Energy Order DOE 5000.4A, Laboratory Directed Research and Development, 04-09-92. 


\section{Overview and Management Process}

The strategic importance of Laboratory Directed Research and Development (LDRD), as well as the program benefits and the Laboratory's management process are discussed in this section.

\section{Strategic Importance of Laboratory Directed Research and Development}

The relevance and value of a Department of Energy (DOE) multiprogram laboratory lies in its ability to apply science and technology to national needs that fall within the missions of the DOE. The increasing complexity of these needs and the inadequacy of using conventional approaches demand that creativity and innovation underlie scientific and technological efforts so that new and novel solutions are discovered and applied. In addition, new ideas and opportunities frequently occur at a faster pace than can be anticipated or adopted in the federal budget process.

A national laboratory must establish and maintain an environment in which creativity and innovation is encouraged and supported if it is to fulfill its missions and remain viable in the long term. For these reasons, external reviews of the DOE multiprogram laboratories have consistently recommended that laboratory directors be given discretion to select research and development projects for support and to allocate a percentage of their operating budgets to provide this support.

The LDRD program, in the long term, allows the Pacific Northwest Laboratory (PNL) to assist DOE in fulfilling its missions and contributes to other priority needs of the nation. PNL's program supports creative endeavors in areas of strategic importance to the Laboratory. PNL seeks to continually replenish its inventory of ideas that have the potential to address major national needs. The principal goals of the LDRD program are to 1) encourage the advancement of basic science and fundaniental research at the Laboratory; and 2) deveiop major, new research and development approaches. Specific objectives are to

- foster an environment that encourages creativity and innovation

- fund new and novel ideas that have scientific/technical merit but that cannot be funded promptly through programmatic channels

- investigate new ideas/concepts to the proof-of-principle stage.

The LDRD program serves to enhance the morale and vitality of the Laboratory's scientific and technical staff and to recognize their importance to the future of the Laboratory. This program has a major impact on our staff by providing a specific source of funds that can be used to promptly pursue new ideas and concepts and enrich the Laboratory's core competencies.

\section{Program Benefits}

PNL's LDRD program has provided a number of benefits relative to the generic goals of fostering creativity and innovation within the Laboratory. The program has also provided specific benefits to PNL that have allowed it to assume a major role in the development of science and technology to address significant national needs, such as the environmental restoration of DOE sites and global climate change.

When PNL became an Energy Research laboratory in 1985, one of the major DOE directives was strengthening its fundamental research capabilities. A strengthened fundamental research component would establish a sound scientific basis for PNL's applied research and development programs that would provide a complete capability for the integrated management of scientific and technical programs of national importance. 
LDRD has been the principal vehicle by which PNL has made substantial progress in improving its fundamental research base. The new capabilities developed at PNL in molecular science, high-performance computing, structural biology, and the environmental sciences have enhanced its ability to serve DOE missions. These new capabilities have changed and renewed the institutional vitality of PNL during a time when the Hanford Site was undergoing significant changes that appeared likely to undermine this vitality.

The national goal of restoring DOE sites, the total cost of which is now estimated to exceed $\$ 100$ billion, will never be accomplished in a reliable and cost-effective manner without significant contributions from science and technology. These contributions will range from developing a fundamental understanding of the effects of contaminants on molecular structure and function, to developing innovative new technologies for processing wastes. Similarly, a rational and defensible approach to addressing global environmental change will not be developed until the nature of possible changes are more fully understood and the impacts of alternative mitigating strategies are analyzed.

The LDRD program has allowed PNL to initiate the process of bringing the capabilities of the national scientific and technical community to bear on these environmental problems. These efforts are still evolving and significant challenges remain. However, many of PNL's ideas and concepts related to these areas were originally developed with LDRD funds and are now receiving programmatic support frum DOE. Examples include previous LDRDsupported work in the atmospheric sciences that is now being applied through PNL's participation in the Atmospheric Research Measurement (ARM) program, and studies in chemicai dynamics that are now being supported by the Office of Basic Energy Sciences. It is believed that current LDRD projects in advanced processing and new energy technologies will produce similar scientific and technical benefits as national efforts in these areas more fully emerge.

PNL has traditionally made a portion of its LDRD funding available to small, interdisciplinary teams of researchers with new ideas or concepts that require only a small amount of funding (typically less than \$35K) for initial testing. This practice is planned to be continued because of the significant scientific and technical benefits that have resulted. PNL's in situ vitrification (ISV) process, which is now receiving widespread testing and demonstration for use in remediating hazardous waste sites both within and outside the DOE complex was originally started through a \$5K investment in testing a new idea brought forth by one of PNL's engineering groups.

In summary, LDRD has enabled PNL to enhance its research vitality as a DOE multiprogram laboratory during a period of significant change. It has facilitated its ability to make significant contributions to addressing national problems, particularly in the environmental research area. It is essential that PNL and the other DOE laboratories retain the capability provided by LDRD if their full capacity to assist DOE and the nation is to be realized.

\section{Laboratory Directed Research and Development Management Process at Pacific Northwest Laboratory}

LDRD at PNL is funded through an overhead account applied to all technical labor hours charged to PNL's 1830 Contract accounts. Formal proposals in an authorized format are required for each LDRD project.

Decisions regarding funding allocations for LDRD accounts are made by the Laboratory Director's Office. Primary responsibility for allocations to individual LDRD projects within these accounts rests with line and technical program managers, with oversight from the Senior Science Director.

All projects are reviewed for technical merit by line managers and/or scientific staff, and in some cases by external peer reviewers. Written guidance (including a formal LDRD Guide) pertaining to the criteria and guidelines for LDRD projects provided in DOE Order 5000.4A is widely distributed to PNL staff. Adherence to these criteria is further ensured through reviews by the Office of the Director of Strategic Planning. Individual projects are usually limited to $\$ 1 \mathrm{M}$ in total funding and $\$ 500 \mathrm{~K}$ in a single fiscal year. The Director of Finance is responsible for financial oversight of the LDRD program. Accountability for individual LDRD projects rests with the principal investigators conducting the projects and their cognizant line manager. 
The major sequential steps of PNL's LDRD process are illustrated in the figure. Additional details on these steps follow

- Based on the approval of PNL's LDRD Plan by DOE, initial funding allocations for LDRD accounts are approved by the Laboratory Director and disbursed by the Director of Finance. A portion of the total LDRD funds are retained for later distribution during the fiscal year.

- Line and office managers solicit LDRD project proposals from research staff and select projects as candidates for funding. Each manager selecting an LDRD project must certify in writing that the project has been peerreviewed and meets the requirements of DOE 5000.4A.

- All LDRD project proposals and the electronic files must be submitted to PNL's LDRD Office for review for compliance with DOE requirements after the principal investigator and the cognizant manager have signed them. All documentation required to meet National Environmental Policy Act (NEPA) and other environmental, safety, and health compliance requirements for the projects must also be submitted.

- The emphasis required in the proposals is on the technical objectives and approaches that will be incorporated in the project.

- The Budget and Analysis Office has established four-digit alpha codes that are used to represent the work breakdown structure (WBS) code for each of the LDRD accounts. After a project receives final approval, a project-level work breakdown structure code is assigned by the LDRD Office for each LDRD project.

Primary responsibility for ensuring adequate technical review of LDRD projects rests with center and initiative managers. In addition, quarterly or more frequent reviews of selected projects may be performed under the leadership of the Senior Science Director. A bi-weekly LDRD seminar series also is used to review the technical status and highlights of selected projects. The various technical reviews constitute the most important means of ensuring that LDRD funds are used for their intended purpose.

Principal investigators and management are also required to develop input to meet all DOE LDRD reporting requirements. These requirements include the LDRD annual reports and the quantitative survey of LDRD project results. Selected principal investigators may also be asked to participate in annual DOE LDRD program reviews, which are organized by the Director of Strategic Planning and the LDRD Office. A group of 11 LDRD coordinators has been designated by PNL line managers to assist in managing and overseeing the LDRD program. The Director of Strategic Planning and the LDRD Office have primary responsibility for developing the annual LDRD Plan, with the concurrence of the PNL Senior Science Director and the Director of Finance.

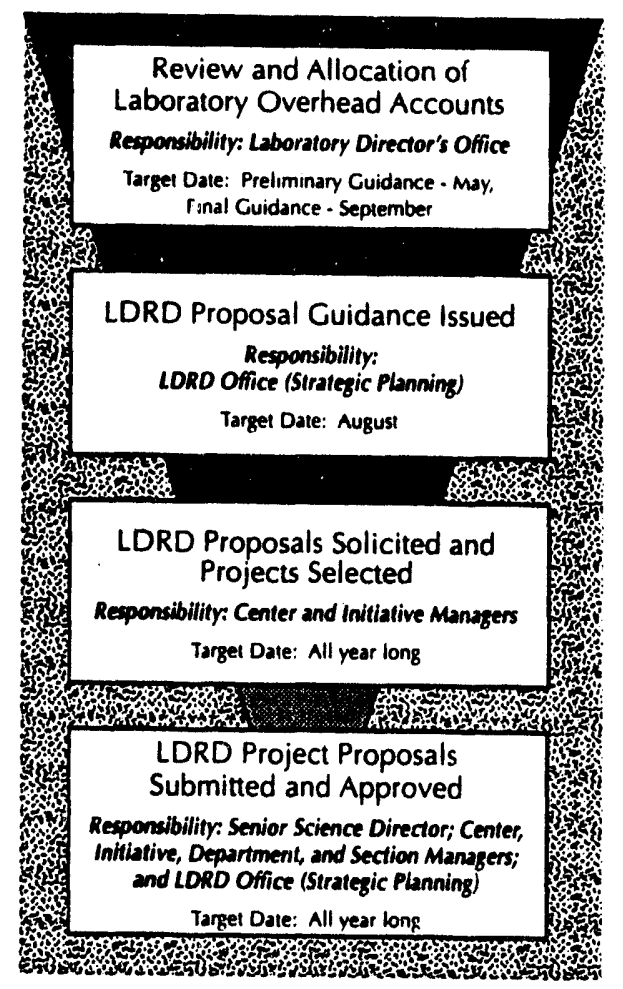

PNL's LDRD Allocation Process 


\subsection{Laboratory Level Projects}


1.1 Integrated Environmental Research 


\section{Advanced Biomedical Science and Modeling}

M. R. Sikov (Biology and Chemistry)

Based on complementary strengths throughout PNL, this project is a response to federal agencies' concerns to combine advanced technologies with the recent emphasis on molecular approaches to biomedical problems. It provided a logical progression of the U.S. Department of Energy's (DOE's) role in developing technological solutions to national concerns, and led to new means for detecting and evaluating potential health hazards associated with the work environment, as well as to enhanced diagnostic and therapeutic modalities with broad application. Integration of capabilities in physical, mathematical, and biomedical sciences, together with external collaborations, allow for substantive, innovative contributions. Specific topics addressed in the project include innovative imaging concepts, information extraction, and application of the new imaging concepts to specific biomedical research problems.

\section{Spectral Signatures for Disease Diagnosis}

This task is based on suggestions obtained from literature reviews and scientific communications that photon emission or transmission by tissues displays disease-related characteristics. Investigations have been directed toward obtaining and quantifying spectral signatures, which may be considered as images that evolve from detection or spectroscopy of intrinsic or induced fluorescence, as well as adaptive optical transmission.

Based on tissue of origin, phenotype, and growth characteristics, four representative cell lines were selected from the American Type Culture Collection (ATCC). Because of the integration of biological and physical technologies, there was a need to develop new methodologies for these investigations. Initial experiments were performed to modify routine techniques so that cell cultures could be grown under conditions compatible with ' physical requirements of available spectroscopic equipment. Subsequent efforts were directed at identification and elimination of interfering spectral signatures that were produced by the supporting matrices, cell nutrient media, and other artifacts so that the spectral properties of the cell lines could be unambiguously identified. This led to performing comparative experiments in which the effect of postculture time and the role of cell viability were evaluated.

Extensions of these studies were begun to obtain families of spectra relating to excitation wavelength, cell characteristics, and pathophysiology. Baselines were established for studies to iúsutify near-IR (infrared) autofluorescence signatures, examine the feasibility of in vivo optical detection, and search for and identify fluorophores that selectively bind to our target cell lines.

\section{Enhanced Interpretation of Radiographic and Bioelectric Images}

This phase of the study is directed at maximizing diagnostic image quality and increasing the usable content and amount of information extractable from images. Direct and indirect digitization te hniques have been used to enhance the detection and diagnosis of disease through pattern recognition procedures and/or textural analysis of images.

To date, the source material for this investigation consisted of a series of thoracic radiographs of dogs that developed lung tumors following exposure to plutonium, and video tapes that encode echocardiograms that were obtained from nonhuman primates with cardiac disease. The data base created by this activity has been used to develop interactive computerized models and algorithms (i.e., "smart systems") to facilitate diagnosis. Procedures to expand capabilities employed a video camera and off-the-shelf software to digitize serial radiographs obtained from dogs with progressive lung cancer. Continuing assessments are evaluating improved approaches that are enhancing the images and generating isometric (pseudo three-dimensional) colorized images and gray-scale enhancement techniques. 


\section{Quantification of Motility and Patterns}

Determinations of the frequency, magnitude, and other characteristics of movement have been employed in areas ranging from clinical human medicine through health evaluations of wild animal populations. This aspect of the project has been directed toward increasing the sophistication of current imaging methodologies and developing new integrated approaches to facilitate their use in the quantification of movement and movement patterns as measures of health or incipient disease at stages ranging from in utero life through the postnatal lifespan. The underlying hypothesis motivating this task is that the movement pattern of an organism is indicative of its state of health, and th $t$ deviations from normal characteristics can be used as an early sign of dysfunction.

Video tapes of human fetal ultrasonic examinations were made availajle to us which expedited preliminary efforts. Hardware and software currently supporting the Luboratory Directed Research and Development (LRDR) project, "Video-Based Imaging Interferometry," were adapted to analyze, selected sequences from these examination recorus. Using commercial image processing software, time sequences of the video record were digitized frameby-frame and segmented. The change in the twodimensional position coordinates of an identifiable anatomic structure (the fetal hand in the initial sequences) were measured as a function of frame number.

A white paper was prepared to describe the video experiments, results, and identification of modifications needed in subsequent experiments. Although restricted by video-frame rate, analyses of these data yielded several measures such as velocity, acceleration, direction, and position, as well as approaches to project movement into three dimersions. This study demonstrated the feasibility and capability of developing quantitative characterizations from clinical diagnostic imaging and identified shortcomings, such as sampling rate, that required addressing.

A second experim $-t$ was performed to assess the utility of data fron. normal and abnormal motion image sequences, both of which were acquired under comparable conditions. Because it was desired to obviate the influence of image acquisition characteristics in this experiment, tropical fish were selected as subjects for imaging using a standard video camera.
The fish were placed in a restricted volume and sequences of images for analysis were acquired under unperturbed conditions and after sedation. From these, the motion was characterized in these sequences by measuring spatial displacements, velocities, and accelerations as a function of time.

A white paper was prepared to describe the video experiments, results, and identification of modifications needed in subsequent experiments. Although only one level of sedation has been evaluated, it is noted that a characteristic metric should be defined so that the degradation of motility can be shown graphically as a plot of motility versus degree of sedation. This will demonstrate the potential for quantifying impairment of movement for neurotoxicity and neuropathophysiology. These capabilities will provide a basis to secure the necessary clinical collaborations and to seek external funding.

\section{Chemical Model of Cholelithiasis (Gallstone Formation)}

The hypothesis has been advanced that gallstone formation is increased by some of the dietary modifications that are introduced to avoid or treat other diseases. This aspect of the project adds an additional facet to existing programs to provide a basis for testing this hypothesis and exploring its ramifications. This in vitro chemical science extension of existing mammalian models is being used to predict the extent and time course of precipitation from bile. This approach involves the use of a thermodynamicsbased speciation-solubility model to determine the extent of oversaturation required to obtain nucleation and crystal growth.

The model was developed and refined through an in-depth, critical analysis of the literature that identified components of bile that have high probabilities of being important indicators or factors in stone formation. This information provided a foundation to rank the relative importance of individual components on stone initiation and growth. Collaborative evaluations of related literature are attempting to predict, insofar as possible, the effect of disease, diet, and other physiological conditions on the production and concentrations of important components.

An iterative series of efforts have been initiated to test the $\mathrm{r}$ rotypic predictive model relative to empirical data obtained from analysis of bile samples from the Pacific Northwest Laboratory (PNL) animal model. 
These comparisons are relating the probability of stone formation with individual bile components.

Sequential studies will test the hypotheses by making dietary changes to allow for iterative enhancements for refining the stone formation model. The model is serving as a basis to secure extramural funding for collaborative efforts. 


\section{Advanced Methods and Analytical Technology}

S. D. Harvey and J. A. Campbell (Chemical Sciences)

The objective of this project was to develop and expand PNL's capabilities in advanced methods of chemical and radiation measurement and environmental characterization technology. The goals were to produce a new resear ch capability for simultaneously determining the organic and inorganic constituents in metal complexes and other complex environmental samples, design advanced radiation detection instrumentation based on scintillation materials developed at PNL, and develop new methods for constructing detector systems.

\section{Separations}

There have been no literature reports describing electroseparation of aminopoly-carboxylic acid metal chelates. The unique nature of the separations developed for this program must be emphasized. A mixture containing the copper (II) complexes of EDTA, HEDTA, NTA, DTPA, and ED3A has been utilized for developmental studies. ED3A was synthesized and purified especially for these studies. Several different separation mechanisms have been investigated including both free zone elecirophoresis and micellar electrokinetic capillary chromatography. Capillary zone electrophoresis separation of the model copper complexes proceeded in a buffer consisting of $10 \mathrm{mM} \mathrm{Cu}(\mathrm{OAc})_{2}$ and $70 \mathrm{mM}$ acetic acid at $\mathrm{pH}$ of 5.5. This relatively simple buffer system provided resolution of the model compounds in under 45 minutes. The other systems developed for separation of the copper complexes involved buffers that incorporated surfactants at concentrations above their critical micellar concentration. One system utilized a buffer that contained $10 \mathrm{mM} \mathrm{Cu}(\mathrm{OAc})_{2}$ and $70 \mathrm{mM}$ acetic acid adjusted to a $\mathrm{pH}$ level of 5.5 that also contained $100 \mathrm{mM}$ cetyltrimethylammonium bromide. This system offers an extremely fast separation of the model compounds with a very high separation efficiency. As a direct consequence of the narrow band zones observed with this system, low detection limits were obtained. For example, a detection limit of approximately $40 \mathrm{pg}$ for DTPA was experimentally determined utilizing ultraviolet absorption at $290 \mathrm{~nm}$.
Another micellar system contained $5 \mathrm{mM} \mathrm{Cu}(\mathrm{OAc})_{2}$ with $35 \mathrm{mM}$ acetic acid at $\mathrm{pH}$ of 5.5 containing $75 \mathrm{mM}$ sodium dodecyl sulfate. The separation mechanism, in this case, was tased on Stern layer interaction between the surfactant and the negatively charged metal chelates through bridging copper cations. Although separations based on buffers that contain surfactants may be more difficult to interiace with both mass spectrometry and inductively coupled plasma, it must be acknowledged that these systems offer unique separation selectivity and can be used in conjunction with selective and ultrasensitive detection techniques such as miniaturized amperometric and radioisotope detectors.

The final separation that has been developed to date involves the separation of mixed metal EDTA complexes. A variety of metal chelates, including the EDTA complexes of $\mathrm{Co}(\mathrm{II}), \mathrm{Co}$ (III), $\mathrm{Cu}$ (II), Ni(II), $\mathrm{Fe}(\mathrm{III}), \mathrm{Y}(\mathrm{III})$, and $\mathrm{Cr}$ (III), have been prepared for these studies. In a preliminary separation, resolution between the Fe(III), Co(III), and Cr(III) EDTA complexes was obtained. Separation of these metal complexes was readily achieved with a $70 \mathrm{mM}$ acetate buffer at $\mathrm{pH}$ of 5.5 that contained $100 \mathrm{mM}$ dodecyltrimethylammonium bromide surfactant. Problems were encountered in this system when Co(II)EDTA was analyzed. It appears that electroseparation conditions promote decomposition of this metal chelate, perhaps by promoting oxidation of the metal center. It is therefore desirable to probe chromatographic-based separation mechanisms to avoid artificticious alteration of the metal oxidation states. Buffer systems that do not contain surfactant are presently being investigated.

\section{Mass Spectrometry}

Many of the chelators (free acid form, noncomplexed) have been analyzed using thermospray liquid chromatography/mass spectrometry. For example, both positive and negative ion ionization in the filament on and off modes were utilized to examine small organic acids including oxalic, formic, acetic, glycolic, glyoxylic, and citric acids. All of the acids except oxalic acid responded in positive ion thermospray with the filament on or off. Typical ions observed include $(\mathrm{M}+\mathrm{H})^{+}$and $\left(\mathrm{M}+\mathrm{H}_{2} \mathrm{O}\right)^{+}$. 
Simulated waste and actual waste samples were analyzed by thermospray liquid chromatography/mass spectrometry. Oxalic acid has also been analyzed using plasmaspray liquid chromatography/mass spectrometry; plasmaspray is similar to thermospray except a corona discharge is present to assist ionization. Spectra obtained using negative ion plasmaspray indicate $(\mathrm{M}-\mathrm{H})^{-}$as the base peak. In an important extension of this work, thermospray liquid chromatography/mass spectrometry was utilized to examine intact copper chelates. The liquid chromatography method was based on $\mathrm{Cu}$ complexation reversed-phase ion-pair chromatography that had been developed by W.R. Grace and further refined by our staff. Using this technique, we have examined several $\mathrm{Cu}$-complexed chelators by thermospray liquid chromatography/mass spectrometry. We have been able to separate $\mathrm{Cu}$ (EDTA) and Cu(HEDTA) and obtain mass spectra using thermospray liquid chromatography/mass spectrometry. The role of the metal is unclear at this point. Copper has an isotope pattern similar to that of $\mathrm{Cl}$. The $\mathrm{M}+2$ ion is approximately $33 \%$ of the intensity of $\mathrm{M}$. However, there is no evidence of $\mathrm{Cu}$ in the mass spectrum.
Electrospray mass spectrometry is another iowization technique that has shown promise for noncomplexed chelators that may be applicable for the intact metal complexes. Several of the chelators (free acids) have previously been analyzed using negative ion electrospray mass spectrometry and exhibit a base peak of (M-H)'. It is possible that this ionization technique will provide unique information for the intact metal chelates.

\section{Publications}

Campbell, J. A. "Mass Spectrometry of Complexed Metal Chelates." Pacific Northwest Laboratory, Richland, Washington, in preparation.

Harvey, S. D. "Capillary Separation of Aminopolycarboxylic Acid Metal Chelates." Pacific Northwest Laboratory, Richland, Washington, in preparation.

\section{Presentations}

Harvey, S. D., and J. A. Campbell. 1993. "Capillaryelectroseparation of Aminopolycarboxylic Acid Metal Chelates." Presented at the Actinides Separation Conference, Pasco Red Lion, Pasco, Washington (invited). 


\title{
Advanced Separations and Mass Spectrometry
}

\author{
R. D. Smith (Macromolecular Structure and Dynamics)
}

\begin{abstract}
Environmental cleanup u-der way at Hanford demands substantial advances in methodology and instrumentation for the analysis and characterization of environmental and biochemical materials. These advances are vitally dependent on the understanding of the basic chemical and physical phenomena underlying them. We addressed these needs in specific research activities that involve the development of tandem mass spectrometry, time-of-flight mass spectrometry, and Fourier transform ion cyclotron resonance mass spectrometry. Coupling these mass spectrometry advances with new high-resolution separation methods and better ion transport in magnetic fields and moderate pressures will provide for better and more sensitive methods of analysis and characterization that are important to the Hanford cleanup effort.
\end{abstract}

\section{Fourier Transform Ion Cyclotron Resonance Instrumentation}

A major emphasis of this research during FY 1993 was the development of mass spectrometry based upon electrospray ionization (ESI) combined with Fourier transform ion cyclotron resonance (FTICR) mass analysis. Improvements in the ion source design and FTICR operation have allowed us to demonstrate detection limits for small proteins of less than $10 \mathrm{fem}$ tomoles. The high pumping speed afforded by the instrumentation has allowed very high efficiencies for trapping of ions, as well as the ability to "cool" trapped ion populations. This has allowed higher resolution measurements to be obtained. Resolution in excess of $2.5 \times 10^{6}$ have been obtained for small proteins after correction for frequency shifts introduced by the nonideal electric fields inherent in current FTICR cell designs. The resolution measurements are $\sim 10^{3}$ better than obtained with conventional mass spectrometers, providing a basis for characterization of much more difficult materials and mixtures. We believe high-resolution measurements become less successful as molecular weight increases, due primarily to less effective "cooling" of the initial ion population. In order to address this limitation the capability for "quadrupole cooling," originally developed by physicists working with "Penning" traps, has been adapted to our instrumentation. By using quadrupole cooling we have also been able to demonstrate the ability to trap ions selectively in desired $m / z$ (mass to charge ratio) regions, allowing the cell to be filled with these ions and increasing the dynamic range of the FTICR measurements. We also have demonstrated that the on-line combination with both liquid chromatography and capillary electrophoresis was feasible, and that measurements using only low femtomole quantities of proteins can afford both high resolution and accurate mass measurements. Proteins of up to $30 \mathrm{~K} r$ a were measured at high resolution $(164,000)$.

Studies of Noncovalent Associations of Biopolymers by Mass Spectrometry

A number of important and highly specific noncovalent associations were successfully probed. These included oligonucleotide duplex and quadruplex structures, ribonuclease $S$ (a polypeptide-protein complex), and concanavalin A, the first example of protein quaternary structure determined by mass spectrometry. These studies were followed by more recent efforts showing that the quaternary structure of hemoglobin and avidin could be maintained. We were subsequently able to observe the complex of the avidin tetramer with four biotin molecules. Finally, we have been able to make initial measurements for myoglobin using FTICR where the noncovalently associated heme has been observed with the intact protein-indicating that complexes are subject to study by high-resolution FTICR. This is significant since it will allow determination of small solution species that play a role in stabilization of such co.aplexes.

These studies have demonstrated that very weak noncovalent complexes can be preserved into the gas phase, but their study requires that this is done at high $\mathrm{m} / \mathrm{z}$ (beyond the range of most existing instrumentation). These observations have provided new insights into the electrospray ionization process and the importance of Coulombic forces for the observation of labile associations. 


\section{On-lint Capillary Electrophoresis-FTICR}

The firs: on-line capillary electrophoresis-FTICR combination was demonstrated. Protein separations were obtained and molecular weights determined with high accuracy and sensitivity. High-quality and highresolution mass spectra were obtained with capillary electrophoresis injections of only 6 femtomoles.

\section{Electrospray Ionization-Orthogonal Time of Flight Mass Spectrometer Development}

A design was completed for a novel electrospray ionization-orthogonal time of flight aimed at obtaining ultrahigh sensitivity, high $\mathrm{m} / \mathrm{z}$ range, and very high speed (> 1000 spectra/second) using a new "perfectron" design. Computer simulations indicate that the resolution obtained from this device should be $>2000$. It is expected that the ultrahigh sensitivity feasible with this instrumentation will provide a basis for the first mass spectrometric studies at the single biological cell. Fabrication of components began in FY 1993, and assembly and testing should be conducted and completed in mid-FY 1994.

\section{Publications}

Bruce, J. E., G. A. Anderson, S. A. Hofstadler, B. E. Winger, and R. D. Smith. 1993. "Time-Base Modulation for the Correction of Cyclotron Frequency Shifts Observed in Long Lived Transients From Fourier Transform Ion Cyclotron Resonance Mass Spectrometry of Electrosprayed Biúpolymers." Rapid Commun. Mass Spectrom. 7:700-703.

Goodlett, D. R., D. G. Camp, C. C. Hardin, M. Corregan, and R. D. Smith. 1993. "Direct Observation of a DNA Quadruplex by Electrospray Ionization-Mass Spectrometry." Biol. Mass Spectrom. 22:181-183.

Goodlett, D. R., R. R. Ogorzalek Loo, J. A. Loo, J. H. Wahl, H. R. Udseth, and R. D. Smith. "A Study of the Thermal Denaturation of Ribonuclease $S$ by Electrospray IonizationMass Spectrometry." J. Am. Chem. Soc. Mass Spectrom., submitted.

Goodlett, D. R., J. H. Wahl, H. R. Udseth, and R. D. Smith. 1993. "Reduced Elution Speed Detection for Capillary Electrophoresis-Mass Spectrometry." J. Microcolumn Sep. 5:57-62.

Hofstadler, S. A., J. H. Wahl, J. E. Bruce, and R. D. Smith. 1993. "On-Line Capillary Electrophoresis with Fourier Transform Ion Cyclotron Resonunce Mass Spectromety." J. Am. Chem. Soc. 115:6983-6984.
Light-Wahl, K. J., J. A. Loo, C. G. Edmonds, R. D. Smith, H. E. Witkowska, C.H.L. Shackleton, and C.-S.C. Wu. 1993. "Collisionally Activated Dissociation and Tandem Mass Spectn metry of Intact Hemoglobin $\beta$-Chain Variant Proteins with Electrospray Ionization." Biol. Mass Spectrom. 22:112-120.

Light-Wahl, K. J., D. L. Springer, B. E. Winger, C. G. Edmonds, D. G. Camp, B. D. Thrall, and R. D. Smith. 1993. "Observation of a Small Oligonucleotide Duplex by Electrospray Ionization-Mass Spectrometry." J. Am. Chem. Soc. $115: 803-804$.

Light-Wahl, K. J., B. E. Winger, R. D. Smith. 1993. "Observation of the Multimeric Forms of Concanavalin A by Electrospray Ionization Mass Spectrometry." J. Am. Chem. Soc. 115:5869-5870.

Loo, J. A., C. G. Edmonds, and R. D. Smith. 1993. "Tandem Mass Spectrometry of Very Large Molecules. II. Dissociation of Multiply Charged Proline-Containing Proteins from Electrospray Ionization." Anal. Chem. 65:425-438.

Loo, J. A., R. R. Ogorzalek Loo, D. R. Goodlet, R. D. Smith, A. F. Fuciarelli, D. L. Springer, B. D. Thrall, and C. G. Edmonds. 1993. "Elucidation of Covalent Modifications and Noncovalent Associations in Proteins by Electrospray Ionization Mass Spectrometry." Techniques in Protein Chemistry III, 23-31. Academic Press, San Diego, California.

Ogorzalek Loo, R. R., D. R. Goodlett, R. D. Smith, and J. A. Loo. 1993. "Observation of Noncovalent Ribonuclease S-Protein/S-Peptide Complex by Electrospray Ionization Mass Spectrometry." Am. Chem. Soc. 1151:4391-4392.

Smith, R. D., D. R. Goodlett, and J. H. Wahl. 1993. "Capillary Electrophoresis" - Mass Spectrometry," CRC Handbook of Capillary Electrophoresis: A Practical Approach, ed. J. P. Landers, CRC Press Inc., 177-198.

Smith, R. D., and K. J. Light-Wahl. 1993. "The Observation of Noncovalent Interactions in Solution by Electrospray Ionization-Mass Spectrometry: Promise, Pitfalls, and Prognosis." Bio. Mass Spectrom. 22:493-501.

Smith, R. D., K. J. Light-Wahl, B. E. Winger, and D. R. Goodlett. 1993. "Electrospray Ionization." In: Biological Mass Spectrometry. eds. T. Matsuo, Y. Seyama, R. Caprioli, and $M$. Gross, in press.

Smith, R. D., J. H. Wahl, D. R. Goodlett, and S. A. Hofstadler. 1993. "Capillary Electrophoresis-Mass Spectrometry." Anal. Chem. 65:574A-584A.

Smith, R. D., J. H. Wahl, K. J. Light-Wahl, and B. E. Winger. 1993. "New Developments in Microscale Separations and Mass Spectrometry for Biomonitoring: Capillary Electrophoresis and Electrospray Ionization-Mass Spectrometry." J. Taxicology Environ. Health 40:147-158. 
Wahl, J. H., D. R. Goodlett, H. R. Udseth, and R. D. Smith. 1993. "Use of Small-Diameter Capillaries for Increasing Peptide and Protein Detection Sensitivity in Capillary Electrophoresis-Mass Spectrometry." Electrophoresis 14:448457.

Winger, B. E., K. J. Light-Wahl, R. R. Ogorzalek Loo, H. R. Udseth, and R. D. mith. 1993. "The Observation and Implications of High $\mathrm{m} / \mathrm{z}$ Ions from Electrospray IonizationMass Spectrometry." J. Am. Soc. Mass Spectrom. 4:536-545.
Winger, B. E., S. A. Hofstadler, J. E. Bruce, H. R. Udseth, and R. D. Smith. 1993. "High Resolution Accurate Mass Measurements of Biomolecules using a Novel Electrospray Ionization Ion Cyclotron Resonance Mass Spectrometer." J. Am. Soc. Mass Spectrom. 4:566-577. 


\title{
An Object-Oriented Model for Greenhouse Gas Emissions
}

\author{
M. A. Wise and J. A. Fox (Technology Management Planning)
}

\begin{abstract}
A simple object-oriented model of the supply and demand for greenhouse gas-emitting fuels in an economic equilibrium framework was constructed to more naturally represent the framework of an equilibrium model. An effective graphical humancomputer interface was constructed for that model. Based on the insight gained from building this model, the investigators will write a paper discussing the applicability and appropriateness of using an object-oriented approach for developing these models and the effects of using a welldesigned human-computer interface.
\end{abstract}

In this section, we briefly summarize the model on which we performed our object-oriented experiment: the Land Use Allocation Model (LUAM). The Land Use Allocation Model is a model of domestic agriculture and energy markets. The full details of the model are not central to the discussion here, and the description provided should not be considered a definitive exposition since the Land Use Allocation Model is still in an evolutionary phase.

As a pilot test for using an object-oriented programming approach for developing an economic model, the investigators started with the Land Use Allocation Model, already under development at PNL. In keeping with the standard acronyms of the field, we have dubbed the object-oriented version of the model to be the Object-Oriented Programming Land Use Allocation Model (OOPLUAM). The current version of OOPLUAM includes producing sectors for all crops that use large amounts of land in the U.S. (corn, soybeans, wheat, hay, etc.), as well as trees for wood products and biomass energy. Land allocation is modeled separately within each of 10 farm production regions, and it runs from 1990 to 2030 in 5-year time steps. The producing sectors, which include food and biomass energy crops, produce their output using a combination of 24 factor inputs. Each producing sector is modeled as a production function that computes production and input factor demands based on output prices, input prices, and technological coefficients. Land is allocated among competing producing sectors based on their relative profits per unit of land; therefore, the name Land Use Allocation Model.

The first step in designing the object-oriented programming model was to analyze the real-world system for potential classes. In searching for classes for OOPLUAM, we looked for common functionalities and logical groupings of the economy such as producing sectors, factors of production, and regions. First, we divided the model into classes (or object definitions) and decided what information needs to be communicated via messages among objects and between objects and the main program. The implementation of functionalities within each object was considered separately. In other words, the objectoriented programming approach specifies that the interface of each object with the rest of the model be considered separately from the inner workings of each object. This process is a divide-and-conquer strategy that not only helps to manage complexity, but also results in program modularity.

The most pervasive class and object structure in OOPLUAM is that of a producing sector. A producing sector is an economic entity that transforms factor inputs like labor and capital into an output like corn or coal. Some data elements and methods are shared by most producing sectors, but some data elements and methods are specific to only a subset of the producing sector classes. In this situation, a class hierarchy with inheritance was used to exploit all the commonality while maintaining the capability of incorporating exceptions and additions on an asneeded basis.

The investigators concluded that the object-oriented programming approach to building an energyeconomic model offers significant advantages over the procedure-oriented approach we have been using. However, the gains from object-oriented programming may not be apparent until model changes become necessary. It could also be argued that $\mathrm{C}++$ is a more complicated language to master than most procedural languages such as FORTRAN and Pascal. However, when a library of reusable objects or 
classes exists that can applied to the new model, programming it may be much faster with an objectoriented programming language.

A more detailed summary of our conclusions is provided below.

Encapsulation, the process of binding code to data to create programming objects, is the furdamental advance of object-oriented programming over procedural programming. With encapsulation, an object has to be told only once, during its construction, what it is and what its procedures are. Because these procedures are bound to the object, an object remembers them throughout the course of a model run (unless, of course, it is designed as a temporary object with limited duration within the model).

Modularity is certainly possible using a conventional procedural language, but it is often a struggle to achieve and maintain. In contrast, modularity is the default result of an object-oriented programming language. Object-oriented programming lends itself more naturally to modular programming because the interface among objects is distinct from the implementation of the objects' internal procedures, the details of which are hidden from the rest of the model. This makes object-oriented programming an ideal approach for a multiprogrammer project. The modularity of object-oriented programming facilitates a division of labor, and it minimizes the need for the programmers to keep close communication about mundane program details. Object-oriented programming's modularity also makes it ideal for models that will undergo an evolutionary design, as most energyeconomic models must do in response to changing policy questions.

In contrast to subroutines, classes (and their objects) are self-contained programming entities. Therefore, class definitions from one program can be readily reused in other programs. For example, the producing sector classes described here for OOPLUAM could easily be used in a different economic model. Another model could use these classes to construct objects directly, or it could use any of these classes as inheritance bases for deriving new classes that meet some specialized need of a new model. Moreover, object-oriented programming makes it possible to construct a powerful class library of generic economic entities that could serve as the building blocks of several different models. An object-oriented programming class library is superior to a conventional subroutine library of functions because of the encapsulation of the data structure and all related functions into distinct objects.

\section{Publication}

Wise, M. A., J. Fox, and R. Sands. 1993. "An ObjectOriented Approach to Energy-Economic Modeling." In Energy \& the Environment. Proceedings of the Fiftcenth Annual North American Conference of the International Association for Energy Economics (IAEE), Seattle, Washington. 


\title{
Analysis of Non-Statutory Mechani-ims for Implementation of Global Environmental Policies: Methodology Development for Public Policy and Industrial Information Needs
}

\author{
A. M. Lesperance (Technology Policy Analysis)
}

Scientists predict that some regions of the globe will experience environmental changes in the upcoming decades. Policymakers are developing international agreements to mitigate the potential impact of this environmental change. These policies, once adopted and implemented, will have a direct impact on industry's decisions about what, how, and where to manufacture goods.

The objectives of this project were to provide insight inso the information needs of global climate change policymakers and the industrial community impacted by those policies (consumer/ public), and provide recommendations to the scientific community about possible gaps in the current knowledge base that have direct bearing on policy and industry decisions.

Possible environmental change and existing environmental problems increasingly spur the scientific community to provide research data to policymakers and industry in order to regulate the known or anticipated negative environmental impacts of society's actions. The policies and information are frequently intended to influence industry's decisions with regard to what, how, and where goods are manufactured.

However, policymakers and rescarchers know little about how decision makers in industry factor science and policy into their individual choices. Such insight would help to increase the practical value of environmental studies by focusing research on the information that industry needs and by communicating research results in ways that make the information accessible and applicable to decision makers.

This study explored ways in which information is used by industry to respond to environmental concerns and agreements. The objective is to describe, from industry's perspective, the decisions they face and the information they need to manage decisions with environmental impacts. The ultimate goal of this line of study is to establish a research agenda that will deliver useful data to decision makers and policymakers. A practical consequence of the research is to help focus national science and technology strategies to produce and transfer information to industry.

To effectively influence decisions, one must first understand what the decision situations are and how decision makers perceive those opportunities. To build a decision-focused environmental research agenda, the scientific community needs to know

- what decisions industry is currently conscious of making that impact the environment

- what information decision makers use to make their decisions and where do decision makers get their information

- what information does industry need, but is currently missing

- how information should be packaged (from whom should it come, in what detail) to make it useful.

A paper was developed that presented a case study of one segment of the wood products industry in the Northwest-mills or wood treating plants. The data came from interviews which focused on the questions stated above. The interviews were conducted with industry decision makers in small to medium-sized companies in Oregon and Washington and were conducted at their facilities.

\section{Strength of Relationship}

This study found the relationship between industry and science to be weak in terms of information needs and sources of information. Scientific information is being presented to industry from both the scientific community and from the regulatory conmunity. The information used for regulatory decisions comes from the scientific community by way of the regulatory 
community, whereas information that is not regulation driven, comes directly from the scientific community.

\section{Information Need and Flow}

During the interviews, we heard three issues concerning the information flow from the scientific community to the industrial community. First, there were some industry decision makers who felt that the information was not needed. Some felt that the information was adequate, but there was not a vehicle for the information to be passed to the industrial community, and lastly, there were some that felt that scientific information could be made more useful. There were several decision makers that did not feel they could use scientific information for decision making. They had enough information from the regulatory agencies to deal with environmental compliance. The industry decision makers would like to see the industry incorporated into models. They see the need for a more global view of science.

\section{Honest Broker - Who To Trust}

Industry does not have trust in the scientific community. They feel that the scientific community lacks objectivity and, therefore, can be hired to express a desired view. Because of this mistrust they are confused about who is the "honest broker." They felt that the results of research would reflect the viewpoint of the person who funded the research. When it came down to who do they trust, it was dependent on individuals in the scientific community. There was no trust in organizations (e.g., Forest Service, universities) as a whole, but rather, trust was based on individual relationships with scientists. Additionally, sometimes good science is produced, but not used. For example, a decision maker stated that the Forest Service provides data and good science, but the problem is what is done with the information. There needs to be a way in which to find and use this information.

\section{Broader Scope Needed}

Scientists need to have a big-picture attitude, and to understand industry perceptions and the types of decisions they make. The scientific community needs to improve their ability to transfer science from the laboratories into the field. There needs to be costbenefit analysis done to address the demand for wood products within the context of environmental concerns. 


\title{
Characterization of Structure and Dynamics of Surface Adsorbates and Their Surfaces
}

\author{
P. D. Ellis (Macromolecular Structure and Dynamics)
}

The objectives of this project included the following:

- perform solid-state nuclear magnetic resonance (NMR) spectroscopy of four-surface systems

- characterize the surface molybdenum sites on hydrodesulfurization (HDS) catalysts

- address the specific details associated with the characterization of quadrupolar nuclides via a general line shape procedure.

This project supported the research activities of Drs. P. D. Ellis, M. A. Kennedy, and M. K. Bowman, all of whom were involved in the development of the nuclear magnetic resonance spectroscopy capabilities for the Molecular Science Research Center/ Environmental and Molecular Sciences Laboratory.

Research undertaken by Dr. Ellis' graduate students from the University of South Carolina resulted in a protocol for use in optimizing the surface loading (i.e., maximizing the nuclear magnetic resonance signal) for a given loading of the alumina surface with silver.

Research also resulted in a solution to a persistent problem related to the fitting of line shapes. The line shapes of interest in this area generally arise from the presence of two tensors denoted as $\mathbf{A}$ and $\mathbf{B}$. To add tensors, one tensor must be expressed in the coordinate system of the other. In practice, the Euler angles, $\underline{a}, \underline{b}$, and $\underline{c}$, are usually used to express the tensors in compatible terms that can be added.

Standard multiple-parameter nonlinear least squares methods normally can be used to fit the line shapes of interest in this research; however, that approach results in a high probability that one of the tensors will have a sign convention violated with regard to the definition of tensor A (for example). This problem manifests itself as an apparent change in the handedness of the coordinate system describing tensor $A$ (i.e., it would become a left-handed system while tensor B would remain a right-handed system).

The problem was solved when it was realized that its origin resulted from the fact that the Euler angles were simply crossing some well-defined points; that is, it can be demonstrated that $90^{\circ}$ increments of the angle $\underline{c}$ can lead exactly to the observations made in our research. Likewise, incrementing angle a by $90^{\circ}$ would have the same impact on tensor $B$. This finding has been included in our line-fitting program, thus eliminating the problem. Our line-fitting program now converges in half the number of steps than previously needed and with a lower sum of residuals.

In other progress, the nuclear magnetic resonance spectrometers that are key to achieving the experimental objectives described above are scheduled to be installed in early FY 1994.

Publication

A paper describing line-shape theory is in the final stages of preparation. 


\title{
Chemical Adducts and Radiation Sensitivity of Supercoiled DNA
}

\author{
J. M. Nelson (Biology and Chemistry)
}

\begin{abstract}
Normal biological processes of transcription, replication, and gene recombination introduce varying degrees of deoxyribonucleic acid (DNA) supercoiling. In studies with DNA plasmids, it has been found that supercoiling enhances the sensitivity of DNA to strand breakage by ionizing radiation. This project addressed the effect of additional perturbations to the DNA structure introduced by aberrant structures, including base mismatches, hairpin- or cruciform-forming structures, and chemical adducts on radiation damage to supercoiled DNA. DNA plasmids were constructed that contained structural anomalies at specific locations and had specific degrees of superhelicity. These plasmid constructs enabled mechanistic studies of the effects of perturbed DNA structures on the radiation sensitivity of physiologically stressed DNA.
\end{abstract}

Miller et al. (1991) found that negative supercoiling increased the sensitivity of plasmid DNA to singlestrand break induction by $x$-rays. This earlier work was done with the PIBI-30 plasmid in TE buffer (10 mM Tris, 1 mM EDTA; pH 7.2) as the target molecule and the DNA was irradiated at $2^{\circ} \mathrm{C}$. They quantified the levels of Form I, Form II, and Form III plasmid using densitometry of ethidium bromide stained gels following ultraviolet treatment. This treatment with ultraviolet light fragments the DNA on the gel permitting equivalent ethidium bromide binding regardless of which form the DNA molecule existed prior to fragmentation or ultraviolet nicking. Milligan et al. (1992) re-examined the Miller et al. study but found no change in the $x$-ray sensitivity of the plasmid as a function of superhelicity. Their studies used the pUC-18 plasmid in tris buffer, and they irradiated it at $2^{\circ} \mathrm{C}$. They also quantified the plasmid DNA levels using densitometry of ethidiumbromide stained gels after determination of the binding constants of ethidium bromide to covalently closed circular pUC 18 at several levels of superhelicity. Swenberg et al. (1990) repeated the work of Miller using pIBI 30 at room temperature using $\gamma$-rays and found that the radiation sensitivity of the first strand break increased only when the negative superhelicity was absolutely greater than $\Delta \mathrm{L}_{\mathrm{k}}=-2.5$. However, they also found that the pIBI-30 plasmid demonstrated its lowest sensitivity at negative superhelical densities around $\Delta \mathrm{L}_{\mathrm{k}}-2.5$ with the sensitivity increasing again as the DNA becomes less negatively supercoiled. The plasmid was irradiated in a low-scavenging capacity phosphate buffer ( $\mathrm{pH} 7.2$ ); they used the method of Milligan et al. (1992) to determine the concentration of the various forms of plasmid. Clearly, there is some controversy about the observations of Miller et al. (1991).

In the LDRD proposal submitted last year, we suggested synthesizing DNA inserts for plasmids that might demonstrate enhanced $x$-ray sensitivity. However, before we could spend much time and effort in the preparation of these inserts, we had to verify that the phenomena reported by Miller et al. (1991) and Swenberg et al. (1990) was indeed real.

\section{Technical Progress}

We decided to use an alternative method for determining the induction of single-strand breaks in DNA in order to assess the effect of $x$-irradiation on supercoiled DNA. As considerable effort was required to adapt this alternative method for quantification of DNA breaks, no experimental effort was made to investigate induction of structural perturbations resulting from aberrant DNA structures such as base mismatches, hairpin- and cruciform-forming structures, or chemical adducts, as originally intended. Similarly, we were unable to begin construction of plasmids containing these structural anomalies, or having specific degrees of negative or positive superhelicity.

To investigate the sequence specificity and superhelical specificity of $x$-ray induced DNA damage we wanted to examine the issue again using a completely different technique which would avoid the use of 
ethidium bromide. We selected Southern-blot analysis using radiolabeled probes as a suitable alternative technique.

First, we developed an unambiguous method of quantifying plasmid DNA Forms I and II (the form arising from a radiation-induced single-strand nick). We selected Southern-blot techniques, radiolabeled plasmid probes, and densitometric determination of the probed undamaged and damaged plasmid species as the method of choice. Although technically much more demanding than the ethidium bromide technique that is in common use, most consider it superior. During this past year we developed the technique, calibrated it, and showed that we could generate good standard curves of DNA concentration versus Southern blot band intensity. We perfected the technique and wrote a report.

Second, we spent the greater part of the year determining the $x$-ray sensitivity of plasmid DNA as a function of superhelicity, ionic strength, and temperature. We selected the plasmid pBR 322 as our target because of its commercial availability and desirability as a target with only one or at most a few supercoiling-induced single-strand regions. We prepared pBR 322 at six topoisomeric conformations $\left(\Delta \mathrm{L}_{\mathrm{k}}=-4,-9,-14,-19,-22\right.$, and -26 native or wildtype plasmid) and in two phosphate buffer concentrations (10 mM and $50 \mathrm{mM})$. Each of the topoisomers of plasmid DNA was irradiated (in duplicate) at five temperatures $\left(10,20,30,40\right.$, and $\left.50^{\circ} \mathrm{C}\right)$. We have completed the analysis of about two-thirds of these irradiations; enough to see that there is a lower sensitivity of pBR-322 plasmid DNA at $\Delta \mathrm{L}_{\mathrm{k}}=-4$ and -9 compared to the higher superhelical densities.

However, these data are preliminary and we still have some irradiations to do at the low superhelical density to verify our results.

Third, we intended to develop a piece of sensitive DNA and determine if insertion of this piece into a plasmid would lead to increased radiation sensitivity. We expect to begin work on this project when Dr. Fleck returns to PNL next summer.

\section{References}

Miller, J. H., J. M. Nelson, M. Ye, C. E. Swenberg, J. M. Speicher, and C. J. Benham. 1991. "Negative Supercoiling Increases the Sensitivity of Plasmid DNA to Singlo-strand Break Induction by X-ray." Int. J. Radiat. Biol. 59(4): 941-949.

Milligan, J. R., A. D. Arnold, and J. F. Ward. 1992. "The Effect of Superhelical Density on the Yield of Single-strand Breaks in $\gamma$-Irradiated Plasmid DNA." Radiat. Res. 132:69-73.

Swenberg, C. D., E. A. Holwitt, and J. M. Speicher. 1990. "Superhelicity and DNA Radiation Sensitivity." In SAE Technical Paper Series \#901349, pp. 1-7, Society of Automotive Engineers, Inc., Warrendale, Pennsylvania. 


\title{
Chemical Desorption/Dissolution Kinetics
}

\author{
C. C. Ainsworth (Geosciences)
}

\begin{abstract}
Desorption of metals and radionuclides from important mineral phases associated with subsurface materials was probed with new methods to provide a basis for expanded research on the fundamental chemical mechanisms governing desorption. Pressure jump kinetics were introduced (to PNL) through new university relationships, and electron paramignetic resonance spectroscopy capabilities were extended to quantitate the effects of master variables ( $p H$, solution composition, ionic strength) on the rate limiting steps in desorption and linear free energy relationships between metals and several mineral phases. The results provided new insights into the use of advanced chemical approaches for resolution of the mechanisms of contaminant behavior in subsurface environments.
\end{abstract}

Developing an understandin ${ }_{6}$ of the kinetic processes governing the distribution of metals and radionuclides in solid surface-aqueous systems, typical of the complex subsurface environments, requires resolution of difficult technical obstacles. The present studies utilized two new techniques (stop-flow and pressurejump) to address several of these obstacles. The results of these studies have extended our knowledge of the adsorption/desorption process at the surface of environmentally important minerals for a number of cationic and anionic metal species.

In FY 1991, a stop-flow system was combined with an electron paramagnetic resonance spectrometer to measure reactant species in situ on millisecond time scales in aqueous suspensions. This technique was utilized to determine the rates of adsorption and desorption of $\mathrm{Mn}^{2+}$ on the surface of Birnessite $\left(\mathrm{MnO}_{2}\right)$ as part of a study of $\mathrm{Cr}^{3+}$ oxidation at the surface of $\mathrm{MnO}_{2}$. The $\mathrm{Mn}^{2+}$ sorption reaction was complete within 1 second, with greater than $80 \%$ of the $\mathrm{Mn}^{2+}$ sorbed within $200 \mathrm{~ms}$. A first-order rate dependence was observed, and by using an integrated reversible first-order rate expression, the desorption reaction was determined. The $\mathrm{Mn}^{2+}$ desorption rate was calculated to be more than an order of magnitude smaller than the adsorption rate suggesting that the desorption process is considerably slower than the adsorption process. Several inorganic metals (Mn, $\mathrm{Cu}, \mathrm{V}$ ) and many organic free radicals and radical cations are electron paramagnetic resonance spectroscopy probes. Through the use of appropriate probes, the reaction kinetics of important geochemical reactions may be discerned at time scales that will allow a more complete understanding of mechanisms and pathways.

In late FY 1992 and FY 1993, investigations centered on the kinetics of $\mathrm{Cu}^{2+}, \mathrm{H}_{2} \mathrm{AsO}_{4}^{-}$, and $\mathrm{CrO}_{4}{ }^{2-}$ adsorption/desorption on goethite $(\alpha-\mathrm{FeOOH})$. These processes were evaluated using the pressure-jump relaxation technique with conductivity detection. This method allows mechanistic information to be obtained for the elementary processes occurring at millisecond time scales. It is based on the fact that chemical equilibria are dependent on pressure; a small pressure perturbation results in a shift in the chemical equilibria causing the system to shift (relax) to the new equilibria conditions. The rate of this relaxation is determined by the rate of the chemical reactions that make up the equilibrium state.

In the case of $\mathrm{Cu}^{2+}$ sorbed to the surface of goethite, a double relaxation event was observed. The rate of these relaxations ( $\tau$, tau) decreased with an increase in $\mathrm{pH}$. The fast relaxation was ascribed to a reaction mechanism associated with the formation of a monodentate inner-sphere $\mathrm{Cu}^{2+}$ /goethite surface complex; further, it was speculated that the two observed relaxations were the result of the same mechanism, but the difference between the two observed relaxations resulted from differences in the adsorption energy of different sorption sites. A modified triplelayer surface complexation model was used to describe the adsorption of $\mathrm{Cu}^{2+}$ on goethite and a pH-stat proton release study was conducted to confirm the mechanism proposed above. The intrinsic rate constant for adsorption $\left(k_{1}\right.$ int $)$ was about two orders of magnitude larger than the intrinsic rate constant for desorption. Using results from this study and others, it was established that the rate of adsorption of divalent metal cations on goethite was directly related to the rate of removal of a water molecule from the primary hydration sphere of the metal ion. 
Similar to $\mathrm{Cu}^{2+}$, single relaxations observed for $\mathrm{H}_{2} \mathrm{AsO}_{4}{ }^{-}$, and $\mathrm{CrO}_{4}{ }^{2-}$ sorbed to the surface of goethite were ascribed to a reaction mechanism associated with the formation of a monodentate inner-sphere $\mathrm{HAsO}_{4}{ }^{-}$, and $\mathrm{CrO}_{4}{ }^{2-} /$ goethite surface complex. The $\mathrm{CrO}_{4}$ surface species, in the past, have been described as an outer-sphere surface complex. However, spectroscopic interrogation of the surface-sorbed $\mathrm{HAsO}_{4}$, and $\mathrm{CrO}_{4}{ }^{2-}$ by $\mathrm{x}$-ray absorption spectroscopy tends to confirm the inner-sphere surface complex. In both of these cases, adsorption is markedly faster than desorption. For example, the rate constant for $\mathrm{HAsO}_{4}$ adsorption is approximately four orders of magnitude larger than the $\mathrm{HAsO}_{4}$ desorption rate constant.

\section{Publications}

Fendorf, S. E., D. L. Sparks, J. A. Franz, and D. M. Camuioni. 1993. "Electron Para-Magnetic Resonance Stopped-Flow Kinetic Study of Manganese(II) SorptionDesorption on Birnessite." Soil Sci. Soc. Am. J. 57:57-62.

Grossl, P. R., D. L. Sparks, and C. C. Ainsworth. "Rapid Kinetics of Cu(II) Adsorption-Desorption on Goethite $(\alpha-\mathrm{FeOOH})$." Environ. Sci. Technol., in press. 


\title{
Chemical Sensors
}

\author{
J. W. Grate (Materials and Interfaces)
}

The objective of this project was to develop a new capability to examine, at the microscopic level, the interaction between environmental pollutants and chemically selective sensor materials, the kinetics and thermodynamics of processes occurring at these interfaces, and to develop the understanding required jor the rational chemical and physical modification of materials for integration into chemical sensor devices. Additional work was directed at the development of advanced sensor calibration techniques that will ultimately enhance the sensitivity and selectivity of sensors and arrays of sensors.

Surface acoustic wave (SAW) sensors are being examined for their usefulness in environmental applications. These sensors utilize a sorbent polymer layer to collect and concentrate organic vapors from the atmosphere. Models for vapor sorption by particular polymers have been compiled and are being used to estimate polymer/gas partition coefficients and sensor responses for hundreds of vapors. From these, it can be determined if a polymer-coated sensor is likely to be able to detect particular organic vapors of environmental interest with sufficient sensitivity. This provides a streamlined, rational approach to sensor design, and provides guidance for the development of new chemically selective sorbent materials.

Arrays of sensors are used to improve selectivity. Instrumentation to facilitate sensor array system research and prototype testing is being developed. It incorporates surface acoustic wave resonators with exceptional stability in an array system of six surface acoustic wave sensors, with sensor temperature control and a microcontroller to count the sensor frequencies and report them on a serial line. The controller will also be capable of automating pumps and valves in a sampling system as prototypes are developed. In addition, these systems provide the instrumental basis needed to pursue basic research in selective materials and interactions for surface acoustic wave sensors.
New designs of silicon-based CHEMFET (field effect transistor chemical sensor) sensors been fabricated and characterized. Experiments were conducted to map the migrating surface ions in lateral electric fields, contributing to a better understanding of the baseline drift of FET-based gas sensors. This work also has fundamental applications for studying the charge distribution on insulator surface with scanning probes or when measuring local capacitance. Suspended gates with temperature control have been fabricated, and the temperature characteristics of the gates are being characterized. These are critical for the development of CHEMFET-basid systems for hydrogen detection. The systems planned will incorporate arrays of CHEMFET sensors, varying the selective thin films applied to the gate structure, and the thin film temperature.

Novel polymer/metal cluster composite materials have been prepared by electrochemical techniques. These thin films promise to open a new frontier in chemically selective thin films, where the selectivity can be tailored by tailoring the choice of metal, cluster size, and polymer matrix. A CHEMFET sensor utilizing a polyaniline/ $\mathbf{H g}$ composite layer was developed for detection of small amounts of $\mathrm{HCN}$ in air.

Pattern recognition analysis and chemometrics are essential for analyzing the data from higher order microanalytical systems, such as those employing the surface acoustic wave and CHEMFET sensors described above. Fundamental studies in first order and second order chemometric methods have been conducted.

A major review of chemical sensing needs in environmental restoration and protection has been prepared. Parts of this review will help to define environmental sensing needs at Hanford. In another review the new class of chemically selective sensing layers, based on electroactive polymers, has been discussed.

Flow injection analysis is a microanalytical methodology for analysis on small liquid samples. A flexible 
state-of-the-art flow injection analysis system, with associated hardware and computer control, is being used to develop new microanalytical techniques. Experiments have been conducted in contacting and then separating water and transuranic extraction (TRUEX) solvents. This work is intended to establish a methodology for liquid-liquid extraction schemes in the analysis and processing of tank wastes. This methodology increases the automation and decreases the labor in performing these extractions in the laboratory, as well as reducing waste. A number of other potential applications for flow injection analysis have been identified, including $\mathrm{Cr}(\mathrm{VI})$ analysis, soil buffer capacity analysis, use as a sample input method for inductively coupled plasma-mass spectrometry, and detection of radionuclides. Demonstration of principle for these applications is planned. Initial experiments to design and calibrate a system for $\mathrm{Cr}(\mathrm{VI})$ analysis have already been completed. It is significant that flow injection analysis systems can be made to be field portable. To our knowledge, our flow injection analysis capability is rare (if not unique) in the national laboratory system.

Efficient chemical sensor development requires the ability to gen. ste test samples. The chemical sensors program is establishing capabilities to test gas phase sensors against a wide range of gases and vapors, including complex mixtures. An organic vapor generation system is being automated so that it can create and dilute a gas stream containing one of eight user-selectable vapors and perform preprogrammed sequences of experiments. A humidity generating system that delivers humidified air at user-set flow rates and relative humidities will be used to generate background humidities to simulate real-world conditions. Mass flow control systems are being used to set up a gas handling system for compressed gas mixtures. These development activities, with permeation tube ovens purchased in the previous year, create a comprehensive capability in creating test samples for gas sensor testing.

The ability to deposit adherent thin films is essential to chemical sensor development and their long-term performance. In addition to conventional methods such as spin coating and deposition from an aerosol, electrochemical methods are being used extensively, and a Langmuir Blodgett trough has been set up for preparing thin films of amphiphilic molecules and polymers. A unique scanning tunneling and atomic force microscope system has also been developed for studying the structure of adsorbates at the liquid/vapor and liquid/air interfaces.

\section{Publications}

Rruckner-Lea, C., J. Janata, J. Conroy, A. Pungor, and K. aldwell. "Scanning Tunneling Microscopy on a Mercury Sessile Drop." Langmuir, in press.

Domansky, K., J. Janata, M. Josowicz, and D. Petelenz. 1993. "Present State of Fabrication of Chemically Sensitive Field Effect Transistors." Analyst 118:335.

Domansky, K., Y. Leng, C. C. Williams, J. Janata, and D. Petelenz. "Mapping of Mobile Charges on Insulator Surfaces with the Electrostatic Force Microscope." Applied Physics Letters, in press.

Domansky, K., D. Petelenz, and J. Janata. "Lift-off Process for Noble Metals." Journal of Vacuum Science and Technology $B$, in press.

Grate, J. W. "Sensing Glass Transitions in Thin Polymer Films on Acoustic Wave Microsensors." ASTM Special Technical Publication on the Assignment of the Glass Transition, in press.

Grate, J. W., S. J. Martin, and R. M. White. 1993. "Acoustic Wave Microsensors, Part I." Anal. Chem. 65:940A-948A.

Grate, J. W., S. J. Martin, and R. M. White. 1993. "Acoustic Wave Microsensors, Part II." Anal. Chem. 65:987A-996A.

Grate, J. W., S. L. Rose-Pehrseon, M. Klusty, and H. Wohltien. 1993. "A Smart Sensor System for Trace Organic Vapor Detection Using a Temperature-controlled Array of Surface Acoustic Wave Vapor Sensors, Automated Preconcentrator Tubes, and Pattern Recognition." In: Proceedings of the Symposium on Chemical Sensors II, Volume 93-7, The Electrochemical Society, Inc., New Jersey, pp. 597-608.

Janata, J., and B. M. Wise. Environmental Sensors, Encyclopedia of Energy Technology and the Environment. J. Wiley \& Sons, Inc., New York, in press.

Josowicz, M., and J. Janata. 1993. "Electroactive Polymers in Chemical Sensors." In Electroactive Polymers. ed. B. Scrosati, p. 310.

Li, J., D. Petelenz, and J. Janata. 1993. "Suspended Gate Field-Effect Transistor Sensitive to Gaseous Hydrogen Cyanide." J. Electroanalysis 5:791-794.

Tian-Hong, Z., D. Petelenz, and J. Janata. 1993. "Temperature-controlled Kelvin Microprobe." Sensors and Actuators 12:175-180. 
Wise, B. M., and N. L. Ricker. 1993. "Identification of Finite Impulse Response Models with Continuum Regression." J. Chemometrics 7:1-14.

\section{Presentations}

Domansky, K. 1993. "Suspended Gate Field Effect Transistor." Presented at the PNL/Sandia/WHC Microsymposium on Solid-State Hydrogen Sensing Devices, Requirements and Strategies, Richland, Washington.

Grate, J. W. 1993. "Polymer Coatings for Acoustic Wave Vapor Sensors and Arrays." Presented at the JASON 1993 Summer Study, GA Technologies, San Diego, California (invited).

Grate, J. W. 1993. "Sensing Glass Transitions in Thin Polymer Films on Acoustic Wave Microsensors." Presented at the ASTM Special Technical Symposium on the Assignment of the Glass Transition, Atlanta, Georgia (invited).

Grate, J. W. 1993. "Solvent Effects on the Decarboxylation of 3-Carboxybenzisoxazole Ions: The Predominant Role of Hydrogen Bonding." Presented at the Meeting on Solute/ Solvent Interactions, Aberdeen Proving Ground (invited).

Grate, J. W., R. A. McGill, and M. H. Abraham. 1993. "Chemically Selective Polymer Contings for Acoustic Vapor Sensors and Arrays." Presented at the 1992 IEEE Ultrasonics Symposium, Tuscon, Arizons (invited).

Grate, J. W., S. L. Rose-Pehrsson, M. Klusty, and H. Wohltjen. 1993. "A Smart Sensor Systems for Trace Organic Vapor Detection Using a Temperature-controlled Array of Surface Acoustic Wave Vapor Sensors, Automated Preconcentrator Tubes, and Pattern Recognition." Presented at the 183rd Meeting of the Blectrochemical Society, Honolulu, Hawaii (invited).
Janata, J. 1993. "Chemical Modulation of Work Function in Chemical Sensors." Presented at the University of Oregon, Eugene (invited).

Janata, J. 1993. Chemical Sensors, eight lectures in an Intensive Sensor Course, Kreuth, Germany (invited).

Janata, J. 1993. "Environmental Sensing at Hanford." Presented at the University of Illinois, Urbana (invited).

Janata, J. 1993. "Environmental Sensing at Hanford." Presented at the University of Oregon, Eugene (invited).

Janata, J. 1993. "Environmental Sensing at Hanford." Presented at the University of Utah, Salt Lake City (invited).

Janata, J. 1993. "Environmental Sensing at Hanford." Presented at the University of Washington, Seattle (invited).

Janata, J. 1993. "Ion Optodes." Presented at the Electrochemical Society Meeting, Honolulu (invited).

Janata, J. 1993. "New Sensing Principles." Presented at the Pittsburgh Spectroscopic Society, Pittsburgh (invited).

Janata, J. 1993. "Sensor Technology." Presented at the ALCOA Technical Center, Pittsburgh (invited).

Janata, J. 1993. "Solid State Chemical Sensors." Presented at the Columbia Basin Technical Conference, Pasco, Washington (invited).

Janata, J., C. Bruckner-Lea, J. Conroy, A. Pungor, and K. Caldwell. 1993. "Scanning Tunneling Microscopy on a Mercury Sessile Drop." Presented at the 1993 ACS Meeting, Chicago Illinois (invited). 


\title{
Child Health and Welfare Index
}

\author{
J. D. Longstreth (Technology Planning and Analysis)
}

This project is a continuation of work started in FY 1992 on the Child Health and Welfare Index.

Efforts were continued to identify appropriate Second Generation Edmonds-Reilly Model (SGM) outputs with which to link index parameters. Empirical algorithms were developed to specify the relationship between the index elements and the Second Generation Edmonds-Reilly Model output. Changes in the index were ther examined under various scenarios of global change.

The Child Health and Welfare Index (CHWI) is being developed as a way to assess the health and welfare status of a nation in order to integrate this information into modeling efforts to assess the potential impacts of global climate change. Two versions have been developed: 1) World-CHWI and 2) U.S.-CHWI. Most of the work done this year has been on the latter version.

The World-CHWI incorporates data on female literacy, maternal mortality, infant mortality, 1 to 5 yearold mortality, low birth weight, primary education, daily protein available per capita, access to safe water, and access to health care. These data elements have been developed into an index that provides a ranking across 156 countries for a single point in time: 1991 . The intent of including these data in an index is to create a metric for the assessment of human health and welfare, which is based on the most susceptible human population-children. This sensitive subpopulation should act like a sentinel for the impacts of global environmental change on human health and welfare; if children are not affected, it is likely that the rest of the human populations will also remain unaffected. In the near-term the index is intended to provide policymakers and others concerned with public health policy issues a simple tool by which to gauge the health and welfare status of nations throughout the world. The long-term goal for this research is to link this index to the economic models being used to assess the potential consequences of global climate change.

The U.S.- CHWI evaluates the health and welfare status of U.S. populations temporally over the period between 1920 and 1990 . It incorporates all of the World-CHWI data elements except access to safe water and health care for which temporal data in the U.S. were unavailable. This version of the Child Health and Welfare Index was created in order to explore connections to the Second Generation Model. Although there are nine regional models programmed for the Second Generation Model, only the one for the U.S. is fully populated with data. In FY 1993, we successfully developed the U.S.-CHWI and began to explore the appropriate connection to the Second Generation Model. We have focused on developing a link between agricultural output of the Second Generation Model and our protein available parameter in Child Health and Welfare Index and are continuing to develop the linking algorithm.

Figures 1 and 2 show the output from World-CHWI and U.S.-CHWI.

\section{Publication}

Longstreth, J. 1993. "Health Effects of Global Warming: Problems in Assessment." Air and Waste Management Assoc $J$, in press. 


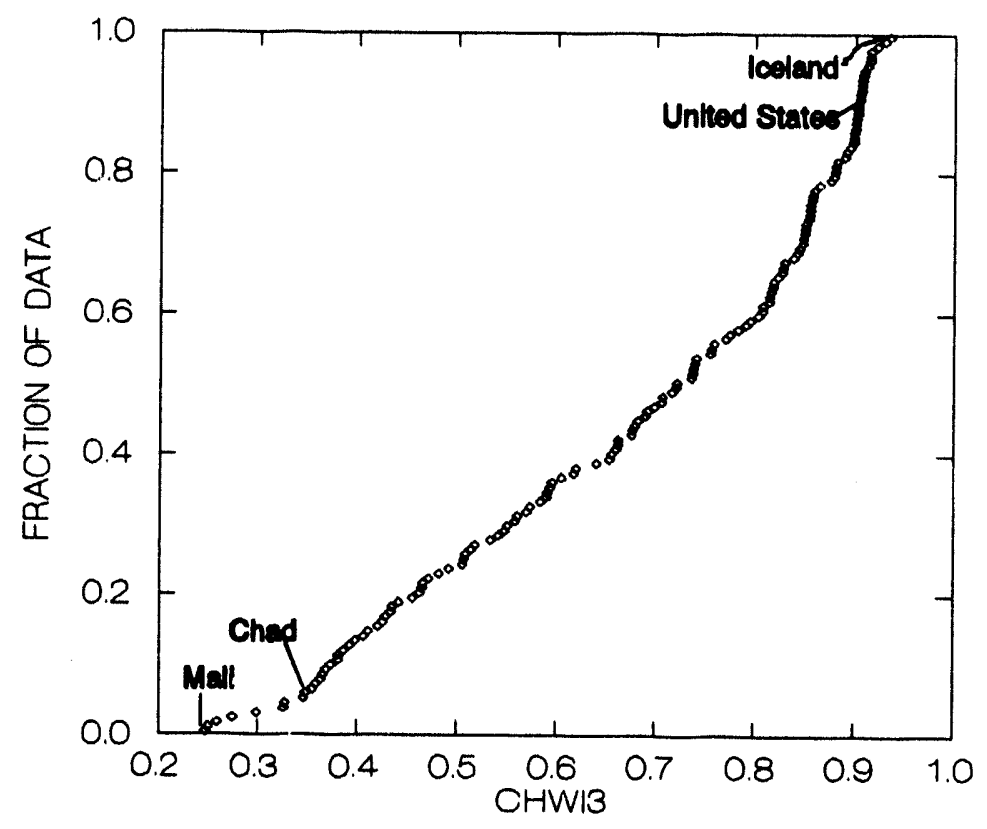

Figure 1. Child Health and Welfare Index

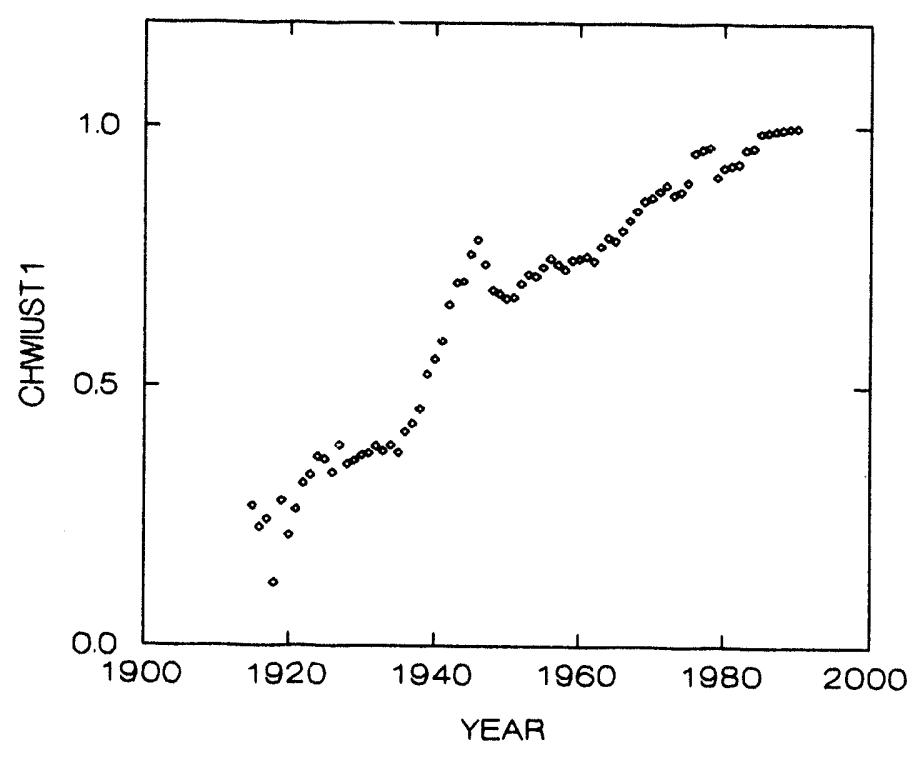

Figure 2. Temporal Variation in CHWI for the U.S., 1920-1990 


\title{
Computational Modeling of Complex Physical Systems
}

\author{
E. W. Pearson (Analytic Sciences)
}

This project is providing greatly enhanced capabilities for modeling coupled atmospheric transport and chemistry, subsurface transport, watersheds, coupled heat and mass transfer phenomena, and fundamental fluid dynamics. These capabilities are represented in the form of computationally efficient, state-of-the-art computational models. These models exploit massively parallel computing architectures and advanced numerical methods, and incorporate more realistic physics than current generation models.

Spatially-Distributed Hydrologic Process Modeling for Heterogeneous Watersheds (L. W. Vail, M. S. Wigmosta, J. L. Stroh)

High-resolution simulations of spatially-distributed hydrologic processes at the large watershed or regional scale are limited by conventional computing resources. To overcome some of these limitations, we have ported the serial version of PNL-WATERSHED to the massively parallel Connection Machine CM-2. The parallel version of the model is capable of running 26 times faster than the serial version. Such an increase in performance will allow us to expand our capabilities in the modeling of complex regionalscale hydrologic systems.

As background, the PNL-WATERSHED model provides an integrated representation of hydrologyvegetation dynamics at the topographic scale described by digital elevation data (nominally 90 meters in the north-south direction). It includes a two-layer canopy model for evapotranspiration, an energy-balance model for snow accumulation and melt, a two-layer rooting zone model, and a saturated subsurface flow model. Digital elevation data are used to model topographic controls on incoming shortwave radiation, precipitation, air temperature, and down slope water movement. At each time step, the model provides a simultaneous solution to the energy and water balance equations for each grid cell in the watershed. Individual grid cells are hydrologically linked through a quasi three-dimensional saturated subsurface transport scheme. A distinguishing feature of the model is that down slope redistribution of soil moisture via the saturated subsurface transport scheme is explicit, that is, accounting is done on a pixel-by-pixel basis, in contrast with the statistical representation used, for instance by TOPMODEL (Beven and Kirkby 1979). Computational efficiency of the subsurface model is attributable to the numerical scheme described by other researchers.

The massively parallel version of PNL-WATERSHED offers several distincl advantages over the serial version in meeting our present and future needs. Several current and planned studies require increased computational performance. These include

- A better representation of orographic effects on wind and precipitation fields. As our previous work has shown, this capability is critical in modeling the hydrology of mountainous watersheds.

- Improved sophistication of certain modeled processes, such as snow accumulation and melt.

- Uncertainty analysis of model simulations.

- Development of methods to incorporate the effects of topography and the spatial distribution of vegetation into a better parameterization of BATS.

Through this research, we have successfully enhanced our staff expertise in the parallel modeling of complex physical systems.

Parallel Implementation of PNL's Global Chemistry Model on the Intel Touchstone Delta Supercomputer (R. C. Easter, R. D. Saylor)

During FY 1993, a new chemical mechanism has been implemented into the Global Chemistry Model (GChM) that focuses on carbon monoxide/methane chemistry in the troposphere. Development of this new version of the Global Chemistry Model has been completed and initial simulations were performed for 
the September 1 - October 15, 1984, time period which coincides with the National Aeronautics Space Administration Measurement of Air Pollution from Satellites (MAPS) experiment. These simulations employed a relatively coarse grid resolution of $3.75^{\circ}$. Initial comparisons have been made between Global Chemistry Model results and global, mid-tropospheric carbon monoxide measurements taken as part of the Measurement of Air Pollution from Satellites. Results from these initial coarse-grid simulations are encouraging; simulations will next be performed using a finer grid resolution of $1.875^{\circ}$ and a longer initialization period.

During FY 1993, the conversion of the Global Chemistry Model from a sulfur model to a carbon monoxide/methane model took considerably more effort than was originally anticipated. Consequently, only limited progress was made in developing load balancing techniques for Global Chemistry Model simulations on the Intel Delta.

\section{Computational Investigation of Subsurface Heterogeneity (S. B. Yabusaki, T. D. Scheibe)}

We have improved our ability to model subsurface heterogeneity, and added particle tracking and associated visualization capabilities to our code. The code, which runs on a Connection Machine, has been demonstrated through application to a model problem on a 900-million node "numerical aquifer." Scaling results have been derived from model results that will permit improved accuracy in large-scale hydrologic modeling.

\section{Engineering Applications of Computational Fluid Mechanics (D. S. Trent, G. I. Fann, A. S. Koontz)}

During FY 1993, we focused on application of recent developments in numerical analysis to enhance TEMPEST code performance, and on development of a parallel version of TEMPEST for execution on multiple workstation central processing units. We have adapted the current TEMPEST input data structure to be suitable for parallel processing. This permits current TEMPEST input, with a few added lines, to execute a prototype parallel version of
TEMPEST. Enhanced code performance has also been demonstrated through the introduction of an improved solver to TEMPEST. Through use of multi-level blocked ILU preconditioned Generalized Minimal Residual linear equation solver, we demonstrated performance 1.5 times faster than the current TEMPEST solver on large problems on serial machines. Work continues on the parallel solver.

\section{Development of Direct Numerical Simulation Techniques (J. C. Phillips)}

Direct numerical simulation techniques were used to perform initial transition and turbulence calculations of the unstratified double-glazing flow problem. The numerical algorithm has been implemented on the Intel Touchstone Delta architecture and exhibits very promising parallel performance.

Three different cases were considered as initial tests of the code.

1. A decay of a "gray" noise perturbation of the conduction regime back to a quiet conduction regime.

2. The second case considered a supercritical growth of an oblique disturbance into a steady cellular convection state. Apparently, transverse disturbances in the oblique wave may grow rapidly to become a dominant part of the transition between the steady one-dimensional conduction regime and a steady multidimensional cellular state. This result has not been previously computed to our knowledge. Further calculations will have to be performed to validate the existence of this type of transition.

3. The third case is a supercritical transition to a turbulent convection state and an examination of the turbulence statistics of the flow.

\section{Reference}

Beven, K. J., and M. J. Kirkby. 1979. "A Physically Based, Variable Contributing Area Model of Basin Hydrology." Hydrological Sciences Bulletin 24:43-69. 


\title{
Computer Modeling of DNA Perturbations
}

\author{
J. H. Miller (Biology and Chemistry)
}

This project investigated changes in the structure and dynamics of DNA by the presence of lesions associated with free-radical attack from radiation exposure or other forms of oxidative stress.

Our understanding of the relationship between perturbations in DNA structure and changes in DNA function is currently limited by the difficulties in obtaining reliable atomic-resolution data on the structure and dynamics of perturbed DNA sequences. Recent advances in sample preparation, nuclear magnetic resonance spectroscopy, and computer technology forecast a significant increase in the availability of this type of information. This project used computer-based molecular modeling techniques to investigate the conformation of DNA sequences with potentially harmful genetic lesions.

Earlier work on the electronic structure of lesions formed by hydroxyl-radical (OH) attack on thymine was published in the International Journal of Radiation Biology. Extension of these ab initio quantum-chemical calculations to other ring-saturated pyrimidine lesions showed that the conformational preferences discovered for $\mathrm{OH}$ addition to thymine were also valid for cytosine and uracil. However, perturbations to DNA structure from $\mathrm{OH}$ addition to the $C(5)$ position of cytosine should not be as great as for thymine because native cytosine has a hydrogen substituent at $C(5)$ rather than a methyl group.

Addition of $\mathrm{OH}$ at $\mathrm{C}(5)$ forces the native ligand into an orientation that is perpendicular to the pyrimidine ring. A base modified in this manner is difficult to accommodate in the DNA double helix due to steric clashes with neighboring base pairs. These clashes should be more dramatic for thymine's bulky methyl group than they are for cytosine's hydrogen atom.

These speculations regarding the importance of nonplanarity in ring-saturated thymine lesions were confirmed by molecular dynamics simulation of the self-complementary DNA dodecamer

CGCGAATTCGCG, where the bold-faced $T$ denotes one of the following chemically-similar thymine lesions: 5,6-dihydrothymine (dhT) that has hydrogen additions to $C(5)$ and $C(6) ; 5$-hydroxy-5,6-dhT that has an $\mathrm{OH}$ adduct at $\mathrm{C}(5)$ and hydrogen addition to $\mathrm{C}(6)$; 6-hydroxy-5,6-dhT that has hydrogen at $\mathrm{C}(5)$ and $\mathrm{OH}$ at $\mathrm{C}(6)$; or 5,6-dihydroxy-5,6-dhT that has $\mathrm{OH}$ additions at both $\mathrm{C}(5)$ and $C(6)$. Substituting 5,6-dhT for native thymine did not significantly perturb the structure of the dodecamer. Similarly, $\mathrm{OH}$ addition to thymine at $\mathrm{C}(6)$ had only a minor effect. Both of the thymine lesions that contained $\mathrm{OH}$ at $C(5)$ cause significant DNA distortion due to the shift in the orientation of the native methyl group. These findings correlate well with the observed biological consequences of these ring-saturated thymine lesions.

Our studies of perturbations to DNA structure by 5,6dihydroxy-5,6-dhT (thymine glycol) provided a basis for investigating the mechanism by which the $E$. coli repair enzyme endowuclease III recognizes the presence of this lesion in DNA. Thymine glycol inhibits binding of endonuclease III to DNA containing oxidative base damage; hence, we speculate that the inhibitor and damaged DNA have the same binding site. This site has been identified from the crystal structure as a flexible loop on a surface of the enzyme with the correct electrostatic properties for attraction to DNA. Computer searches for the minimum-energy conformation of thymine glycol in this loop revealed that interactions with $C(5)$ and $C(6)$ hydroxyl groups are responsible for its binding as an inhibitor. These $\mathrm{OH}$ groups will lie in the major groove when thymine glycol is present in DNA; hence, they should be accessible for interaction with the binding loop. Based on this mechanism for damage recognition, we modeled a complex between endonuclease III and DNA containing thymine glycol and discovered DNAprotein interactions from alpha helices on the binding face of the enzyme that fit into the major groove of DNA above and below the lesion site. These results were highlighted in an invited paper at the conference DNA Damage: Effects on DNA Structure and Protein Recognition held recently in Burlington, Vermont. 


\section{Publications}

Miaskiowicz, K., J. Miller, and R. Osman. 1993. "Ab Initio Theoretical Study of the Structures of Thymine Glycol and Dihydrothymine." The International Joumal of Radiation Biology 63:677-686.

Miaskiewicz, K., J. Miller, and R. Osman, "Energetic Basis for Structural Preferences in 5/6-hydroxy-5,6dihydropyrimidines: Products of lonizing and UV Radiation on DNA Bases." Biochimica et Biophysica Acta, in press.

Miaskiewicz, K., J. Miller, R. Ornstein, and R. Osman, "Molecular Dynamics Simulations of the Effects of RingSaturated Thymine Lesions on DNA Structure." Biochemistry, submitted.

Miller, J., K. Miaskiewicz, and R. Osman, "Structure-Function Studies of DNA Damage Using Ab Initio Quantum Mechanics and Molecular Dynamics Simulation." In DNA Damage: Effects on DNA Structure and Protein Recognition, eds. S. S. Wallace et al., New York Academy of Sciences, New York, in press.

\section{Presentations}

Miller, J. 1993. "Energetics and Conformation of DNA Lesions: Effects of Solvation and DNA Environment." Presented at the 26th Radiological and Chemical Physics Contractors' Meeting, Chicago, nlinois.

Miller, J., K. Miaskiewicz, and R. Osman. 1993. "StructureFunction Studies of DNA Damage Using $a b$ initio Quantum Mechanics and Molecular Dynamics Simulation." Presented at the Conference on DNA Damage: Effects on DNA Structure and Protein Recognition, Burlington, Vermont. 


\title{
Contaminant Risk Evaluation and Management
}

\author{
E. A. Jenne (Geosciences)
}

An all-inclusive approach that covers human health-based rusk evaluation, remediation scenario development, costs of risk reduction, and public communication/perception was applied via this project, to provide DOE (and other agencies) with an exemplary, science-based, integrated method for estimating the risks from hazards from contaminants now present in the environment and encountered in remediation.

\section{Integrated Risk Assessment}

It was the intent of this project, in conjunction with an ongoing Hanford Site risk assessment activity, to provide high-level rationalization of approarhes and tools and provide integration logic and analysis. However, during the first quarter of this fiscal year, there was no ongoing risk assessment tied to a particular Hanford area. Therefore, this objective could not be realized and all efforts after mid-January were focused on the four research tasks described below.

\section{Mental Models}

A "mental models" hypothesis of the knowledge and perceptions of DOE contractor employees about exposure processes and effects of ionizing radiation was compared with an expert model of the same topics. A comparison regarding employee perceptions regarding work place chemicals was simultaneously investigated. Fifteen radiation workers from Westinghouse Hanford Company and the Pacific Northwest Laboratory were interviewed for this study. Their responses were compared with a science model to see where differences occurred. The conclusions of this research follow.

Applicability of Approach. The mental model approach is useful for investigating the knowledge and perceptions of radiation workers and comparing them with an "expert" science model to reveal differences. As part of this process, it is also helpful to elicit employee values, especially those that may influence behavior.

\section{Comparison of Worker and Science Models.}

Worker mental models of radiation exposure processes, controls, and effects were similar to the science model of the same topics, with some differences. Workers seemed aware of basic radiation exposure processes, controls, and effects. However, their mental models did not contain the level of detail that the science model did. For example, some workers were aware that there was a way to mitigate internal contamination via chelation, but no one mentioned concepts such as administering an antidote, inducing vomiting, gastric and pulmonary lavage, removing from wound, or irrigating with saline. Another example of less detail or lack of knowledge among workers was in factors that affect dose, such as weight, gender, and treatment level. No one mentioned bioassays as controls, though we assume many of our interviewees are required to participate in bioassay testing.

Comparison of Exposure Routes. Workers generally viewed internal radiation exposure as more of a concern than external exposure or skin contamination. The most commonly stated reasons for this concern were 1) the inescapability of an internal source, in contrast to shielding, protective clothing, and distance to control external exposure and skin contamination; and 2) the greater potential harm from an internal exposure, specifically, continued irradiation once inside the body and the tendency of internal contamination to seek organs and bone.

Estimated Probabilities of Risk and Harm. Workers did not judge their risks to be greater than those of their colleagues for either radiation exposure or for harm from that exposure. Half the subjects rated their probabilities of exposure as higher than for someone picked at random from the Hanford community, which could very well be true, in light of the much larger number of nonradiation versus radiation workers employed at Hanford.

Exposure Pathways. Workers believed they had the highest probability of receiving "terminal" exposure, with less chance of skin contamination and even less for internal irradiation. Subjects typically believed the 
probability of harm from internal irradiation was less than or equal to that from skin contamination or external irradiation. Interestingly, workers' written estimates of no greater harm from internal irradiation were at odds with the concerns they expressed in the narrative part of the interview about internal irradiation (described in Comparison of Exposure Routes above).

Values and Concerns. Many workers said they respect, but do not fear radiation, and accepted the radiation-related risks as part of their jobs. This attitude may reflect knowledge, experience, degree of personal control over the risks, and/or confidence in administrative and engineering controls.

\section{Workers Assess the Knowledge and Awareness}

Levels of Their Coworkers. For example, they show a strong reliance on the support and advice of the Health Physics technicians. On the other hand, several workers expressed a concern about colleagues' attitudes, including complacency or unwarranted fear, that can affect other peoples' exposure risks. Longterm workers were sometimes viewed as having a more casual attitude toward exposure, possibly because they had not experienced any noticeable health effects over several years of working around radiation. Another concern was unreasonably restrictive dose limits. Half the workers said that cancer and other serious diseases have too many causative factors to be attributed to their radiation exposure at Hanford. However, two people ranked the percent chance at $100 \%$ and $50 \%$, respectively, of radiation exposure being the cause of a such a disease.

Chemicals. Most of the interviewees encountered hazardous chemicals in the work place. Specific chemicals mentioned were asbestos, ammonia, polychlorinated biphenyls, mercury, carbon tetrachloride, nitric acid, lead, alcohol, ferrocyanide, cyanide, nitric fumes, acids, and bases. A variety of potential exposure effects were stated, ranging from watering eyes to organ damage and coma. Many control methods were described, including administrative and physical. Interestingly, three workers stated that chemicals were more dangerous in the work place than radiation was, and two felt that chemicals received less emphasis than radiation did at Hanford.

Training. All subjects felt radiation training was useful because it helped them do their jobs properly and safely. This response could be partly attributed to the fact that our subjects were relatively new in terms of time qualified as radiation workers (5 years or less). Workers recommended changes in frequency and length of training, targeting of workers, applicability to the work place, and instructional methods.

\section{Lost Life Expectancy}

Lost life expectancy rate is the amount of expected life shortening that occurs per unit time under a given exposure condition (that is, seconds of life shortening per second of exposure, years per year, etc.). For managing risks in the work place and the environment, limits are currently placed on concentrations or dose rates or analogous quantities for other energy fields. Current regulatory benchmarks, such as threshold limit values or permissible exposure levels, are shown to be inadequate for expressions of risk due to differences in units (e.g., $\mathrm{mg} \mathrm{m}-3$ and parts per million), and differences from one agent to another in lifetime fatal cancer risk per unit time exposed at one threshold limit value or one permissible exposure level. Lost life expectancy rate is superior to current regulatory approaches in that it provides a single, dimensionless risk number that expresses risk from all agents producing stochastic effects.

Lost life expectancy rate can be used as a risk communication tool to express and compare exposures to multiple agents. For stochastic effects, risk estimates may apply to populations rather than to individuals. Nevertheless, individuals ask about risk, and we are required to train workers about the health risks of radiation on the job. To be individual-specific, one may adjust the lost life expectancy rate calculation for age, sex, smoking status, future parent status, and other known risk and effect-modifying factors.

\section{Carcinogenicity and Teratogenicity of Combined Insults Relative to Mechanisms of Toxicity}

Limited paradigms exist for predicting the nature and magnitude of effects produced by interactions among two more insults or for relationships between the types of toxic effects that they produce. Pertinent information has been extracted from four independent data bases to establish the extent to which a given agent causes both carcinogenic and teratogenic effects. Biological response data is often subjective and qualitative. However, a basis has been developed for predicting meaningful estimates of risk from commonalities associated with multisystem responses. The underlying mechanisms that could explain the interactions between interactions that have been sought. It is 
hypothesized that teratogenesis and carcinogenesis often occur through different mechanisms and/or act on different targets. The underlying basis for this expectation has been developed in terms of ccmparisons of the teratogenic sequela that result from altered developmental processes which also result in tumor formation, as well as in abnormalities of tissue repair-mammalian toxicities of organic substances with effects considered to be primarily environmentally relevant lesions. This analysis is expected to result in a refereed journal article.

\section{Multivariable Modeling of Toxicant Acute Lethality to Fish and Other Aquatic Organisms}

Assuming that experimental organisms are physiologically identical and their mortalities are independent, the number of fish or other organisms that have expired by time $t$ at aqueous toxicant concentration $X$ is distributed as binomial $\operatorname{Bin}\left(25, P_{t, X}\right)$, e.g., where $P_{t, X}$ is the

\section{Prob [Number of Expired Fish $=S]=$}

probability of mortality of a single fish by time $t$ at an aqueous metal concentration $X$. This probability is a non-decreasing function of buth $t$ and $X$.

The approach used was to represent $P_{t, X}$ as:

$P_{t, x}=\frac{e^{g(t x)}}{1+e^{g(t x)}}=\frac{1}{1+e^{-g(t, x)}}$

where

$g(t, x)=B_{0}+b_{1} g_{1}(g, x)+\ldots+b_{p} g_{p}(t, x)$

and obtain the values $b_{i}$ that maximize the likelihood function $L\left(b_{0}, \ldots, b_{p}\right)$

$L\left(b_{0}, \ldots, B_{p}\right)=\prod_{i=1}^{k}\left(\begin{array}{c}25 \\ s_{1}\end{array}\right) P_{i, x_{1}}^{s_{1}}\left(1-P_{i, x_{1}}\right)^{25-s_{i}}$

where $\mathbf{k}$ is the total number of observation times over all concentration levels. Let $b$ be the vector of coefficients $\left(b_{0}, \ldots, b_{p}\right)^{T}$. The likelihood function $L(b)$ is nonlinear in $b$, so obtaining the vector $b^{*}$ that maximizes $\mathrm{L}$ must be via a nonlinear optimization procedure. Although they are not guaranteed to provide good initial values, the least-squares estimates $b_{1 \mathrm{~s}}$ are used as initial value of $b$. The least-squares values are obtained as follows: let $p_{i} i=1, \ldots, k$ be the observed mortality percentages. These $p_{i}$ can be transformed to obtain the negative log ratios. Setting the $p_{i}$ equal to the function $P\left(t_{i}, X_{i}\right)$, this transformation is found to be linear in the coefficient vector $b$.

To predict the number of fish killed in another experiment after $t$ hours at a given aqueous toxicant concentration, multiply the predicted proportion by the total number of fish in the experimental system by the number of fish, N. Mortality results from the zinc experiment and those from the cyanide experiment were each regressed against the respective total dissolved concentrations, time, and time-concentration interaction terms in the forms of the following model:

$P_{t, x}=\frac{1}{1+\exp ^{b_{0}+b_{t h}(x)+b_{2} \ln (t)+b_{g} \ln (x) \ln (x)}}$

wherein $t$ is time (day) and $X$ is either total zinc concentration or total cyanide concentration, depending upon which response data are being considered. Each of the models had an (uncorrected) R-square value that was in excess of 0.97 . The single toxicant mortality data were combined with the joint zinc and cyanide mortality data, and an overall model was fit to these combined data. The single-toxicant data were augmented to include the absent toxicant by assigning a zero concentration to the missing toxicant, and these data were then applied to the joint toxicant results. Although the final parameter estimates were obtained via maximum likelihood estimation, an $R$-square value of $\mathbf{0 . 8 2}$ was tabulated to provide an indication of model fit.

The equations above were used to predict zinc $\mathrm{LC}_{50 \%, 96 \mathrm{hr}}$ from literature data. The only literature data sets available to test the model were for other species. These models reproduced experimental literature data within an order of magnitude. Much of the discrepancy results from the necessity of using mortality data for different species to test the model. The results are considered promising. 


\title{
Determination of the Potential for Using Genetic and Physical Biomarkers of the Big Sagebrush as a Tool for Investigating Environmental Change
}

\author{
R. J. Fellows (Environmental Sciences)
}

The objective of this project was to determine genetic (DNA alterations) and morphological (allometric) characteristics of big sagebrush (Artemisia tridentata) growing at the opposite margins of its range in the Snively Basin of the Hanford Reservation's Arid Lands Ecology Reserve. Measurements will include tissue removal for DNA isolation and characterization with appropriately developed molecular probes, as well as nondestructive analysis of the physical stature of the plants and statistical analysis of the resulting data. Determination of genetic (molecular), as well as physical biomarkers reflective of environmental conditions will be performed.

The shrub-steppe ecosystem, which covers the majority of the land surface of the non-riparian areas of the Hanford Site, is the primary ecotype between the Cascade and Rocky Mountain ranges of the Pacific Northwest. This ecosystem will be extremely sensitive to projected elevations in temperature and reduced moisture regimes following global warming. Assessment of the degree of sensitivity and potential damage during this period will require the development of reliable stress bioindicators both on the organismal and molecular levels.

The primary biomass component of this ecosystem is the surface vegetation, specifically shrubs, such as big sagebrush (Artemisia tridentata). These shrubs are perennial, widely distributed, and some on the Hanford Site may live for more than a century with rooting depths in excess of several tens of meters. These plants may thus serve as integrators of environmental stres? over all media and across a number of years. Elevational gradients across the range of the sagebrush on the Hanford Reservation's Arid Lands Ecology Reserve (ALE) provide in situ climatic variations wherein physical and molecular responses may already be evident.
The work initiated during this fiscal year has included the physical (allometric) assessment of sagebrush communities at altitudes of 620 meters above mean sea level (termed "Upper Snively") and 280 meters (termed "Lower Snively"), the collection of leaf tissue from these sites, development of applicable DNA extraction techniques for this tissue, the generation of phage libraries from this DNA, and the cloning and sequencing of potential molecular probes.

\section{Allometric Assessment for Probe Selection}

Measurements taken at the two sites included plant height, canopy width along major and minor axes, and distance from canopy to canopy between the shrub and that of the nearest neighbor. Following this, one main branch from each plant was collected and returned to the laboratory where measurements on branches were taken on three subbranches and were confined to the terminal three internodes at each subbranch. These measurements were total number of minor branches, number of leaves in each of five size classes ( $<4 \mathrm{~mm}, 4$ to $8 \mathrm{~mm}, 8$ to $10 \mathrm{~mm}, 10$ to $13 \mathrm{~mm}$, and $>13 \mathrm{~mm}$ ), diameter at center of internode, and internode length.

The results, presented in Tables 1 and 2 show that big sagebrush plants from Lower Snively were smaller than plants from Upper Snively, although the difference was not significant at the $95 \%$ confidence level (Table 1). Plants were significantly more crowded in Lower Snively than in Upper Snively although there was no significant relationship between crowding and shrub volume (Interaction effect: nearest neighbor distance $x$ location, $F_{1,14}=1.55, P=0.23$ ).

Sagebrush in Upper Snively had significantly fewer minor branches off each subbranch, but had significantly more leaves in the largest size class than did plants from Lower Snively (Table 2). The increased minor subbranching in the Lower Snively plants did not lead to increased photosynthetic material, because 
there were no significant differences in total leaf number between the two areas. Under conditions of water stress, sagebrush plant excise large leaves and replace them with the smaller leaves. Plants in the Upper Snively microclimate were, therefore, probably under less water stress than plants in the Lower Snively microclimate.

Internodes of plants in Upper Snively were generally longer and of larger diameter than were internodes of plants in Lower Snively (Table 2), indicating that Upper Snively plants were allocating more resources to above-ground growth than were plants at the Lower Snively location. This result is consistent with the general water stress hypothesis. Differences in length of the terminal internode were less between the two sites during the most recent year, consistent with the 1992-1993 weather, which was the wettest on record. These observations indicate that internode length and diameter may be an accurate whole plant level bioindicator of stress in big sagebrush.

\section{Molecular Probe Development}

There were no DNA extraction/purification techniques available in the literature for sagebrush. Therefore, the initial efforts were directed at adapting and refining protocols available for other plant species. One of these, described by Rogers and Bendich (1988) employed cetyltrimethylammonium bromide (CTAB) in the extraction buffer. After several attempts with this method, DNA yields averaged less than $1 \mu \mathrm{g} / 10 \mathrm{~g}$ fresh weight of leaves. Another method described in Current Protocol (1993) employed repetitive $\mathrm{CsCl}$ gradient centrifugations and consistently provided DNA yields of 50 to $100 \mu \mathrm{g} / 10 \mathrm{~g}$ of sagebrush leaves. This latter method was employed to obtain sufficient $(100 \mu \mathrm{g})$ genomic DNA to start the low- $\mathrm{C}_{0} \mathrm{t}$ library.

The DNA was then sheared ultrasonically to 0.3 to $2.0 \mathrm{~Kb}$ fragments and the double- and single-stranded DNA fragments separated on an hyroxylapatite column. Double-stranded DNA eluted from the column was found to contain primarily the repetitive sequences. Reassociation kinetics for these repetitive fragments provided a $C_{0} t$ value of 0.5 . The low $C_{0} t$ DNA fragments were then blunt ended, ligated into the plasmid pUC 18, and, after four initial failures, successfully shown to transform $E$. coli JM 109 cells.

Fifty colonies have been generated from the sagebrush low $\mathrm{C}_{\mathrm{o}} \mathrm{t}$ library. Minipreparation of all $\mathbf{5 0}$ colonies were performed which consisted of cutting each of the colonies DNA with EcoR 1 and Bam H1 to check the size of each fragment. As of this time, three of the colonies have been subjected to sequencing and the initial data employing the forward primer are presented in Figures 1, 2, and 3. Sagebrush specific probes may be generated from these sequences to determine genetic differences between plants known to exhibit significant allometric variations.

During this initial year several project goals were realized. Allometric measurements were successful in identifying the potential whole plant biomarkers of internode distance and diameter in big sagebrush. Further, confirmation of these should be performed at a site intermediate to the two elevational extremes chosen. Big sagebrush DNA has been isolated and genetic probes have been constructed. Probe efficiency and direct correlations with confirmed physical parameters remain to be accomplished.

\section{References}

Ausubel, F. M., R. Brent, R. E. Kingston, D. D. Moore, J. G. Seidman, J. A. Smith, and K. Struhl, eds. 1993. Current Protocol. J. Wiley \& Co., New York.

Rogers, S. O., and A. J. Bendich. 1988. "Extraction of DNA From Plant Tissues." In Plant Molecular Biology Manual A6:1-10, eds. S. B. Gelvin, R. A. Schilperoort, and D.P.S. Verma. Kluwer Academic Publishers, Dordrecht, The Netherlands. 
Table 1. Analysis of Variance Results Comparing Piant Characters at Two Locations

\begin{tabular}{|c|c|c|c|c|c|}
\hline Character & $\mathbf{F}$ & df & $\mathbf{P}$ & $\begin{array}{c}\text { Lower Snively } \\
\text { Mean } \\
\end{array}$ & $\begin{array}{c}\text { Upper Snively } \\
\text { Mean } \\
\end{array}$ \\
\hline Volume & 2.33 & 1,16 & 0.15 & $0.39 \mathrm{~m}^{2}$ & $0.61 \mathrm{~m}^{2}$ \\
\hline Nearest neighbor & 5.58 & 1,16 & 0.031 & $9.9 \mathrm{~cm}$ & $21 \mathrm{~cm}$ \\
\hline
\end{tabular}

Table 2. Nested Analysis of Variance Results Comparing Subbranch Characters at Two Locations

\begin{tabular}{|c|c|c|c|c|c|}
\hline Character & $\mathbf{F}$ & df & $\mathbf{P}$ & $\begin{array}{c}\text { Lower Snively } \\
\text { Mean } \\
\end{array}$ & $\begin{array}{c}\text { Upper Snively } \\
\text { Mean }\end{array}$ \\
\hline $\begin{array}{l}\text { Subbranch } \\
\text { \# Minor Branches } \\
\text { \# Leaves } \\
\text { \# Leaves >13 mm }\end{array}$ & $\begin{array}{l}9.21 \\
0.139 \\
10.2\end{array}$ & $\begin{array}{l}1,16 \\
1,16 \\
1,16\end{array}$ & $\begin{array}{l}0.008 \\
0.7 \\
0.006\end{array}$ & $\begin{array}{l}10.6 \\
61 \\
10.9\end{array}$ & $\begin{array}{l}8.3 \\
64 \\
24.8\end{array}$ \\
\hline $\begin{array}{l}\text { 1st (terminal) Internode } \\
\text { Length } \\
\text { Diameter }\end{array}$ & $\begin{array}{l}0.226 \\
8.19\end{array}$ & $\begin{array}{l}1,16 \\
1,16\end{array}$ & $\begin{array}{l}0.6 \\
0.01\end{array}$ & $\begin{array}{r}54.6 \mathrm{~mm} \\
2.4 \mathrm{~mm}\end{array}$ & $\begin{array}{r}58.7 \mathrm{~mm} \\
2.8 \mathrm{~mm}\end{array}$ \\
\hline $\begin{array}{l}\text { 2nd Internode } \\
\text { Length } \\
\text { Diameter }\end{array}$ & $\begin{array}{l}4.75 \\
8.19\end{array}$ & $\begin{array}{l}1,16 \\
1,16\end{array}$ & $\begin{array}{l}0.045 \\
0.01\end{array}$ & $\begin{array}{r}35.4 \mathrm{~mm} \\
2.4 \mathrm{~mm}\end{array}$ & $\begin{array}{r}47.9 \mathrm{~mm} \\
2.8 \mathrm{~mm}\end{array}$ \\
\hline $\begin{array}{l}\text { 3rd Internode } \\
\text { Length } \\
\text { Diameter }\end{array}$ & $\begin{array}{l}4.95 \\
3.03\end{array}$ & $\begin{array}{l}1,16 \\
1,16\end{array}$ & $\begin{array}{l}0.041 \\
0.10\end{array}$ & $\begin{array}{r}28.0 \mathrm{~mm} \\
2.9 \mathrm{~mm}\end{array}$ & $\begin{array}{r}47.9 \mathrm{~mm} \\
3.3 \mathrm{~mm}\end{array}$ \\
\hline
\end{tabular}




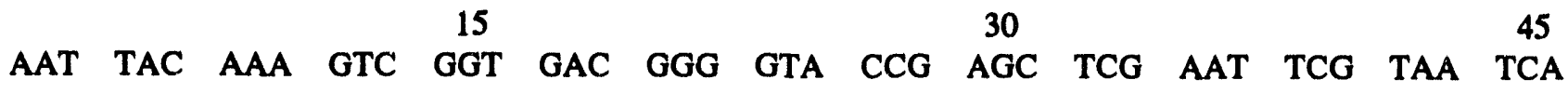

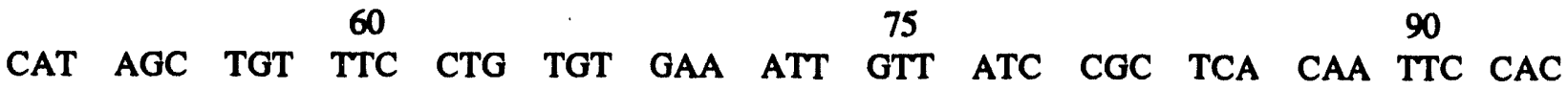

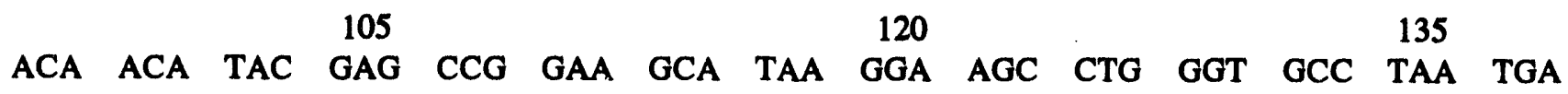
GTG AGC TAA CTA CAT TAT GCG TG

Figure 1. Sagebrush Colony \#3 Sequence with Forward Primer

$15 \quad 30 \quad 45$

TAT CGT AAT CAT GTC ATA GCT GTT TCC TGT GTG AAA TTG TTA TCC GCT CAC AAT TCC ACA CAA CAT ACC GAG CCG GAA GCA TAA AGT GTA AAG CCT GGG GTG CCT AAT GAG TGA GCT AAC TCA CAT TAT TGC $\begin{gathered}135 \\ \text { GTT GCT CTC ACT }\end{gathered}$ 150

GCC GCT TTC AGT

Figure 2. Sagebrush Colony \#4 Sequence with Forward Primer

$15 \quad 30 \quad 45$

ATg Gag TGA AGA TGg ATC TTG TAg AGT TGG GGA AGT ACG TAC GCC ACC CAC GAT TGg TAT TAA GGA ATA CCC CGT CAT CCA CGC GAC TAA TCC AGg

GTC CAG GCT CGA ATT GTA TAT GTC AGC TGT CTG TGT GAA TGT ATC

Figure 3. Sagebrush Colony \#5 Sequence with Forward Primer 


\title{
Development of Advanced Flow Cytometry Methods for Investigating EMF-Induced Calcium Ion Mobility
}

\author{
J. E. Morris (Biology and Chemistry)
}

Recently acquired flow cytometry at PNL provides a unique capability to investigate cellular mechanisms involved in electric and magnetic effects on biological tissue. This project resulted in direct interfacing of magnetic field exposure systems to the cytometer, and served as a precursor for detailed mechanistic studies on effects of environmental agents at the cellular level.

Calcium ion mobility has become a central focus of efforts to determine possible mechanisms of electromagnetic field (EMF) interaction with biological systems. Properly interfaced to magnetic field exposure systems, flow cytometry provides opportunities to investigate, in near real-time, iree calcium cystosolic levels in exposed cells. Near real-time measurement capability will be crucial to understanding cellular level mechanisms of electromagnetic field exposure because of the rapid response of cellular elements to electromagnetic field exposure.
The major objectives of the project have been realized and include the following: 1) design, fabrication, and testing of an in vitro exposure and sampling system; 2) interfacing of the sampling system with the fluidics of the flow cytometer; and 3) establishing methods for acquiring and storing the data sets generated during the 1-hour exposure periods. The additional work element completed during this year was directed at the completion of modifications and repairs of the $60-\mathrm{Hz}$ magnetic field exposure system and the acquisition of calcium levels durir'g, different electromagnetic field exposures and frequencies.

The approach involves loading mammalian cells with an Indol-1 dye and exposing them to various intensities and frequencies of electromagnetic fields. The dye undergoes a substantial shift in absorption spectrum based on the amount of bound calcium. This approach provides an order-of-magnitude or more increase in sensitivity compared to existing methods. As noted previously, the near real-time assessment of cystosolic calcium levels during exposure will provide a method for the detailed investigation of cellular mechanisms. 


\title{
Development of a Regional Scale Risk Computation Capability for Assessment of Global Environmental Change Impacts
}

\author{
J. G. Droppo (Earth and Environmental Sciences)
}

The increasing levels of development occurring throughout the world are likely to be accompanied by a corresponding increase in the emissions of trace metals and other pollutants into the atmosphere. These pollutants, which are subject to both short- and long-range atmospheric transport, are rapidly becoming a global environmental problem, which is likely to grow in magnitude and significance as development progresses. The objective of this project was to refine and further develop the algorithms in the Multimedia Environmental Pollutant Assessment System (MEPAS) and RATCHET for screening-level assessment of human health and ecological regional scale impacts.

A recent PNL report by Friedman, Ashton, and Rapoport (1993) indicates that anthropogenic emissions of heavy metals into the atmosphere may result in significant adverse ecological and human health effects. These authors recommend research on a global scale using a holistic approach that focuses on deposition processes in individual regions.

Regional scale risk assessment modeling capabilities are needed for use in screening studies to determine direction for detailed investigation of potential impacts of development, fuel usage, etc., on the environment. These physics-based models should use readily available data, be easy to use, simple, and computationally efficient. PNL has models, such as the Multimedia Environmental Pollutant Assessment System (MEPAS), that when modified and linked will meet these criteria for use in its Global Studies Program.

\section{Regional Scale Aquatic Ecological Impacts}

This effort conducted with PNL staff member, R. E. Hegner, involved evaluation of regional-scale ecological impacts of trace metals and other hazardous air pollutants deposited in aquatic ecosystems. A screening-level model component is being developed for making estimates of potential regional-scale ecological impacts from projected increases in the atmospheric emissions of trace metals.

During FY 1993, candidate models for computing trace metal water concentrations were reviewed. A previously developed MEPAS lake model was felt to be too detailed for this application. Other models met some of the needs, but no model was found that met the appropriate level of detail necessary for this application.

The decision was made to create a new model component with the desired level of detail. The formulations for this new model were developed and implemented as a FORTRAN code on a personal computer. Test runs were made to compare with trace metal field studies data. Although good comparisons were obtained between predicted and measured concentrations, an ambiguity in the units for the reported literature values will need to be resolved before these results can be published. The model formulations and testing results are documented in a draft report, $A$ Model for Predicting the Biophysical Cycling of Atmospherically-Derived Mercury in Shallow, Temperate-Done Seepage Lake Systems, by S. T. Hwang, R. E. Hegner, and J. G. Droppo Jr.

\section{Regional-Scale Trace Metal Impacts}

This effort conducted with PNL staff member J. V. Ramsdell involves the development of regional risk assessment capabilities for long-range human health impacts resulting from projected releases of trace metals and other hazardous air pollutants. Currently available models such as the PNL code MEPAS consider population impacts only in the immediate region surrounding the release. To fulfill the need for extended regional risk computations, a longer-range transport code is to be linked to MEPAS risk computation capability.

During FY 1993, the regional scale dispersion and deposition model was ported from a UNIX workstation to the DOS platform and tested. The program was modified to use synoptic scale meteorological data 
inputs. Output products compatible with the input requirements of MEPAS were added. The meteorological data for making extended regional runs in Asia were obtained and test runs were made with that data. A typical simulation of a 12-month period takes about 1 hour $0 .: 386$-based computer.

\section{References}

Friedman, J. R., W. B. Ashton, and R. D. Rapoport. 1993. "A Review of the Global Emissions, Transport, and Effects of Heavy Metals in the Environment." PNL-SA-22394, Pacific Northwest Laboratury, Richland, Washington. 


\section{Elastic Properties, Solution Chemistry, and Electronic Structures of Oxides, Silicates, and Carbonates}

D. M. Sherman (Theory, Modeling and Simulation)

\begin{abstract}
The first-principles calculation of the equations of state of oxides, silicates, and carbonates was investigated. The results of this work were used to assess the reliability of emerging theoretical methods being applied to the study of mineral surfaces. Such an assessment is needed to provide a foundation for theoretical work in environmental geochemistry. Future work will apply quantum chemistry to develop a new approach to understanding and predicting the solid-solution chemistry of these phases.
\end{abstract}

During FY 1993, several systems were investigated using the periodic Hartree-Fock approach and the linearized augmented plane wave (LAPW) density functional method. Emphasis was placed on highpressure phases since these provide an opportunity to compare theory with experimentally measured equations of state. The results obtained here are the first which demonstrate the reliability of treating ionic minerals at the Hartree-Fock level.

\section{Stishovite and Other $\mathrm{SiO}_{2}$ Phases}

The electronic structure, equation of state, and pressure-induced phase transitions of stishovite were calculated using periodic Hartree-Fock theory. Using a moderately extended basis set, the calculated equation of state is in close agreement with experimental results taken under hydrostatic conditions. Moreover, the calculated equation of state is very close to that recently obtained using the linearized augmented plane wave method. The possibility that stishovite might transform to a denser phase at high pressure is investigated by calculating the free energy of $\mathrm{SiO}_{2}$ in the modified fluorite and $\mathrm{a}-\mathrm{PbO}_{2}$ structures. The enthalpy of $\mathrm{SiO}_{2}$ with the modified fluorite structure is too high to allow that phase to have any stability in the earth's interior. The $0 \mathrm{~K}$ enthalpy of silica in the $\mathrm{a}-\mathrm{PbO}_{2}$ structure is within $10 \mathrm{~kJ} / \mathrm{mol}$ of that of stishovite and a transition to such a structure cannot be ruled out in the temperature regime of the lower mantle. These results are in good agreement with the experimentally observed behavior of stishovite.

\section{Perovskite Phases}

The cubic $\mathrm{CaSiO}_{3}$ perovskite phase is possibly the third most abundant mineral in the earth. In addition to its geochemical significance, $\mathrm{CaSiO}_{3}$ perovskite provides a useful system with which to test the application of quantum chemistry to silicates. The equation of state and elastic properties of cubic $\mathrm{CaSiO}_{3}$ perovskite were calculated using the periodic Hartree-Fock formalism with several different basis sets. Calculations using a moderately extended basis set give an equation of state for $\mathrm{CaSiO}_{3}$ in close agreement with experiments. The free energy, as a function of pressure, of stishovite and $\mathrm{B} 2-\mathrm{CaO}$ were calculated at the same level of theory. From those results, it is found that cubic $\mathrm{CaSiO}_{3}$ perovskite is stable relative to the free oxides at pressures up to $130 \mathrm{GPa}$ (the core-mantle boundary). The solid solution behavior in the $\mathrm{MgSiO}_{3}-\mathrm{CaSiO}_{3}$ system was calculated and it was found that the two perovskites will be immiscible below their melting temperatures. This is in agreement with the apparent phase equilibria observed using high-pressure experiments. The calculated energy strain curves in these systems will be used to parameterize interatomic potentials for lattice dynamics simulations.

\section{Iron and Iron Oxides}

Many fundamental issues about the electronic structures of solids arise in the high-pressure behavior of iron and iron oxides. The nature of a new highpressure phase of iron observed in shock-wave experiments at Los Alamos (Brown and McQueen 1982) was addressed from linearized augmented plane wave band structure calculations. Electronic structure calculations show that the new phase may in fact be BCC iron that is stabilized under shock temperatures by the higher magnetic and electronic entropy of BCC iron over that of FCC and HCP iron. The incorporation of oxygen and sulfur into metallic iron was investigated 
from calculations of the static free energy of $\mathrm{Fe}_{3} \mathrm{~S}$ and $\mathrm{Fe}_{3} \mathrm{O}$. Pressure does not enhance the ability of oxygen to form a substitutional solid solution with iron. Sulfur, however, behaves very differently so that complete solid solution between Fe and FeS at extreme pressure is predicted. These results bear on some important current issues in geochemistry and demonstrate how quantum chemistry can play a role in the Earth Sciences.

\section{Boehmite}

Boehmite provides a useful analogue to more complex hydrous minerals. The nature of hydrogen bonding in minerals is of fundamental interest. In phases with strong hydrogen bonds, protons (hydrogen ions) may lave an enhanced mobility for intersite hopping and ome delocalized. Both pressure and temperature $\Rightarrow$ expected to enhance proton mobility. Since mubile protons can act as charge carriers, hydrous minerals might make a significant contribution to the electrical conductivity of the earth's mantle or lower crust. There have been several studies indicating that protons in boehmite ( $\mathrm{g}-\mathrm{AlOOH}$ ) are delocalized. In this work, the electronic structure of boehmite was calculated using periodic Hartree-Fock theory together with density functional estimates of the correlation energy. The optimum proton positions were determined and it was found that boehmite has space group Cmcm but with hydrogens occupying $50 \%$ of the $8 f$ sites.

The barrier for proton hopping between adjacent oxygens was determined to be $0.67 \mathrm{eV} / \mathrm{H}$ atom at the Hartree-Fock level. The energy of this barrier was corrected for electron correlation using the local density approximation but it was found that, in the local density approximation, correlation did not affect the barrier.

\section{References}

Brown, J. M., and R. G. McQueen. 1982. "The Equation of State of Iron and the Earth's Core." In High Pressure Research in Geophysics, pp. 611-625, eds. Akimoto S. and M. Manghnani, Center for Academic Publications, Tokyo.

Publications

Sherman, D. M. Electronic Structure, Entropy and the HighPressure Stability of BCC Iron. In: High Pressure Science and Technology 1993, eds. S. Schmidt et al. American Physical Society, Washington, D.C., in press.

Sherman, D. M. "Equation of State, Elastic Properties and Stability of CaSiO3 Perovskite: First Principles (Periodic Hartree-Fock) Results." Journal of Geophysical Research, in press.

Sherman, D. M. 1993. "Equation of State and High Pressure Phase Transitions of Stishovite, SiO2: ab Initio (Periodic Hartree-Fock) Results." Journal of Geophysical Research 98:11865-11873.

\section{Presentations}

Sherman, D. M. 1993. "Electronic Structure and Stability of Possible High-Pressure Fo-S-O Alloy Phases in the Earth's Core." Presented at the AIRAPT conference, Colorado Springs, Colorado (invited).

Sherman, D. M. 1993. "Equation of State and Stability of Lower Mantle Phases from First Principles." Presented at the EOS, Spring Meeting, American Geophysical Union (invited).

Sherman, D. M. 1993. "Quantum Chemistry of Mineral Surfaces." Presented at the Fall Meeting, American Geophysical Union (invited).

Sherman, D. M., H. J. Jansen. 1993. "High Pressure Electronic Structure of $a, g$ - and e-iron: The Nature of the Earth's Inner Core." Presented at the EOS, Proc. Spring Meeting, Americun Geophysical Union (invited). 


\title{
EMF Biological Effects: Characterization of Magnetic Fields in
}

\section{Homes}

\author{
N. H. Hansen (Automation and Measurement Sciences)
}

This project conducted measurement and analysis of magnetic fields using broad-band commercial electronics equipment to provide an initial data base on appliance-generated magnetic fields. The magnetic field data recorded were identified based on discussions with biology staff (at PNL) who are familiar with the proposed electromagnetic field biological effects theories and published data. Results of the data collection and analysis were reported at an open EMF Biological Effects meeting.

To accomplish the main objectives of this study, a simple worst-case measurement protocol was adopted. Essentially, the maximum magnetic flux time-rateof-change $(\mathrm{dB} / \mathrm{dt})$ was located by manipulating a coil around an appliance. Once the maximum $\mathrm{dB} / \mathrm{dt}$ was located, the location of the coil and the corresponding waveform were recorded. A system calibration procedure was also developed.

Initially, an acquisition system was developed for digitiring waveforms at frequencies up to $100 \mathrm{kHz}$. Later in the study, when significant high-frequency components where discovered, a second acquisition system (Figure 1) with a bandwidth of $16 \mathrm{MHz}$ was developed. The two systems were used independently to capture low and high frequency waveforms for each appliance with sample rates and digitization durations appropriately selected for each range. An independent spectrum analyzer was also used to verify the performance of the two systems.
Over the duration of the study, field characterizations were performed for eight electric shavers (including ac and battery models), five hair dryers, and four personal massage units. Several of the appliances were older models selected to correspond to those typically in use during the assumed latency period for leukemia in the population considered in the recent epidemiological study reported by Lovely et al. (1993). Significantly large ( $>10,000 \mathrm{Tesla} / \mathrm{second}$ peak to peak), high frequency (1 to $5 \mathrm{MHz}$ range) $\mathrm{dB} / \mathrm{dt}$ spikes where observed in the worst-case electric shaver (Figure 2). This places the observed fields in a region that is thought to be biologically active.

A protocol for extending this research to the measurement and modeling of hurian exposure needs to be developed. This work will form the basis for the creation of an electromagnetic field characterization laboratory with facilities for mapping the fields of personal appliances, as well as those from other sources.

\section{References}

Lovely, R. H., R. L. Buschbom, A. L. Slavich, L. E. Anderson, N. H. Hansen, and B. W. Wilson. 1993. "Adult Leukemia Risk and Personal Appliance Use: A Preliminary Study." Am. J. Epidemiol., submitted.

\section{Publication}

Wilson, B. W., N. H. Hansen, and K. C. Davis. 1993. "Magnetic-Field Flux Density and Spectral Characteristics of Motor-Driven Personal Appliances." In: proceedings of the 15th Annual Meeting of the Bioelectromagnetics Society, Los Angeles. 


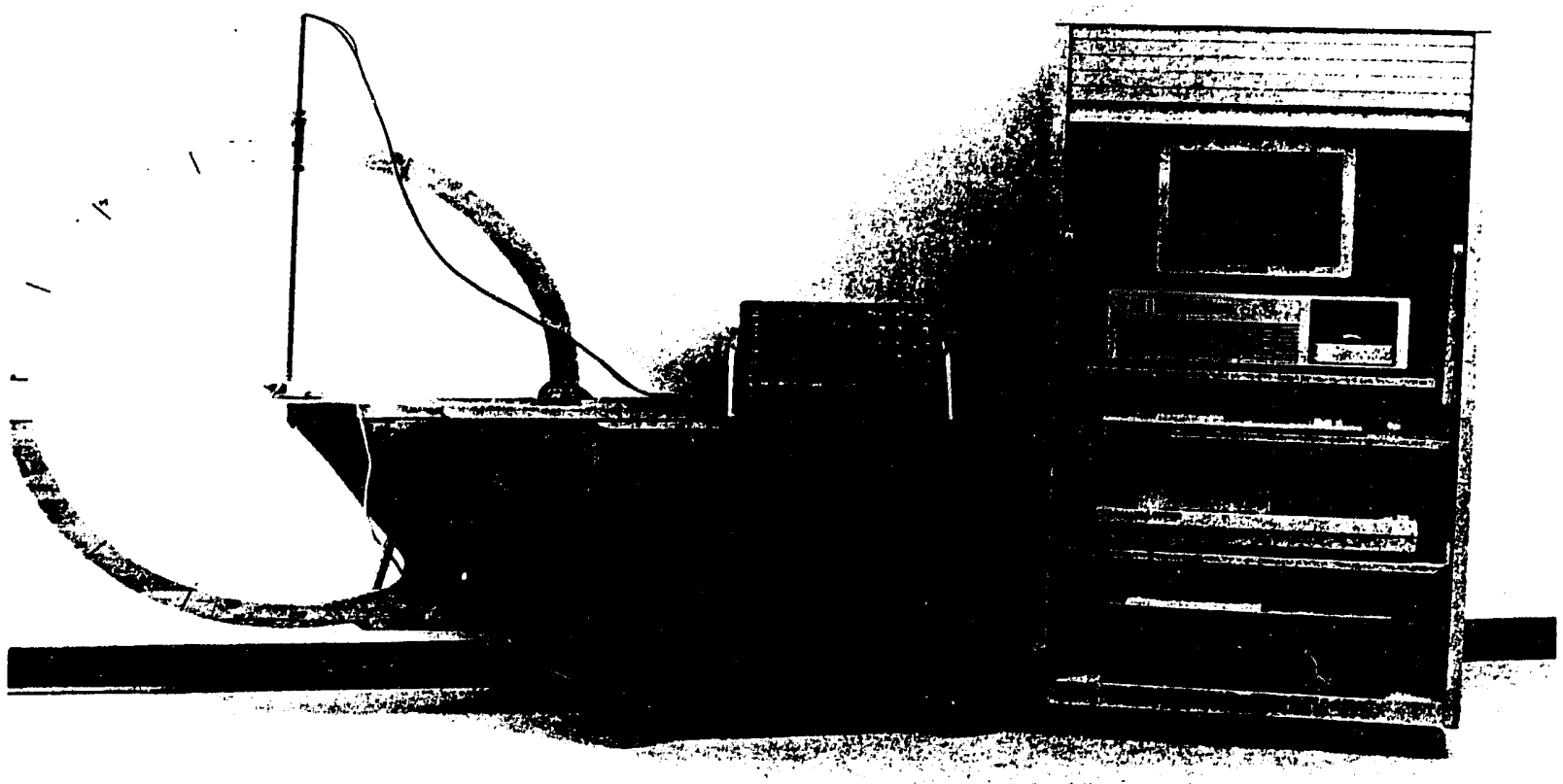

Figure 1. Electromagnetic Field Measurement System
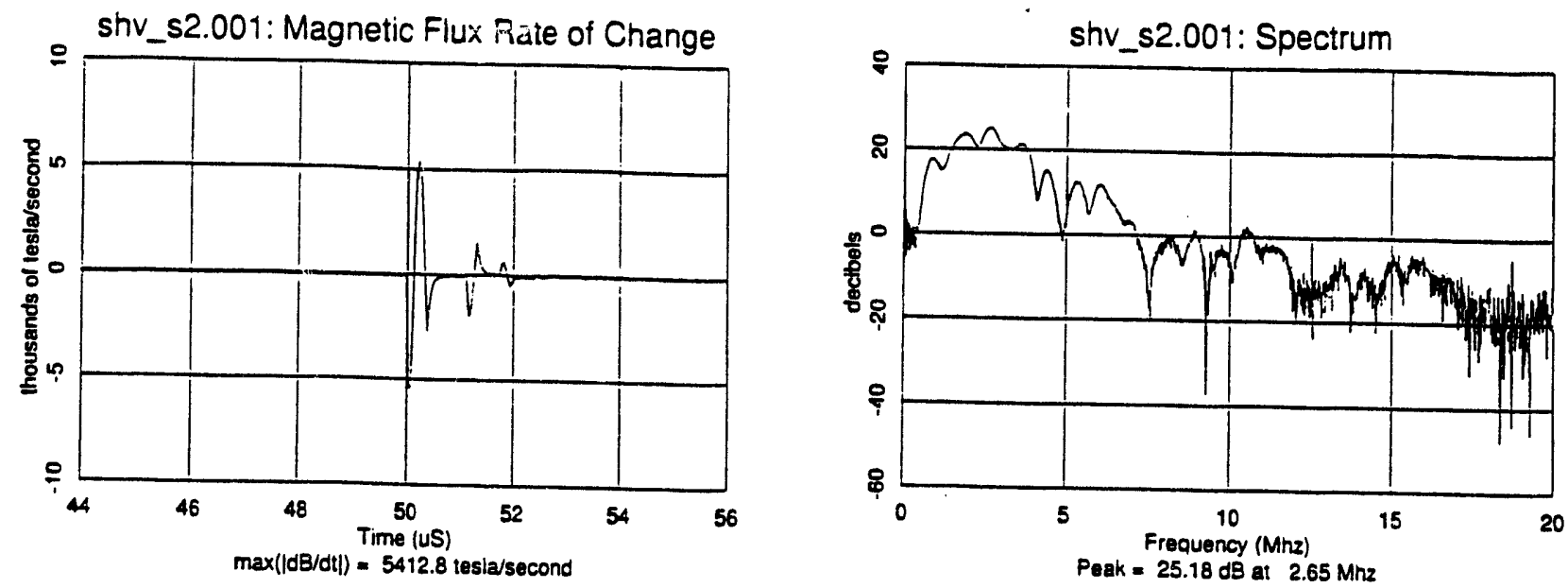

Figure 2. Electromagnetic Field Measurements for an Electric Shaver 


\title{
Environmental Catalysis
}

\author{
A. C. Hess (Theory, Modeling and Simulation)
}

The objective of this work was to gain a better understanding of the influence of surface geometry and electronic structure on the reactivity/ selectivity of heterogeneous chemical catalysts. Special emphasis was placed on identifying and subsequently understanding the surface chemistry of environmentally important catalysts capable of remediating chemical waste.

The first milestone in this project was the hiring of Dr. Roberto Nada (a postdoctoral fellow) in March 1993. During the past six months, considerable progress has been made on two different surface studies. The first centers upon the role of structural defects on $\mathrm{MgO}(001)$ surfaces and an investigation of the effect of various boundary conditions imposed during the solution of the Schrodinger equation on the predicted chemistry. The latter aspect of the study allows us to directly probe how the reactivity of the surface is affected by the effective size of the object. The second study involves a detailed comparison of experimental observations and theoretical predictions concerning the reactivity of the (0001) surface of $\mathrm{Al}_{2} \mathrm{O}_{3}$. The results of these studies are briefly outlined below.

Previously, we have investigated the absorption of molecular species such as $\mathrm{H}_{2}, \mathrm{Cl}_{2}$, and $\mathrm{H}_{2} \mathrm{O}$ (Anchell et al. 1993; McCarthy et al. 1993; Scamehorn et al. 1993) on infinitely large, atomically flat $\mathrm{MgO}$ (001) surfaces. The results of those studies demonstrated that such surfaces would physisorb but not chemisorb (or chemidissocitively adsorb) the molecular species investigated. In an attempt to understand how structural and electronic defects affect the chemistry of these systems, calculations were carried out that placed steps, edges, and kinks of various sizes on the (001) surface of $\mathrm{MgO}$. These studies have now quantitatively demonstrated that several different types of structural defects can dramatically affect the reactivity of the material. In the case of $\mathrm{H}_{2} \mathrm{O}$, for example, two independent $\mathrm{ab}$ initio methods employed in the group predict that a simple $\mathrm{Mg}_{2} \mathrm{O}_{2}$ step on the (001) surface is sufficient to make the decomposition of molecular $\mathrm{H}_{2} \mathrm{O}$ an energetically favorable process.
Recently, Dr. Wayne Goodman of Texas A\&M University, successfully synthesized a thin $\mathrm{Al}_{2} \mathrm{O}_{3}$ film consisting of a (0001) surface. In the first phase of this study we (A. C. Hess, E. Apra, and R. Nada [Nada et al. in preparation]) calculated the equilibrium structure of the $(0001)$ surface terminated by 3-coordinate $\mathrm{Al}$ species. In order to test if this was a reasonable description of the surface produced in Dr. Goodman's vacuum chamber, we each exposed the respective surfaces of the structure to the probe molecule, $\mathrm{H}_{2} \mathrm{O}$. This somewhat indirect approach is currently necessary because the experimental measurements do not yet yield the level of structural detail necessary to be compared directly with the high-level quantum mechanics calculations. Dr. Goodman observed that $\mathrm{H}_{2} \mathrm{O}$ would weakly physisorb (reversibly) on the surface he produced whereas our theoretical calculations indicated that $\mathrm{H}_{2} \mathrm{O}$ would strongly chemisorb (by approximately $28 \mathrm{kcal} / \mathrm{mol}$ ). Our current interpretation of this result is simply that whatever is in Dr. Goodman's vacuum chamber, it is not a surface consisting of 3-coordinated $\mathrm{Al}$. If it were, the water would not desorb until far higher temperatures $\left(600^{\circ} \mathrm{C}\right.$ to $\left.700^{\circ} \mathrm{C}\right)$ were reached than are currently being observed $\left(298^{\circ} \mathrm{C}\right)$ in the desorption process. As the experimental thin film surfaces are created in an oxygen rich environment, our current opinion is that surface aluminum atoms are more highly coordinated by oxygen atoms (perhaps in 4-, 5or 6-coordination). Higher coordination of these surface atoms by oxygen in the clean sample reduces the surfaces need to accumulate additional oxygen through chemidissociation or adsorption of $\mathrm{H}_{2} \mathrm{O}$. We are currently evaluating the structure and reactivity of each of the different stoichiometric and simple oxygen rich (0001) surfaces in order to experimentally/ theoretically infer the composition and structure of this surface. Even at this early stage of the project, it is quite clear that the exact nature of the surface structure and stoichiometry have pronounced effects upon the chemical reactivity of the host material.

\section{References}

Anchell, J. L., K. Morokuma, and A. C. Hess. 1993. "An Ab-Initio Molecular Orbital Comparison Study of the $\mathrm{H}_{2}$ Chemisorption on $\mathrm{MgO}$ and Lithium-doped MgO." J. Chem. Phys. 99:6004. 
McCarthy, M. I., A. C. Hess, N. M. Harrison, and V. R. Saunders. 1993. "A Study of the Energetics of the $\mathrm{Cl}_{2} / \mathrm{MgO}$ (001) Interface using Correlated Corrected Periodic HartreeFock Theory." J. Chem. Phys. 98:6387.

Nada, R., Apra, E., and A. C. Hess. "A Determination of the Structure of the Al2O3 (0001) Surface Using Correlated Corrected Periodic Hartree-Fock Theory, " in preparation.
Scamehorn, C. A., A. C. Hess, and M. I. McCarthy. 1993. "Correlation Corrected Periodic Hartree-Fock Study of the Interactions between Water and the (001) Magnesium Oxide Surface." J. Chem. Phys. 99:2786. 


\title{
Enzymatic Transformation of Inorganic Chemicals
}

\author{
H. Bolton (Environmental Sciences)
}

The objective of this investigation was to understand the mechanisms of microbial transformations of inorganic contaminants. The investigations led to establishment of a new group at PNL focused on enzymatic alteration of metals and radionuclides. For the first time, microorganisms were shown to enzymatically reduce $\mathrm{Co}, \mathrm{Cr}$, and $U$, and the mechanisms of these reactions were elucidated. This research has led to new concepts for mitigating inorganic contaminant migration in the subsurface and also for the treatment of surface waste streams containing inorganic contaminants.

The objective of this research was to investigate microbial immobilization of radionuclides and metals through redox-induced precipitation or direct enzymatic alterations in oxidation state. A number of important mobile groundwater contaminants at DOE sites have various oxidation states conducive to redox controls, including uranium, chromate, Co(III)-EDTA, and pertechnetate $\left({ }^{99} \mathrm{TcO}_{4-}\right)$. The reduced oxidation states of these metals have decreased mobility in the environment. These investigations focused on ironreducing bacteria, which can employ $\mathrm{Fe}$ (III) as a terminal electron acceptor for metabolism and reduce it to $\mathrm{Fe}$ (II), to investigate the direct enzymatic reduction of other elements.

In FY 1991 and FY 1992, the enzymatic transformation of cobalt-ethylenediaminetetraacetic acid (Co-EDTA) complex was investigated. The synthetic chelate EDTA forms water-soluble complexes with many radionuclide and metal ions. For this reason it is commonly used as a decontaminating agent in many nuclear industries and in the processing of nuclear material. The co-disposal of ${ }^{60} \mathrm{Co}$ and EDTA has resulted in the far-field migration of ${ }^{60} \mathrm{Co}$ at DOE sites. This migration of ${ }^{60} \mathrm{Co}$-EDTA is presumably because the cobalt is present as Co(III)-EDTA, which has a high stability constant $(\log K=41)$ and weakly adsorbs to sediments at neutral $\mathrm{pH}$. In comparison, the reduced Co(II)-EDTA is much less stable ( $\mathrm{Log}$ $\mathrm{K}=17$ ) and adsorbs to a greater extent. These investigations demonstrated that dissimulatory iron reducing bacteria enzymatically reduce Co(III)-EDTA to Co(II)-
EDTA under anoxic conditions. This reduction of Co(III) to Co(II) markedly increases sorption of ${ }^{60} \mathrm{Co}$ to individual phases in the environment. Similar phenomena were shown to influence the behavior of chromate and uranium.

In FY 1993, efforts were directed toward identifying and purifying the enzyme(s) that catalyze metal reduction. Completion of this task is important for a number of reasons. First, purification of the enzyme is a prerequisite to investigations of the mechanisms of electron transfer to the metal. By understanding how this enzyme operates, it will be possible to model and predict the reduction of multivalent metals in complex, heterogeneous matrices such as those found in contaminated subsurface environments. Second, enzyme purification can lead to the development of molecular probes that could be used to detect microbial populations that contain genes for metal reduction. Third, knowledge of the amino acid sequence and information from $x$-ray crystallography of the reductase enzyme could be used to develop computer models to assist in predicting the activity of a redesigned reductase. This approach may lead to increasing the selectivity of the protein for multivalent contaminant ions such as chromate, uranium, or Co(III).

The objective of this study was to partially purify the enzyme(s) and electron transport components that are involved in microbial metal reduction by DIRB strain BrY. Washed cells of BrY were ruptured under a stream of nitrogen using a French pressure cell. Unbroken cells were removed from broken cell materials by a low speed centrifugation. Membranes were separated from the soluble fraction. Fe(III)- and Co(III)-reductase activity was determined at each step of the fractionation. The results demonstrated that rupturing cells did not significantly affect the specific activity of $\mathrm{Fe}$ (III)-reductase in BrY. The results further show that the reductase activity is localized in the bacterial membrane. Separating the membranes from soluble proteins resulted in over a threefold purification of the enzyme. Soluble proteins from the periplasm and cytoplasm did not exhibit activity. Both Co(III)-EDTA and Fe(III) reductase activity were partially purified. This suggests that either the same 
enzyme is responsible for reduction of $\mathrm{Co}$ (III) and $\mathrm{Fe}(\mathrm{III})$ or that these enzymes were co-purified.

The results of these studies have led to new concepts for in situ and above-ground treatment of mixed wastes. These concepts are being scaled up and tested in prototype bioreactors for potential application to DOE site cleanup.

\section{Publications}

Gorby, Y. A., H. Bolton, Jr., and D. R. Lovley. 1993.

"Microbial Chromate Reduction and Immobilization." Pacifi. Northwest Laboratory, Richland, Washington.

Gorby, Y. A., F. Caccavo, D. B. Drecktrah, and H. Bolton, Jr. 1993. "Enzymatic Reduction of Co(III)EDTA." Pacific Northwest Laboratory, Richland, Washington. 


\title{
Epitaxial Growth and Surface Structure Determination of Novel Oxide Surfaces
}

\author{
S. A. Chambers (Materials and Interfaces) and M. A. Josowicz (Materials Science)
}

Research in the epitaxial growth, characterization, and surface chemical reactions of novel oxide surfaces relevant to environmental restoration was conducted. This approac: zombined molecular beam epitaxial growth capability, surface electron spectroscopy and diffraction, and theory to approach catalysis, chemical separation, and geochemistry in an entirely new way.

The primary goal of this work was to advance the state of the art in epitaxial growth and structure characterization of ultrathin epitaxial films, and then to use these results to grow novel oxide surfaces.

\section{Investigation of Metal Clusters in Organic} Semiconductors

Organic conjugated polymers exhibit the ability to exchange electrons with an electrode and with molecules in the solution or with neutral species in the gas phase. They can be deposited as thin films on semiconducting or conducting electrodes. Hereafter, they can be used to control the conductivity, the catalytic activity, and the hydrophobic character offering the possibility for corrosion protection in aqueous solution. In addition, conjugated polymer films deposited on inorganic semiconductors offer a possibility of forming new "organic-on-inorganic" electronic junctions with tunable characteristics. The electric characteristics of these junctions can be easily tuned by subtle chemical modifications in the polymer structure and doping level.

Based on the wealth of possibilities for electrochemical synthesis of conducting polymers, as shown by the many examples in the literature, the gorl of the present research has been to develop a general approach for characterization of the polymers during electrochemical synthesis, and then to characterize interaction phenomena taking place during subsequent exposure to neutral species. The efforts of this research during FY 1993 were focused on advancing the state of the art in electrochemical growth and characterization of the doping level in 1000 to $5000 \AA$ thick films, and then using these results to characterize gas/solid interactions between the thin polymer films and neutral species by carrying out mass (Quartz Crystal Microbalance) and work function (Kelvin Probe and Suspended Gate Field Effect Transistor) measurements. Qualitative and quantitative evaluations of charge-transfer and sorption interactions were achieved by carrying out steady-state and transient measurements.

The most important experiment in the characterization of the anodic growth of conducting polymers is to measure and then correlate the charge passing through the electrode with the mass of the deposited polymer. From this correlation, information about the propagation of the polymer lattice and the insertion of ions into the lattice of the forming polymer can be obtained. Combined electrochemical and microgravimetric measurements have been used to study the influence of the electropolymerization processes on deposition of $\mathrm{N}$-vinylcarbazole and pyrrole. The investigation of the PNVCz films applied as sensitive material indicates that the deposition mode affects the electroactivity of the layers. The correlation of the growth conditions of the polymer, specifically the water concentrations in the acetonitrile solutions containing lithium perchlorate as an electrolyte salt, with sorption and charge transfer behavior of the film during vapor exposure, clearly demonstrates the expected dependence. These results show that in the electrolyte with 2 vol\% of $\mathrm{H}_{2} \mathrm{O}$, a highly cross-linked, porous film is formed. The doping level of this film is much higher than that of the films grown from solutions with water content higher than 10 vol\%. The study of the redox switching properties of these films in the background electrolyte proves that both anions and cations participate in the charge compensation process. Furthermore, the microgravimetric data show that upon overoxidation, delamination from the substrate of the films grown from 2 vol\% water occurs. The experimental information about the nature of the polymerized PNVCz films was used to characterize the film behavior upon the exposure to propylamine vapor. A comparison of the results was 
used for comprehensive improvement of reproducibility in adsorption and desorption processes of the films.

\section{Publications}

Chambers, S. A. 1993. "Schottky Barrier Height Control at Epitaxial NiAl/GaAs(001) Interfaces by Means of Variable Bandgap Interlayers." J. Vac. Sci. Technol. A11:860.

Chambers, S. A. and T. T. Tran. 1993 "Geometric Structure at the Si/GaAs(001) Interface - The Relationship to AlAs/Si/GaAs Band Offsets." Phys. Rev. B47:13023.

Domansky, K., J. Janata, M. Josowicz, and D. Petelenz. 1993. "Present State of Fabrication of Chemically Sensitive Field Effect Transistors." Analyst 118:335-340.

Josowicz, M. 1993. "Studies of Interactions Between Conducting Polymers and Organic Vapors. Application to Chemical Sensing." In: The Electrochemical Society Proceedings, Honolulu, Hawaii.

Papez, V., P. Topart, and M. Josowicz. "Electrochemical Preparation and Study of Poly (N-Vinylcarbazole) Towards the Sensing Laser for Propylamine." J. Electroanal. Chem. Interfacial Chemistry, in press.

Topart, P., M. Josowicz. "Monitoring of Electrochemical Polymerization Process of Poly(N-Vinylcarbazole) by EQCM Method." Talanta, submitted.

Tran, T. T. and S. A. Chambers. "Interface Structure for the 1 ML Si/GaAs(001) System Studied by X-ray Photoelectron Diffraction." J. Vac. Sci. Technol. B, in press.
Yonker, B. T., S. A. Chambers et al. 1993. "Magnetic Ultrathin Films - Multilayers and Surfaces, Interfaces and Characterization." In: Materials Research Proceedings Volume 313.

\section{Presentations}

Chambers, S. A. 1993. "High-energy X-ray Photoelectron Diffraction Studies of Evolving Heteroepitaxial Interfaces." Presented at the Annual Symposium of the Canadian Association of Physics, Vancouver, British Columbia.

Chambers, S. A. 1993. "High-energy X-ray Photoelectron Diffraction Studies of Evolving Heteroepitaxial Interfaces." Presented at the First International Workshop on Photoelectron Diffraction, Gwatt, Switzerland.

Chambers, S. A. 1993. "X-ray Photoelectron Diffraction Characterization of Epitaxial Growth." Presented at the Departments of Chemistry and Physics, University of British Columbia, Vancouver, British Columbia.

Chambers, S. A. and T. T. Tran. 1993. "Interface Structure of the 1 Monolayer Si/GaAs(001)-(2x1) System by X-ray Photoelectron Diffraction." Presented at the 20th Annual Conference on the Physics and Chemistry of Semiconductor Interfaces, Williamsburg, Virginia.

Josowicz, M. 1993. "Studies of Interactions Between Conducting Polymers and Organic Vapors. Application to Chemical Sensing." Presented at the 183rd Meeting of The Electrochemical Society, Honolulu, Hawaii. 


\title{
Fisheries Consequences of Global Climate
}

\author{
M. J. Scott (Technology Policy Analysis)
}

\begin{abstract}
The objective of this work was to analyze and summarize data and literature estimates of the variation in the ocean survival of fish stocks to be used in policy models targeting on the effects of climate change on natural resources and human populations. While considerable attention has been paid to the consequences of climate change on agriculture and extensive policy modeling has been done (e.g., Adams et al. 1990; Rosenberg and Crosson 1991), relatively little has been done to summarize what the science community knows about ocean survival of fish stocks with respect to climate and the global warming issue. Since Pacific salmon stocks are sensitive to both terrestrial and marine effects of climate change, and are major predators, they are good integrators of information concerning the health of the underlying food web and other environmental conditions.
\end{abstract}

The basis for the approach used in this project was to survey the fisheries literature to develop a baseline on Pacific salmon survival parameters and then to use these parametric data to construct the simplest possible model of the salmon survival and growth process. Our initial objective was to develop a computer code that can be readily understood and easily calibrated to a number of situations where real data on salmon survival parameters are available. The current model, programmed in EXCEL 4.0, runs easily on any 486SX or later DOS-based personal computer running Windows and is readily accessible to any policy researcher familiar with spreadsheets and spreadsheet macros. Although the stock described in the prototype model is not "real," many of the climate and policy responses of the stock are real and illustrate important features of the actual climate and policy surroundings. Moreover, given a more extensive gathering and processing of field data that we describe in the accompanying paper, we believe that it will be reasonably easy to calibrate this model for real salmon stocks and to investigate the consequences of policy in the context of climate variability and change.
Because of the nonlinear nature of the climate-related impacts on fish stocks in the ocean, one natural way to model the ocean phases of salmon survival is with a chaotic system. Chaos, in the technical sense, is a state that can occur in any given system and is especially prevalent in systems that are sensitive to initial cunditions. Even very large systems such as the Northwest salmon fishery may become chaotic if at some time a very small stimulus perturbs the system. The outcome of chaotic events is unpredictable in the long term, but the application of chaos theory can provide insights into near-term effects. Chaos theory is the blend of a variety of methods useful in scrutinizing nonlinear, deterministic problems with randomness embedded in them. For example, if, through the further analysis of the fisheries data, we were able to diagnose the presence of chaos by identifying a commonly known attractor such as those of Duffing (1918), Lorenz (1963), van der Pol and van der Mark (1927), Abraham and Shaw (1985), and others, which indicate the presence of chaos, we might be able to formulate policies or strategies to mitigate the undesirable effects of the impending chaotic condition or exploit those that would be advantageous.

However, the diagnosis of chaos is not a simple task, and it may be more art than science. There is no simple measurement or calculation that can establish the existence or absence of chaos. For a system to be technically chaotic, the following specific conditions must prevail (Cambel 1993):

- The system must be nonlinear and its time series should be irregular.

- Random components must exist.

- The behavior of the system must be sensitive to initial conditions.

- The system should have strange attractors, which generally means that it will have fractal dimensions.

- In dissipative systems, the Kolmogorov entropy (a method of measuring the sensitivity of the system 
to initial conditions by calculating the rate of divergence of adjacent trajectories) should be positive.

- There are positive Lyapunov coefficients (this is another method of calculating the rate of divergence of adjacent trajectories with slightly different initial conditions).

After a simple review of our model, at least some of these conditions seem to appear. The system is nonlinear and its time series is highly irregular. We have designed random components into the model to mimic the randomness of nature. We have analyzed the system by varying the initial conditions and observed that it is sensitive to the changes. It appears that we have detected the presence of a strange attractor when we plot the same fish stock over two time periods.

\section{References}

Abraham, R., and C. Shaw. 1985. Dynamics-The Geometry of Behavior, Vols. 1-4. Ariel Press, Santa Cruz, California.
Adams, R. M., C. Rosenzweig, R. M. Peart, J. T. Ritchie, B. M. McCarl, J. D. Glyer, R. B. Curry, J. W. Jones, K. J. Boote, and L. H. Allen, Jr. 1990. "Global Climate Change and U.S. Agriculture." Nature 345:219-224.

Cambel, A. B. 1993. Applied Chaos Theory: A Paradigm for Complexity. Academic Press, Inc., 1250 Sixth Avenue, San Diego, California.

Duffing, G. 1918. Erzwungene Schwingungen bei Veranderlecher Eigenfriequenz, Braunschweig, Germany. F. Vieweg und Sohn.

Lorenz, E. 1963. "Deterministic Nomperiodic Flow." Journal of Atmospheric Sciences 357:130-141.

Rosenberg, N. J., and P. R. Crosson. 1991. "The MINK Project: A New Methodology for Identifying Regional Influences of, and Responses to, Increasing Atmospheric $\mathrm{CO}_{2}$ and Climate Change." Environmental Conservation 18(4):313322.

van der Pol, B., and J. van der Mark. 1927. "Frequency Multiplication." Nature 120:363-364. 


\title{
Fourier Transform EPR Studies of Radiation-Induced Structural Alteration of DNA
}

\author{
M. K. Bowman (Biology and Chemistry)
}

Free radicals play a central role in the damage of DNA. Ionizing radiation and even normal metabolic activity generate many free radicals that have the potential to alter DNA and cause mutation or death. Electron paramagnetic resonance (EPR) is a highly specific spectroscopic technique for identifying free radicals and for measuring the kinetics of their reactions. We used pulsed laser photolysis to generate free radicals that may be involved in damage to DNA by ionizing radiation and by chemicals. We identified and measured those free radicals using the latest fourier transform electron paramagnetic resonance techniques.

\section{Laser Generation of Free Radicals}

Light from a pulsed $\mathrm{XeCl}$ excimer laser was demonstrated to generate up to 10 micromolar of solvated electrons in two different aqueous solutions at room temperature. The solutions were alkaline phenol and alkaline tyrosine, with the best yields in the alkaline tyrosine. Lower yields of solvated electrons were generated in aqueous electrolyte solutions by illumination of an $\mathrm{Hg}$ electrode by an $\mathrm{N}_{2}$ laser. These solvated electrons reacted with cytidine directly or with $\mathrm{N}_{2} \mathrm{O}$ to produce $\mathrm{OH}$ radicals which also reacted with cytidine and other nucleic acid components.

\section{Fourier Transform Electron Paramagnetic Resonance of DNA Radicals}

Free radicals were generated in DNA and DNA components by ${ }^{60} \mathrm{Co}$ irradiation of frozen aqueous solutions. Fourier transform-electron paramagnetic resonance spectra were recorded from these radicals, which included radical anion products. The spectra were quite different even though some of thc radicals differed only in the location of a single proton. These results show a spectral sensitivity to radical structure, which can now be used to differentiate between and quantify the radical species present in irradiated DNA. In the past, radical identification has been nearly impossible because of their similar optical and conventional electron paramagnetic resonance spectra.

\section{Calculation of Spectra}

Quantum mechanical calculations using Gaussian-90 were used to model some of the free radicals expected from radiation damage to DNA. The calculated electron paramagnetic resonance parameters appear to be quite distinctive for the postulated radicals. Thus, the free radicals detected in the electron paramagnetic resonance experiments can be identified with a particular molecular structure on the basis of the quantum mechanical calculations.

Three key capabilities are now in place: 1) radical generation in aqueous solutions, 2) spectral methods to distinguish between different radicals, and 3) a means to link each radical with a particular molecular structure. It now remains to demonstrate the utility of this unique combination of techniques to address fundamental questions on the health effects of DNA damage by radiation and free radicals.

\section{Presentation}

Bowman, M. K. 1993. "Structural Applications of Pulsed EPR Spectroscopy." XV Conference on Radio and Microwave Spectroscopy, Poznan, Poland (invited). 


\title{
Fourier Transform Ion Cyclotron Resonance Mass Spectroscopy Data Acquisition and Modeling
}

\author{
R. T. Kouzes (Computing and Information Sciences)
}

The objective of this project was to develop an advanced computerized data acquisition and analysis system for Fourier Transform Ion Cyclotron Resonance Mass Spectroscopy (FTICRMS). Instruments were developed within the Molecular Science Research Center (MSRC), which were capable of making measurements on selected nuclei at a level of precision an order of magnitude better than given in the atomic mass table. Computer simulation of the instrument and electronics were carried out to allow the greatest gain in system performance.

In order to reach our goal of using FTICR-MS for making precision atomic mass measurements on a range of stable nuclei to one part in a billion, a number of simultaneous developments must occur in the data acquisitions and analysis hardware and software. The hardware needs include improved cell designs and lower noise electronics. The software needs include graphical software for modeling ion motion in order to optimize the instrument design, and analysis software tools for digital signal process of the data sets.

A postdoctoral fellow started work on this project in June 1993 to develop the understanding of the ion-ion interactions necessary for improved measurements in this system. A non-magnetic vacuum and cell system was implemented as a result of our work. Initial experiments were carried out using a Gabrielse cell, which has a more optimal configuration for improved resolution. New low noise electronics were motivated by our work which resulted in a significant improvement in signal to noise.
Computer modeling of the FTICR-MS system is a crucial aspect of accomplishing our goal in order to understand the physics of the system and to realize the most sensitive parameters to modify for improved performance. Such modeling includes magnetic and electric field calculations in three dimensions to determine the high order terms in these fields. This information is fed into ion motion simulations to study the detailed impact of non-uniformities on trapping, excitation, and detection. We have evaluated the applicability of existing computational tools for electromagnetic calculations, with the Poisson and Argus codes showing the greatest promise for usage. We have brought into operations the first of a series of ion motion simulation programs.

The resolution, and thus the mass accuracy of a FTICR-MS instrument is limited by the time that a transient is observed. Very large data sets can now be captured, but the software analysis tools must be developed to handle them. We have prepared the first version of a digital signal processing analysis code which can manipulate such large data sets. The analysis program (Digital Signal Processing [DSP]), which was developed for Sun workstations incorporates some of the capabilities for processing the large FTICR data sets. These capabilities will be extended over the next year.

We anticipate great progress over the next year in developing modeling and simulation capabilities, especially in the area of ion-ion interactions. We expect the data analysis tools to evolve significantly in capability.

\section{Presentation}

A presentation on our work was made at the Vancouver Conference on Ion Traps and will appear in the conference proceedings. 


\title{
Global Environmental Decision Making
}

\author{
S. F. Rayner (Technology Management Planning)
}

Global environmental change is attributed to two proximate human causes: land use and industrial activity. An increasingly popular perspective on industrial activity is that which adopts the metaphors of body chemistry (industrial metabolism) and of environmental studies (industrial ecology). However, systems theoretic perspectives tend to omit explicit consideration of the role of human decision making. This project examined the industrial metabolism/ecology literature and scope the potential for introducing an explicit decisionmaking focus.

At the most basic level, industrial production seeks to satisfy human needs and human wants. The magnitude of these needs and wants is affected by drivers such as population growth and changing cultural aspirations, while their realization is constrained by environmental, technological, economic, and institutional factors. The structure of demand is also influenced by government policy interventions, new knowledge, and the activities of firms.

Industry organizes to meet demand in ways that are intended, to a first approximation, to maximize profits within multiple constraints. A major organizing activity is the design of products and production processes. During design, firms balance multiple objectives of performance, cost, quality, aesthetics, and (recently) environmental impacts to create salable products. Both governmental interventions and consumer preferences influence design choices and serve as paths for introducing environmental concerns into the firm's decision-making process.

An industrial ecology perspective highlights the social value in considering the entire life cycle of material inputs and outputs. Doing so requires an understanding of the complex web of upstream and downstream relationships among the individual firms participating in the economy. Once the good passes from producer to consumer, dissipative use occurs, unless corrected by a policy intervention.
Traditionally ownership also means responsibility and liability. Thus users, companies, and other organizations often become responsible or have liability for things they do not know about. There is a mismatch between the owner's information base and his/her environmental decision-making responsibilities. The likely result is unsafe waste disposal. In other cases, such as with automobile fuel efficiency choices, the information may be adequate, but the incentive for environmentally responsible decision making is not.

Thes cases suggest a need to realign responsibilities for products during the materials life cycle, to better match information and incentives with decisionmaking power. Clearly the preferred strategy is to reduce the severity of environmental damage by product redesign or materials substitution. An alternative strategy is to institute policies or stimuli to put responsibility for high-risk products in fewer hands.

\section{Tools for Improving Industrial Decision Making}

New methods that support environmentally sensitive decision making are beginning to evolve. For industry, these tools will help at three levels: 1) they assist engineers in considering environmental criteria during product and process design; 2) they enable firms to work proactively rather then reactively with government regulators on the design of policy interventions; and 3) they help management in performing the strategic planning function by anticipating future environmental constraints, liabilities, and opportunities.

Materials balance accounting provides the means for testing the effectiveness of proposed management strategies for reducing emissions. Since the analysis is based on the principle of mass balance, strategies that do not reduce emissions, but rather shift them from one pollution pathway to another, are readily exposed.

Hazard assessments play a crucial role in the implementation of industrial ecology concepts. There are two types: 1) assessments of individual environmental problems, and 2) comparative assessments that 
describe the relative severity of different environmental hazards. Assessment of individual environmental problems is basically a scientific enterprise that focuses on the pathways through which specific chemicals or other human actions perturb the environment. Comparative hazard assessments include transscientific issues such as risk perception and management.

Product life-cycle design tools have enjoyed a modest amount of development at the conceptual level. Design tools are the mechanism for transforming broad ideas into reality. They do not exist for industrial ecology, and constitute perhaps one of the most important needs for the rapid implementation of industrial ecology concepts and practices.

Policy tradeoff analysis tools seek to balance both efficiency and equity criteria when designing policy interventions. There are several methods that are often applied to environmental policy problems:

- Benefit cost analysis works best when there is a single decision maker, no controversy, low uncertainty, and a clear understanding of all major issues. As the real context recedes from this theoretical ideal, the analyst may move from making heroic assumptions to making foolhardy ones. Risk-benefit analysis is a special case of this method that is often used in regulatory standard-setting.

- Multi-criteria analysis attempts to overcome the weaknesses of cost-benefit analysis by keeping impacts in their original units (rather than converting them to dollars), explicitly acknowledging uncertainty, and assuming that different people do not necessarily share similar preferences.

\section{Government Policy Options}

Governments' major instruments for influencing the composition of economic activity are tax and regulatory policies: corporate and personal income tax codes, excise taxes, subsidies, rate of return regulation, and standards for processes, products, equipment, and workers. Their effectiveness in specific instances has been questioned, but their capacity to alter resource allocation, as intended or otherwise, is generally acknowledged.

Information is a commodity that is especially subject to the problems of market failure. With much information, once it is produced, it is very difficult for the producer to capture its full value. Consequently, the markets for some types of information can fail to exist or can function poorly. Government can influence private sector actors to alter their behavior by improving the information available to them.

A major goal of research, development, and demonstration strategies is to reduce risk and uncertainty including technological uncertainty, cost uncertainty, demand uncertainty, institutional uncertainty, and uncertainty about external and indirect effects of the technology (e.g., health, safety, and the environment). It is often difficult, if not impossible, for an individual firm undertaking basic research to appropriate the full benefits and exclude others from using the knowledge derived from it. Consequently, private agents tend to undertake less basic research than is desirable from a society-wide perspective.

Applied research, on the other hand, sometimes suffers from a common-property problem associated with possible capture of a valuable patent by the first party to develop an innovation. In these common property situations, there is a tendency for too much research to be undertaken by multiple private parties, with the society-wide benefits from the eventual discovery being dissipated in competitive research to get patent rights.

Demonstrations can suffer from high risks associated with expensive projects that may or may not be capable of commercialization and from attendant freerider problems. Once the technology is demonstrated successfully, agents who did not share in the costs and risks of the demonstration can usually use it, at least at a license fee that may fail to capture the full social value of the new technology.

In the advanced industrialized world, the 1970s represented a decade of environmental regulation spearheaded by passage of the U.S. National Environmental Policy Act in 1970. The 1980s marked a shift in emphasis from purely command and control regulation toward fiscal incentives and market mechanisms designed to internalize environmental externalities, as embodied in the emissions trading provisions of the 1991 Clean Air Act. The 1990s may turn out to be a decade of information and research, development, and demonstration in which firms and communities become increasingly proactive in seeking environmental goods, in the absence of national governmental actions. 
On the research, development, and demonstration front, we are witnessing unprecedented levels of cooperation among industry competitors, exemplified by the U.S. motor industry's National Center for Manufacturing Science. Governments are increasingly willing to engage in cooperative research, development, and demonstration with private sector partners (e.g., through cooperative research and development agreements).

Many of the institutions and regulatory mechanisms in the industrial countries are absent or dysfunctional in developing countries. For example, the legal system in Kenya does not recognize that environmental degradation provides a basis for civil litigation. Citizens action groups, which have played a leading role in public education and environmental action in the industrialized countries, have few parallels in the developing countries. Existing research on the topic of national differences in institutional arrangements for making and implementing environment and technology policy is somewhat fragmented and mostly focused on the developed world.

The pathways for achieving sustainable development are not obvious. However, it is clear that cooperation among industrialized countries and developing and transitional countries will be crucial for moving along the pathways. 


\title{
Graphical Interface for Ocean Global Circulation Models
}

\author{
K. D. Strauss (Marine Sciences Laboratory)
}

The goal of this project was to design and prototype a single, consistent graphical user interface to simplify the use of several ocean general circulation models (OGCMs).

The first step in creating a user interface for several models is the evaluation of the models and their use. This allows us to determine what type of interface is most helpful, and what features the interface should include. The first year of LDRD funding emphasized the evaluation step. We decided on candidate models to evaluate and attempted to acquire these models. The models selected include a wide variety of types so that the resulting interface will be applicable to a large range of applications. The models chosen were: the Ocean Large Eddy Model (OLEM), the Modular Ocean Model (MOM), the Semi-spectral Primitive Equation Model (SPEM); Oberhuber's iso-pycnal model (OPYC), and the recently developed Parallel Ocean Program (POP). We were successful in getting source code for the Ocean Large Eddy Model, the Modular Ocean Model, and the Semi-spectral Primitive Equation Model. We are waiting to get the Parallel Ocean Program, which is still experimental and may not be available until late in FY 1994. We evaluated the three models by creating categories of items the interface might address, and evaluated each model to see how many items fit into which categories. The categories include: actual inputs, compilation options, implicit inputs, output routines, pre-processing routines, and format of input and output files. Based on these categories, we were able to get an idea of what a generic interface would have to look like.

The second step of creating the user interface is to actually build a strawman interface and show how it would apply to one particular model. We determined to use the Primitive Ocean Program because of our contacts at Los Alamos. We have discussed the possibility of doing an interface for the Primitive Ocean Program with scientists and programmers who use it. There seems to be strong support for the idea, however we were unable to get access to the program's code during the short time span of this project. We have not yet developed a detailed interface that would work for the Primitive Ocean Program. During FY 1994, we plan to acquire the Primitive Ocean Program, do a detailed front-end, then write a proposal to an outside funding source for the majority of the coding work.

Publications

Strauss K. D. and J. R. Bridge. 1993. "A Graphical User Interface for the Ocean Large Eddy Model." In: Proceedings of the Marine Technology Society Conference, Long Beach, California. 


\title{
Health Protection and Standards for Hazardous Chemicals
}

\author{
B. L. Harper (Health Risk Assessment)
}

This project is directed at reviewing the toxicology of a number of chemicals found on the Hanford Site. During FY 1993, existing information was evaluated for its adequacy for regulatory purposes. Four chemicals the solvents chloroform, trichloroethylene, 1,1,1-trichloroethane, and tetrachloroethylene) received the most extensive review, while cadmium, lead, and mixed chemical-radiation materials were examined in less detail. Some effort was expended to identify pertinent data bases that could be used to further examine the status of these materials, and also to identify research needs relative to regulatory standards and to human health and ecological risk assessment.

In order to identify the most important nonradioactive contaminants on the Hanford Site, several existing onsite inventory data bases were consulted. The four chlorinated solvents listed above are major contaminants at Hanford and most other DOE sites. Cadmium, lead, and mercury were also reported at most DOE sites, and chromium is also a major contaminant at Hanford. The Hanford Environmental Information System is being reviewed for adequacy of total inventory quantification of chemical and mixed waste contamination.

The four solvents plus cadmium were selected for detailed review. Reviews of pertinent literature on chloroform, trichloroethylene, trichloroethane, tetrachloroethylene, and cadmium have been summarized in draft form. In addition, the epidemiologic literature has been searched for information on simultaneous exposure of people to radiation and toxic chemicals. Chromium and mercury were postponed to future years, and lead is currently under review by the National Academy of Sciences.

The scientific basis for the regulatory standards for chloroform and trichloroethylene does not include recent information on the mechanism of their carcinogenicity. Specifically, cell proliferation rather than genotoxicity may be more important for these chemicals. Also, the metabolism of trichloroethylene in humans may not produce the same proportional amount of the reactive metabolite as the mouse, the species against which the human potency factors are scaled. Thus, the cancer potency factors for these chemicals should be reexamined and possibly recalculated. The same factors appear to be true for chloroform. However, studies have not been done for either chemical that examine the dose-response relationship between genotoxicity, metabolite formation, and hyperplasia in the target tissues and nontarget tissues.

The Reference Dose for trichloroethane is currently under review by the U.S. Environmental Protection Agency (EPA), and so does not warrant further detailed review at present.

Cadmium is regulated as an inhalation carcinogen and as an ingestion nephrotoxin, based on the scientific literature. In the absence of any new data, there does not seem to be any basis for revision of the toxicity factors or the occupational limits.

The primary noncancer concern with the four solvents is the likelihood of their interactions, mutual potentiation, and interactions with other common lifestyle substances. Since they usually occur as part of mixtures, it will be very important to examine them in light of negotiations for remediation of particular contaminated sites. In addition, a more detailed examination of the toxicity factors is needed relative to potentially sensitive subpopulations that might reasonably occur under various future Hanford Site land-use scenarios. This will be part of the focus for FY 1994.

Lead, uranium, mercury, and chromium were not reviewed during FY 1993. A primary concern with these heavy metals is, in addition to nephrotoxicity, the possibility that the standards are not adequately protective for neurotoxicity, particularly when they occur in combination with each other or with the solvents, which are also neurotoxic. This is particularly important for potentially sensitive subpopulations which might occur on the Hanford Site, and who must be considered in risk assessment and site remediation goals. This will be part of the focus for FY 1994. 
For both solvents and metals, there is a need to develop in vitro cellular screening assays that are quick and efficient and can be used to screen samples of vapors, contaminated groundwater or surface water, and contaminated soil that are collected on the Hanford Site. A potential assay has been identified through another project. In addition, other projects which are related to the toxicological aspects (such as pharmacokinetic modeling) and to risk assessment aspects are being consulted for possible interdisciplinary follow-up efforts and integration.

Discussions with DOE, DOE contractors, and regulators were begun in order to find the most efficient way to get the information into the onsite risk assessment process. Peer reviewed publication is clearly the most acceptable and this will be done in FY 1994.

Finally, additional areas of investigation were identified for FY 1994: 1) an examination of the uncertainties that are used in cancer modeling, and 2) a survey of health risk factors for the specific chemicals found on the Hanford Site, which is information essential to the risk assessments that are being performed and to the future site land uses that are being considered. 


\title{
High Field NMR and NMR Imaging
}

\author{
B. R. Stults and G. P. Drobny (Molecular Science Research)
}

\begin{abstract}
The objective of this project was to study the structural and dynamical effect resulting from the introduction of specific radiation-induced lesions into DNA oligomers. In future research, highresolution nuclear magnetic resonance methods will be used to elucidate the structural and dynamical perturbations introduced by these lesions.
\end{abstract}

High-resolution, two-dimensional nuclear magnetic resonance has long been used to determine the solution structure of biopolymers, in particular nucleic acids, proteins, and complexes of drugs. The objective of such studies was to elucidate the relationship between structure at atomic level and biological function.

This project included several phased activities to determine the

- structure and dynamics of native DNA molecules of specified sequence, as well as the structure of regulatory proteins and restriction enzymes by high-resolution, two-dimensional nuclear magnetic resonance

- structure and dynamics of regulatory proteins complexed to DNA operator sequences

- structural effect that specific DNA lesions have on these complexes.

Studies of the structure and dynamics of specific DNA oligomers were completed, samples of nuclear magnetic resonance oligomers containing lesions at defined locations were prepared, and nuclear magnetic resonance studies of the structural perturbations produced by these lesions were initiated.

Work to date has involved characterization of the structure of oligonucleotide sequences into which specific lesions will be introduced. In addition, the phosphoramidite of the lesion 5,6-dihydrothymidine has been synthesized in gram quantities. Studies of oligonucleotide structure and dynamics have been carried out by Dr. M. A. Kennedy. In particular, studies of $T_{n} A_{n}$-containing DNA oligonucleotides have been performed to determine the degree to which the adenine at the TpA junction is mobile and the degree to which any mobility is sequence dependent. Evidence for sequence specificity of the motion is derived from studies of $A_{n} T_{n}$-containing sequences, which do not display anomalous broadening of the $\mathbf{H} \mathbf{2}$ proton at the ApT junction.

Dr. Kennedy's studies have also demonstrated that in Aha III sequence specific mobility may play an important role not only in DNA-protein recognition but in enzymatic cleavage of the DNA backbone. Unusual mobility at the DNA cleavage site is extinguished by adenine methylation and methylation also abolishes the endonuclease activity of Aha III.

In what is perhaps one of the more surprising developments in high-resolution nuclear magnetic resonance methodology, Harbison and coworkers have recently demonstrated that in a system of two scalar-coupled spins that are cross relaxing to a proton bath, in the long correlation time limit cross relaxation can modulate the spectral frequencies of the coupled spins. The result is that splittings of spectral lines, commonly interpreted as due to proton scalar couplings modulated by internal molecular motions, are actually modulated by overall molecular tumbling, which drives cross relaxation. We have solved the complete Liouville equation for a system of three coupled spin $1 / 2$ nuclei, configured to simulate the H1'-H2'-H2" network in a furanose ring in DNA. Our simulations indicate that the resonance frequencies and phases of the HI' quartet are strongly perturbed by cro.s relaxation. In fact, by assuming coupling constants characteristic of a C2'-endo conformation and simply adjusting the correlation time of overall molecular tumbling from 1 to 5 nanoseconds, the $\mathrm{H1}^{\text {' multiplet }}$ can collapse to a structure that is commonly interpreted as resulting from a C2'-endo/C3'-endo equilibrium.

Considerable progress has been made in the development of an expression system for producing nuclear magnetic resonance quantities of cro/repressor protein. Our intention is to study the structure of a cro/DNA 
complex using the native OR3 operator, a palindrome operator, and an operator containing the lesion 5,6-dihydrothymidine. In collaboration with Professor Paul Hopkins, the effect of various drugs and various chemical agents (e.g., chromium, mustard) is being studied.

\section{Publications}

Kennedy, M. A., J. M. Miller, B. R. Reid, and G. P. Drobny. "Methylation Alters Base Mobility Precisely at the TpA Cleavage Site in Aha III Restriction Sequences." Biochemistry, submitted.

Kennedy, M. A., S. T. Nuutero, J. T. Davis, G. P. Drobny, and B. R. Reid. 1993. "Mobility at the TpA Cleavage Site in the T3A3-Containing Aha III and Pme I Restriction Sequences. Biochemistry 32:8022-8035.
Wang, A. C., M. A. Kennedy, B. R. Reid, and G. P. Drobny. A Solid State 2H NMR Investigation of Purine Motion in a 23 Base Pair RNA Duplex." J. Magn. Reson., in press.

Zhu, L., M. A. Kennedy, B. R. Reid, and G. P. Drobny. "The Effect of Cross Relaxation on the Spectrum of a ScalarCoupled Three-Spin System." J. Amer. Chem. Soc., to be submitted. 


\title{
Improved Analytical Ion Trapping Methods
}

\author{
R. D. Smith, A. L. Rockwood, D. W. Mitchell (Chemical Sciences)
}

\begin{abstract}
This research was directed at methods aimed at increasing the utility of ion trapping methods for analytical mass spectrometry for use in a broad range of applications. The research emphasized use of computational and experimental studies aimed at defining improved trapped ion cell geometries for mass spectrometry, methods for more efficient trapping of externally generated ions, and the use of combined electromagnetic fields to enhance performance. A new concept for single cell or particle characterization is being explored, potentially a broadly useful method for "real-time" analysis for particles having a broad range of compositions. This effort was directed at providing an initial step toward a program aimed at implementing advanced mass spectrometric methods in support of DOE environmental, waste, remediation, and health effects research.
\end{abstract}

The need for improved analytical and characterization capabilities at Hanford is en rrmous, including in situ tank characterization; groundwater monitoring; atmospheric sampling; in situ remediation support; bioremediation studies; broad laboratory-based analysis capabilities; the monitoring of waste processing, transport, and disposal; as well as health effects research.

It has been demonstrated that improved analysis/ characterization methods, and their increased utilization, can substantially decrease the cost of cleanup activities. However, current support in development of advanced methods is fragmented and is primarily driven by near-term demands.

One aim of this project is to examine a new paradigm for this process with the intent of speeding the vertical integration of improved analytical instrumentation. A second and specific aim of this project is to develop a new approach to particle characterization based upon ion trapping technology. The concept involves a method to obtain mass spectra potentially suitable for identification of single individual viruses, bacteria, or other large inorganic, organic, or biological "particles."
During the first year of this project, efforts have begun to determine appropriate conditions based upon computer modeling studies and to develop methods for the trapping and characterization of individual particles. Initial efiorts involved the evaluation of methods for the trapping of large charged particles. Simple models show that collisional processes at pressures $\left(\sim 10^{-3}\right.$ torr maximum) consistent with ion trap operation are generally insufficient to trap large particles $\left(>10^{5} \mathrm{Da}\right)$. However, initial efforts have also indicated that a combination of rapid changing of trapping electric fields combined with collisional processes (and potentially augmented by the use of broadband "RF-quadrupole cooling" techniques) would provide appropriate conditions.

These methods are anticipated to provide a basis for a greatly improved analytical methodology for characterization of a broad range of "particles," spanning the spectrum from those of biological interest (i.e., individual cells, chromosomes, viruses, etc.) to those relevant to the development of new materials. There are no methods available to do this on a practical, near real-time basis. One variation of the proposed method would involve electrospray ionization of the particles dispersed in a liquid. On the basis of our experience with electrospray ionization, we expect to transfer individual particles to the gas phase with $\sim 10^{5}$ to $10^{6}$ net excess charges. The net charge is often due to protons or cations localized at specific sites on the particle surface. One of these highly ionized particles is then trapped in either an FTICR or a quadrupole ion trap. After trapping, the particle is heated by either multiple energetic collisions due to the application of if electric fields or by laser irradiation. The coulombically unstable particle will then dissociate into numerous smaller charged fragments, perhaps as many as $10^{4}$ to $10^{5}$. A mass spectrum characteristic of the particle is then obtained by methods conventionally used with ion traps. Since the location of charge sites is known to be highly specific for large molecules (e.g., proteins and DNA), we anticipate that the dissociation spectra will be characteristic of the particle (i.e., provide a "fingerprint"). Its success depends not only on the ability to trap a particle of initially large $m / z$ (up to $10^{7}$ ), but also to efficiently trap the much lower $\mathrm{m} / \mathrm{z}$ 
dissociation products. Thus, this portion of the project depends significantly upon the effectiveness of trapping efficiency. Successful demonstration of these concepts should open numerous new research opportunities, provide a foundation to their extension into other arenas (e.g., atmospheric particulate, inorganic materials, aerosol characterization, etc.), and provide the potential for field use for environmental studies and for waste characterization. 


\title{
Infrared Atmospheric Continuum Absorption
}

\author{
S. W. Sharpe (Chemical Structure and Dynamics)
}

\begin{abstract}
The project goal was to develop experimental procedures and instrumentation to better understand continuum absorption in the infrared atmospheric window, with particular emphasis on the 8 to 14 micron spectral region. Our approach involved building a vacuum system capable of generating relatively high densities of hydrogen bonded species such as $\left(\mathrm{H}_{2} \mathrm{O}\right)_{2}$, the water dimer. These weakly bonded systems are subsequently probed via an infrared, high resolutions. tunable, diode laser spectrometer.
\end{abstract}

It has been more than half a century since $\mathbf{W}$. $M$. Elsasser (1938) first noted the continuum absorption in the earth's atmosphere. This absorption is prevalent throughout the infrared but most noticeable in two spectral regions, between 3 to 5 microns and 8 to 12 microns. The anomalous absorption was originally attributed to the pressure broadened, far wing, absorption of transitions due to water molecules. Other theories have been proposed and include absorption due to hydrated ions and absorption due to water dimers.

Despite the wealth of experimental data directly related to the infrared atmospheric continuum, there is still controversy concerning the underlying physical process. Explanations for the continuum absorption can be categorized into one of three basic theories based on hydrated ions, water dimers or pressure broadening. Currently, pressure broadening and water dimer theories (Varanasi 1988) appear to account for most of the absorption while hydrated ion theories have been almost completely dismissed.

\section{Approach}

By producing weakly bonded water clusters in a collision free environment many of the problems that plague conventional spectroscopic techniques can be avoided. In these experiments, water is heated to a known temperature (and hence known vapor pressure) and entrained in a high pressure helium carrier gas. The helium-water mixture is allowed to expand into a high vacuum chamber. During the expansion, water molecules are cooled adiabatically, and undergo complexation. Typical rotational temperatures in these types of experiments are $1 \mathrm{~K}$ to $2 \mathrm{~K}$. The clusters are then probed using a diode laser technique pioneered by this author.

Basically, the expanding effluent from a slit nozzle undergoes rapid adiabatic cooling and focusing perpendicular to the axis of expansion. Rapid cooling of internal energy relieves spectral congestion by placing the entrained clusters into their lowest rovibrational states while focusing the molecular beam removes most of the Doppler broadening inherent in standard spectroscopic tochniques. Both of these effects work in concert to increase the sensitivity of this infrared absorption technique to the theoretical limit, dictated by detector noise. Through the judicious choose of infrared diode lasers, all infrared active molecules can be studied with the notable exception of homonuclear diatomics. Current absorption detection limits are about $10^{-6}$ and correspond to about $10^{8}$ molecules/ $\mathrm{cm}^{3} /$ quantum-state. Figure 1 contains a diagram of the vacuum and laser system layout.

\section{Results}

Mass spectrometer analysis of the molecular beam indicates that water clusters are being created, including the dimer. Extensive spectroscopic searching in the 10-micron region has not yet revealed any structure which can be attributed to $\left(\mathrm{H}_{2} \mathrm{O}\right)_{2}$. Spectroscopic searching in the 6-micron region (i.e., water bending mode) has revealed sharp, well resolved spectral features which are tentatively assigned to either the water dimer or $\mathrm{Ar}^{\circ} \mathrm{H}_{2} \mathrm{O}$ complex. Recently, significant improvements have been made in our optical system and we will continue to search for water dimer in the 8 to 12 micron region. We have also observed and are in the process of analyzing the following weakly bonded species: AroH $\mathrm{H}_{2} \mathrm{O}, \mathrm{Ar} \circ \mathrm{NH}_{3}, \mathrm{CO}_{2} \circ \mathrm{C}_{2} \mathrm{H}_{6}, \mathrm{Ar} \circ \mathrm{CO}_{2}, \mathrm{CO}_{2} \circ \mathrm{N}_{2} \mathrm{O}$, $\left(\mathrm{N}_{2} \mathrm{O}\right)_{2}$, and $\mathrm{CO}^{\circ} \mathrm{N}_{2} \mathrm{O}$. These types of studies result in increased understanding of the weak, long range, intermolecular forces known as van der Waals and hydrogen bonding. 
The supersonic jet technology is also being used to investigate monomers of atmospheric interest such as the chlorine nitrate molecule. Previously, long path length, low pressure spectra of chlorine nitrate have been too congested for a complete analysis to be performed. It is our intention to probe ultra cold $\mathrm{ClONO}_{2}$ in the 12.8-micron region and perform a complete spectral analysis for the simplified spectra.

\section{Publications}

Hu, T. A., E. L. Chappell, and S. W. Sharpe. 1993.

"Infrared, Diode Laser Spectroscopy of the Ar- $\mathrm{N}_{2} \mathrm{O}$ Complex."

J. Chem. Phys. 98:6162.
Hu, T. A., E. L. Chappell, J. T. Munley, and S. W. Sharpe. 1993. "Improved Multipass Optics for Diode Laser Spectroscopy." Rev. Sci. Instrum., in press.

Hu, T. A., and S. W. Sharpe. "Diode Laser Spectroscopy of the SCO-HX Complexes." J. Chem. Phys., in preparation.

\section{References}

Elsasser, W. M. Physical Review 53:768 (1938).

Varanasi, P. 1988. "Modeling of the Atmosphere." SPIE 928, pp. 213.

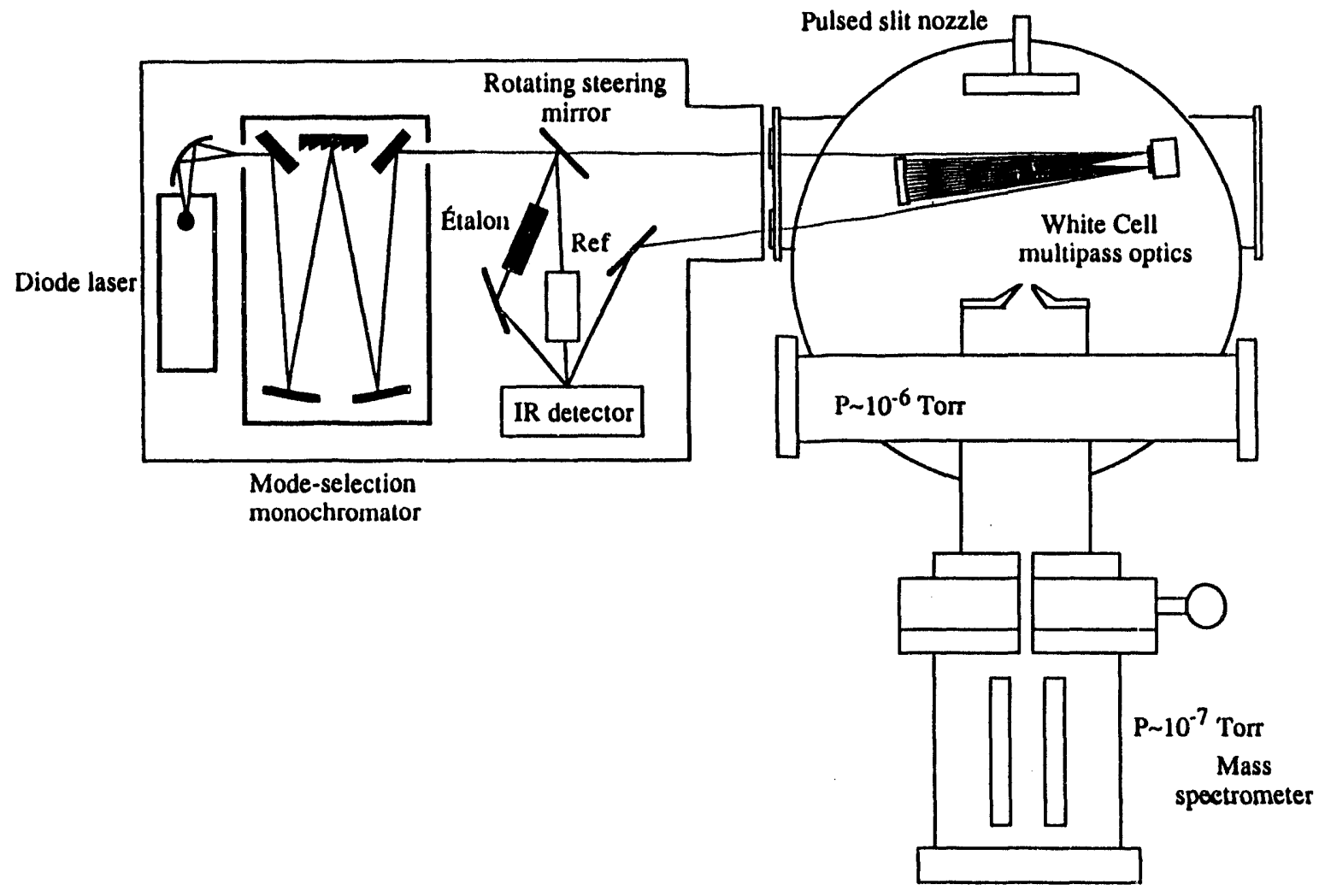

Figure 1. Diode Laser Spectrometer 


\title{
Integrated Environmental Monitoring
}

\author{
T. J. Carlson (Environmental Sciences)
}

The goal of this project was to develop and demonstrate a comprehensive, interdisciplinary environmental monitoring methodology aimed at improving the effectiveness of environmental nonitoring and restoration activities. The methodology required the comprehensive integration of monitoring regimes (i.e., in situ sampling and remote sensing), analytical practices, design methodologies, and compliance needs. The methodology tested differs from conventional approaches which involve independent planning, field implementation, and reporting of specific monitoring functions.

FY 1993 was the initial year for this project. Accomplishments include design of a framework for integration of monitoring technologies, initial development of a synthetic reality for laboratory-scale demonstration of the framework, and development of approaches to target the project to users' needs and to provide linkage with the broad scope of Hanford monitoring needs as identified through activities such as the Hanford Integrated Planning Process.

The integrated framework is an assemblage of tools and methods including optimization methods, decision analysis, error analysis, uncertainty analysis, control theory, and measurement filtering methods. The relationships between the components of the framework are shown in Figure 1.

The overall goal of framework development is to integrate monitoring technologies and to demonstrate improvements in time, cost, and decision support compared to methods currently employed in environmental monitoring programs. Key areas of development are global formulation of monitoring program objectives that can serve as the basis for decisions at all stages of monitoring, dynamic feedback mechanisms, probabilistic analysis to deal with uncertainty, and information access capabilities suitable to the various classes of monitoring information users.
The framework will be implemented utilizing commercial visualization and information management software and hardware products, available subsurface structure and process models, monitoring methodology and regulatory information data bases, and decision analysis and optimization software modules. At the close of FY 1993, evaluation of candidate technologies for each framework component were under way. Completed were preliminary specifications for user information needs and data management requirements to support framework implementation.

\section{Framework Demonstration}

The basic idea for the demonstration is to have a synthetic reality, known only to a limited number of persons, to which the integrated framework is to be applied. Data (e.g., results of pump tests, local heads, conductivities, etc.) is to be supplied from the synthetic reality as requested. The data will be corrupted with errors consistent with the measurement errors of the sensor employed. A staged test will be performed by a separate work group. This work group will not have access to the synthetic reality except from the synthetic sensor measurements. At each stage, the results (in terms of multiobjective tradeoffs and decisions made) will be compared between the integrated framework and a competing monitoring strategy. Development of elements of a prototype synthetic reality based on the 200 Area were completed in FY 1993.

Use of the synthetic reality approach will constrain demonstration costs and permit examination of a range of site configurations and problem sets while fully exercising the framework. This stage of the project promises to spin off new capabilities in addition to the framework. Some examples are

- completion of the synthetic reality will augment current geological and geophysical modeling capability

- issues related to "scale-up" of environmental monitoring from laboratory to field scales will be more clearly defined and progress made in their solution 
- methods for implementation of a probabilisticbased approach to design of monitoring programs will be developed

- an approach for exercising "synthetic sensors" under realistic constraints will be developed and demonstrated

- a laboratory-based venue for investigation of regulatory impacts on monitoring programs will be implemented
- capability for demonstrating aspects of monitoring programs to nontechnical audiences will be developed

- laboratory staff outside of the core development team will be utilized during the demonstration thereby giving them experience and insight to environmental monitoring program design and implementation.

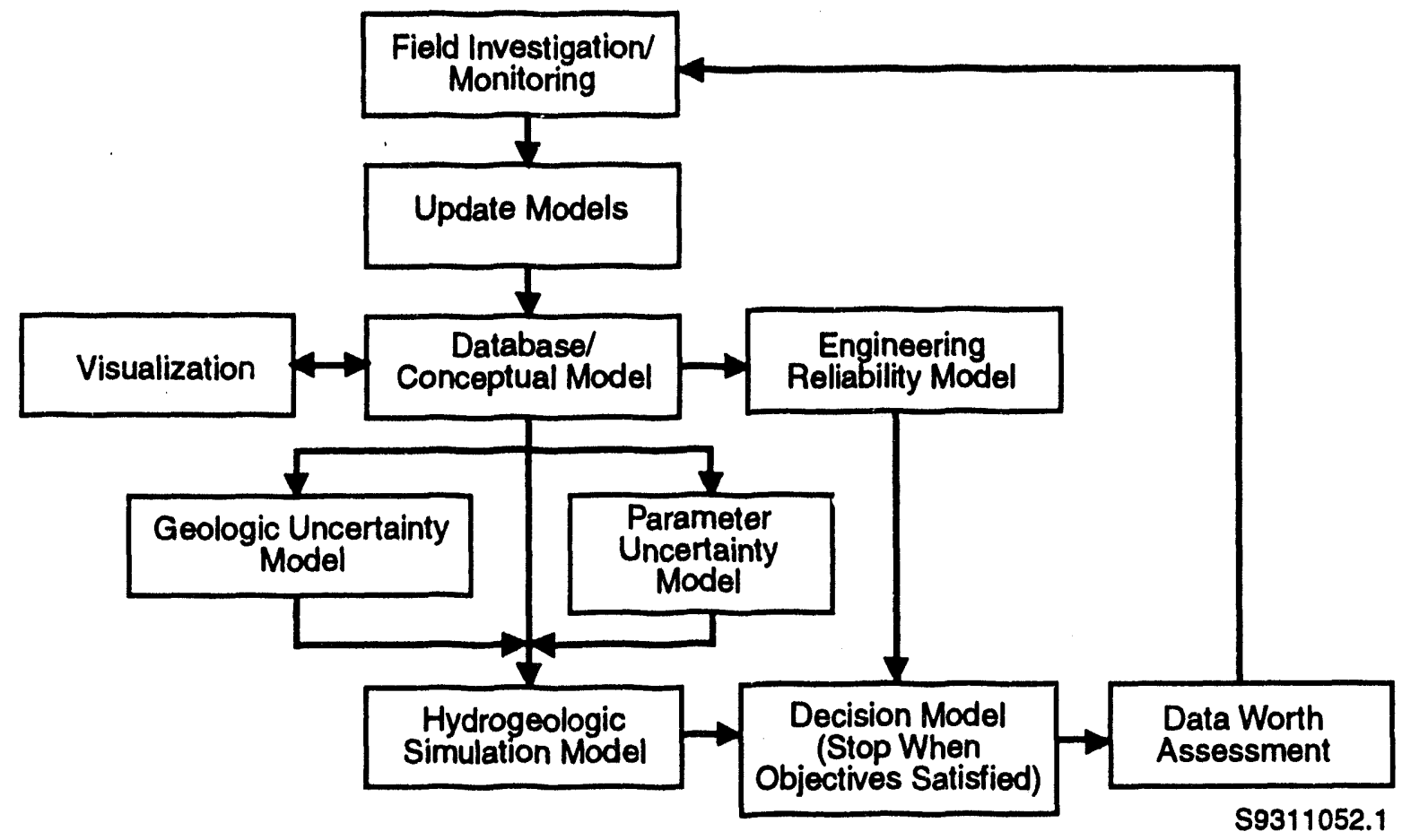

Figure 1. Integrated Framework and Relationship of Components 


\title{
Integrated Regional Climate Change
}

\author{
S. J. Ghan (Earth and Environmental Sciences)
}

The objective of this project was to develop and test procedures for predicting the regional climate and hydrologic response to climate change predictions made by global climate models.

Climate physics parameterizations have been applied to a regional atmospheric circulation model adapted to run on a workstation. Climate simulations have been conducted for the Pacific Northwest region at a resolution of $\mathbf{3 0}$ kilometers, using observed lateral boundary conditions for the period between October 1987 and October 1988. The simulated temperature, precipitation, and snow cover have been compared with observations at surface stations. All of the fields necessary for driving hydrology and ecosystem models have been archived. A parameterization of the influence of subgrid scale orography on climate has been developed for application to regional and global climate models.

During FY 1994, the subgrid orography parameterization will be applied to the regional climate model and evaluated by repeating the simulation for the Pacific Northwest. Journal articles describing the climate simulations with and without the subgrid parameterization will be prepared.

A new state-of-the-science hydrology-vegetation model, PNL-WATERSHED, has been developed. PNL-WATERSHED provides an integrated representation of hydrology-vegetation dynamics at the topographic scale described by digital elevation data. It includes a two-layer canopy model for evapotranspiration, an energy-balance model for snow accumulation and melt, a two-layer rooting zone model, and a quasi three-dimensional saturated subsurface flow model. Digital elevation data are used to model topographic controls on incoming shortwave radiation, precipitation, air temperature, and downslope water movement. The evapotranspiration component treats the effects of changes in air temperature, rainfall, cloud cover, irradiance, soil water potential, vapor pressure deficit, and wind speed.

PNL-WATERSHED has been applied to the Middle Fork Flathead River basin in Montana and compared with measured streamflow and the areal extent of snow cover. It has also been ported to a massively parallel platform to allow it to be applied to larger basins, using higher resolution data. In preparation for coupling with the regional climate model, PNL-WATERSHED was used to investigate the effects of spatial scale on the simulation of land surface fluxes and streamflow.

During FY 1994, the PNL-WATERSHED will be driven using climate model output generated both with and without subgrid scale aggregation. PNL-WATERSHED simulated streamflow and the areal extent of snow cover will then be compared with observations. The results of this research will be presented at the 1994 AGU Fall Meeting and in a jouınal article. 


\title{
Integrated Systems Ecology as an Approach for Unifying Global Change Issues
}

\author{
P. M. Irving (Environmental Sciences)
}

The goal of this project was to provide scientific support to the policy community concerning global change issues by developing a strong knowledge of the basic earth and life sciences and how they interact through analysis of the interrelationships from a unified systems point of view. Work was focused on innovative approaches to measuring, modeling, and predicting large-scale ecological change. It included developing the ability to assess the impacts and implications of local and regional landscape changes as a component of an integrated process-based earth system model. This new capability will provide a creative means for addressing long-term problems in environmental management and restoration, as well as a means for coupling environmental knowledge and concerns with regulatory, economic, and policy decision needs. The project will extend PNL's applied ecology capability beyond the realm of current programmatic expertise by permitting the acquisition and support of new staff who can integrate biogeophysical ecosystem theory for global change applications, and by cooperative interactions in the development and dissemination of knowledge concerning current state-of-the-art earth systems models.

\section{Ecological Phase Transitions}

The potential for using the concept of phase transitions to characterize ecological change was investigated. A variety of ecological phenomena at various spatio-temporal scales appears to have the properties of a phase transition, for example, the change frow snow covered to bare tundra. As a first step in this development, we plan to define the terms of reference for ecological phase transitions and to cite empirical evidence of processes that conform to these definitions. An abstract will be submitted for the annual meeting of the Ecological Society of America, and a draft manuscript is in preparation.

\section{Arid Ecosystem Modeling}

A mountain microclimate simulator, MT-CLIM, is being validated across a range of ecosystem types from desert to coastal "rain forest," as well as across geographic and topographic extremes that are responsible for vegetation regimes. Data bases for 18 longterm ecological research sites are being compiled to assess the accuracy of net solar radiation predictions based on daily maximum and minimum temperatures and precipitation. Such an extensive validation of MT-CLIM has never been undertaken, and will prove to be a valuable aspect of moving the Regional Hydrologic Ecosystem Simulation System (RHESSys) modeling efforts toward arid lands or any other biome type.

The continental-scale MT-CLIM validation is a step toward global-scale simulations of simple climatic parameters for driving ecosystem process models (maximum and minimum temperatures, radiation, precipitation).

A logic for treating the intermountain sagebrushsteppe as a mosaic of distinct, hydrologically partitioned vegetation communities was developed and some critical ecophysiological considerations for process modeling of arid ecosystems was identified. The soil water and nutrient dynamics of a forest ecosystem process model (FOREST-BGC) were modified to simulate productivity and seasonal water use patterns in Artemisia, Agropyron, and Bromus communities on DOE's Arid Land Fcology Reserve. Model relationships between soil water availability, transpiring leaf area, water use efficiency, and respiration costs of substantial below-ground productivity illustrate key ecophysiological considerations for arid land process modeling.

\section{Ecological Models for Global Change}

Literature on ecological models that might be useful for global change studies was collected and several of 
these models were examined in terms of ability to link to global climate models, usefulness for regional studies, and usefulness for answering ecological prediction and management questions at the scale of human decision making. All the models examined licked the components or scaling factors needed to answer any ecological questions on relevant spacetime scales. Only one model, IMAGE (Integrated Model to Assess the Greenhouse Effect), included socioeconomic considerations and the Edmonds/Reilly energy model. These deficiencies have been noted by others and new research efforts to develop integrated models have been initiated at several U.S. sites, notably Lawrence Livermore National Laboratory and Princeton University.

\section{Graphical Interface for Global Modeling}

A design to map the world at the country level by political boundaries was developed to allow arbitrary economic and political aggregations, and to allow mapping of vegetation within countries so that arbitrary aggregations of vegetation at regional and larger scales could be assembled. The design effort was aimed at outlining the requirements for a hierarchical spatial model structure.

\section{Parallel Computing and Workstation}

The use of transputers in a Macintosh microcomputer was investigated for development of a parallel computing system that might be used for spatiallyintensive modeling. Such a system is feasible, but the programming task is complicated and essentially requires a full-time programmer for development of useful programs. Due to the lack of software for parallel computing, a major software development effort would be entailed. The integration of various software components (e.g., graphical interface system, data base, simulation models) is a necessity and is feasible with present technology.

\section{Publications}

Kremer, R. G., and S. W. Running. 1993. "Community Type Differentiation Using NOAAAVHRR Data Within a Sagebrush-Steppe Ecosystem." Remote Sens. Enviror. 47:1-25.

Kremer, R. G., and S. W. Running. 1993. "Simulating Seasonal Hydrologic Utilization Patterns in Contrasting Semi-Arid Vegetation Communities." Ecological Modeling, submitted. 


\title{
Integration of Molecular Research with Environmental Phenomena
}

\author{
R. E. Wildung (Environmental Science Research)
}

The objective of this investigation was to conduct (through joint appointments between PNL and Washington State University) advanced research needed to link molecular-scale investigations in chemistry and microbiology to solutions of problems in environmental restoration.

There is a strong need to link capabilities being developed at the molecular level to resolution of problems being faced by the DOE in environmental restoration. Key environmental processes that must be addressed include enzyme-level phenomena governing microbial biodegradation/sequestration and geochemical reactions occurring at mineral-solution interfaces. Together with hydrologic transport these phenomena govern contaminant mobility in the subsurface and form the basis for development of new remediation concepts.

To address these needs, two new WSU/Tri-Cities positions were established in conjunction with developing capabilities at PNL and will serve as a basis for proposed new research to define contaminant behavior and develop remediation measures.

In 1991, initial scoping, planning, and research were undertaken for two joint PNL-WSU positions in organic geochemistry and microbial physiology. The Microbial Physiology/Biochemistry position was advertised in late FY 1991 and filled in FY 1992. A very strong response was received, indicating broad interest in these new concepts for development of capabilities critical to meeting DOE research needs in environmental restoration. Recruiting for the geochemistry position also resulted in a strong response, but candidates acceptable to both institutions could not be recruited. In FY 1993, the position was redefined in conjunction with the WSU Chemistry Department.

Recruiting for this new position will be completed in FY 1994 and staff will begin development of new scientific concepts.
In FY 1992, research was initiated on the degradation of synthetic chelates responsible for mobilization of metals and radionuclides in subsurface systems.

Investigations were initiated to isolate and purify the enzymes responsible for nitrilotriacetate (NTA) degradation. In addition, a graduate student was recruited and funded to clone the genes regulating the expansion of these enzymes in subsurface microorganisms.

In FY 1993, a nitrilotriacetate monooxygenase (NTAMo) was purified that has two protein components, and the $\mathrm{N}$-terminal amino acid sequence of one component was determined. A gene probe was designed using the $\mathbf{N}$-terminal amino acid sequence and it was shown that the probe is specific to only one fragment of restriction-enzyme-digested DNA from Chelatobacter strain ATCC 29600 . In addition, a summer student investigated the properties of NTAMo and found that the enzyme degraded nitrilotriacetate only when nitrilotriacetate was complexed with either $\mathrm{Mg}^{2+}, \mathrm{Mn}^{2+}, \mathrm{Ni}^{2+}, \mathrm{Co}^{2+}, \mathrm{Zn}^{2+}$, or $\mathrm{Fe}^{2+}$, but not with $\mathrm{Ca}^{2+}, \mathrm{Cu}^{2+}, \mathrm{Cr}^{2+}, \mathrm{Sn}^{2+}, \mathrm{Ba}^{2+}, \mathrm{Cd}^{2+}$, $\mathrm{Sr}^{2+}, \mathrm{Pd}^{2+}, \mathrm{Al}^{3+}, \mathrm{Cr}^{3+}, \mathrm{K}^{+}$, or $\mathrm{Na}^{+}$. An enzyme assay method was developed to detect 2,4,5-T monooxygenase and the enzyme purified. A postdoctoral fellow has been recruited to characterize the enzyme. Investigations were also initiated to characterize a water soluble blue pigment from a subsurface bacterium. The pigment properties suggest it is closely related to bacterial anthocyanins.

Several proposals were written and a National Science Foundation competitive research grant was awarded to investigate the biochemistry of 2,4,5-trichlorophenoxyacetate $(2,4,5-\mathrm{T})$ biodegradation by Pseudomonas cepacia strain AC1100.

In FY 1994, the position in microbiology will play a key role in demonstrating advanced capability being developed in the Molecular Science Research Center, Envi-onmental and Molecular Sciences Laboratory for use in bioremediation. A tetrachloro-p-hydroquinone reductive dehalogenase will be purified and labeled for 
nuclear magnetic resonance determination of structure. This will be the first application of the $750 \mathrm{MHz}$ nuclear magnetic resonance at PNL. New studies will also delineate mechanisms and isolate the key enzymes for 3-chlorobenzoate reductive dehalogenase in Desulfomonile tijiei and 2,4,5-T degradation by Pseudomonas cepacia AC1100. In addition, efforts will be undertaken to clone and sequence the gene encoding nitrilotriacetate monooxygenase.

Two papers are expected on biodegradation of nitrilotriacetate-metal complexes by a partially purified enzyme and on identification, purification, and characterization of 2,4,5-T monoxygenase.

FY 1994 accomplishments in geochemistry will depend in part on the research specialty of the individual recruited. However, the new investigator will be expected to develop joint capability in subsurface solute and colloid transport processes at multiple scales. The focus will be on 1) determining the mechanisms controlling subsurface transport of metallic hydroxide and aluminosilicate collids, and biocolloids in physically heterogeneous porous media, and 2) developing kinetic models of colloidal attachment and in deterministic modeling of experimental results. Several colloids with well-characterized surface properties, including ferric hydroxide and bacterial strains in the resting state, are being selected for study. The mineralogy, surface chemistry, and physical properties of the porous medium will be determined using, e.g., atomic force microscopy and $\mathrm{x}$-ray photoelectron spectroscopy. Laboratory column experiments will be designed to determine the rates of colloidal attachment to aluminosilicates (quariz and montmorillonite) and ferric oxide-coated quaitz. Porous medium hydraulic properties, surface structures, and the kinetic rates of attachment will be incorporated in deterministic reactive chemical transport models to aid in design of pilot-scale experiments to test the effects of hydraulic property variation on colloid transport.

\section{Publications}

Reeder, R. B., L. Xun, A. E. Plymale, and H. Bolton, Jr. 1993. "Degradation of Several Metal-Nitrilotriacetate Complexes by a Partially-Purified "Chelatobacter" Monooxygenase." Appl. \& Environ. Microbiology, submitted.

Xun, L. 1993. Archael Research. Cold Spring Harbor Laboratory Press. 


\title{
Interphase Chemistry
}

\author{
D. R. Baer (Molecular Science Research)
}

The objective of this project was to develop a new capability to examine, at the microscopic level, relationships between the structure of environmentally relevant interphases and the kinetics and thermodynamics of processes occurring in these regions, and develop the understanding required for the rational chemical and physical modification of surfaces for desired interphasial behavior.

Specific activities during FY 1993 have included designing experiments for scanning probe studies of liquid-liquid interfaces and measurement of colloid dynamics and conducting preliminary studies of important oxide, sensor, and mineral surfaces.

\section{Scanning Probe Studies at Liquid-Liquid Interfaces}

The purpose of this activity is the development of a scanning probe system to look at liquid-liquid and liquid-v/apor interfaces and to measure transport across the interfacial molecular layer.

The scanning probe microscope system available at PNL is capable of scanning tunneling and scanning force microscopy on large, flat samples. The microscope has been installed and tested for atomic resolution of standard graphite and mica samples. Atomic resolution images of these samples were obtained in both air and water. The in situ electrochemical operation of the microscope was tested on gold samples. In FY 1994 this micruscope will primarily be used to study the structure of adsorbates at liquidvapor and liquid-air interfaces. For this work a sessile drop system was assembled and filled with mercury as a model liquid. Modifications to the system are still being made to accommodate the microscope over the sessile drop and minimize mechanical vibrations.

A Langmuir-Blodgett trough is used for preparing thin films of amphiphiles by transferring the films one monolayer at a time from a liquid surface onto a solid substrate. Thus, the trough allows the controlled formation of model surfaces and chemically selective films for sensor applications. A summer student tested the trough operation by transferring fatty acid films onto glass and silicon substrates.

\section{Colloid Dynamics Project}

This project involves the measurements of fluctuations of fluorescent light to examine the mechanical and chemical interactions of micron size particles (colloids) in solution using an optical fiber.

Collaboration with R. Craig in the Applied Physics Center has resulted in the experimental design. Alterations of the laboratory in which the research will take place have just been completed. We are now beginning to assemble the experiment system and develop computer code for data collection.

Current development efforts involve both new methods of analysis and development of new materials. To assist the understanding of important sensor, mineral, and oxide interphases, some existing analysis methods have been applied to these materials. These have involved the use of scanning probe and surface analysis methods at $\mathrm{NNL}$ and the University of Washington.

\section{Publications}

Bruckner-Lea, C., J. Janata, J. Conroy, A. Pungor, and K. Caldwell. 1993. "Scanning Tunneling Microscopy on a Mercury Sessile Drop." Langmuir, accepted for publication.

Rieke, P. C., D. R. Baer, G. E. Fryxell, M. H. Engelhard, and M. S. Porter. 1993. "Beam Damage on Self-Assembled Monolaycrs." Journal of Vacuum Science and Technology A11 2292.

Rieke, P. C., B. J. Tarasevich, L. L. Wood, M. H. Engelhard, D. R. Baer, G. E. Fryxell, C. M. John, and D. A. Laken. 1993. "Patterning of Mineral Nucleation Sites on SelfAssembled Monolayers." Langmuir, submitted.

\section{Presentations}

Baer, D. R. 1993. "Environmentally Relevant Interphase Science." Presented at Rutgers University and University of Pennoylvania. 
Janata, J., C. Bruckner-Lea, J. Conroy, A. Pungor, and K. Caldwell. 1993. "Scanning Tunneling Microscopy on a Mercury Sessile Drop." Presented at the 1993 ACS meeting, Chicago Illinois. [This invited presentation was one of the featured highlights of the ACS meeting as reported in Science October 8, 1993, page 178.] 


\title{
Land Use and Agricultural Model of Global Environmental Change
}

\author{
J. F. Clarke and M. J. Scott (Technology Policy Analysis)
}

The objective of this project is to develop a model of the relationships between regional land use, agriculture, regional economic activity, and global environmental change, with an emphasis on the roles of international trade, human migration, agricultural and land management practices, technological change, and comparative economic advantage. It includes modules detailing the interaction of human population, climate, available technologies, management practices and trade, and costs of resources as they affect land use in each region.

The Land Use and Agricultural Model (LUAM) is a stand-alone model developed in parallel with the Second Generation Model (SGM) of global greenhouse gas emissions. Part or all of the Land Use and Agricultural Model will eventually be used in the Second Generation Model to provide detail in the agricultural sector.

The Land Use and Agricultural Model is designed primarily to simulate the effect of climate change or price changes on agricultural production and land use. To capture geographic changes in yield, states are grouped into one of ten farm production regions defined by the U.S. Department of Agriculture (USDA). Increased temperatures due to climate change might increase crop yield in some regions but decrease yield in others.

The Land Use and Agricultural Model is a partial equilibrium model of agricultural production with a different production function for major crops and trees in each of ten U.S. farm production regions. The Land Use and Agricultural Model's primary role is to allocate land between crops and managed forest lands in response to changes in price or climate. Land is allocated according to economic returns to land in various uses. If the price received for one crop increases relative to other crops, then economic returns for that crop will increase, and that crop's share of land will also increase.
The Land Use and Agricultural Model is designed to simulate a change in production of major crops due to changes in yield or prices or both. Climate change is modeled as a change in yield for each crop in each. farm production region. Price changes could represent an increase in the worldwide demand for food.

Land is the constraining input in the Land Use and Agricultural Model, being fixed in total supply. Within any geographic area, the largest share of land is allocated to the use with the highest return, or economic rent. The Land Use and Agricultural Model simulates changes in land rentals as prices of output change, and as yields change within each farm production region.

The Land Use and Agricultural Model has three major components: 1) a land rental module, 2) a tree module, and 3) a land allocation module. The land rental module is static because all of the crops considered here can be planted and harvested within one year. The tree module, however, is dynamic and operates on 1-year time steps from 1985 through 2030.

\section{Land Rental Module}

Production varies across farm production regions by varying technical coefficients in each production function. Inputs include prices received for each crop and yields across farm production regions. Outputs include quantity produced of each crop, and the rental value of land for each crop in each farm production region. Land rentals can be thought of as crop revenue less payments to all other inputs.

\section{Tree Module}

Trees grow in each farm production region according to a growth curve showing accumulated annual cubic feet per acre. An optimal tree lifetime is calculated based on the annualized return to land. Trees in the southeastern U.S. are harvested sooner than trees in the Pacific Northwest. 


\section{Land Allocation Module}

We are especially interested in competition for land between crops and between crop land and other land uses such as forest or biomass. Land rentals from the Land Rental Module and Tree Module are used here to allocate land within each farm production region. In the corn belt, for example, it is expected that land used for corn or soybeans will show a greater return than land used for other crops.

The Land Use and Agricultural Model is partial equilibrium in the sense that only agriculture and forest products are being modeled, and that prices of outputs and prices of inputs other than land are exogenous. Output of any given crop will increase if the price received for that crop increases, or if productivity increases due to technological change or climate change. The Land Use and Agricultural Model $\mathbf{0 . 0}$ allocates land in each of the ten farm production regions among four major crops and managed forests.

A number of cases have been run to demonstrate model response. A paper describing the model is in preparation. 


\title{
Magnetic Resonance Spectroscopy
}

\author{
H. M. Cho (Macromolecular Structure and Dynamics)
}

This project combined the development of new magnetic resonance methods with the application of established nuclear magnetic resonance and electron paramagnetic resonance techniques in a series of projects directed toward answering fundamental scientific questions arising in toxic waste management and environmental restoration and remediation.

\section{Studies of Water in Single Crystal Minerals}

A manuscript describing a proton nuclear magnetic resonance investigation of hydrous species in garnet single crystals was prepared and accepted for publication in American Mineralogist this year. The nuclear magnetic resonance data revealed that protons from water molecules trapped in the garnet lattice are grouped in closely spaced pairs, with an intrapair separation of $1.69 \AA$. The comparatively large proton-proton separation indicates that the protons do not belong to a single water molecule, but exist as separate hydrous species, e.g., $\mathrm{OH}^{-}$and $\mathrm{H}^{+}$, that have replaced an anion and cation, respectively, in the garnet lattice. The purpose of this study is to elucidate the correlation between the presence and form of water in minerals and glasses, and the macroscopic properties of the material, such as melting points, dielectric constants, rheology.

A related study on single crystals of the mineral milarite is continuing. Proton nuclear magnetic resonance results clearly indicate a macroscopic ordering of water molecules in the crystal lattice, with complicated and, as yet, poorly understood motional dynamics occurring around well-defined axes of the water molecule.

\section{Hydrogen Dynamies in Minerals}

A theoretical and experimental project to study the rates and trajectories of the motion of hydrogen in minerals was initiated this year. A postdoctoral fellow from the University of California, Santa Barbara, Dr. Robert N axwell, has been assigned to perform this work. Fully protonated and partially deuterated samples have been prepared and characterized, and a full set of variable temperature proton and deuterium nuclear magnetic resonance experiments planned to determine the parameters of motion of hydrogen atoms in this material. The goal of this study is to obtain experimental information that can guide semi-empirical quantum mechanical computations of the microscopic dynamics, atomic coordinates, and potentials present in this and related minerals. In the larger scheme, boehmite represents both a paradigm and a starting point for understanding the phenomena of water and hydrogen transport in nominally anhydrous minerals.

\section{Methodological Advances}

Much of the work in this project involves proton nuclear magnetic resonance measurements of samples containing low amounts of hydrogen. A novel variable-temperature, high-power, proton nuclear magnetic resonance probe with a low hydrogen background is being developed to facilitate these measurements. In addition, theoretical work is being performed on relaxation pathways in condensed phases, particularly external-field assisted relaxation, and on novel time-domain experiments in electron paramagnetic resonance spectroscopy and electronnuclear double resonance spectroscopy, in an effort to expand sensitivity, resolution, and information content of magnetic resonance experimeints.

\section{Presentations}

Portions of the work summarized above were reported at invited presentations in the following venues: AT\&T Bell Laboratories, Exxon Research and Engineering Laboratory, the American Chemical Society Kocky Mountain Conference, and the Russian Academy of Sciences Second Annual Workshop on Modern Developments of Electron Paramagnetic Resonance. 


\title{
Materials Surface and Interface Chemistry
}

\author{
C.H.F. Peden (Materials and Interfaces)
}

The objective of this project was to develop the experimental and theoretical research capabilities that will provide a detailed molecular-level understanding of chemical processes that occur at environmentally important surfaces and interfaces. These include processes that result in continued contamination, such as the transport of toxic species in the subsurface, as well as processes that will be used to remediate and restore the present contaminated sites. In addition, an understanding of surface chemical reactions of contaminant species is crucial to the development of selective chemical sensors for the in situ detection of these species.

Surfaces of metal oxides and carbonates are central to the majority of problems associated with environmental restoration and waste management. Not only are these materials a significant component of soil, but they also are prevalent in barrier and separation materials, catalysts, and chemical sensors. Unfortunately, very little progress has been made to understand the chemistry and physics of these surfaces compared to what is known =bout the structure and chemistry of metal and semiconductor surfaces. As such, this program is focused on developing a molecular-level understanding of the surface structure and chemical reactivity of these environmentally relevant materials. The experimental effort will primarily involve the use of ultrahigh vacuum surface science tools. A large number of ultrahigh vacuum probes have been made available as a result of the first year of this program. These include XPS ( $x$-ray photoelectron spectroscopy), LEED (low-energy electron diffraction), HREELS (high-resolutiou electron energy loss spectroscopy), AES (auger electron spectroscopy), TPD (temperature programmed desorption), SIMS (secondary ion mass spectrometıy), and FTIR (Fourier transform infrared spectroscopy). Central to this effort will be to utilize well-characterized single crystals of the oxide materials chosen for study. However, it will be more convenient in many cases to use thin-film models of the bulk materials because the application of various electron spectroscopies has been shown to be much more straightforward in this case.
Such model materials will initially be prepared in situ. Ultimately, much better characierized model thin-film materials will be provided by the Materials Synthesis Grr up in the Molecular Science Research Center.

In this past year, we have completed construction of two ultrahigh vacuum chambers containing a number of probes of surface chemical reactivity as described above. With this instrumentation, we have initiated studies of the adsorption of water and carbon tetrachloride on single crystal titania surfaces. The initial experiments are already providing important general concepts about the reactivity of these important molecules on oxide surfaces. In addition to these studies, we have developed synthetic procedures to prepare thin-film forms of titania on refractory metal surfaces that allow us to apply the full array of surface analytical techniques to understanding the surface reactivity of these materials. Studies of the reactivity of these model materials have been initiated as well, and the results will be directly comparable to those obtained on single crystal titania.

In Figures 1 and 2, we compare temperatureprogrammed desorption spectra of water $\left(\mathrm{D}_{2} \mathrm{O}\right)$ obtained by adsorption below $140 \mathrm{~K}$ either on a $\mathrm{TiO}_{2}(110)$ single crystal substrate (Figure 1), or on a model $\mathrm{TiO}_{\mathrm{x}}$ thin film grown onto a W(110) single crystal surface (Figure 2). In both figures, spectra from the bottom to the top represent increasing exposure times to water. There are some similarities between the spectra from the two systems but also at least one important differen $e$. Both surfaces show three features in the spectra below room temperature $(300 \mathrm{~K})$. However, the spectra obtained from the $\mathrm{TiO}_{2}(110)$ surface show an additional feature with a peak maximum near 310K (Figure 1) that is the first adsorption state to populate. This latter peak has been assigned to water desorbing from exposed $\mathrm{Ti}^{+4}$ sites on the $\mathrm{TiO}_{2}(110)$ surface. We believe that the lack of this peak on the model $\mathrm{TiO}_{\mathrm{x}}$ films is due to the absence of such sites on these films. We are currently performing detailed structural characterization of the thin films in order to establish this. In fact, a comparison of the lattice dimensions for $\mathrm{TiO}_{2}$ surfaces shows that there is a reasonable match between the 
structure of $\mathrm{TiO}_{2}(100)$ and the $\mathrm{W}(110)$ substrate suggesting that this surface may be favored in our case. In this regard, it is interesting to note that $\mathrm{Ti}^{+4}$ sites are not expected to be accessible for bonding to adsorbates on $\mathrm{TiO}_{2}(100)$ surfaces.

\section{Publications}

Herman, G. S., and C.H.F. Peden. "Growth of Thin Ti Films on $\mathrm{W}(110)$ and $\mathrm{O} / \mathrm{W}(110)$." J. Vac. Sci. Technol., in press.

Poden, C.H.F., and N. D. Shinn. "Oxidation of W(110): Valence-Band and W(4f) Core-Level Spectroscopy." Surf. Sci., in press.

Sault, A. G., E. P. Boespllug, and C.H.F. Peden. "MetalSupport Interactions in Hydrous Titanium Oxide-Supported Nickel Catalysts." J. Phys. Chem., in press.

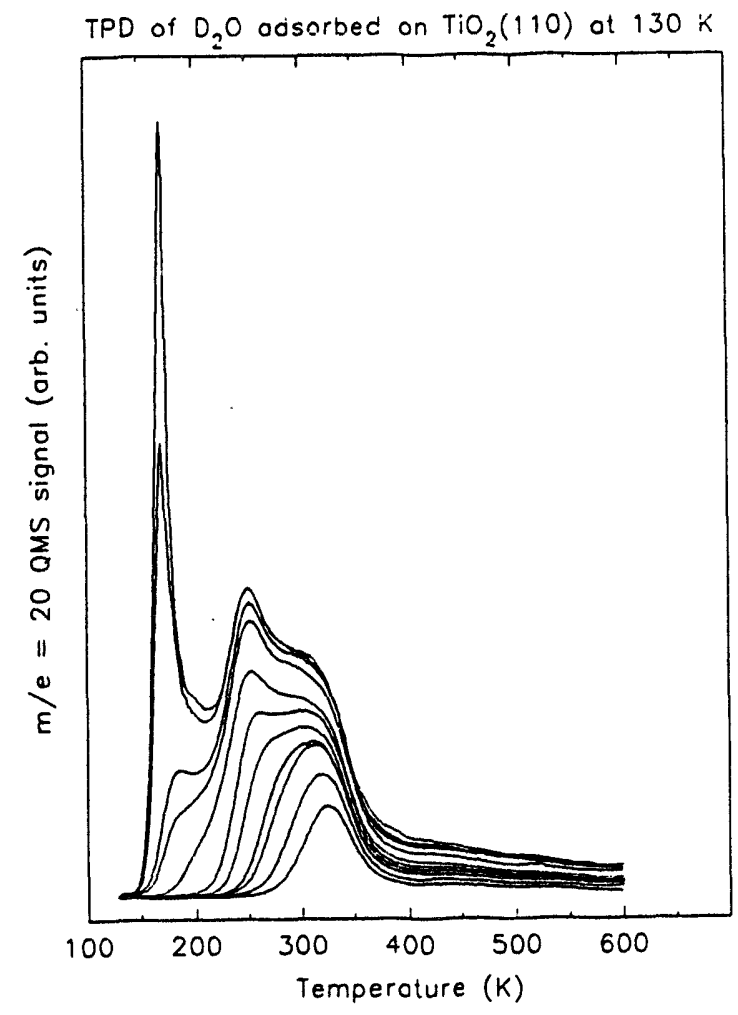

Figure 1. Mass $20\left(D_{2} O\right)$ Signal for Various Initial $D_{2} O$ Coverages on $\mathrm{TiO}_{2}(110)$ Obtained During a Linear Ramp of the Sample Temperature (TPD)
Srinivasan, S., A. K. Datye, M. J. Hampden-Smith, and C.H.F. Peden. "Interaction of Titanium Isopropoxide with Surface Hydroxyls on Silica." J. Catal., in press.

\section{Presentations}

Henderson, M. A. 1993. "Water Adsorption on $\mathrm{TiO}_{2}(110)$." Presented at the First Gordon Research Conference on Surface Chemistry, Ventura, California.

Peden, C.H.F., and N. D. Shinn. 1993. "Oxidation of W(110): Valence-Band and W(4f) Core-Level Spectroscopy." Presented at the First Gordon Research Conference on Surface Chemistry, Ventura, California.

\section{Other Accomplishments}

C.H.F. Peden (Ph.D. in Physical Chemistry) was hired at PNL from Sandia National Laboratories in Albuquerque, New Mexico, during FY 1993 to be the technical group leader for Surface Chemistry Group in the Materials and Interfaces Program of the Molecular Science Research Center.

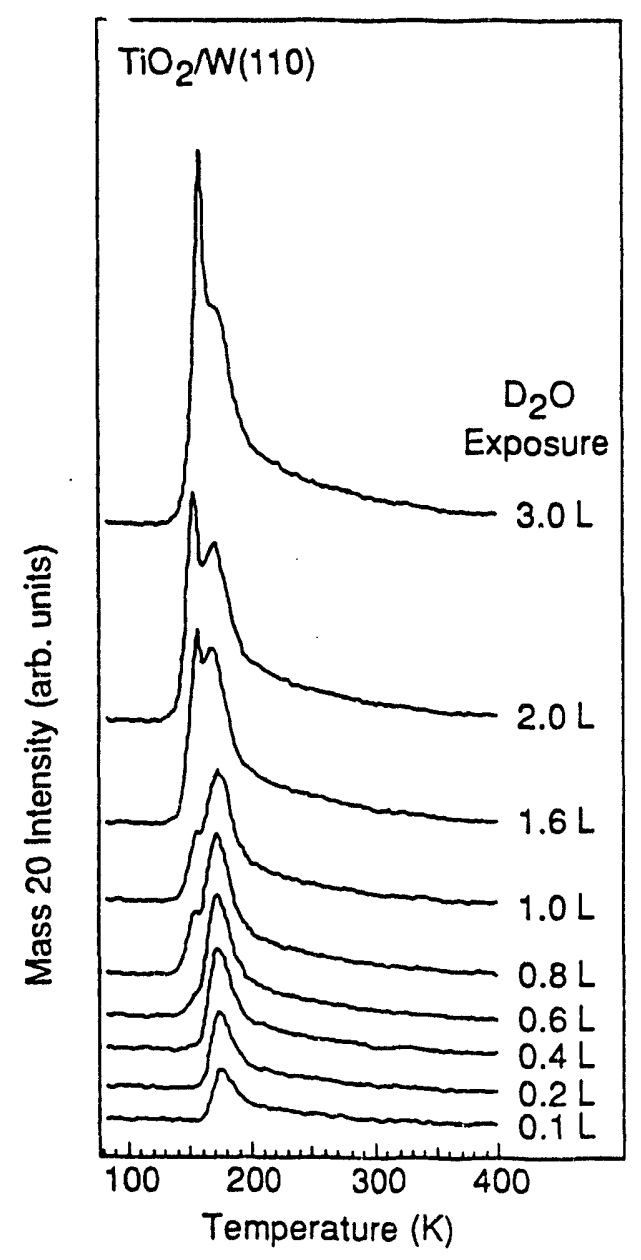

Figure 2. Mass $20\left(D_{2} O\right)$ TPD Signal for Various Initial Coverages on a Thin $\mathrm{TiO}_{2}$ Film Grown on W(110) 


\title{
Migration and Climatic Change: Events from the Dust Bowl and Their Implication for the Migration of Populations into the United States
}

\author{
J. D. Longstreth (Technology Management Planning)
}

The objective of this research was to evaluate the climatic, agricultural, and economic events associated with historical migrations from one region to another within the United States in order to determine if there are sentinel indicators which could be used as early warning signals for the conditions which induce migration. The intent was to look at several analog situations to determine if sentinel indicators were present and to evaluate whether changes in infrastructure and agricultural policy might have averted migration. The current climatic, agricultural, and econonic events occurring in agricultural and border areas of Mexico were then evaluated to determine if the appropriate configuration of events was present or was likely to occur should conditions worsen due to global climate change.

A two-pronged approach was used in this project to explore the possible implications of climate change as a driver of migration both internally within the U.S. and externally, from Mexico across the U.S. borders. The goal of the research was to build PNL's knowledge and capabilities in the social and health-related implications of climate change focusing on an issue of high potential impact: an increase in the flow of migrants into the U.S. which could have serious implications on the importation and transmission of communicable diseases.

In this study, two distinct approaches were used to explore this question. In the first approach, a detailed examination was made of the events associated with the migration of population out of the Great Plains during droughts in the 1930s. This was compared to the response of farmers to similar droughts in the 1970s where migration was not a large factor.

The second approach looked at the determinants of migration from Mexico to the U.S. focusing on the states in Mexico which send migrants into the U.S., the occupation of the migrants, the impact of droughts in Mexico on agriculture and farmers, and the resources of the Mexican government which support their agricultural industry.

Information from the two approaches was combined and the determinants of migration under these two scenarios were compared across the following five categories:

- environment, including climate and geography

- agricultural practices

- social structure

- government programs

- interventions.

\section{Environment}

The Dust Bowl area and Mexico show striking similarities in their vulnerability to drought. Both agricultural regions have semiarid to arid climates. Major droughts have afflicted both areas. For the Great Plains, major droughts have occurred in every other decade since the 1870s. Mexico historically suffers drought in at least one state every year and has regional droughts in 3 out of every 10 years. Based on physical characteristics of the land, Mexico is likely to be more susceptible to (1e adverse long-term consequences of drought bec, its rain-fed arable land slopes (making it more susceptible to soil erosion) whereas that in the Great Plains is largely level.

\section{Agricultural Practices}

In the 1930s, farmers in the Dust Bowl aggravated the effects of drought by disking, stubble burning, and overgrazing, all of which contributed to soil erosion. In addition, the introduction of mechanization favored monocropping (e.g., wheat) which depleted the soil and allowed pests to proliferate. Finally, although the bulk of the farms in the Dust Bowl were family farms, there were a sizeable number of farms operated by tenants or "suitcase" farmers who had no strong commitment to the land and thus, failed to take adequate 
protective measures to prevent soil erosion. A similar lack of erosion control practice is currently seen in Mexico, particularly in the smaller subsistence farms. In Mexico, farms fall principally into one of two categories: ejidos and neo-latifundios. The ejidos are farmed by subsistence farmers. They are numerous, but very small (on average about 30 acres), and are generally fairly marginal land. The neo-latifundios are generally considerably larger, are investor-owned and operated, and are mechanized and monocropped to produce goods for export.

\section{Economics}

In the Dust Bowl era, drought exacerbated the poor economic conditions associated with the Great Depression. At the same time, farmers grew as much wheat as they could, saturating the market and driving down the price. Similarly in Mexico, migration seems to be driven by a combination of poor economic conditions and farm failures. Large farms will likely be better able to survive droughts than the subsistence farmers in part due to irrigation systems but also because any farming support practices such as bank loans are directed at protecting the industry not the ejidos.

\section{Government Programs and Interventions}

The U.S. had few formal programs for drought relief at the start of the Dust Bowl era; however, over the course of the intervening $\mathbf{4 0}$ years, a variety of programs were added. Concomitant with the changes in support, less and less drought-associated migration out of the Great Plains was seen. Mexico's current policies are not supportive of ejido farming, particularly since farm supports (except for maize and beans) have been abolished and trading policies liberalized. However, for a brief period 10 years ago (1980-1982), Mexico did provide some government support to farmers affected by a particularly severe drought.

\section{Emigration as a Response to Drought}

In both the U.S. and Mexico, drought-associated agricultural failures were one of the drivers of migration. In the U.S., the inability of farmers to earn a living from their land exacerbated the poor economic conditions of the Great Depression. Something similar is occurring in Mexico currently. As a result of its large debt, austerity programs, population growth, and associated requirements for imported food and social development dollars, Mexico's economic conditions can clearly be characterized as depressed. This status, coupled with underinvestment in farm support programs, means that farmers see little or no opportunities in farming, and leads them to migrate to Mexican cities, or to the U.S. If increases in drought induced by global climate change were to occur, they could very well contribute to a greater degree of migration off the farms, some of which could result in a greater flow of people into the U.S.

\section{Publication}

Longstreth, J. 1993. "Health Effects of Global Warming: Problems in Assessment." Air and Waste Management Assoc $J$, in press. 


\title{
Molecular Dynamics at Solid Surface Interfaces
}

\author{
J. P. Cowin (Chemical Structure and Dynamics)
}

The objective of this project was to create methods to trace unambiguously the molecular-scale steps required for surface transformations. These reactions included those at the currently poorly understood water-solid interface, involving ionic, charge-transfer, and solvation processes. Mineral interfaces are especially important, and among the most technically difficult to study. Approaches to mineral interface studies in ultrahigh vacuum will be pioneered, including thin film mineral epitaxy.

The final year of this multiyear project has dealt primarily with demonstration of the research capabilities that have been created via the instrumentation development supported in the earlier years of the project. These "demonstrations" are in the form of publishable research, which including papers that are in preparation, will number about six. Two of the topics will be discussed below, the remainder are listed at the end of this report.

\section{Water- $\mathrm{CH}_{3} \mathrm{Cl}$ Co-adsorption on $\mathrm{Cu}(\mathbf{1 1 0})$ : Hydrophobic-Hydrophilic Effects}

Competition for surface sites between water and chlorinated hydrocarbons modifies the mobility of the latter in the environment. We used our molecular beam epitaxy and cryogenic surfaces to measure the interactions of water with a surface, with itself, and with co-adsorbed $\mathrm{CH}_{3} \mathrm{Cl}$. A copper single crystal was used because of its simple inertness (to allow the interactions between the adsorbed molecules to dominate), and because studies by other workers have shown that water adsorbs in a simple, registered, crystalline overlayer below $150 \mathrm{~K}$.

For $\mathrm{CH}_{3} \mathrm{Cl}$ adsorbed by itself, the adsorption is strongly affected by dipole-dipole interactions. The $\mathrm{Cu}(110)$ substrate binds $\mathrm{CH}_{3} \mathrm{Cl}$ with the $\mathrm{Cl}$ end down. This aligns the dipoles of the molecules (see Figure 1). We measured the binding energy as a function of coverage of $\mathrm{CH}_{3} \mathrm{Cl}$, using thermal desorption spectroscopy, and find that as the coverage increases from 0 to a close-packed monolayer, the binding energy decreases from about $11 \mathrm{kcal} / \mathrm{mole}$ to about $8 \mathrm{kcal} / \mathrm{mole}$. This decrease is generally consistent with the mutual electrostatic repulsion expected from the dipole (1.8 Debye) of the $\mathrm{CH}_{3} \mathrm{Cl}$ molecules, all co-aligned.

At 1 or less monolayer coverages, water shows attractive lateral interactions, causing the water to cluster into two-dimensional islands, leaving bare copper patches (see Figure 1). Water thermal desorption shows increasing binding energy with coverage.

Mixed layers of water and $\mathrm{CH}_{3} \mathrm{Cl}$ show that water preferentially occupies the bottom layer. For partial water monolayers, the $\mathrm{CH}_{3} \mathrm{Cl}$ first spreads out as far as possible in the bare copper areas, then will bind onto the second layer. Unexpectedly, the $\mathrm{CH}_{3} \mathrm{Cl}$ is destabilized in the bare copper patches, even at the zero $\mathrm{CH}_{3} \mathrm{Cl}$ coverage limit (see Figure 1). We have theoretically modeled this, and have concluded that this is because the water islands have dipoles which repel the $\mathrm{CH}_{3} \mathrm{Cl}$ at long range.

\section{Water and Other Molecules Adsorbed on MgO Thin Films}

Our ability to address the many surface chemistry issues related to soil and tank chemistry requires that we are able to directly study oxide surfaces, which predominate those situations. Unfortunately, single crystal forms of most of these materials are not available (like clays) or are basically impossible to maintain in a well-characterized state suitable for chemical studies with the electron or mass spectrometry-based techniques that are so well developed. We among others are applying the strategy of using single crystal conductive metal substrates to grow thin film forms of minerals epitaxially. We demonstrated this with $\mathrm{MgO}$ grown on a molybdenum surface. This sort of $\mathbf{M g O}$ growth had recently been demonstrated by Wayn- Goodman's group at Texas A\&M University; our goal was to establish the role of step defects.

Figure 2 shows the simple method of growing an epitaxial film of $\mathrm{MgO}$. In the ultrahigh vacuum system, a Mo(100) surface was cleaned and studied via electron diffraction. This clean surface was 
exposed to a small evaporator of $\mathrm{Mg}$ metal, with a simultaneous exposure to an oxygen jet. This gave $\mathrm{MgO}$ films, whose thickness we controlled from 1 to 30 atomic layers thick. Electron diffraction confirmed the single crystal nature of the growth. Using thermal desorption spectroscopy, we measured the binding of water to this surface. We reconfirmed Goodman's results showing that this surface dissociates a large fraction of a monolayer of water, and further characterized the surface as a function of annealing and film thickness. We also measured the binding energy of other molecules, including carbon tetrachloride. All suggest that this surface is a nearly perfect model of an idcal bulk $\mathrm{MgO}$ sample.

This work has shown that we can create thin-film models of minerals, which can be much better studied than their bulk crystal counterparts. This work

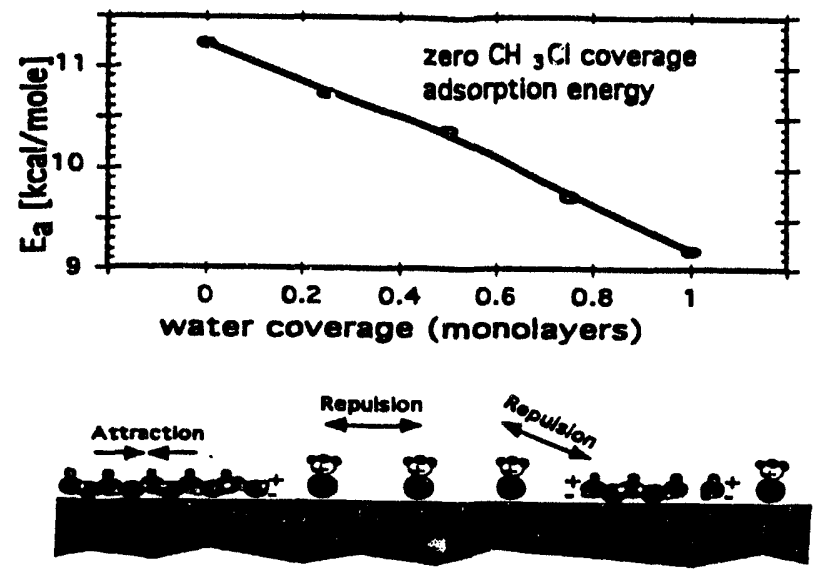

Figure 1. Mutual Interaction of Water and $\mathrm{CH}_{3} \mathrm{Cl}$ Adsorbed on a Copper Surface continues now under DOE Basic Energy Sciences programmatic research, where we very soon hope to characterize the role of steps in the $\mathrm{MgO}$ chemistry.

Other demonstration experiments include:

- photochemistry of coadsorbed water and $\mathrm{Ch}_{3} \mathrm{Cl}$ : charge transfer and radical trapping

- the structure of adsorbed water

- dipole-dipole repulsions of adsorbates: a correct simple theory.

In conclusion, we met our goal of establishing the experimental tools to recreate complex interfaces in our laboratory to study the interactions of water with other molecules on the surfaces of metals and oxides.

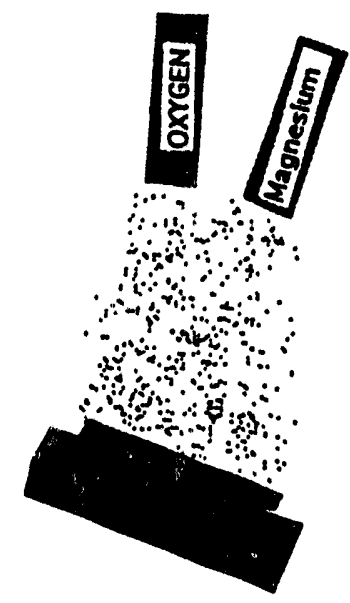

Figure 2. Growth Scheme for $\mathrm{MgO}$ on a Molydenum Surface 


\section{Multinuclear Solid-State NMR Characterization of the Early Forms of Mineralization}

P. D. Ellis (Macromolecular Structure and Dynamics)

Early phases of bone formation will be investigated by multinuclear (i.e., ${ }^{13} C,{ }^{31} P,{ }^{113} \mathrm{Cd}$ ), solid-state nuclear magnetic resonance spectroscopy. These data will prove to be essential for determining the possible role of octacalcium phosphate as a precursor phase in apatitic lattice formation. The results of this research will have significant impact on our understanding of the details of bone formation and on the synthesis and characterization of biomaterials. Likewise, this research will provide valuable insight into what processes are being altered when developing bone is exposed to radiation sources.
In FY 1993, progress was made in two areas 1) the preparation and characterization by Fourier transform infrared and powder diffraction methods of a series of apatite with varying amounts of $\mathrm{Ca}^{2+}$ being replaced by $\mathrm{Cd}^{2+}$, and 2) the utilization of the so-called "HetCor" experiment. Basically, this experiment allows one to correlate two spins by the presence (or absence) of a heteronuclear dipolar interaction. This dipolar interaction falls off as $r_{A B}{ }^{-3}$. Hence, moderate distance range structural information from the apatitic lattice will be obtained. In the case of early forms of mineralization, this leads to a significant increase in resolution. We are very anxious to apply this approach to the well-defined apatite that P. D. Ellis' group synthesized while he was at the University of South Carolina. 


\title{
PBPK-Based Breath Analysis Instrumentation Demonstration
}

\author{
K. D. Thrall (Health Physics)
}

The primary goal of this project is to combine new breath analysis instrumentation with physiologically based pharmacokinetic (PBPK) models to provide a realistic characterization of human tissue levels of volatile chemicals that pose a health concern to hazardous waste cleanup workers at Hanford. New technology using a breath inlet device connected to a mass spectrometer makes it possible to provide a real-time appraisal of components present in exhaled breath. Work on a new system is under way and is expected to provide detection of hycirocarbons and chlorohydrocarbons with a sensitivity of 10 ppb or less. Risk calculations derived from the integration of the breath analysis instrument and physiologically based pharmacokinetic modeling will be compared with results obtained by traditional techniques.

\section{Physiologically Based Pharmacokinetic Modeling}

During FY 1993, the primary goal of this project was to design a series of laboratory tests at PNL to demonstrate the capabilities of a system that combines sensitive new breath-analysis instrumentation with physiologically based pharmacokinetic models using carbon tetrachloride as the example chemical.

Physiological simulation modeling can be used to understand the fate of a chemical in the body. A physiologically based pharmacokineic model to describe the uptake, tissue distribution, metabolism, and excretion of carbon tetrachloride in experimental animals (rats and monkeys) and humans is available in the scientific literature (Paustenbach et al. 1988). The literature model, like most models, uses a series of differential equations to describe chemical distribution in the various body tissues. The equations are generally solved by the computer software program SimuSolv (DOW Chemical).
Although we were able to purchase SimuSolv and install it on a SUN SPARC 10-41 workstation, this did not occur until the end of the fiscal year. In the interim, the differential equations in the literature carbon tetrachloride physiologically based pharmacokinetic model were revised to express distribution as a rate of transfer. The model was solved using an algorithm for solving compartmental models involving recycling using matrix algebra. The drawback with using this method is that compartmental transfer must be linear, and chemical metabolism is generally a nonlinear event. However, evaluation of the literature revealed that metabolism of carbon tetrachloride is considered to be linear at low doses. Therefore, low doses were simulated to verify the physiologically based pharmacokinetic model.

Using the algorithm the model was tested, to the extent possible, against experimental animal data available in the scientific literature. Specially, the time course of the arterial blood concentration of carbon tetrachloride during a 5-hour inhalation exposure as reported by Uemitsu (1986) compared well with the model predictions (Figure 1). The model was also found to successfully predict the cumulative exhalation of both carbon tetrachloride and the metabolite carbon dioxide, the urine and fecal excretion levels, fat and liver tissue concentrations, and blood levels of rats exposed to carbon tetrachloride for 8 hours/day and for 11.5 hours/day as reported by Paustenbach et al. (1988).

In the process of extracting the existing physiologically based pharmacokinetic model, the authors' assumptions were closely evaluated for validity. A general area of concern is in the interpretation of the experimental data. In order to fully validate the physiologically based pharmacokinetic model, experimental measurement of carbon tetrachloride and metabolite concentrations in exhaled breath and in blood must be made. It was also determined that the assumption that human metabolism could be scaled 
from metabolism in rats on the basis of body weight is a major area of uncertainty. Review of the literature has identified the biological data gaps that make it difficult to extrapolate the rat physiologically based pharmacokinetic model to apply to humans. These gaps must be addressed in order to improve risk assessment.

\section{Breath-Analysis Instrumentation}

The ability to quantitatively measure, in real-time, the concentration of an organic compound in exhaled breath in the parts per billion range is possible due to new developments in instrumentation. A breathanalyzer under development uses a breath-inlet device to link a subject's exhaled breath with a mass spectrometer for a continuous analysis of specific gases; readings can be generated every 1.5 seconds.

During FY 1993, the detection limit for carbon tetrachloride in exhaled breath using the breathanalysis instrumentation and human air spiked with carbon tetrachloride postexhalation was determined to be $10 \mathrm{ppb}$. Logistic problems prevented the physical relocation of the breath-inlet device to PNL for the demonstration. Specifically, when funding was received in late FY 1993, the existing mass spectrometers at PNL had no use-time available, particularly since modification of the mass spectrometer to attach the breath-inlet device would require several days of down-time.

\section{Toxicokinetics}

Toxicokinetic studies are needed to develop the data to characterize the kinetics of chemical distribution and to describe the metabolic fate of chemicals. To fully validate the physiologically based pharmacokinetic model for carbon tetrachloride tissue distribution, it is vital to measure not only the exhalation of the parent and metabolites, but also the concentration in the transfer compartment (blood).

During FY 1993, a method was developed to measure carbon tetrachloride in blood using gas chromatography with electron capture. The quantitative detection limit was determined to be $0.3 \mathrm{ng}$ per $100 \mu \mathrm{Lblood}$.

\section{References}

Paustenbach, D. J., et al. 1988. Fundamental Appl. Toxicol. 6:484-487.

Paustenbach, D. J., et al. 1988. Toxicol. Appl. Pharmacol. 96:191-211.

Uemitsu, N., et al. 1986. Toxicol. Appl. Pharmacol. 83:20-29.

\section{Presentation}

Thrall, K. D., M. A. Cappas, and T. E. Hui. 1993. "The Role of Physiologically Based Pharmacokinetic Models in Identifying Biological Data Gaps and Reducing Uncertainties in Risk Assessment." Presented at the EPA/HERL Biological Mechanisms and Quantitative Risk Assessment Symposium, Research Triangle Park, North Carolina.

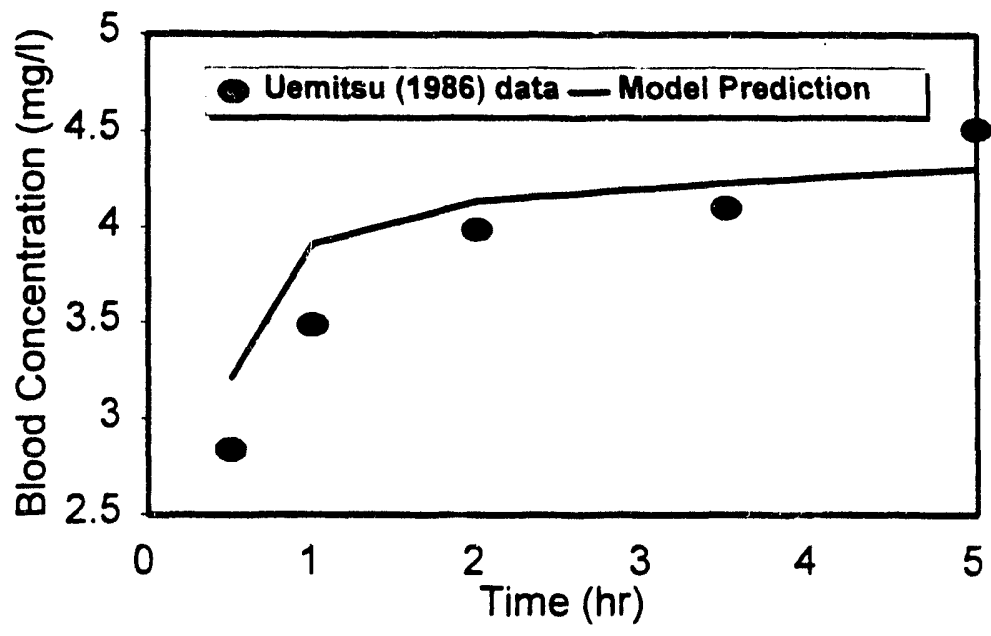

Figure 1. Comparison of Model Predictions of Carbon Tetrachloride Levels i the in the Arterial Blood of Rats Inhaling 100 ppm for 5 Hours with the Experime cal Data by Uemitsu (1986) 


\title{
Principles of Design for Global Observing Networks
}

\author{
A. M. Liebetrau (Analytic Sciences)
}

Existing global observational networks for temperature, atmospheric ozone, etc.) have emerged over time from a set of measurement stations with varying capabilities that were originally established to meet a range of diverse objectives. Consequently, existing networks have not benefited from an overall design that ensures they can produce optimal, or at least adequate, information to resolve important monitoring issues. The purpose of this research was to develop a strategy for designing surface-based networks that addresses the whole range of design, implementation, analysis, and data handling issues from a holistic point of view. The ultimate goal is to develop advanced methods for designing global-scale observing networks that provide reliable, defensible data that are adequate for resolving key scientific issues and that are not prohibitively expensive to establish and maintain.

Global observing networks (for temperature, ozone, rainfall, etc.) have emerged over time from sets of measurement stations with varying capabilities that were originally established to meet a range of diverse objectives. Consequently, existing monitoring networks have not been designed to ensure that from an overall sampling design perspective they provide optimal information. The purpose of this project has been to identify the whole range of design, implementation, analysis, and data handling issues associated with the development and maintenance of a monitoring network. This holistic perspective allows us to approach the overall problem of network design wherein each issue is treated in the context of an overall optimality criterion that is tied to the monitoring objective.

Several global scale observation ne' vorks are currently operational, and additional nutworks will be independently developed or deployed in supporting roles. The existing networks are good examples of networks that have developed as a result of fluctuations in funding or differing scientific objectives. We are left with networks made up of different measuring instruments, each with its own unique capabilities and performance characteristics. Moreover, the deployment of these instruments may or may not provide adequate spatial representation for the observed variable(s). Consequently, even with the large quantities of data existing networks produce, scientists are still not armed to answer important questions about global trends (such as, for example, ozone depletion or global warming).

Eight major facets of network design have been identified through this research, each of which must be considered in designing an "optimal" monitoring network. These facets are 1) identification of end users, 2) network design, 3) instrumentation, 4) sampling protocols, 5) data management, 6) data verification and analysis, 7) data fusion and interpretation, and 8) network modifications. Each of these factors will affect how a network is designed. Moreover, the relative importance of these eight factors will be governed to varying degrees by external influences. In designing a global observing network that provides coherent and defensible results, each of these factors must be addressed in the context of the overall sampling objective.

The major activity of the current funding period was to identify and study the eight aspects of network design identified above. Results are presented in a draft white paper, which is now ready for peer review. The paper shows some of the work that has gone into the identification and explanation of network design, the application of the ideas under various example extraneous factors. The paper also contains basic information on spatial statistics together with a bibliographic summary.

Although the paper is currently only at the draft stage, it does serve its primary purpose: To identify the primary contributing factors in designing a coherent and consistent monitoring network, and further to focus future detailed research into various aspects of these factors. It is planned to complete the white paper during FY 1994 and to proceed in the indicated research directions. Results will be presented to the scientific community in the form of presentations, technical reports, and refereed papers. 


\section{Publications}

Hardin, J. W., and R. B. Upson. "Estimation of the Global Average Temperature with Optimally Weighted Point Gauges." Journal of Geophysical Research-Atmospherics, in press.
Hardin, J. W., and R. B. Upson. "Reconstruction of the Earth's surface Temperature Field from Point Measurements: A Spectral Approach." Journal of Geophysical ResearchAtmospherics, in preparation. 


\title{
Protein Structure Analysis
}

\author{
R. J. Douthart (Life Sciences)
}

The objective of this project was to investigate the relationships between primary-secondary and tertiary protein structure using the sequence attributes method with Keystone parallel processor and neural network approaches.

A collaborative team effort involving Dr. Keith Dunker (Washington State University) and Dr. Richard Douthart (PNL) has been highly successful. The structural attributes method previously developed provides an analysis of the effectiveness of various algorithms in predicting secondary structure. A given scheme is examined for predictive and discriminative capability for types of secondary structure using Bayesian statistical methods.

Previous work done by Rob Miller was a logical extension of the method examining the Dihedral $\Phi, \psi$ angles of the peptide bond. These angles have been shown to take on correlated sets of values depending on secondary structure.

Pattern recognition algorithms were used to objectively examine these relationships. To our surprise the scattergrams (Ramashadren diagrams) that divide $\Phi, \psi$ configuration space into helix and sheet domains is further subdivided into statistically significant subregions within the large alpha and beta domains by the maximum-minimum pattern recognition algorithm.

In FY 1993, these cluster centers were studied further by creating connected three-dimensional small polypeptide backbones and exploring common motifs by extended homology modeling and three-dimensional conformation superposition. Our studies indicate that repeated substructures or structural motifs somewhat independent of amino acid identity can be ascertained.

This representation is significantly different than standard secondary structure descriptions such as helix turn or sheet, and has the added feature of being independent of sequence size and classical domain boundaries.
The proteins crythrocruorin (all $\alpha$ ) and actinoxanthin (all $\beta$ ) and Ribonuclease A (mixed) were examined for correlations between conformational clusters and reported secondary structure.

Earlier studies indicated that $\alpha$ structure gave the strongest attribute signals so as expected the sharpest cluster profiles and correlations with previous identified secondary structure regions were obtained with the protein erythrocruorin.

Profiles obtained for the other two proteins gave reasonable correlation with reported secondary structure regions. This comparison, though necessary, is deceiving since secondary structure identification by attributes is on the average only about $50 \%$ to $60 \%$ predictive and should not be taken as a standard.

The profiles are unique and revealing of many structural subentities which are discussed in detail in our recent publications. Of spe sial interest is the fragment VNTFV (letters identify amino acids) that appears at two locations in IR erythrocruorin in completely different conformations. This fragment was correctly placed in two different cunformational clusters by our methods. Standard algorithms based upon sequence identity always place the same sequence in the same structural class.

\section{Publications}

Miller, R. T., R. J. Douthart, and A. K. Dunker. 1993. "An Alphabet of Amino Acid Conformations in Protein." In: Proceedings of the Hawaii International Conference on System Sciences 26:689.

Miller, R. T., R. J. Douthart, and A. K. Dunker. 1993. "Identifying Repeated Structural Elements in Proteins." Froceeding of the Twenty-Seventh Hawaii Conference on ystem Science, in press.

Miller, R. T., R. J. Douthart, and A. K. Dunker. 1993. "Learning an Objective Alphabet of Amino Acid Conformations in Protein." Techniques in Protein Chemistry 4:541. 


\section{Presentations}

Miller, R. T., R. J. Douthart, and A. K. Dunker. 1993.

"Conformational Cluster Centers and Repeated Substructures in Proteins." Presented at the Ma molecules, Genes, and

Computers, Chapter III, Waterville Valley, New Hampshire.

Miller, R. T., K. J. Douthart, and A. K. Dunker. 1993.

"Identifying Repeated Structures in Proteins." Presented at the Seventh Annual Meeting of the Protein Society, San Diego, California.
Miller, R. T., R. J. Douthart, and A. K. Dunker. 1993. "A Set of Distinguishable Amino Acid Conformations in Proteins." Presented at the Biophysical Society Meeting. 


\title{
Radiative Forcing by Anthropogenic Sulfate Aerosols
}

\author{
C. M. Berkowitz (Atmospheric Sciences)
}

\begin{abstract}
This project was directed toward evaluating the role of anthropogenic sulfate aerosols in determining the radiative forcing of the climate. The focus is on using satellite data to evaluate model performance. Scientific objectives include an evaluation of a model that could serve as a platform for other numerical descriptions of aerosol processes in the atmosphere. The Global Chemistry Model will be the tool for this work. Aerosol formation will be simulated using a simple gas and cloud chemistry mechanism, and the total aerosol burden over the troposphere will then be used to evaluate the short-wave scattering in space.
\end{abstract}

It has been postulated that scattering of sunlight by aerosols can significantly reduce the amount of solar energy absorbed by the climate system. One group estimated that the reduction in the annual average insolation could be as much as $7 \%$ over the eastern United States. Others have used climatological values of aerosol mass loading, cloud cover, and albedo to suggest that radiative cooling associated with the scattering of solar radiation by anthropogenic aerosols is comparable to the radiative warming associated with absorption of terrestial radiation by radiatively active gases (the "greenhouse" effect).

Aerosol measurement programs alone cannot provide all the information needed to evaluate the radiative forcing due to anthropogenic aerosols. Surface-based aerosol monitoring programs do not provide global coverage from which to evaluate these postuluted effects. Although satellites also provide global aerosol measurements, estimates of scattering by aerosols are restricted to the marine atmosphere and many questions related to source attribution and anthropogenic versus natural contributions cannot be addressed. Thus, comprehensive global-scale aerosol models, properly validated against surface-based and satellite measurements, are a fundamental tool for evaluating the impacts of aerosols on the planetary radiation balance.
Models with various degrees of detail have been used to evaluate the radiative forcing. Early investigators used a back-of-the-envelope approach. More recently, use has been made of a three-dimensional meteorological/chemical model to evaluate the global distribution of aerosol forcing. Using a statistical description for removal by precipitation, monthly mean winds, and fixed deposition velocities, scientists estimated peak aerosol optical depths over North America of $\tau_{\alpha} \approx 0.12$ at visible wavelengths. Geographical distributions of aerosols agreed well with earlier measurements of turbidity. It was estimated that the uncertainty of calculations of global radiative forcing by aerosols was a factor of two. An examination of this early modeling work found, by accounting for the variability of optical depth with wavelength, an aerosol radiative forcing that is a factor of two smaller than that of previous studies $\left(0.3\right.$ versus $\left.0.6 \mathrm{~W} \mathrm{~m}^{-2}\right)$.

Confidence bounds for models describing the spatial, temporal and physicochemical variation of aerosols must be evaluated before the models can be used for either scientific or policy related purposes. Here we present $\mathrm{f}$ reliminary results of such an evaluation.

\section{Approach}

Analyzed meteorological fields from the European Centre for Medium-Range Weather Forecasts (ECMWF) are used to drive a modified version of the PNL Global Chemistry Model (Luecken et al. 1991) applied to the atmospheric sulfur cycle. The resulting sulfate fields are used to calculate aerosol optical depths, which in turn are compared to estimates of aerosol optical depth based on satellite observations from the NOAA-9 Advanced Very High Resolution Radiometer (AVHRR) (Wagener et al. 1994).

\section{Simulated Optical Depth}

The Global Chemistry Model simulations provide the spatial and temporal variations in the concentration of sulfate aerosol, $\mathrm{C}_{\mathrm{SO}_{4}^{2-}}\left(\mathrm{g} \mathrm{m}^{-3}\right)$, for October 1986 , over a domain encompassing $140^{\circ} \mathrm{W}$ to $62^{\circ} \mathrm{E}$, and from $12^{\circ} \mathrm{N}$ to $81^{\circ} \mathrm{N}$. The vertical range extends to $\mathrm{p} \geq$ 
$100 \mathrm{hPa}$. We use a simple algorithm to calculate the aerosol optical depth, $\tau_{\alpha}$, as a function of relative humidity, RH, and height, $z$,

$$
\tau_{\alpha}=\int f(R H) \sigma_{d y} C_{s O_{4}} d z .
$$

where the dry aerosol specific extinction cross section, $\sigma_{\mathrm{dry}}$, is calculated from the Mie theory for pure ammonium sulfate arosols. The refractive index of ammonium sulfate is 1.53 . We assume a particle radius of $0.15 \mu \mathrm{m}$ and calculate the specific extinction at a wavelength of $0.65 \mu \mathrm{m}$ (which corresponds to the mean wavelength of the satellite channel used to measure observed optical depth). This calculation yields a value of $\sigma_{\mathrm{dry}}=5.3 \mathrm{~m}^{2} \mathrm{~g}^{-1} \mathrm{dry}$ sulfate. The correction term $f(R H)$ is necessary to account for the growth of soluble aerosols through the absorption of water vapor. We have calculated the correction term based on field measurements for relative humidity ranging from $40 \%$ to $90 \%$. The correction term ranges from unity for $\mathrm{RH}$ below $50 \%$ to 3 for $\mathrm{RH}$ above $90 \%$. Relative humidity is taken from the ECMWF analysis.

\section{Observed Optical Depth}

The observed clear-sky aerosol optical depth is derived from NOAA-9 AVHRR radiance measurements obtained from a polar sun-synchronous orbit that crosses the equator at approximately 14:30 LST. Using the single-scattering approximation, the aerosol optical depth is expressed in terms of the aerosol single-scattering albedo, the aerosol-scattering phase function, and the solar radiation scatterud toward the satellite by aerosols. The radiation scattered by aerosols is determined as the difference between the radiance observed by the satellite and the radiation scattered by the surface and by air molecules, with the latter parameterized using the classical theories of Fresnel and Rayleigh. The method applies only to clear sky (low optical depth) scenes over the oceans (known surface reflectance and spatial uniformity). Data are subject to a number of checks to ensure suitability, including tests for the presence of high (optically thin) clouds and sunglint, both of which would corrupt the calculation of the aerosol optical depth. Details are provided by Wagener et al. (1994).

\section{Results}

Figure 1 shows the observed and simulated aerosol optical depth for October 23, 1986. Points that satisfy none of the criteria for measuring optical depth are unshaded in both plots. Thus, all land is unshaded, and much $c_{1}$ the oceans is unshaded because of obscuration by cloud cover. Several large patches remain where optical depth can be measured for this particular date.

The largest observed optical depths are in the western North Atlantic, offshore from the industrial sulfur sources in the eastern United States. Optical depths approach unity in a few pixels. Observed optical depths are substantially lower across most of the North Atlantic Ocean, with values of typically 0.1 . However, much larger values (exceeding 0.3 ) are observed near the west coast of Africa, presumably due to westward transport of dust from the Sahara.

The simulated optical depth exhibits a similar pattern offshore from the east coast of North America. Values approach 0.7 in a few pixels. The simulated optical depth decreases to about 0.05 in the central and eastern North Atlantic, with lower values in the subtropics than in mid-latitudes. Because the model treats only sulfur species, it does not simulate the high optical loading observed off the west coast of Africa. The simulated optical depth appears to be generally lower than that observed.

The spatial structure of the bias can be assessed by examining the ratio of the simulated optical depth to that observed. The bottom panel of Figure 1 shows the $\log _{10}$ of the ratio. A log scale is used because it provides a uniform resolution of positive and negative fractional errors.

The simulated optical depth is somewhat greater than the observed loading over several patches in the western North Atlantic. It is somewhat lower in nearby pixels, so that the errors in local magnitude might simply be due to small errors in position. The bias in the large patches near the Azores and adjacent to western Europe is rather uniform, with the simulated optical loading about $50 \%$ to $70 \%$ of that observed. The bias is stronger in the subtropics, both immediately west and far to the west of Africa, where the simulated optical depth is roughly $20 \%$ of that observed.

To provide more comprehensive spatial coverage in our evaluation, we have collected values at the first points during the period between October 23 and October 30 where aerosol optical depth at that point 
could be measured. Increasing coverage over the model domain was provided as additional points from each day of satellite observations were used to develop Figure 2. This method of filling in a relatively sparse data set was selected over schemes using time averaging because the latter would mask the time-resolving capability of the model. By presenting the data this way, a comparison between satellite and modeled aerosol optical depth can be done over most of the North Atlantic south of $50^{\circ} \mathrm{N}$ during this period, with exceptions in the Gulf of Mexico and scattered patches across the central Atlantic, for which there are no satellite observations of aerosol optical depth.

The patterns of optical depth evident in Figure 1 are repeated for the same points in Figure 2. The spatial extent of Saharan dust is more evident in the composite, as is the distribution of aercsol in the western North Atlantic. The simulated aerosol extends farther offshore from the east coast of North America than the observed aerosol, except for a patch to the southeast of Newfoundland that is not that extensive in the simulated values. The observed optical depth exhibits more spatial structure than the simulated values over the central and subtropical Atlantic; the simulated optical depths are systematically too low.

The $\log _{10}$ of the optical depth ratio (simulatedobserved) is also illustrated for the composite analysis of Figure 2. The ratio is spotty, with positive biases adjacent to negative biases in the western Atlantic and in the eastern Atlantic in mid-latitudes. We suspect that this structure is due to small of risets in the predicted positions of aerosol mass relative to observed values. Simulated optical depths are generally lower than observed, particularly in the subtropical Atlantic, where typical sate 1 .e values are on the order of 0.1 to 0.25 , and the modeled values are relatively uniform with values of 0.08 .

The ratio of simulated-observad optical depth decreases eastward across most of the central and eastern North Atlantic, reflecting the lower simulated optical loading over this part of the model domain. A possible source of bias is excessive precipitation scavenging in the simulation. The model treats the precipitation rates archived by the ECMWF forecast model every 6 hours as constant for each 6-hour period; a more realistic treatment of the sporadic nature of precipitation would remove less aerosol and hence yield higher simulated aerosol loading. Other factors contributing to the bias could be neglected aerosol types in the simulation (dust, sea salt, carbonaceous aerosol, volcanic aerosol) and neglect of aqueous chemistry in nonprecipitating clouds.

The ratio of simulated-observed optical depth is lowest in the subtropics, particularly just off the coast of West Africa, where it drops to 0.15 . Evidently desert dust, which is not represented in the simulations, is the dominant contributor to optical loading in the subtropical North Atlantic.

\section{Summary}

Although the results we are presenting must be regarded as preliminary, they demonstrate a general correspondence between simulations and observations in regions contaminated by anthropogenic sulfur aerosol. The biases evident in other regions can be explained in terms of either aerosols not considered in the simulation, or potentially correctable deficiencies in the sulfur model. Thus, we conclude that the approach we have taken is a viable method of evaluating aerosol models. However, we will not consider the evaluation complete until we have addressed the problems identified in this paper.

\section{References}

Luecken, D. J., C. M. Berkowitz, and R. C. Easter. 1991. "Use of a Three-Dimensional Cloud-Chemistry Model to Study the Transatlantic Transport of Soluble Sulfur Species."

J. Geophys. Res. 96:22477-22490.

Wagener, R., S. Nemesure, C. M. Benkovitz, S. E. Schwartz, C. M. Berkowitz, and S. J. Ghan. "Cloud-free Aerosol Optical Depth Determination over Oceans from Satellite Radiometry." Conference on Atmospheric Chemistry, AMS Annual Meeting, 23 - 28 January 1994, Nashville, Tennessee, American Meteorological Society.

\section{Presentations}

Benkovitz, C. M., C. M. Berkowitz, R. C. Easter, and S. E. Schwartz. "Fine Resolution Atmospheric Sulfate Model Driven By Operational Meteorological Data: Comparison with Observations, Conference on Atmospheric Chemistry." To be presented at the American Meteorological Society Annual Meeting, 23 - 28 January 1994, Nashville, Tennessee.

Berkowitz, C. M., and S. J. Ghan. "Evaluation of Sulfate Aerosol Optical Depths Over the North Atlantic and Comparison with Satellite Observations." American Meteorological Society Annual Meeting, 23-28 January 1994, Nashville, Tennessee. 

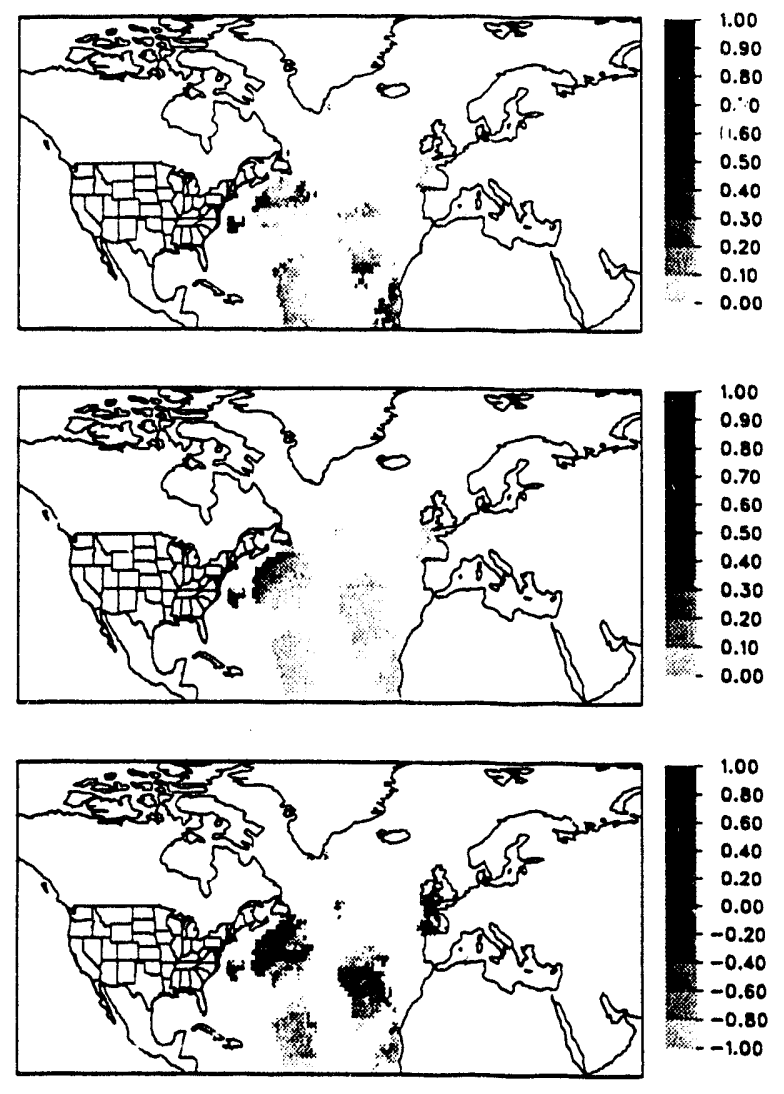

Figure 1. Observed (top) and Simulated (middle) Optical Depth and the $\log _{10}$ of the Rativ Simulated-Observed (bottom) for October 23, 1986
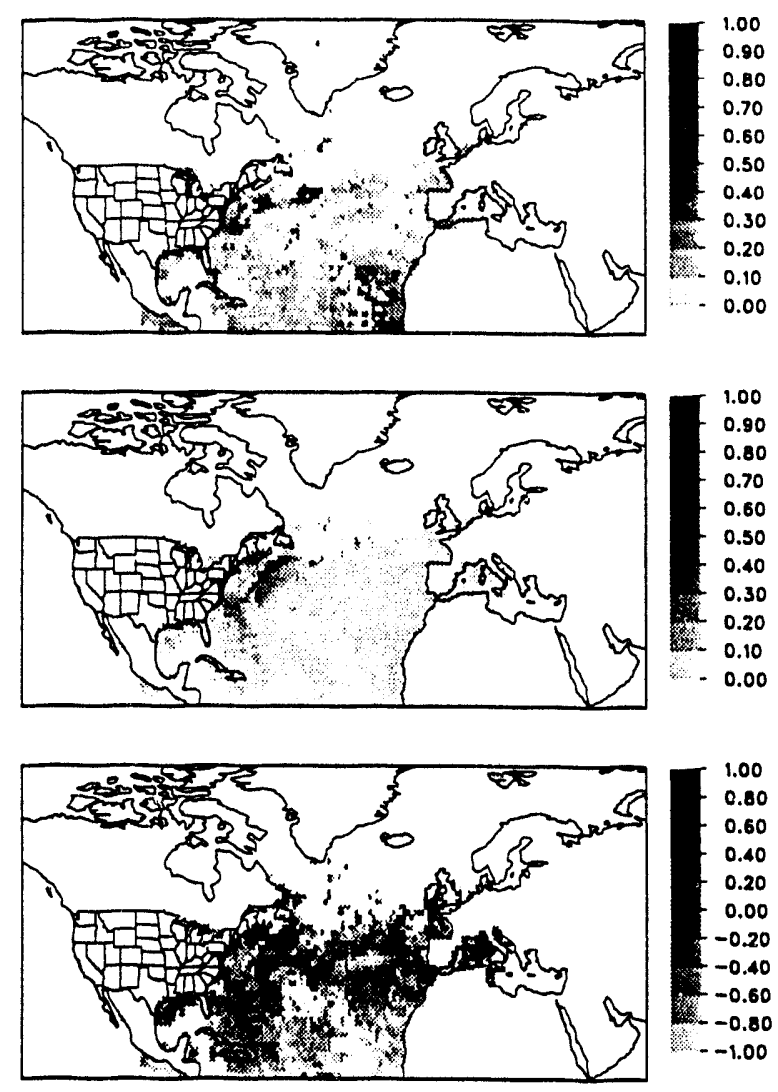

Figure 2. Composite Observed (top) and Simulated (middle) Optical Depth and the $\log _{10}$ of the Ratio Simulated-Observed (bottom) for the Period October 23-30, 1986 


\title{
Radiometric Characterization of Subvisual Cirrus Clouds
}

\author{
E. W. Kleckner (Geosciences)
}

Subvisual cirrus clouds may play an important role in radiation transfer in the atmosphere and in Global Climate Change; however, characterization of subvisual cirrus through ground-based measurements is lacking. We used Multiple Filter Rotating Shadowband Radiometers (MFRSRs) to explore the effects of these clouds on radiative measurements in the visible wavelength range. Three MFRSRs were programmed to take rapid sequences of total, diffuse, and direct solar irradiance at six wavelengths in the range 400 to $1000 \mathrm{~nm}$. Analysis of the data focused on small fluctuations in the direct and diffuse radiation. components. We emphasize that the outcome of this investigation was uncertain, as we have attempted to measure what has previously been considered "noise" in the direct solar beam signal. Our results show that we can identify subvisual cirrus clouds that pass within the vicinity of the sun in MFRSR data.

Subvisual, or optically thin, cirrus clouds in the vicinity of the sun cause a slight decrease in the direct beam component of irradiance, while potentially increasing the diffuse sky irradiance through enhanced forward scattering of photons. We identified such behavior in the data of three MFRSRs stationed near the Pacific Northwest Laboratory on May 24, 1993. The central unit was on top of Rattlesnake Mountain, and the other two units were located about 25 kilometers southeast and 100 kilometers northwest of the mountain, thereby establishing a suitable base line for discrimination of clouds near the tropopause ( $\sim 12$ kilometers altitude). The temporal behavior of atmospheric optical depths at these sites was studied for the appearance of thin-cloud signatures.

\section{Experiment Results}

The sky was clear at all three sites during the morning; this allowed us to determine atmospheric optical depths at the detectors' nominal wavelengths of $414,499,608,662,859$, and $934 \mathrm{~nm}$. These optical depths remained stable until approximately 14:00 Local Standard Time, when all three sites experienced an increase of about 0.025 optical depth at all wavelengths (seen near 0.9 UT Day Fraction in the accompanying figure that shows the deviation of optical depth from the morning value at one site). This is below the threshold optical depth of 0.05 for visual identification of cirrus clouds given by Lynch (1993), as well as the 0.03 threshold value suggested by Sassen et al. (1990) using visible lidar. Rather than being a sharp change in optical depth, the increase was gradual over about 20 minutes, followed by a gradual decrease toward lower optical depth, but never back to the low level seen previously. About 2 hours later, rapid increases in optical depth resulted from passage of visible cirrus clouds, as was verified by a field observer at the northwestern site. This behavior is exactly what is expected for the passage of an optically tenuous, subvisual cirrus cloud, followed by visible cirrus. These clouds were associated with a front approaching the area that had a surface passage time near 16:45 Local Standard Time the following day. Variations in the optical depth signals of the subvisual cloud indicate it was somewhat spatially heterogeneous over the approximately 125 -kilometers scale defined by the sites. Also, the measured shifts in observation times suggest the cloud may have been associated with a wave having a westward component of phase velocity.

\section{Conclusions}

We have identified subvisual cirrus clouds in data from the MFRSR instrument under generally clear sky conditions. These clouds were not easily seen in the raw data signals, but stood out well in the time series of calculated optical depth residuals. By comparison with field observation, cirrus clouds are not detectable by eye, as long as their optical depths remain under approximately 0.05 , in agreement with other studies.

From cursory analysis of the noise in the optical depth data, it is possible to detect real changes in optical depth that are limited only by atmospheric stability. At the 1088-meter altitude of Rattlesnake Mountain, coherent changes of 0.0025 optical depth were measurable; at lower altitudes fluctuations are observed to be somewhat greater. 
We conclude that an algorithm can be developed for MFRSR data that will allow measurement of subvisual cirrus cloud optical depths, thereby allowing us to assess their impact on the radiative balance of the atmosphere. Because volcanic aerosols have been observed to produce optical depth perturbations of the same order of magnitude as subvisual cirrus clouds, the effect of the presence of these clouds could have a more significant long-term impact on climate.

\section{References}

Lynch, D. K. 1993. "Subvisual Cirrus: What it is and where you find it." In Passive Infrared Remote Sensing of Clouds and the Atmosphere, ed. D. K. Lynch, Proceedings of SPIE, Volume 1934, pp. 264-274.

Sassen, K., M. K. Griffin, and G. C. Dodd. 1990. "Subvisual Cirrus Cloud Properties Derived From a FIRE IFO Case Study." In FIRE Science Results 1988, ods. D. S. McDougal and H. S. Wagner. Proceedings of NASA Conference, Publication 3083, pp. 85-88.

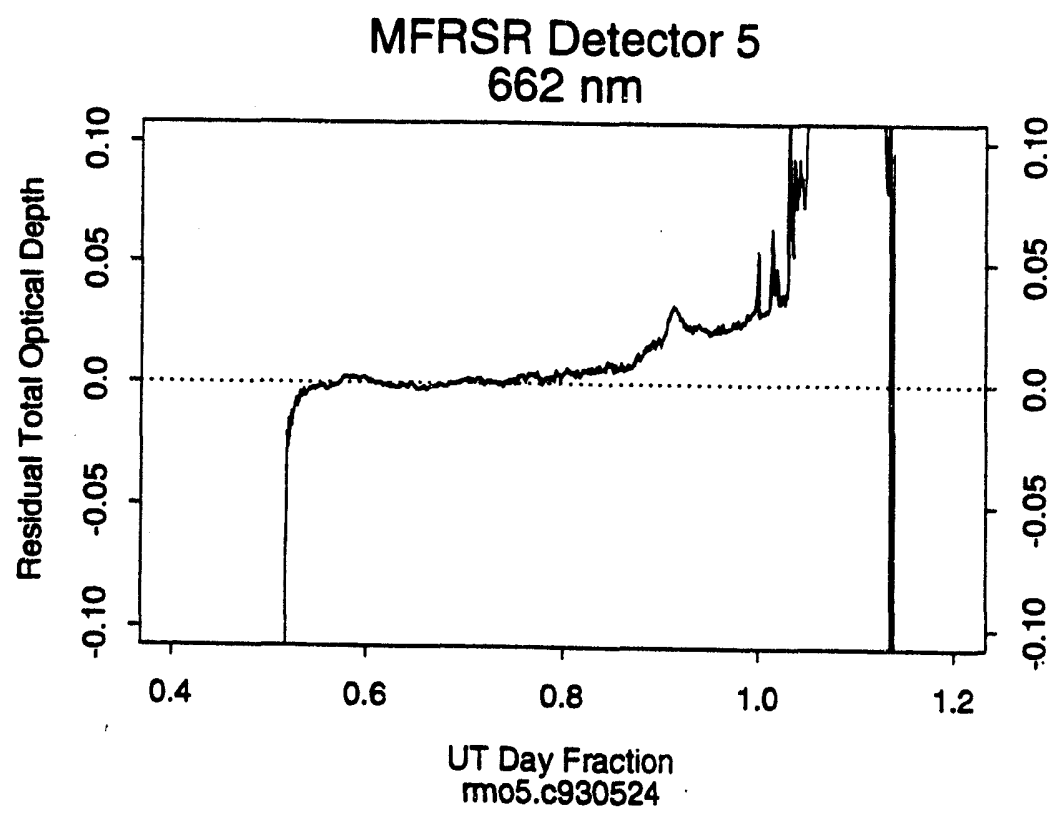

Figure 1. The Derivation of Optical Depth from the Morning Value 


\title{
Redesign of Biodegradative Enzymes: Theory Development and Coupled Theory-Experiment Approach
}

\author{
R. L. Ornstein (Theory, Modeling and Simulation)
}

Cost-effective remediation of synthetic substances in deep subsurface environments is, for the most part, limited to novel bioremediation alternatives, such as rational enzyme redesign. The unique advantage of rational redesign is its ability to foster discovery of new enzymatic forms that would otherwise have an extremely low probability of evolving biologically under either laboratorycontrolled or natural conditions (which is to be expected for an enzyme requiring many simultaneous and specific amino acid changes to efficiently catalyze a significantly different substrate). Some of the major prerequisites for rational enzyme redesign include a good starting experimental structure, an understanding of the enzyme mechanism, a cloned gene for the enzyme, and an understanding of the enzymes nonaverage structural and dynamic features. The latter are best studied by computer simulations, such as by molecular dynamics simulations.

Cytochromes P450 are a superfamily of monooxygenase enzymes that have been identified in prokaryotes, lower eukaryotes, insects, fish, higher eukaryotes, and plants, as well as many mammalian tissues and cell types. The ubiquitous distribution of cytochromes P450 is equalled by the variety of molecules which are their substrates and the variety of reactions they are capable of catalyzing. Cytochromes P450 have been shown to be capable of oxidative and/or reductive dehalogenation depending on the substrate and reaction conditions.

Until 1993, the only available crystal structure of a P450 is that of P450cam from Pseudomonas putida; this enzyme initiates the conversion of camphor to 5-exo-hydroxycamphor. The functional enzyme system consists of a cytochrome P450 heme protein and two proteins that convey electrons from nicotinamide adenine dinucleotide phosphate or nicotinamide adenine dinucleotide to the cytochrome. The active center of the P450 is the iron protoporphyrin moiety and an adjoining apoprotein. The mechanistic pathways and intermediates for P450 catalysis are reasonably well understood having been explored experimentally and by theoretical quantum-chemical studies. P450cam from Pseudomonas putida has been cloned and expressed in Escherichia coli. Recent sitedirected mutagenesis studies have been successfully applied to P450cam.

Theory Development, Validation, and Optimization for Protein Simulations

Although enzyme structures determined by $x$-ray crystallography or solution nuclear magnetic resonance methods are invaluable for rational design, they generally suffer from the following limitations:

- The structural image obtained is an average in time and space and therefore some atomic positions may be "virtual reality" due to mathematical averaging.

- Conformational details are usually unrecoverable for structures less than 5\% to $10 \%$ in population. Understanding these lower populated conformations is important since bioactivity is not necessarily correlated with percent (of structural) population.

- Molecular surface properties are often significantly deformed due to crystal packing in the solid-state, or due to solution differences from those of in vivo conditions, such as when the $\mathrm{pH}$ is significantly lowered or raised, or when various agents are added to reduce precipitation at high concentrations of protein. Computational simulation methods, starting from a high-resolution experimental structure, can potentially help resolve these experimental structural limitations, as well as lead to a better understanding of the dynamic mobilities and fluctuations inherent in enzyme structure and function.

With today's supercomputers and conventional molecular simulation methods, state-of-the-art enzyme 
simulations can be performed for about a nanosecond of simulated time. Simulations of this length represent the "tip-of-the-iceberg" with respect to uncovering functionally interesting motions and conformational transitions. Therefore, reasonably accurate and long time duration simulations are important milestones of this effort. We have made considerable progress in this area; see for instance publications and references: Arnold and Ornstein 1992, 1993; Bass et al. 1992a; Braatz et al. 1992; Paulsen and Ornstein, 1991, 1992, 1993; Ornstein 1990.

\section{Coupled Theory-Experimental Approach}

It is widely accepted that coupled theory-experimental studies will be required if enzyme structure-function is to be adequately understood in order to accomplish rational redesign goals. Our studies in this area follow a progression from 1) determining the level of agreement between computed and experimental observables using currently available methods; 2) using newly acquired structure-function-dynamics relations to make experimentally testable hypotheses (i.e., proof-of-principle); and 3) initiating environmentally relevant target rational redesign. Examples of progress made in these areas are noted below: 1) Bass et al. 1992b; Filipovic et al. 1992; Paulsen et al. 1991; Paulsen and Ornstein 1991, 1992, 1993; 2) Bass et al. 1992c; Paulsen et al. 1993. Work is under way in area 3).

\section{Future Prospects}

With the growing number of three-dimensional structures of enzymes determined by $\mathrm{x}$-ray crystallography and the prospects of high-field nuclear magnetic resonance enzyme structure determination, rational design can be expected to play an increasing role in enzyme engineering for enhanced subsurface remediation, as well as a wide-range of other biotechnological uses, see Ornstein 1994.

\section{References}

Arnold, G. E., and R. L. Ornstein. 1992. "A Molecular Dynamics Simulation of Bacteriophage T4 Lysozyme." Protein Engineering 5:703-714.

Bass, M. B., D. F. Hopkins, W. A. Jaquysh, and R. L. Ornstein. 1992. "A Method for Determining the Positions of Polar Hydrogens Added to a Protein Structure that Maximizes Protein Hydrogen Bonding " PROTEINS 12:266-277.
Bass, M. B., M. D. Paulsen, and R. L. Ornstein. 1992. "Molecular Dynamics Simulations of Norcamphor-Cytochrome P-450cam and Mutations of P-450cam Designed to Alter the Product Specificity." In Cytochrome P-450: Biochemistry and Biophysics, eds. A. I. Archakov and G. I. Bachmanova, pp. 680-685, INCO-TNC, Moscow, Russia.

Bass, M. B., M. D. Paulsen, and R. L. Ornstein. 1992. "Substrate Mobility in a Deeply Buried Active Site: Analysis of Norcamphor Bound to Cytochrome P-450cam as Determined by a 201 psec Molecular Dynamics Simulation." PROTEINS 13:26-37.

Brantz, J. A., M. D. Paulsen, and R. L. Ornstein. 1992. "3 nsec Molecular Dynamics Simulation of the Protein Ubiquitin and Comparison with X-ray Crystal and Solution NMR Structure." J. Biomolec. Struct. \& Dyn. 9:935-949.

Filipovic, D., M. D. Paulsen, P. J. Loida, S. G. Sligar, and R. L. Ornstein. 1992. "Ethylbenzene Hydroxylation by Cytochrome P450cam." Biochem. Biophys. Res. Commun. 189:488-495.

Ornstein, R. L. 1990. "Using Molecular Dynamics Simulations on Crambin to Evaluate the Suitability of Different Continuum Dielectric Models for Protein Simulations." J. Biomolec. Struct. \& Dyn. 7:1019-1041.

Paulsen, M. D., M. D. Bass, and R. L. Ornstein. 1991. "Analysis of Active Site Motions from a $175 \mathrm{psec}$ Molecular Dynamics Simulation of Camphor-Bound Cytochrome P450cam." J. Biomolec. Struct. \& Dyn. 9:187-203.

Paulsen, M. D., and R. L. Ornstein. 1991. "A $175 \mathrm{psec}$ Molecular Dynamics Simulation of Camphor-Bound Cytochrome P450cam." PROTEINS 11:184-204.

Paulsen, M. D., and R. L. Ornstein. 1992. "Predicting Product Specificity and Coupling for Catalysis by Cytochrome P450cam." J. Computer-Aided Molec. Design 6:449-460.

\section{Publications}

Arnold, G. E., and R. L. Ornstein. "An Evaluation of Implicit and Explicit Solvent Model Systems for the Molecular Dynamics Simulation of Bacteriophage T4 Lysozyme." Proteins, in press.

Bass, M. B., and R. L. Ornstein. 1993. "Substrate Specificity of Cytochrome P-450cam for L- and D-Norcamphor as Studied by Molecular Dynamics Simulations." J. Comp. Chem. 14:541-548.

Manchester, J. I., M. Shibata, R. F. Setlik, R. L. Ornstein, and R. Rein. "Applicability of PM3 to Transphosphorylation Reaction Path: Toward Designing a Minimal Ribozyme." Origins of Life and Evolution of the Biosphere, in press. 
Ornstein, R. L. "On Using Rational Enzyme Redesign to Improve Enzyme-Mediated Microbial Dehalogenation of Recalcitrant Substances in Deep-Subsurface Environments." In Structural Biology: State of the Art 1993, Proceedings of the Eighth Conversations, eds. R. H. Sarma and M. H. Sarma, Adenine Press, Albany, New York, in press.

Paulsen, M. D., D. Filipovic, S. G. Sligar, and R. L. Ornstein. 1993. "Controlling the Regiospecificity and Coupling of Cytochrome P450cam: T185F Mutant Increases Coupling and Abolishes 3-Hydroxy-norcamphor Product." Protein Science 2:357-365.

Paulsen, M. D., and R. L. Ornstein. 1993. "Substrate Mobility in Thiocamphor-Bound Cytochrome P450cam: An Explanation of the Conflict Between the Observed Product Profile and the X-ray Structure." Protein Engineering 6:359-365.
Setlik, R. F., R. Garduno, J. I. Manchester, M. Shibata, R. L. Ornstein, and R. Rein. 1993. "Modeling Study on the Cleavage Step of the Self-Splicing Reaction in Group 1 Introns." J. Biomolec. Struct. and Dyn. 10:945-972.

Sokalski, W. A., D. A. Keller, R. L. Ornstein, and R. Rein. 1993b. "Effects of the Multipole Correction on Charge Distribution: I. Peptides." J. Comput. Chem. 14:970-976.

Sokalski, W. A., M. Shibata, R. L. Ornstein, and R. Rein. 1993a. "Point Charge Representation of Multicenter Multipole Moments in Calculation of Electrostatic Properties." Theoret. Chim. Acta 85:209-216.

Other Accomplishments

M. D. Paulsen (Ph.D. in Physical Chemistry, University of Wisconsin, Madison) was hired in FY 1993 and G. E. Arnold (Ph.D. in Biochemistry, Washington State University, Pullman) is to be hired in FY 1994. 


\title{
Remotely Piloted Vehicle for Global Climate Change Measurements
}

\author{
C. D. Whiteman (Atmospheric Sciences)
}

The objective of this LDRD project was to develop a remotely piloted aircraft to collect meteorological and environmental data in the atmospheric boundary layer. The aircraft was designed to be piloted from a computer screen using an onboard forward-looking video camera, with video and aircraft attitude data relayed to the ground through video and audio data links. An onboard autopilot and a global positioning system navigation system were included in the design so that the aircraft could fly a preprogrammed course under autopilot control. Meteorological data are recorded onboard through a central processing unit that controls a programmable data acquisition system. The aircraft design was completed and all subsystems were assembled and tested in FY 1993, but field testing of the full system was not completed.

A small remotely piloted vehicle (RPV) was developed to collect in situ atmospheric measurements in support of atmospheric research in the Global Climate Change program. The low-cost remotely piloted vehicle was designed to carry a meteorological sensor payload while using the global positioning system to fly a preprogrammed course under autopilot control. Over the last few years, new technology has become available from U.S. Department of Defense (DOD) programs to develop drones or unmanned aerospace vehicles that can be used in atmospheric research. Previous remote-control meteorological drones have been used to collect $a$ ' $a$ in the atmospheric boundary layer within sight of the remote-control operator (Martin 1980), but recently subsystems have become available (including the global positioning system) that can be combined in a small autonomous aircraft that can be flown under autopilot control on a preprogrammed course. Several existing projects are focusing development efforts on large unmanned aircraft (Langford and Emanue' ‘ 33; Taubes 1993).

The Perseus A drone, for example, is under development to collect ozone data in the stratosphere on multiday flights (Taubes 1993). A successor craft to Perseus, called Theseus, may be used to gather data for hurricane forecasts (Wingo 1992). The Boeing Condor has been designed to attain altitudes of 20 kilometers on multiday flights to distances of 20,000 kilometers. A smaller aircraft, the aerosonde, is under development by a private company to make atmospheric soundings at any place on the globe to elevations of 14 kilometers (Holland et al. 1992). Other DOD contractors are developing unmanned aerospace vehicles and/or robotics platforms that can be flown through radioactive clouds or used to assess environmental contamination or to design cleanup activities at contaminated sites.

Our project is distinguished from these other ongoing development efforts by the design characteristics, which are focused on the needs of the Global Climate Change program for meteorological boundary layer and radiometric data over distances of 10 kilometers and altitudes less than 1 kilometer.

Our project has assembled a low-cost remotely piloted vehicle that can be flown from a ground console. System subcomponents include

- A personal computer-based groun : console that provides radio, video, and data links to the remotely piloted vehicle. The pilot would use this console to fly the remotely piloted vehicle and to collect, process, and store attitude and position data.

- A miniature onboard video camera system that would relay aircraft altitude, airspeed, heading, and operational status to the pilot during flight. With proper Federal Aviation Administration approval, the remotely piloted vehicle could be flown in clouds or in other areas where the remotely piloted vehicle is out of sight of the aerodrome.

- An autopilot system that allows the aircraft to be flown on a straight and level course without active flight direction from the pilot. 
- A global positioning system navigation system that allows the remotely piloted vehicie track, position, ground speed, and other data to be relayed to the console.

- An onboard microprocessor that stores the control surface instructions to allow the airplane to make turns at selected waypoints without assistance from the pilot.

- An onboard data acquisition system that collects and stores meteorological and aircraft position data.

After an initial FY 1992 test flight in which the aircraft crashed, we $r$ 'esigned the airframe to make it more airworthy. We investigated the use of nets or parachutes to recover the aircraft at the end of each flight instead of landing it under operator control. The detailed mechanical and electronics design of the remotely piloted vehicle was finished in FY 1993. Schematics of the airborne and ground-based portions of the system are shown in Figures 1 and 2. A 4-cycle engine was chosen to power the vehicle, as this engine reduces noise, vibration, and iuel consumption. A video data link and camera were purchased and tested in the laboratory to provide the pilot with a cockpit view during takeoff and landing. The associated audio channel is used to send digital data to the pilot to report aircraft status and flight parameters, including airspeed and heading. An airborne meteorological sensor package was developed and tested. This package, with signal conditioning, includes a pitot tube for airspeed measurements, a thermistor for temperature measurements, a capacitive humidity sensor, and a pressure sensor. A new onboard microprocessor was procured and tested to allow three different flight modes. First, the pilot can override the microprocessor to assume manual flight control during takeoffs and landings. Second, the aircraft can be flown in a flight record mode, is which the aircraft control surface settings are recorded while the aircraft is under manual control. In this way, the position of the control surfaces during flight operations such as turns can be recorded for entry into computer programs that control the aircraft when it is under microprocessor control. Third, the aircraft can be flown to preprogrammed waypoints solely under computer control. All subsystems (airframe, aircraft power plant, remote control and servos, global positioning system, microprocessor, meteorological package, video camera, video, and links) were individually tested in FY 1993. The approach to testing has been to add subsystems to the airframe incrementally as we gained flight and operational experience. We have now completed initial airframe test flights and are beginning flight tests with the other subsystems. Further testing and development of the remotely piloted vehicle will be necessary to field an operational system.

\section{References}

Holland, G. J., T. McGeer, and H. Youngren. 1992. "Autonomous Aerosondes for Economical Atmospheric Soundings Anywhere on the Globe." Bull. Amer. Meteor. Soc. 73:1987-1998.

Langford, J. S., and K. A. Emanuel. 1993. "An Unmanned Aircraft for Dropwindsonde Deployment and Hurricane Reconnaissance." Bull. Amer. Meteor. Soc. 74:367-375.

Lovece, J. 1993. "Weston, IAI Target Civilian Cleanup Market with Unmanned Defense Drones." Environ. Week 11 March 1993.

Martin, D. 1980. "Remotely Piloted Aircraft for Atmospheric Soundings." The Boulder Low-Level Intercomparison Experiment. Rep. No. 2, eds. J. G. Kaimal, H. W. Baynton, and J. B. Gaynor, NOAA/NCAR Boulder Atmospheric Observatory. 18-25.

Taubes, G. 1993. "NASA Launches a 5-Year Plan to Clone Drones." Science, 260, 16 April 1993, 286.

Wingo, W. S. 1992. "PERSEUS Scouts the Ozone." Design News, 7 September 1992, 70-76. 


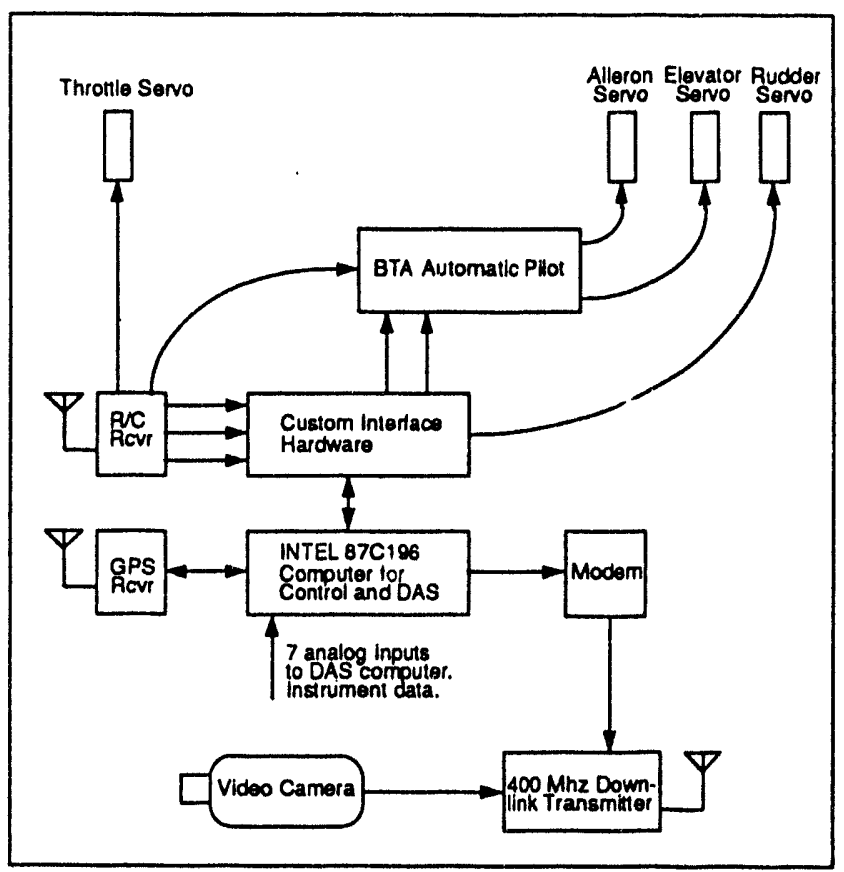

Figure 1. Remotely Piloted Vehicle Airborne Electronics

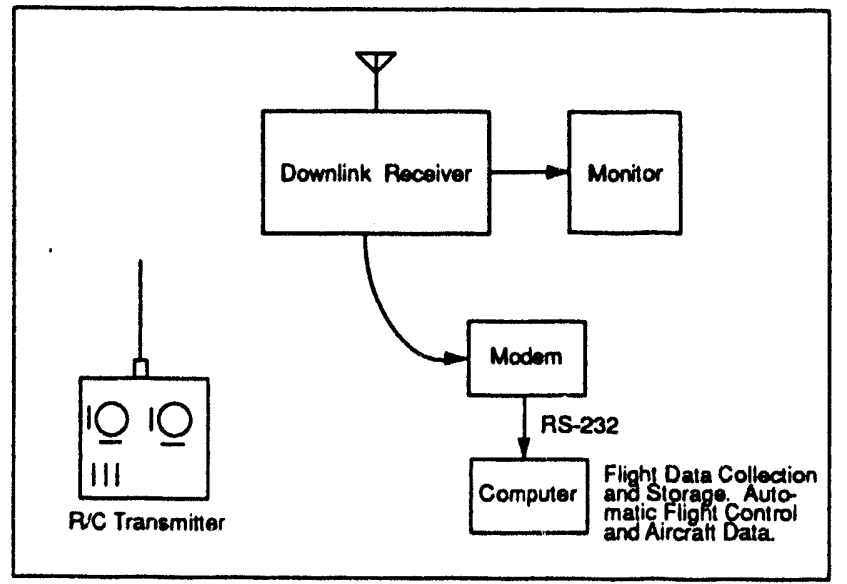

Figure 2. Remotely Piloted Vehicle Ground Control Electronics 


\title{
Site-Specific DNA Damage by Radiation and Chemicals
}

\author{
D. L. Springer and B. D. Thrall (Biology and Chemistry)
}

The objective of this project was to develop and test a model system which, at a mechanistic level, could be used to study DNA damage by radiation and chemicals. Specifically, we proposed to develop and implement methods which would allow for structural and functional studies of damage in DNA containing single, site-specific lesions.

Conformational effects due to either the presence of DNA lesions or to local DNA sequence context may significantly alter the susceptibility of the DNA to damage by radiation or chemicals. Such conformational effects could be reflected in either sequencerelated alterations in the type or yield of damage or possibly interactions between different types of DNA damaging agents. These hypotheses are difficult to study in the complex environment of genomic DNA and require model DNA systems with single, sitespecific lesions which can be analyzed at single base resolution. Thus, we have initiated studies using oligonucleotides which can be modified in a sitespecific and stereospecific manner by DNA-damaging agents. These model systems allow for a homogeneous and well-defined population of damaged DNA for studies of the biological effects of such damage and ultimately the interrelationship between structure and function in mutagenesis and carcinogenesis.

During FY 1993, we have developed procedures for constr' cting oligonucleotides with a single, sitespecific adduct with the model carcinogen benzo[a]pyrene diol epoxide (BPDE). Racemic mixtures of benzo[a]pyrene diol epoxide bind to DNA with high preference toward guanines and form a number of guanine adducts which differ only by stereochemistry. Thus, the isolation of individual oligonucleotides with a homogeneous population of adducts requires the ability to purify modified DNA based on stereochemical differences. To begin these studies, we have synthesized oligonucleotides containing a single guanine which can be modified by benzo[a]pyrene diol epoxide. In addition, reversed-phase high pressure liquid chromatography methods were implemented to purify the nonadducted DNA from the DNA containing adducts. Figure 1 shows an example of a highpressure liquid chromatography separation of a 17 -mer modified with the (-)-anti-benzo a]pyrene diol epoxide enantiomer. This illustrates the ability to separate not only nonadducted and adducted oligonucleotides, but to separate stereochemically defined adducts (cis and trans). The stereochemical configuration of these adducts was further confirmed by ultraviolet spectrophotometry, and the site of adducts was confirmed to be at the single guanine residue using enzymatic procedures. These procedures have provided the essential tools needed for studying the interrelationship between structure and biological consequences of DNA adducts at single base resolution using a homogeneous population of damaged DNA.

In addition to DNA damage by chemical agents, preliminary experiments to analyze DNA damage by ionizing radiation using oligonucleotide model systems were conducted. The local DNA sequence context is known to influence the susceptibility of the DNA to damage by chemicals, including benzo[a]pyrene diol epoxide; presumably due to local sequence-directed structural variations. However, the role of DNA sequence in damage produced by ionizing radiation is not understood. To address this possibility, a preliminary experiment was conducted in collaboration with Dr. A. F. Fuciarelli to determine the effect of DNA sequence on thymine glycol formation after irradiation with ${ }^{60} \mathrm{Co}$. Oligonucleotides (20-mers) containing a single thymine at different positions were irradiated in air and the relative yield of thymine glycol was analyzed by gas-chromatography/mass spectrometry with selected ion monitoring. In this preliminary experiment, we found that the yield of thymine glycol varied by nearly fivefold in the various oligonucleotides irradiated under identical conditions, although a particular sequence-related context which correlates with this response was not obvious. Although further studies are needed to verify these results, it appears possible that damage to DNA by radiation may also vary in different DNA sequence contexts.

It has been suggested that DNA damage may be influenced by the presence of existing DNA damage which 
alters the normal structure of the DNA, or by preexposure of the cells to DNA damaging agents. We have conducted experiments using a plasmid DNA system, modified with racemic benzo[a]pyrene diol epoxide to determine if benzo[a]pyrene diol epoxide adducts interact with ionizing radiation to generate DNA strand breaks. Plasmids containing approximately 1.0 adduct $/ \mathrm{kb}$ were irradiated at 0 to $10 \mathrm{~Gy}{ }^{60} \mathrm{Co}$, and the number of strand breaks were analyzed by agarose gel electrophoresis. Under these conditions, we found similar numbers of strand breaks in adducted and nonadducted DNA, with no apparent evidence for interactions. Additionally, we have conducted studies in human cells to determine if preexposure to ionizing radiation influenced the efficiency of adduct formation by benzo[a]pyrene diol epoxide. Human K562 cells were exposed to 0 to $2 \mathrm{~Gy}{ }^{60} \mathrm{Co}$ followed 30 minutes later by treatment with $0.5 \mu \mathrm{M}{ }^{3} \mathrm{H}-\mathrm{BPDE}$. Similar levels of benzo[a]pyrene diol epoxide adducts were found in both controls and pre-irradiated cells. Thus, under these experimental conditions, we did not find evidence for interactions between benzo[a]pyrene diol epoxide and ionizing radiation. However, these experiments do not exclude the possibility that radiation and benzo[a]pyrene diol epoxide may interact in other manners which were not detectable by these experiments.

Our efforts toward developing oligonucleotites containing site-specific and stereo-specific DNA damage have provided a tool which can be used to further investigate the role between structure and biological consequences of DNA damage. Using these model oligonucleotides, we have initiated experiments to determine how DNA polymerases, enzymes which replicate DNA, process damaged DNA. Errors in replication of DNA containing DNA adducts could result in mutagenic changes which are important to tumor initiation. For these studies, a 17-mer containing a single guanine was modified with benzo[a]pyrene diol epoxide, and purified by highpressure liquid chromatography (see Figure 1). A primer (12-mer) was annealed to the modified 17-mer, and the ability of DNA polymerases to replicate (primer extension) was analyzed by gel electrophoresis. Figure 2 illustrates a densitometric scan of the products obtained after gel electrophoresis.

Using cloned T7 DNA polymerase (Sequenase, U.S. Biochemical), we find that the polymerase can bypass a large fraction of the adducted guanines and produce full-length DNA. Thus, "translesional" synthesis may be an important mechanism by which certain benzo[a]pyrene diol epoxide adducts are mutagenic, and we suggest that the structure of the individual adducts may be important to these processes.

Experiments to further characterize the biological consequence of these lesions are under way. Included among these efforts are experiments using modified oligonucleotides and human cell extracts to study mechanisms by which these lesions are repaired. In addition, this work may identify novel proteins which may be involved in repair of bulky chemical adducts. Studies of adduct structure, using high-field solution nuclear magnetic resonance will also be initiated in collaboration with Dr. M. A. Kennedy (MSRC). These studies will increase our understanding of the role that structure plays in the biological processing and consequences of covalent DNA damage.

\section{Publication}

Thrall, B. D., and D. L. Springer. "Repair of DNA Adducts from ( \pm -Benzo[a]pyrene Diol Epoxide by Human Nuclear Extracts." Mutation Res, in preparation.

\section{Presentation}

Thrall, B. D., and D. L. Springer. 1993. "Translesional Synthesis by Model Polymerases on an Oligonucleotide Modified with Benzo[a]pyrene Diol Epoxide." Presented at the 9th Annual Meeting for the Pacific Northwest Association of Toxicology.

\section{Other Accomplishments}

In January 1993, Dr. Brian Thrall was hired and has provided approximately $21 \%$ effort on this project. 


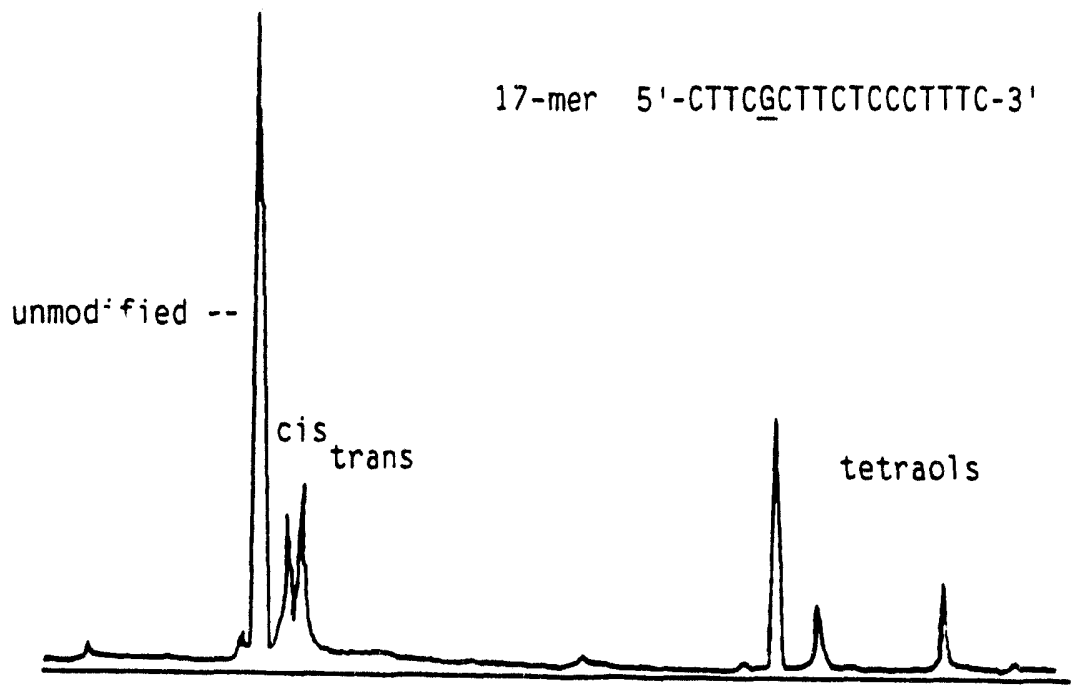

Figure 1. High-Pressure Liquid Chromatography Separation of a 17-mer Modified with (-)-anti-BPDE. A 17-mer containing a single guanine residue was synthesized and reacted with (-)-anti-BPDE. Reversed-HPLC separation methods were implemented to separate not only adducted DNA from nonadducted, but also to separate sterospecific (cis and trans) adducted oligonucleotides.

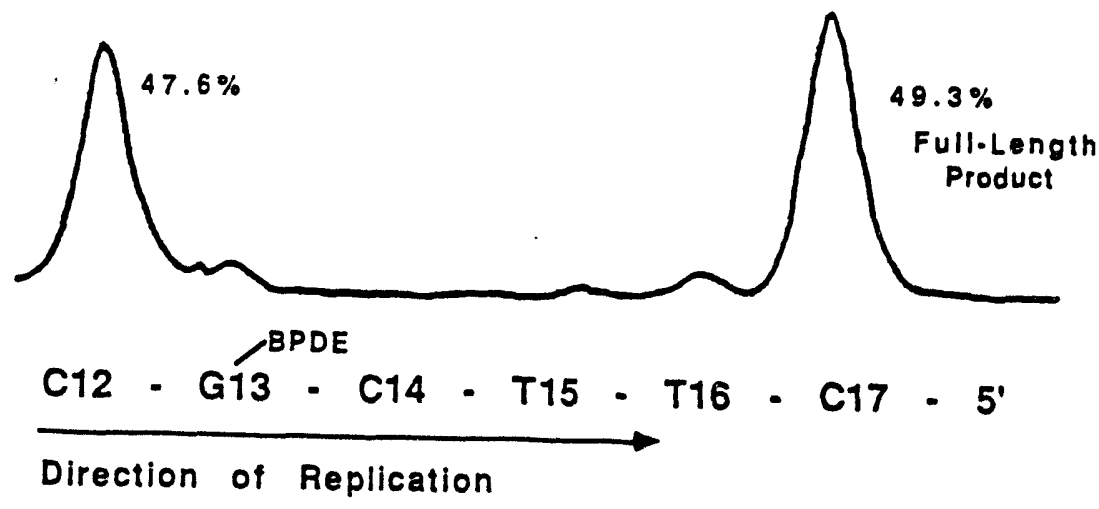

Figure 2. Translesional Synthesis on a 17-mer Modified by (-)-anti-BPDE. The 17-mer described in Figure 1 was synthesized and modified with (-)-anti-BPDE, and adducted oligonucleotides containing a modified guanine at position 13 were purified by HPLC. A 12-base primer extension was annealed, and the template was replicated using closed T7 DNA polymerase. The products of the primer extension were separated by electrophoresis and quantitated by scanning densitometry. A significant (49\%) portion of the modified templates were replicated to full-length DNA, indicating the polymerase can "bypass" some BPDE adducts during replication. 


\title{
Solid Waste Macro Material Flow Modeling
}

\author{
G. M. Holter (Waste Systems)
}

This project is providing new advances in predictively modeling the long-term impacts of solid waste management practices, thus establishing a basis for the analysis of alternative scenarios. During FY 1993, an architecture for the macro materials flow model was developed, and a simple prototype program demonstrating the planned operation of the full model was initiated; efforts at developing the necessary supporting data, which began in FY 1992, also continued. Full implementation and application of the model, when achieved, will provide a systematic basis for evaluation of solid waste production, management, and disposal.

Solid wastes are inevitable byproducts of human activities. Every segment of our society is involved in the generation of these wastes and, consequently, the activities generating the wastes and the compositions of the wastes are very diverse. These diverse waste products represent a complex threat to the environment but, at the same time, present opportunities to recover usable materials and/or energy from them.

The primary goal of this project was to identify and propose approaches to address current and future impacts at the regional, national, and international level that can result from solid waste management practices. Although solid waste management decisions are made predominantly at the local, county, and state levels, the cumulative result of such local decisions can have much broader impacts. Examples of relevant categories of impacts are resource depletion, environmental degradation, and reduced national competitiveness (through economic inefficiencies). There is currently a lack of a systematic approach to solid waste management at all levels. This project is intended to address this by providing the necessary basis and the analytical tools to conduct systematic evaluations of the performance of the solid waste management system under various scenarios.

During FY 1992, the basic direction and approach for macro material flow modeling were identified to provide a basis for understanding and analyzing the solid waste management system and the associated materials production and use cycle. This direction and approach were developed into a modeling concept and documented in a draft report; this report was completed and published during FY 1993. In addition, during FY 1992, a preliminary evaluation was conducted of available data on solid wastes to support the modeling activities.

FY 1993 activities were largely a continuation of those initiated in FY 1992. Development of a simple prototype macro material flow model was initiateci, based on the concept developed during FY 1992. When completed (expected in FY 1994), this prototype will simulate major portions of the macro material flow model functionality, and it is being developed to provide a basis for presenting and refining the model concept, confirming the operability of the model and the usefulness of the results, and facilitating liaison activities with prospective clients.

The macro material flow modeling concept and approach being pursued will provide a total-systemsoriented basis for evaluating potential impacts from various solid waste management system configurations and operating scenarios. This concept and approach include the entire life cycle of the major materials that are eventually identified as wastes, with consideration of the patterns of production and consumption that give rise to the wastes. This capability to model material flows can later be coupled with other types of information to calculate the full range of impacts from solid waste management activities.

Significant data to support the modeling effort were also collected. Information continues to be collected about quantities and compositions of solid waste materials. The information is then correlated with the origins of these wastes, in terms of sector of the economy and the function producing the wastes, to develop functional relationships allowing calculation of future waste quantities and compositions.

A multidimensional analysis of the solid waste management problem in the U.S. was also conceived and initiated, including an assessment of solid waste technology needs. Contacts within the solid waste 
area were pursued, to ensure that initiative efforts were being appropriately scoped to deal with actual problems and issues of concern.

Several technical papers addressing the technical approach and the modeling concept, as well as other related topics, were presented at national and international conferences on solid waste management and secondary materials.

In addition to the predictive modeling work, this project is also supporting the development and deployment of new concepts for approaching solid waste management problems. The major example of this is the continuing co-sponsorship of the concept for the Advanced Recycling and Research Complexes (ARRC), which would close the recycling loop by co-locating materials recyclers and customers for recycled materials, together with necessary ancillary capabilities, in an industrial park setting, to promote increased use of secondary materials within our society. The Advanced Recycling and Research Complexes concept has received considerable attention, both regionally and nationally, as an approach to enhancing opportunities for materials recycling and circumventing some of the barriers to recycling that exist in current practices.

\section{Publication}

Holter, G. M., and D. C. Stapp. 1993. Solid Waste Initiative Macro Material Flow Modeling Conceptual Description and Requirements, PNL-8470, Pacific Northwest Laboratory, Richland, Washington.

\section{Presentations}

Holter, G. M. 1993. "Pacific Northwest Laboratory's Solid Waste Initiative." Presented at Addressing Solid Waste Problems in the Pacific Northwest: Working Together to Reach Solutions, Richland, Washington. PNL-SA-22991.
Holter, G. M., K. A. Pennock, and S. R. Shaver. 1993. "Macro Material Flow Modeling for Analyzing Solid Waste Management Options." Presented at the 86th Annual Meeting of the Air \& Waste Management Association, Denver, Colorado. PNL-SA-21415.

Holter, G. M., D. C. Stapp, and J. E. Beck. 1992. "A Comprehensive Definition and Approach to Waste Minimization." Prasented at the 8th International Conference on Solid Wasto Management and Secondary Materials, Philadelphia, Pennsylvania. PNL-SA-20888.

Kuusinen, T. L., J. E. Beck, and G. M. Holter. 1992. "Regional Secondary Resource Utilization Parks: The Industrial Parks of the Future." Presented at the 8th International Conference on Solid Waste Mamagement and Secondary Materials, Philadelphia, Pennsylvania. PNL-SA-20892.

Stapp, D. C. September 1993. "The Concept of a Regional Partnership Focused on Solid Waste." Presented at Addressing Solid Waste Problems in the Pacific Northwest: Working Together to Reach Solutions, Richland, Washington. PNL-SA-23061.

Stapp, D. C., and G. M. Holter. 1992. "Solid Waste Research and Development: A Perspective from Pacific Northwest Laboratory." Presented at the 8th International Conference on Solid Waste Management and Secondary Materials, Philadelphia, Pennsylvania. PNL-SA-20905.

Other Accomplishments

This project directly supported a NORCUS student during the summer of 1993. A senior engineer was also hired to work part-time on this project because of his expertise and extensive business contacts within the solid waste area. 


\title{
Solution Crystal Growth
}

\author{
J. P. LaFemina (Materials and Interfaces)
}

The objective of this project was to perform fundamental research to develop the theoretical models necessary to describe the solution growth and dissolution processes for oxide and carbonate minerals. The materials and processes are important in the diverse areas of the transport of groundwater contaminants and the creation of advanced biomimetic materials.

The successful application of solution growth models hinges on their ability to reproduce interfacial (and ultimately interphasial) properties. Consequently, the first phase of this project concentrated on the development and testing of the theoretical models needed for the description of oxide and carbonate mineral surface properties.

In this work, new theoretical models were developed to investigate the surface properties of the $\mathrm{SnO}_{2}$ (110) and (111) surfaces and interfaces. In addition to developing models for predicting the surface atomic and electronic structure of these surfaces for comparison with experimental efforts in this area nngoing at PNL, these studies were able to elucidate the driving forces that control surface structure and bonding in these systems. These findings, coupled with previous work in the area of oxide surface structure, allowed for the development of a set of general principles which govern the relaxations and reconstructions of mineral oxide surfaces. These principles, in turn, allow for the qualitative prediction of the relative stability, and potential relaxations or reconstructions, of oxide surfaces.
One of the most important results to emerge from this study is that the set of principles which govern "ionic" oxide surface structure are remarkably similar to those derived for "covalent" semiconductor surfaces, indicating that the principles have a common basis that is more fundamental than the traditional and simple concepts of ionic versus covalent bonding. This may have profound implications for the chemistry which takes place on these surfaces. We plan to continue work in this particular area.

\section{Publications}

Godin, T. J., and J. P. LAFemina. "Atomic Structure of the Cassiterite (111) Surface." Surf. Sci., in press.

Godin, T. J., and J. P. LaFemina. 1993. "Surface Atomic and Electronic Structure of Cassiterite $\mathrm{SnO}_{2}$ (110)." Phys. Rev. B 47:6518.

LaFemina, J. P. "Theory of Insulator Surfaces." In Handbook of Surface Science: Physical Structures, ed. W. N. Unert, Elsevier, Amsterdam, in press.

\section{Presentations}

Godin, T. J., and J. P. LaFemina. 1993. "Atomic Structure of the $\mathrm{SnO}_{2}$ (111) Surface." Presented at the March 1993 Meeting of the American Physical Society, Seattle, Washington.

LaFemina, J. P., and T. J. Godin. 1992. "Application of Tight-Binding, Total-Energy Techniques to Mineral Oxide Surface Atomic Structures." Presented at the Fall 1992 Meeting of the American Geophysical Union, San Francisco, California. 


\title{
Spatial Data Visualization for Global Change
}

\author{
M. E. White (Computer Sciences)
}

Currently geographic information systems rely on static graphics which change only when a new entity or attribute is selected from the data base and displayed. For the geographic information systems to function as a spatial data management engine for modeling purposes requires a dynamic graphics approach. This project will look at linking a geographic information system to a dynamic visualization system and to an actual modeling effort.

4-DIVAS is a project directed at integrating the SYSTEM 9 Geographic Information System with the Application Visualization System geometric visualization system. While SYSTEM 9 has an objectoriented spatial data model, its attribute data is held in a relational data base, EMPRESS. In addition, the object-oriented spatial data is mapped to a proprietary EMPRESS kernel within the SYSTEM 9 executable. This means that while the data model appears objectoriented to the user, there is a behind-the-scenes translation going on between the relational data base and the spatial data model. We found this unwanted overhead to be overly cumbersome and detrimental to the performance required of a multidimensional spatial analysis and visualization system. This overhead is present to some degree in all commercial off-the-shelf geographic information systems products.

Having discovered that commercial-off-the-shelf geographic information systems were not going to provide the performance we had hoped for, we came up with an alternative solution. We determined that we needed complete control over the data management in order to optimize for speed. Additionally, we needed to implement the system in an environment that supports a high level of modularity. Realizing that part of the overhead involved in using commercial-offthe-shelf geographic information systems is taken up by large code segments supporting analytical and operational functionality that is rarely used or needed for many applications, we decided that PNLdeveloped analytic and operational modules would greatly streamline throughput. This approach allows
PNL to add more value and permits easy customization for specific applications.

The approach we decided on was to integrate the Application Visualization System with the POET $\mathrm{C}++$ object-oriented data management class library. By establishing a direct link between the Application Visualization System geometry and the object data base, we are able to provide rapid interactive object-oriented data retrieval and queries in a compiled environment. This approach allows us to develop in both the " $\mathrm{C}$ " and " $\mathrm{C}++$ " languages in order to allow us to optimize for speed, extensibility, and data abstraction. Analysis modules are developed in $\mathrm{C}++$ so that objects contained in base class code can be inherited by future application modules. In some cases, the data structure required by the Application Visualization System is not in a format the analyst is comfortable with. Data abstraction allows a class to be constructed which communicates with the analyst in his or her terms in the foreground while communicating in Application Visualization System data structures in the background.

This solution allows for excellent performance, unlimited extensibility, and applicability. It should also give PNL a technological advantage in time-space analytic applications. While the most recent release of the Application Visualization System (AVS 5.0) supports $\mathrm{C}++$, prior releases have not. As a result, this system would not have been feasible even several months ago.

The progress to date has produced a one-way data retrieval path of the Application Visualization System object's attribute data from the object data base by slicing a three-dimensional volume and projecting the slice orthogonally onto a two-dimensional plane (window). A user can then interactively select a point on the two-dimensional projection and obtain data from the object-oriented data base that is associated with the geometric object. The fourth dimension (time) is/will be handled by either sweeping the cut plane through a volume (two-dimensional crosssections are then animated to show the temporal component), or generating an animation via class member functions returned from a data base query. 
The next version will permit a user to select data from the data base and then create a visualization based on the data retrieval (Query-Based Animation).

Initial funding has allowed us to rule out the integration of commercial-off-the-shelf geographic information systems with the Application Visualization System for volumetric time-dependent analysis and provided us with the insight to design the current system. To present, our work has concentrated on the geometric side of the system and we have succeeded in getting a plane-cutting ${ }^{(a)}$ module to work, as well as integrating " $\mathrm{C}$ " and " $\mathrm{C}++$ " code modules within Application Visualization System. Additionally, we have succeeded in establishing an intelligent link between Application Visualization System geometric objects and persistent classes. The link has been implemented at the socket level using a client-server architecture. This is demonstrable as a one-way interactive data selector via the two-dimensional cut

(a) Affine mappings from three- and four-dimensional spacetime to tro-dimensions (monitor screen or hard-copy output device) is performed utilizing the plane-cutting and ray-stabbing tochniques of computational geometry. plans selected from a three-dimensional volume. We are currently able to animate the cut plane and actually select a point in the volume as the plane sweeps through. The system returns information on the selected point nearly instantly as it is selected.

We anticipate performance problems as the models being analyzed become large. This will be due to the single-processor limitation we have with the current platform (SUN SPARC stations). Improved performance will come from parallelizing many of the geometric and data base operations. Although we have successfully ported the Application Visualization System client to the STARDENT, overall performance is best when executed from the SUN platform. A port to the Silicon Graphics/4 platform is the most logical. Both Application Visualization System and POET offer SGI C++ versions.

\section{Presentations}

4-DIVAS was presented at the Second International Conference on GIS in Environmental Modeling in Breckenridge, Colorado at the ond of September. An additional presentation will be made during the first week of November at the GIS/LIS '93 conference in Minneapolis. 


\title{
Spectroscopy Techniques for Analysis of DNA Damage
}

\author{
A. F. Fuciarelli (Biology and Chemistry)
}

Exposure of DNA to free radical-generating agents, such as ionizing radiation and selected chemicals, results in a multiplicity of molecular damage that is also characterized in terms of the spatial distribution along the DNA molecule. We are using specific DNA products as molecular probes of nucleic acid damage in an effort to increase our understanding of the mechanisms underlying free radical damage to cells and the impact of these lesions on biochemical processes.

Oligonucleotide synthesis techniques were used to prepare DNA substrates of defined base composition for irradiations. Mass spectrometry methodology was used for product analysis to assess: 1) electron migration along DNA as a potential mechanism by which radiation-induced damage can be manifested distal to the sites of initial energy deposition in DNA, 2) the influence of DNA base sequence and DNA structure on the distribution of free radical-induced molecular products, and 3) the influence of the microenvironment on product distribution in irradiated solutions of DNA. Our efforts will provide relevant data regarding the contribution of electron transfer along DNA to formation of locally multiply damaged sites created in DNA by exposure to ionizing radiation. Efforts continued with regards to assessment of radiation-induced perturbations of DNA structure and function by incorporating modified DNA products into synthetic oligonucleotides to provide substrates for advanced molecular and analytical studies. This portion of the project involves application of our capabilities in synthetic DNA chemistry techniques in an effort to provide our laboratory, and our colleagues in the MSRC, with substrates for study. In addition, we also continued to develop sample handling techniques and mass spectrometric methodology which will ultimately permit studies addressing the formation, repairability, and repair kinetics of specific molecular lesions in DNA extracted from eukaryotic cells exposed in vivo to free radicalgenerating agents. These studies will contribute to the knowledge base regarding DNA damage induction to complement studies in repair, mutagenesis, transformation, and cell death.

\section{Electron Migration in DNA}

Electron migration along DNA is a mechanism by which radiation-induced damage can be manifested distal to the initial point of energy deposition in DNA and is an important contributing mechanism to formation of locally multiply damaged sites in DNA. We developed a model system using 5-bromouracil $(\mathrm{BrU})$ synthetically incorporated into oligonucleotides to study electron migration in aqueous solutions of DNA following exposure to ionizing radiation. We demonstrated that

1. electron migration occurred in aqueous solutions of DNA and that differences in the extent of migration occurred as a function of DNA strandedness and base sequence

2. electrons were capable of migrating up to 7 base pairs in a segment of DNA containing guanine bases

3. electrons were capable of preferential migration from the $5^{\circ}$ to $3^{\circ}$ direction in a segment containing guanine bases, but the degree to which electrons moved in this direction was less in a segment containing adenine bases

4. the hydrogen atom captured by the uracilyl radical in a solution of bromouracil is captured from t-butanol (placed in solution to scavenge hydroxyl radicals resulting from water radiolysis), whereas, in $\mathrm{BrU}$-containing oligonucleotides, the hydrogen atom is captured from the $2^{\circ}$ carbon of the adjacent base in the DNA molecule; an observation which has important implications with regards to formation of DNA strand breaks.

Our continuing efforts in this area will focus on a more complete understanding of the mechanisms by 
which electron migration occurs in irradiated solutions of DNA in an effort to gain an understanding of how radiation-induced damage can be manifested distal to the site of initial energy deposition. We will work toward use of biologically relevant sequences of DNA such as those found in promoter regions and regions of DNA that bind proteins.

\section{Synthesis of DNA Fragments Containing Modified DNA Products for Studies of Structure and/or Function Relationships in DNA Exposed to Ionizing Radiation}

Our group has developed synthetic methods to generate modified DNA products identical to those introduced following exposure to ionizing radiation for use as authentic standards for mass spectrometry. We are using this experience to create model DNA systems for use in multidisciplinary studies that will examine the role of DNA lesions in structure and/or function relationships. Our involvement in studies utilizing $8,5^{\circ}$-cyclodeoxynucleotides was an effort to place our group in the forefront of this area of research. Unfortunately, incorporation of these uniquely significant lesions into oligonucleotides for nuclear magnetic resonance or molecular biology study requires a substantial effort in organic chemistry. To establish credibility in this area of research, we have modified our efforts on this project in an attempt to utilize previously published synthetic protocols to generate DNA products for structural studies. In this regard we worked with E. Schroeder (who visited our laboratory for 6 weeks) and H. Box at the University of Buffalo to directly incorporate a deoxyribosylformylamine fragment into oligonucleotides. Our second effort in this area involved a collaboration with Dr. M. Kennedy (MSRC) in which we have initiated synthesis of a DNA fragment containing thymine glycol by chemical modification of oligonucleotides. Successful preparation of these substrates will permit nuclear magnetic resonance, mass spectrometry, and enzymatic studies using oligonucleotides of defined DNA base composition in an effort to further address the structure and/or function relationship of lesions formed following exposure to ionizing radiation.

\section{Radiation-Induced DNA-Protein Cross-Linked Species}

In collaboration with D. L. Springer and C. G. Edmonds, radiation-induced cross-links between histone H2A and thymine were detected using electrospray-mass spectrometry methodology demonstrating that this technology could be profitably applied to analysis of DNA-protein cross-link analysis following exposure to ionizing radiation. Our efforts in this area led to the detection of radiation-induced cross-links between DNA and proteins at doses as low as $1 \mathrm{~Gy}$. This work is important both for the further understanding of radiation-induced damage in cells and for the potential use of DNA-protein cross links as markers of radiation exposure.

\section{Free Radical-Induced DNA Base Products in Solutions Exposed to Ultrasound}

In collaboration with D. L. Miller (Life Sciences Center), we have examined DNA product formation following exposure to ultrasonic cavitation. DNA damage was evaluated by measuring the formation of purine and pyrimidine products following exposure of DNA solutions to ultrasonic cavitation using combined gas chromatography-mass spectrometry. Hydrogen peroxide yields were measured after each exposure to quantify the cavitation activity. Purine and pyrimidine products identified were those typically observed following exposure of DNA to hydroxyl radicalgenerating systems such as ionizing radiation, hypoxanthine/xanthine oxidase or hydrogen peroxide in the presence of transition metal ions. The yields of these products were directly correlated with cavitation activity as measured by residual hydrogen peroxide concentrations and increased in the following order: thymine glycol $\sim$ cytosine glycol $>8$-oxoAde $>$ FAPyAde 5-HMU 5,6-diOHCyt > FAPyGua. Unexpectedly, 8-oxoguanine did not exhibit a dosedependent increase above background levels and this observation is inconsistent with processes involving metal-ion dependent formation of hydroxyl radicals from hydrogen peroxide. In addition, the product yields were far too large to result from the residual hydrogen peroxide. Thus, ultrasonic cavitation appears to have a mode of action distinct from either ionizing radiation or formation of hydroxyl radicals via Fenton-like reaction with transition metals.

This work provides an increased appreciation of the mechanism leading to free radical damage in cells ranging from singly damaged sites on DNA that are easily repaired by enzymatic processes to multiply damaged sites, such as those generated by exposure to ionizing radiation, which demand a significantly more complex form of enzymatic processing for damage repair. 


\section{Publications}

Beach, C., A. F. Fuciarelli, and J. D. Zimbrick. "Electron Migration Along 5-bromouracil-substituted DNA Irradiated in Solution and in Cells." Radiation Research, in press.

Edmonds, C. G., M. S. Weir, A. F. Fuciarelli, B. D. Thrall, and D. L. Springer. "Electrospray Ionization Mass Spectrometry and Tandem Mass Spectrometry of Chick Erythrocyte Histones." Rapid Communications in Mass Spectrometry, in press.

Fuciarelli, A. F., E. C. Sisk, and J. D. Zimbrick. "Electron Migration in Gamma-irradiated Solutions of DNA. International Journal of Radiation Biology, in press.

\section{Presentations}

Fuciarelli, A. F., E. C. Sisk, L. E. Matson, K. Miaskiewicz, J. H. Miller, and J. D. Zimbrick. 1993. "Electron Migration in Gamma-irradiated Solutions of DNA." Presented at the conference on DNA Damage: Effects on DNA Structure and Protein Recognition, Burlington, Vermont.

Fuciarelli, A. F., E. C. Sisk, L. E. Matson, K. Miaskiewicz, J. H. Miller, and J. D. Zimbrick. 1993. "Electron Migration in Gamma-Irradiated Solutions of DNA." Presented at the meeting entilled, "DNA Damage: Effects on DNA Structure and Protein Recognition (New York Academy of Sciences), Burlington, Vermont.

Fuciarelli, A. F., E. C. Sisk, J. H. Miller, and J. D. Zimbrick. 1993. "Electron Migration in Gamma-irradiated Solutions of DNA." Presented at the Biophysical Society Meeting, Washington, D.C.
Fuciarelli, A. F., E. C. Sisk, J. H. Miller, and J. D. Zimbrick. 1993. "Electron Migration in Gamma-irradiated Solutions of DNA." Presented at the Forty First Annual Meeting of the Radiation Research Society.

Maiskiewicz, K., J. H. Miller, R. L. Ornstein, A. F. Fuciarelli, J. D. Zimbrick, and J. A. Raleigh. 1993. "DNA Intramolecular Crosslinks: Theory and Experiment." Presented at the Forty First Annual Meeting of the Radiation Research Society.

Weir, M. S, D. L. Springer, A. F. Fuciarelli, B. D. Thrall, and C. G. Edmonds. 1993. "Characterization of Natural and Radiation Induced Modifications of Histones." Presented at the 7th Symposium of the Protein Society, San Diego, California.

\section{Invited Plenary Session}

Fuciarelli, A. F., E. C. Sisk, J. H. Miller, and J. D.

Zimbrick. 1993. "Electron Migration in Gamma-irradiated Solutions of DNA." Forty First Annual Meeting of the Radiation Research Society. March 20-25, 1993.

\section{Participation in Scientific Review}

Study Section: National Institutes of Health: Regulation, Function and Specificity of Proteins Involved in Mammalian Cells Exposed to Ionizing Radiation. Conducted in Washington D.C., July 22-23, 1993. 


\section{Structural and Kinetic Studies at Model Oxide Surfaces}

S. A. Joyce and D. B. Kay (Chemical Structure and Dynamics)

The objective of this project was to examine phenomena occurring on model oxide surfaces in an effort to unravel the mechanistic details of the complex interfacial chemistry occurring in the subsurface environment. Scanning probe microscopies (scanning tunneling microscopy and atomic force microscopy) were employed to study the growth and structure of model oxide surfaces. Molecular beam scattering and surface analytical techniques were utilized to explore the dynamics and kinetics of adsorbates interacting with these surfaces.

Many of the solid surfaces of interest to the environmental restoration of the soils and groundwaters on the Hanford Site are insulators in their bulk forms and thus not amenable to study with conventional electronbased surface analytical techniques. The growth of microscopically thin films on conducting substrates overcomes the electrical charging problems associated with bulk insulators. Scanning tunneling microscopy studies of thin film magnesium oxide, $\mathrm{MgO}$, grown on a molybdenum substrate were begun in FY 1993. Topographic images of a 2- to 3-layer thick film of $\mathrm{MgO}$ were obtained, indicating the feasibility of the thin film approach. Atomically-resolved images of the film were not obtained, most likely due to the inherent roughness of the underlying molybdenum substrate. Efforts to obtain flatter substrates are under way. In related experiments, we have synthesized thin films of brucite, $\mathrm{Mg}(\mathrm{OH})_{2}$, by exposing a $\mathrm{Ru}(0001)$ surface to molecular beams of $\mathrm{H}_{2} \mathrm{O}$ and $\mathrm{Mg}$. The basal plane of $\mathrm{Ru}$ was chosen as the substrate due to its favorable crystal structure for growing epitaxial Mg. Real-time mass spectrometry was used to monitor the evolution of molecular hydrogen from the highly exothermic reaction of $\mathrm{Mg}$ and $\mathrm{H}_{2} \mathrm{O}$. Subsequent heating of the $\mathrm{Mg}(\mathrm{OH})_{2}$ thin film yielded $\mathrm{H}_{2} \mathrm{O}$ vapor from the decomposition of brucite to periclase $(\mathrm{MgO})$. Future studies will focus on unraveling the chemisorption of $\mathrm{H}_{2} \mathrm{O}$ and halocarbons on these model mineral surfaces. 


\title{
Structural Studies of Modified Histone Species
}

\author{
D. L. Springer (Biology and Chemistry) and C. G. Edmonds (Chemical Sciences)
}

It is our premise that a fundamental understanding of the relationship of histone modification to chromatin structure and its alteration through cell cycle or cell damage and repair can only be obtained by consideration of the full range of type, extent, and site of modifications. We hypothesize that the normal time-dependent profile of histone modifications through the cell cycle is non-random and predictable. Furthermore, this consistency will also pertain in the course of modifications arising from response to chemical or radiation insult. In these experiments, we evaluated the similarities and differences in profile that arise in the normal cycle in cultured human cells and the differences which arise in the response to chemical insult using electrospray ionization mass spectrometry and tandem mass spectrometry.

Histones, which together with other specialized protein and DNA form the extraordinarily complex structure of chromatin, are post-translationally modified by acetylation, methylation, phosphorylation, mono- and poly(ADP)ribosylation, glycosylation, and ubiquitination. Each of these modifications is known to have important associations with alterations in chromatin structure which occur throughout the cell cycle or in response to chemical- or radiation-induced damage. The structural biology of these processes is a complex picture of protein-protein and protein-DNA associations modulated, in part, by protein posttranslational modification. The full details of the changes in level of all of these modifications remain to be elucidated. We hope to demonstrate the feasibility of our ultimate goal to elucidate the full details of these processes. We hypothesize that there are histone modifications, which are sensitive and predictive and that distinguish natural and chemical or radiation induced alterations in chromatin structure.

To test this hypothesis we are carrying out experiments which will ultimately permit the evaluation of the profile of post-translationally modified histones during the cell cycle and identify perturbations of this profile of modifications due to insult from chemical agents, radiation, or their combination. For these studies, human cells grown in culture will be separated according to their stage in the cell cycle using flow cytometry. Histones will be isolated and purified by high performance liquid chromatograph and polyacrylamide gel electrophoresis and the extent and types of modifications determined by electrospray ionization mass spectrometry and tandem mass spectrometry. Additionally, experiments intended to demonstrate the time-courses of modification, including their sequence location, will be undertaken. This will be accomplished by the examination of histones from cells in various stages of the cell cycle that have been isolated by flow cytometry. Further, specific information about the type and location of modifications will be obtained by combined liquid chromatography-tandem electrospray ionization mass spectrometry of peptides obtained by selective proteolysis with Staphylococas aureus (V8) protease or trypsin. These techniques will permit, for the first time, direct analysis with high sensitivity and unambiguous evaluation of the nature and extent of multiple modification of histones as a function of cell cycle. In addition, subsequent experimentation may identify modification changes in response to chemical and/or radiation insult. Results from this work will provide, for the first time, information on several types of histone modifications simultaneously as a function of the position in the cell cycle, and will contribute to our understanding of regulatory processes governing cellular replication.

We have developed methods for the evaluation of the range of histone modifications based on biochemical and mass spectrometric techniques. For this, nuclear histones are isolated from chick erythrocytes and from exponentially growing cultured K562 cells, a human erythroleukemia-derived cell line. The histones are isolated from nuclei preparations, purified by reversed phase high performance liquid chromotograph and the intact protein evaluated for post-translational modificztion by gel electrophoresis and electrospray ionization mass spectrometry. The alterations in the extent and pattern of modification have been evaluated by experiments on histones isolated from cultured 
cells exposed to sodium butyrate, a deacetylase inhibitor that causes significant shifts in the degree of histone acetylation.

Early success in the isolation and characterization of the post-translational modifications, which could be assigned as combinations of methylation and acetylation, have been repeated on smaller samples. Purification and analysis of histones derived from as few as $10^{6}$ cells has been demonstrated. Refinement of the methods to permit the analysis of still smaller numbers of cells obtained by fractionation of cell cycle populations by flow cytometry is continuing. We observe in batches of chick erythrocytes obtained from different suppliers masses for histone H2B, which sugsest that the protein is substatitially uumodified and which differ from one another by a value of 26 daltons. This difference is not readily assigned to a simple post-translational modification and we hypothesize that these proteins may differ from one another by some unrecognized clonal difference in the respective genes. Peptide mapping experiments to test this hypothesis and to identify the nature of this variation in primary sequence are in progress. In human $\mathrm{K562}$ cells we found that H2B is substantially unmodified. In this case, three of the known sequence variants of this protein are distinguishable in the electrospray ionization mass spectrum. Fragmentation of this intact protein in the electrospray atmosphere-vacuum interface has been demonstrated affording numerous cleavages which are assignable on the basis of the known sequence of the protein. The fragment ions produced include prominent responses for the $\mathrm{N}$-terminal 50 residues of the protein bounded by a proline residue. This peptide fragment, containing two other proline residues, covers the majority of the region shown to be modified in published studies. This experiment suggests that a rapid and sensitive mass spectrometric mapping experiment for the modifications occurring in the $\mathrm{N}$-terminal region of this and other histone species will be practical. The isolation and characterization of the histones in human diploid fibroblasts grown in culture has been undertaken in collaboration with $\mathrm{Dr}$. Michael Smerdon, Department of Biophysics and Biochemistry, Washington State University, Pullman. Correlations in the nature and extent of modification of the histones occurring in this cell line as compared to those in the very rapidly proliferating $\mathrm{K} 562$ are consistent with the substantially lower rate of growth. On this basis this cell line appears to be a more realistic model for the patterns of post-translational modifications nccurring in normal tissues.
As part of our responsibility as a member of the larser scientific community, we have continued to cultivate interactions with university- and industrybased colleagues. We have undertaken electrospray ionization mass spectrometry experiments which, in some cases address questions, while not strictly relevant to this research effort, that have permitted us to continue to develop and test the wider applicability of our methods. We have taken the opportunity to test the validity of our ideas and the quality of our accomplishments in the submission of a proposal entitled, "Core Histone Modification in Perturbed Chromatin." The comments of the reviewers have had a strong and beneficial influence on our thinking and our work. A resubmission of this proposal has been prepared and will be submitted to DOE at the beginning, of FY 1994.

\section{Publications}

Alam, S. L., J. D. Satterice, and C. G. Edmonds. "Complete Amino Acid Sequence of the Glycera dibranchiata Monomer Hemoglobin Component IV. Structural Implications." Protein Chemistry, submitted.

Edmonds, C. G., J. A. Loo, R. D. Smith, A. F. Fuciarelli, B. D. Thrall, J. E. Morris, and D. L. Springer. 1993. "Evaluation of Histone Sequence and Modifications by Electrospray Ionization Mass Spectrometry and Tandem Mass Spectrometry." Journal of Taxicology and Environmental Health 40:159.

Loo, J. A., R. R. Ogorzalek-Loo, D. R. Goodlett, R. D. Smith, A. F. Fuciarelli, D. L. Springer, B. D. Thrall, and C. G. Edmonds. 1992. "Elucidation of Covalent and Noncovalent Association of Proteins by Electrospray Ionization Mass Spectrometry." Techniques in Protein Chemistry :, ed. R. H. Angellette, Academic Press.

Loo, J. A., M. M. Siegel, J. Huang, B. Lin, R. Trao, and C. G. Edmonds. "Structures of Bacitracin A and Isolated Cogeners: Sequencing of Cyclic Peptides with Blocked Linear Sidechains by Electro-spray Ionization Mass Spectrometry." Biological Mass Spectrometry, submitted.

Weir, M. S., D. L. Springer, A. F. Fuciarelli, B. D. Thrall, and C. G. Edmonds. "Characterization of Natural and Radiation Induced Modifications of Histones." Techniques in Protein Chemistry, 5, Academic Press, in press.

\section{Presentations}

Edmonds, C. G., J. A. Loo, R. D. Smith, A. F. Fuciarelli, B. D. Thrall, J. E. Morris, and D. L. Springer. 1992. "Evaluation of Histone Sequence and Modifications by Electrospray Ionization Mass Spectrometry and Tandem Mass Spectrometry." Presented at the Hanford Life Sciences Symposium, Richland, Washington. 
Edmonds, C. G., M. S. Weir, B. D. Thrall, J. D. Morris, and D. L. Springer. 1993. "Evaluation of Variable Posttranslation Modification of Human Histones by Electrospray Ionization Mass Spectrometry and Tandem Mass Spectrometry." Presented at the 41th ASMS Conference on Mass

Spectrometry and Allied Topics, San Francisco, California. 


\section{Synchrotron Research/Advanced Photon Source Beamline}

B. R. Stults (Molecular Science Research)

The objective of this research is to apply synchrotron radiation to the study of complex environmental systems. To achieve our objectives, we have initiated research projects using existing synchrotron light sources and have initiated efforts to design specialized facilities to take advantage of the special capabilities being built at the Advanced Photon Source.

In FY 1993, we continued our program to introduce the unique research capabilities afforded by the use of synchrotron radiation for application to DOE environmental problems. Our efforts were focused in two major areas:

1. The development of conceptual designs for special synchrotron radiation beamlines at the Advanced Photon Source (APS) being constructed at Argonne National Laboratory.

2. The initiation of research projects at existing synchrotron radiation facilities, e.g., National Synchrotron Radiation Laboratory and the Stanford Synchrotron Radiation Laboratory.

Key to all of our activities has been collaborative interaction with nembers of the Pacific Northwest Consortium (PNC) for synchrotron radiation research, which has active membership of over $\mathbf{5 0}$ scientists from PNL, the University of Washington, Washington State University, the University of Oregon, Oregon State University, and Boeing Company.

The PNC-Collaborative Access Team (PNC-CAT) completed conceptual designs for three beamlines to be built at the advanced photon source. The conceptual designs were approved by the advanced photon source review board. A proposal currently under review was submitted to the DOE for capital funding (approximately $\$ 6.0 \mathrm{M}$ ) for construction of the new advanced research facilities at the advanced photon source.
A major component of our advanced photon source beamlines will be our ability to focus $\mathrm{x}$-rays to extremely small regions. We have done careful computer simulations of the characteristics of a new $x$-ray focusing device that we have proposed, which compresses the $X$-rays to a small spot as they pass through a tapering capillary. Our computer simulations verify that the capillary compression produces a smaller and more intense $\mathrm{x}$-ray spot than the conventional mirror focusing.

In collaboration with members of the PNC, several research projects at PNL were initiated during FY 1993:

- study of the local bond structures of $\mathrm{Ce}$ in a series of Ce-doped alkali silicate glasses

- analysis of the structure of $\left(\mathrm{Yb}_{0.2} \mathrm{Z}_{0.8-\mathrm{x}} \mathrm{Ce}_{\mathrm{x}}\right) \mathrm{O}_{2.9}$ compounds

- study to determine the applicability of synchrotron radiation for analysis of tank waste, in particular, the speciation of $\mathrm{Sr}$ in simulated tank waste

- analysis of the phases present in rapidly deposited, nanosized $\mathrm{ZrO}_{2}$ and $\mathrm{Fe}$ oxides

- measurements of the bonding geometries of $\mathrm{Cr}(\mathrm{VI})$ absorbed or hydrotalcites which are being considered for in situ remediation at the Hanford Site.

A data analysis system was developed at PNL utilizing the data analysis software from the group at the University of Washington. The data analysis system has also been installed on a portable computer system for preliminary analysis of data during the actual data collection process. This on-line analysis enables the rejection of questionable data and increases the erficiency of time spent on an offsite synchrotron light source. 


\section{Publication}

"A Capillary Concentrator for an X-ray Microprobe" has been submitted to Nuclear Instruments and Methods for publication.

\section{Presentation}

An invited paper on capillary $x$-ray focusing was presented at the Synchrotron Radiation Instrumentation Conference held last August at Argonne National Laboratory. 


\title{
Technological Response
}

\author{
W. B. Ashton (Technology Management Planning)
}

During FY 1993, the technology response area of the PNL Global Studies Program continued a series of studies and computer model developments to improve our understanding of how longterm structural changes driven by technological progress can be expected to affect global environmental processes. The focus of the technology response work is on "integrating " aspects of technological change, not simply investigating particular technology "fixes" to limit adverse environmental impacts. The results of this work will be organized into computational models suitable for incorporation into the Second Generation Model at PNL.

In FY 1993, the technology response work was organized into three main application areas 1) modeling technology impacts on natural global cycles, 2) modeling carbon sequestration, and 3) understanding longrun technological change.

\section{Technology Impacts on Natural Global Cycles}

\section{A System Dynamics Model of Technolngy in the} Global Carbon Cycle-The PNL Second Generation Model will ultimately require a model of the biosphere which includes a global representation of the carbon cycle. This project will produce such a model by adapting the original Goudriaan and Ketner (1984) biosphere carbon-cycling model to a regional geographical basis referenced to the 20 geo-political regions of the Second Generation Model.

In FY 1993, the computer software code from FY 1992 LDRD work was corrected; the format of the user input-output interaction with the computer software was improved; the $\mathrm{CO}_{2}$ input mode wo be externally supplied by the user rather than calculated was changed; the global sum of the 20 regions' output against the original Goudriaan-Ketner model output from the IMAGE 1.0 version was validated; and model test-runs consistent with PNL-Richland Technology Planning and Analysis Center work on the GCAM project were completed.
A review of the model's performance and linkages with the Second Generation Model are now being undertaken in preparation for decisions regarding future work.

Tangible output from this work includes a revised computer program for the Second Generation Modelregionalized model on magnetic disk (PC-DOS), and a working paper documentation of the work achievements. The computer code was revised and modelgenerated results were produced to test performance of the revised code.

\section{A Review of the Emissions and Effects of Trace} Metals-The purpose of this report is to describe the current state of knowledge regarding the global sources and quantities of heavy metal emissions, their transport and fate, their potential health and environmental effects, and strategies to control them. Various studies of these materials have been undertaken under local or regional conditions, but no one has ever undertaken an assessment on a global scale. The approach for this work is to review the literature on this topic and to consult with experts in the field. Ongoing research activities and research needs are discussed.

This work concluded that heavy metal pollution is an issue that warrants serious attention. Although estimates of annual heavy metal emissions and historical deposition patterns are crude, it appears that heavy metal emissions are increasing and that anthropogenic sources are the main contributor to this increase. For example, although mercury use in paints and batteries is on the decline, mercury and other heavy metal emissions from coal combustion are expected to rise in the future as coal consumption increases. Heavy metal pollution will also increase in developing countries as the demand for goods and services incresses.

In order for effective strategies for combating heavy metal pollution to be devised, the uncertainties regarding the global cycling of heavy metals need to be addressed. Further research is needed. To understand the source and fate of heavy metals, a holistic approach considering all environmental media must be used. 


\section{Carbon Sequestration}

The Potential for Sequestration of Carbon Dioxide in Industrial Chemicals and Polymers-This work explores the concept of generating polymers composed mainly of carbon dioxide combined with another organic moiety, such as the polycarbonates (used in plastic bottles), the polyurethanes (foams and coatings), and the polyureas (specialty uses). These polymers hold the promise of serving as a long-term storage medium for carbon from combustion-generated carbon dioxide emissions.

In FY 1993, an assessment of the potential for these materials was completed. The findings indicate that considerable commercial effort has been expended in the last 10 years to use $\mathrm{CO}_{2}$ as a feed stock for specific polymers. In particular, $\mathrm{CO}_{2}$ is now being used as a substitute for phosgene in the preparation of a great number of polycarbonates. This polymer production requires the use of a catalyst in the presence of an epoxide and $\mathrm{CO}_{2}$. Polyureas and polyurethanes are also being investigated on large scale, but it appears that only polycarbonate production is currently utilizing $\mathrm{CO}_{2}$ on a commercial scale. Numerous other polymer syntheses have been investigated at the research level.

Also during FY 1993, contacts with the University of Washington and Massachusetts Institute of Technology were made to discuss collaboration with PNL in the development of plastic materials suitable for foaming.

PNL concepts regarding carbon dioxide-based polymers were also discussed in detzil with Battelle Columbus Laboratory biomass researchers, particularly Dr. Ed Lipinsky, and the approaches to investigate a hypothetical entity, poly(carbon dioxide), for a collaborative effort with the Environmental and Molecular Sciences Laboratory were discussed.

\section{A Supply Curve for Carbon Capture/Sequestration for U.S. Utilities-This work involves development of a U.S. national " $\mathrm{CO}_{2}$ sequestration supply curve" that identifies the amount of $\mathrm{CO}_{2}$ that can be controlled as a function of the cost per unit of $\mathrm{CO}_{2}$ controlled. The case study approach is used to develop the cost esti- mates for gasification combined-cycle plants.}

During FY 1993, a report was written containing cost estimates for sequestration of carbon via scrubbing as a function of transportation distance to storage sites from over 300 hypothetical utility power plants locations in the U.S. (over 800 gasification combinedcycle units). The principle findings are the cost results, which indicate that the cost of $\mathrm{CO}_{2}$ recovery can be much lower than expected. Levelized costs in real 1990 dollars of $\$ 0.0631 / \mathrm{kWh}$ (for a base-case gasification combined-cycle plant with no $\mathrm{CO}_{2}$ recovery), $\$ 0.0863 / \mathrm{kWh}$ to $\$ 0.1265 / \mathrm{kWh}$ (for ocean disposal at $500 \mathrm{~m}$ ), and $\$ 0.0850 / \mathrm{kWh}$ to $\$ 0.1430 / \mathrm{kWh}$ (for depleted oil/gas well disposal) were derived.

This information will be used along with similar information on other $\mathrm{CO}_{2}$ control options (e.g., carbon taxes, conservation, etc.) to help evaluate the best mix of policy options.

\section{Modeling Long Run Technological Change}

Structural Change-This work is designed to survey what is known about the role of structural change as a determinant in energy use and environmental impacts. It has been established that structural change in the form of changes in industrial output and consumer value shifts has been a major factor in explaining energy investment and fuel use decisions. However, very little is known about structural change processes and even less is known regarding how to model this phenomenon in the Second Generation Model environment.

In FY 1993, economic literature was reviewed and the research needed to better understand structural change was identified. In addition, a new concept for analysis and modeling of structural change was developed to take into account the decision logic of the agenis allocating resources in the economy, but normalized for changes in the size of the economy. For the final demand portion of the economy, structural change is considered to be any change in expenditure patterns after normalizing for the level of expenditure from one period to another. A rationale for this definition was established and research needed to investigate the implications of this conception was identified.

Long-Run Technological Change in the Electric Power Industry-This project investigated methods to model long-run technological change (disembodied and embodied technical change) in the CES productions functions used in the Second Generation Model. The work consisted of a literature review, development of modeling concepts for adjusting the CES technology parameters, and examination of the concepts in a case 
study of coal-fired, electric generation technologies to evaluate various modeling concepts.

In FY 1993, literature was obtained and reviewed to understand basic modeling approaches used for representing technical change in various industries. New concepts for the processes of technical change based on evolutionary biology models were identified and documented. In addition, basic data from the Edison Electric Institute on long-run technological change in coal-fired steam electric generation was collected in preparation for testing alternative models of long run change.

\section{A Review of Literature on Technological Change and Productivity in Manufacturing and Agriculture- Literature and data collection for this review was completed in FY 1993 and a working paper sum- marizing the results has been drafted.}

\section{Publications}

Brown, D. 1993. "The Costs of Carbon Dioxide Control for Gasification Combined-Cycle Plants in the United States." Energy-The International Journal, in press.

Brown, D. et al. 1993. Carbon Diaxide Control Costs for Gasification Combined-Cycle Plants in the United States. PNL-SA-22634. Globul Studies Program, Pacific Northwest Laboratory, Richland, Washington.

Diamond, C., and W. B. Ashton. 1993. "A Review of R\&D, Technology and Productivity in Agriculture." PNL Working Paper, Global Studies Program. Pacific Northwest Laboratory, Richland, Washington.

Friedman, J., and W. B. Ashton. 1993. "The Effects of R\&D and Technology on Total Factor Prodiletivity Growth." PNL Working Paper, Global Studies Program, Pacific Northwest Laboratory, Richland, Washington.
Friedman, J. R. et al. 1993. A Review of the Global Emissions, Transport, and Effects of Heavy Metals on the Environment. PNL-SA-22394. Global Studies Program, Pacific Northwest Laboratory, Richland, Washington.

Kavanaugh, D. et al. 1993. "Modeling and Estimation of Long-Run Technological Change in Electric Power: The Case of Coal-Fired Steam Generation." PNL Working Paper, Global Studies Program, Pacific Northwest Laboratory, Richland, Washington.

Kickert, R. 1993. "1993 Report for: Development of 20-Region Terrestrial Biosphere Carbon Dynamics Simulation." PNL Working Paper, Global Studies Program, Pacific Northwest Laboratory, Richland, Washington.

Molton, P. M., and D. A. Nelson. 1993. Sequestration of Carbon Diaxide in Industrial Polymers: Building Materials for the 21st Century. PNL-SA-22528. Global Studies Program, Pacific Northwest Laboratory, Richland, Washington.

Roop, J. M. 1993. "Structural Change: Basic Concepts and a New Definition." PNL Working Paper, Global Studies Program, Pacific Northwest Laboratory, Richland, Washington.

Presentation

Kavanaugh, D. 1993. "Technical Progress in Renewable Energy Technologies." Presented at the International Association for Energy Economics, Seattle, Washington.

\section{Computer Code}

Kickert, R. 1993. LDRD 1993 Revision of Terrestrial Biosphere C-Cycling Model. PC-Computer Code, Global Studies Program. Pacific Northwest Laboratory, Richland, Washington.

\section{Other Accomplishments}

This project provided support during the summer of 1993 for Jennifer Friedman, a PNL staff member on educational leave. 


\title{
The Technical Basis for Logit Analysis Applied to Technology Markets
}

\author{
W. B. Ashton (Technology Management Planning)
}

This project involved an investigation of the technical basis for using the logit function to estimate market penetration rates for new technologies. Logit analysis, a widely used tool to model market shares, is used in the PNL Second Generation Model (SGM) of greenhouse gas emissions as the method to determine the penetration rate of new technologies and their eventual market shares. However, the foundations of logit analysis and the applicability of these foundations to the Second Generation Model modeling environment are not well known. This project will investigate the foundations of logit analysis and a variety of relevant applications to assess the applicability of the theory behind logit functions to regional technology penetration/diffusion modeling.

In FY 1993, this project focused on reviewing two aspects of the technical literature regarding use of the logit function for modeling market penetration of advanced technologies.

\section{Logit Functions}

The theoretical foundations and basic assumptions for the logit function, as well as the strengths and weaknesses of logit analysis in various modeling applications, were examined. The most prominent application studied was transportation modal split analysis. This investigation indicates that use of the logit function in the Second Generation Model modeling environment may be appropriate only for special circumstances which meet the mathematical assumptions of the logit model. The results of this analysis are documented in a PNL working paper.

These results will be compared against a new concept in market penetration modeling developed in the Global Studies Program in FY 1993 by J. Clarke. This concept is a model which represents the dynamic evolution over time of the probability distribution function for average cost of providing energy services. The relation of the logit function and dynamic cost probability distributions will be the target of future work.

\section{Market Barriers}

Technical literature on the barriers to penetration of new energy technologies was reviewed to identify those aspects of market penetration which might not be amenable to modeling with cost-based functions such as the logit. In FY 1993, literature emphasizing energy end-use technologies was obtained, reviewed, and documentation has begun.

Publication

Elson, A. 1993. "Use of the Logit Function for Modeling Technology Market Penetration." Working Paper. PNL Global Studies Program. Pacific Northwest Laboratory, Richland, Washington.

Other Accomplishments

This project provided support during the summer of 1993 for Robert Friedman, a PNL staff member on educational leave. 


\title{
Uncertainty Analysis for Computer Models
}

\author{
A. M. Liebetrau (Anatytic Sciences)
}

The purpose of this project was to continue development of practical methods of sensitivity and uncertainty analysis for complex computer codes that have a large number of input variables or extremely long run times. The methods are especially intended for system codes that involve the linking of several codes with these properties. The proposed work had two aspects: 1) to develop efficient sampling designs for simulation, and 2) to develop computationally tractable approximations to the (output) response surfaces of the models in question. Efficient input designs were used to reduce computing times by minimizing the number of runs required. The approximations were used to perform simulations that would otherwise be prohibitive because of the long run times involved. Essential properties of the approximation are that it preserve the essential features of the underlying code, and that it run much faster than its complex counterpart.

Human and earth systems are extremely complex processes. The modeling of these systems to assess the effects of climate change is an activity fraught with uncertainty. System models typically involve the linking of a series of computer codes, each of which is a detailed model of some physical or social process in its own right. In such system models, the output from one process model is the input to another. Traditional methods of uncertainty analysis are inadequate because of the sheer complexity of the modeling effort: Monte Carlo methods and the exhaustive evaluation of "what if?" scenarios to estimate sensitivities fail because of the heavy computational burden. This research was undertaken to develop more efficient methods for learning about and performing uncertainty analyses with system models that are constructed from a collection of computer codes.

Under this project, we have begun to develop a twotiered strategy for uncertainty analysis for systems of computer-intensive codes. The basic idea is to develop computationally less demanding approximations to the underlying codes that can be used for most uncertainty calculations. The three major elements of this strategy are 1) selection of an initial set of inputs at which to run the underlying code (the design step), 2) development of a suitable approximation to the response surface of the underlying code (the approximation step), and 3) the use of information from existing runs to determine the optimal locations for additional runs of the underlying code (the updating step).

Our research has shown that acceptable procedures exist for designing the set of initial runs of the underlying codes. Latin hypercube sampling is one widelyused design strategy that has proved very effective for sensitivity and uncertainty analysis. Consequently, we have used Latin hypercube sampling to select initial runs and have concentrated our efforts on the approximation step.

An initial step was to identify potentially useful strategies for approximating the response surface of the underlying code. Two methods were selected for further study. They involve the use of multivariate adaptive regression splines (the MARS procedure) and the use of regression trees (the TREES procedure). Both procedures can be described as constrained linear optimization procedures.

Both approximation methods were applied to two codes, the erosion productivity impact calculator (EPIC) code and the Analytic Repository Source-Term (AREST) code. The two codes are quite different, yet each has characteristics typical of codes used to simulate some system or process. The EPIC code was used in the Missouri, Iowa, Nebraska, and Kansas (MINK) study to simulate crop production for a variety of climate scenarios. The AREST code was developed to simulate the containment and release performance of a geologic repository for radioactive wastes. The response of the EPIC code is quite linear over regions of interest, but the output is truly stochastic. On the other hand, the AREST code is highly nonlinear but it can be run in a deterministic mode. The AREST code estimates releases of radionuclides, which of course must be non-negative. 
The MARS approximation procedure is attractive because it yields an approximation of minimal complexity (under specified constraints) for a given set of runs of the underlying code. A second desirable feature is that variance estimates computed from the MARS approximating surface were not significantly different than those computed directly from the underlying model. We discovered, however, that the MARS procedure did not work well when the response surface of the underlying model was constrained. Regression trees worked better for dealing with the "hard" zeros which are a unique feature of the AREST code (but typical of many physical or chemical processes). The results of this work suggest that a variety of approximation methods will be required to obtain suitable approximations to the many different codes that will likely be encountered in practice.

The expertise gained from the exercises described above is being tested and expanded in several applications. Multivariate adaptive regression spline approximations to the EPIC code have been used to test the results of the MINK study. Plans are under way to extend this methodology to provide an effects model for the entire United States. The results will also be used in the uncertainty analysis component of a performance assessment of the proposed high-level radioactive waste repository at Yucca Mountain, Nevada.

Publication

Liebetrau, A. M., P. D. Whitney, D. W. Engel, and C. A. LoPresti. "Computational Analogues to Complex ComputerBased Codes." Environmetrics, submitted.

Presentation

Scott, M. J., A. M. Liebetrau, and C. A. LoPresti. 1993. "Climate Uncertainty and the Regional Economic Impacts of Global Climate Change." Presented at the Thirty-Second Hanford Symposium on Health and the Environment in Richland, Washington.

Other Accomplishments

The funds for this project were used to support the participation of A. M. Liebetrau at the DOE-sponsored UV-B Critical Issues Workshop held at Cocos Beach, Florida, 24-26 February 1993. 


\subsection{Process Technology}




\title{
Advanced Catalysts and Catalytic Processes
}

\author{
T. D. Brewer (Materials Sciences)
}

The objective of this project is to develop solid acid catalysts that eventually can be used on an industrial scale to upgrade fossil fuels. Jointly conducted by PNL staff in collaboration with Washington State University, the project involves materials synthesis, characterization, and catalytic properties assessment. New synthetic methods are being devised to tailor the surfaces of certain metal oxides to yield highly acidic surface sites. These modified materials are then screened with respect to their activity and selectivity in catalyzing alkylation, isomerization, dehydration, and other reactions necessary to enhance the value of fossil feedstocks.

Prior scientific and technical efforts were directed toward synthesizing acid-treated aluminosilicates, titanates, and other metal oxides that were prepared by the glycine-nitrate process. These materials showed low catalytic activity for the isomerization of n-butane to isobutane due to the inability of the acid anions to react with the substrate surface.

Sulfated metal oxide catalysts containing titanium, zirconium, hafnium, iron, and tin recently have been synthesized through hydrolysis of the respective metal salts at elevated $\mathrm{pH}$. We have determined the optimum hydrolysis pH to be between 8 and 9 .

As a result of thermal and dynamic $x$-ray diffraction studies performed at Washington State University, the precipitated metal hydroxides appear to need a thermal treatment to remove water molecules adsorbed onto the surface. However, the thermal treatment should not be of sufficient temperature or time to remove hydroxyl groups. If the hydroxyl groups are removed or condensed, the metal oxide crystallizes and forms a stable phase which is resistant to sulfation. The resulting dehydroxylated materials, after sulfation, have proven to be ineffective $n$-butane isomerization catalysts. If the metal hydroxide is thermally treated at a temperature and time sufficient to remove water but not hydroxyl groups, the resulting material can be sulfated with $1 \mathrm{M}$ sulfuric acid, followed by a final heat treatment which condenses the sulfate group onto the crystallized metal oxide lattice. The resulting materials have shown n-butane isomerization conversions between $57 \%$ and $45 \%$ over a 15 -minute run at $200^{\circ} \mathrm{C}$ and a 0.2 -minute residence time. For comparison, a commercially supplied sulfated zirconia catalyst showed between $60 \%$ and $34 \%$ conversion using the same reaction conditions.

The catalytic activity results of sulfated zirconia show it to be the superior $n$-butane isomerization catalyst of the five sulfated metal oxides prepared. The other sulfated metal oxides were prepared using similar conditions as that of zirconia, and therefore they should be able to be further optimized. We are currently optimizing the synthesis of sulfated tin oxide, since tin is the only metal with a nuclear magnetic resonance active nuclei of the five metal oxides known to become superacidic upon sulfation. Nuclear magnetic resonance analysis will enhance our understanding of the bulk structure of these materials, the position of the sulfate group, and the location of protons which have been indicated by the presence of Bronsted acidity.

Various zirconia sources were tested and compared for $\mathrm{n}$-butane isomerization catalytic performance. The zirconium salts directly obtained from a commercial supplier proved to be inferior to those purchased from a generic chemical supplier. We believe that impurities present in the metal salts act as poisons in the final catalytic product.

Sulfated zirconia prepared by a different procedure also resulted in an active n-butane isomerization catalyst which deactivated quickly. The rapid deactivation is believed to be linked to the small particle size of this material.

Other Accomplishments

Follow-on funding from the DOE Office of Energy Research, Laboratory Technology Transfer Program, has been obtained as a result of this project. 


\title{
Advanced Coatings $^{(\mathrm{a})}$
}

\author{
G. L. Graff and B. C. Bunker (Materials Sciences)
}

New coating materials and techniques are important to industry not only to extend the useful life of components but also to allow the substitution of lighter-weight materials and to tailor the surface properties for specific applications. Program research has resulted in new, unique coatings techniques (i.e., biomimetic ceramic layers) and new coating materials such as inorganic polymers. The objective of this project was to demonstrate feasibility of these coatings techniques and materials on realistic parts.

Significant technical progress has been made in the areas of magnetic thin films and chemically resistant tin oxide coatings for potential use in the automotive industry. Synthesis of fully dense, oriented iron hydroxide films on polystyrene has been accomplished (Figure 1). The film is composed of microcrystalline, acicular akaganeite with crystallite orientation perpendicular to the substrate surface. These films have also been successfully converted to magnetic iron oxide at low temperatures $\left(<100^{\circ} \mathrm{C}\right)$. The high particle densities and perpendicular orientation of the magnetic phase could result in significant increases in storage density within the films. This is a major accomplishment toward development of novel magnetic films that can be directly deposited on polymeric substrates.

In an attempt to synthesize chemically and abrasion resistant coatings on plastics, conditions have been developed to deposit uniform, crystalline tin oxide thin films at $40^{\circ} \mathrm{C}$ from aqueous solutions. Transmission electron microscopy thin sections revealed that the tin oxide films are fully dense with $40 \AA$ crystallite size in the final film.

Though these initial findings were very promising, we later found that film deposition rates were extremely slow (days to weeks) and that film thickness was

(a) Project name will change in FY 1994 to "Synthesis of Advanced Coatings." limited to less than 2 microns. A detailed kinetics study of tin oxide growth was conducted to better understand the underlying mechanisms of film growth. Comparison of film thickness as a function of deposition time coupled with detailed transmission electron microscopy determined that there were two distinct stages of film growth. First, the ceramic coating rapidly intercalated within a thin (1 micron) surface gel layer of the polymer, followed by extremely slow growth of the ceramic phase on the outer surface of the plastic. By modifying solution conditions to favor film growth, coatings thickness increased by a factor of 3 and shortened deposition times to less than 24 hours. Currently, similar coatings are being attempted on acetal, polyvinyl chloride, and polycarbonate, which could have potential applications in the automotive and floor covering industries.

Investigations were initiated this year into the development of novel coatings on conductive substrate materials. Electrochemical methods were used to deposit sulfonic acid-containing polyoxophenylene films on metallic substrates. We have shown that these functionalized polymer layers will promote the formation of ceramic thin films from solution. By using photolithographically patterned electrodes, the technique can also be used to produce patterned ceramic films. This technique holds great promise for developing ceramic coatings on metals and unique ceramicpolymer composite materials.

Other Accomplishments

A number of important industrial interactions were initiated during FY 1993. A collaboration has been established with AC Rochester, a division of General Motors, to develop chemically resistant coatings for polymeric components used in automobiles. A small proposal was submitted through Dr. Arthur Yang of Armstrong World Industries on improved coatings for floor coverings. Efforts are currently under way to establish a cooperative relationship with Astro-Met Incorporated after demonstrating that the biomimetics process could successfully coat sponge nickel and beaded cobalt samples. Sample coatings were also supplied to Key Tronic and Chrysler Motor Company during FY 1993. 


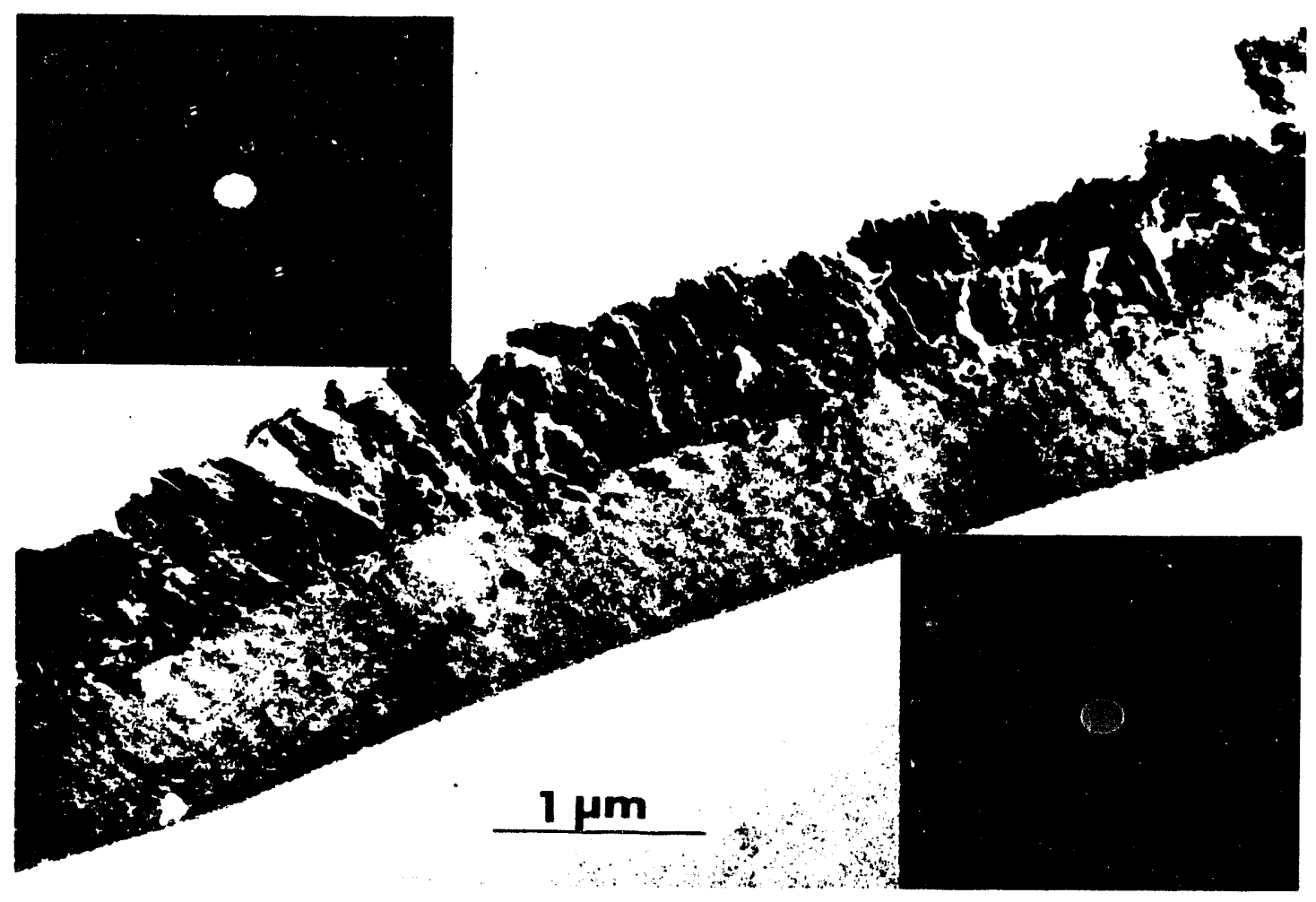

Figure 1. Iron Hydroxide Film on Sulfonated Polystyrene 


\title{
Fluid Dynamics Research
}

\author{
F. F. Erian (Analytical Sciences) and N. J. Lombardo (Applied Physics)
}

The objective of this project was to improve the ability to measure, understand, and predict the behavior of complex fluids. The complex fluids being addressed in this research project include classes of solid-liquid mixtures, non-Newtonian fluids, and colloidal suspensions, fluids that are often encountered in waste treatment and materials and chemical processing. The basic understanding of how these fluids behave in a processing environment is inadequate, leading to difficulties in process scale-up and inefficient processes. The initial focus is to perform experimental and theoretical investigations to better understand the effect of particles and rheology on the three-dimensional flow structure and behavior in process applications.

\section{Multiphase Flow of Solid-Liquid Mixtures}

\section{Homogeneous-Isotropic Turbulence}

(L. M. Liljegren)-During FY 1993, efforts were primarily focused on development of a unique test facility, which was designed for the collection of the desired data. All of its components have been acquired and partially assembled. The grid advancing mechanism has been tested and found to provide smooth and uniform motion ensuring that turbulence will be strictly due to the grid-induced fluid motion.

Velocity variances of both phases will be measured downstream of the grid using laser doppler velocimetry, which requires index-of-refraction matching of the solid particles and the liquid. Advances have been made in identifying index-of-refraction matched liquids which are also safe to use. Of equal importance are plans to use new particles with superior consistency of index of refraction and smaller variances in diameter between different batches.

Particle-Induced Turbulence (L. M. Liljegren)-In collaboratic : with Professor C. T. Crowe of Washington State University, additional data were gathered to improve our understanding of turbulence generation through mean slip in a multiphase solid/ liquid flow. Experiments were performed in a specially designed apparatus. This apparatus allows a given mass of solid particles to settle in a tank containing a liquid with an index-of-refraction that matches that of the solid particles. The path of the solid particles will be tracked optically and the characteristics of the particle-induced turbulence field will be measured using laser doppler velocimetry techniques. In FY 1993, efforts were focused on designing and building the test facility, and on finalizing the planned experimental program. Measurements are planned to start in the second quarter of FY 1994.

Boundary Interaction Experiments (J. D. Hudson, J. R. Phillips)-An important but often ignored aspect of multiphase flow is the description of the particle phase behavior near a solid boundary. In FY 1993, an extensive literature review was conducted to clarify key technical issues in solid-liquid slurry flows. The review led to the initiation of plans to conduct a set of fundamental experiments which consider the boundary interactions and turbulence in slurry pipe flows. The first of these experiments will focus on obtaining velocity information of the dispersed phase very near a pipe wall. The data will be used to validate engineering codes and provide an experimental reference to current analytical models.

A new experimental facility has been designed for these experiments. This facility will be constructed to allow highly detailed experimental measurements using optical techniques such as laser doppler velocimetry and particle image velocimetry with refractive-index-matched slurries. Procurement and fabrication of many of the key components have already begun. These include a four-stage progressing cavity pump capable of handling large particulates at high-volume fraction, a $10-\mathrm{kW}$ chiller and a state-ofthe-art motion control system for the optical equipment. The facility will be housed in the Materials Research Center building with anticipated construction and assembly to start late 1993.

The refractive-index-matched slurry used in the initial experiments will consist of solid acrylic particles in a liquid mixture containing a surfactant, a lubricant 
additive, and a halogenated hydrocarbon. This slurry provides an index match between the fluid and particulate with a density ratio that is tunable within a small range, affording experiments which require neutral buoyancy. In addition, this mixture is essentially nonflammable and has insignificant environmental impact when compared $w$ th other alternatives. Small quantities of the mixture save been prepared and transparency has been verified.

\section{Non-Newtonian Fluid Dynamies}

\section{Multiphase Non-Newtonian Mixing}

(A. Shekarriz)-The flow behavior of a submerged pseudoplastic jet was investigated using particle image velocimetry. The purpose was to determine the impact on mixing effectiveness of non-Newtonian jets. Due to its shear-thinn'ng characteristics, a pseudoplastic jet's forward velocity was found to drop suddenly (to near zero) at some distance downstream of the jet exit. This distance is a function of both nozzle exit velocity and fluid rheology-it may extend only a few jet diameters downstream of the jet exit. It was also found that the mixing rate and effectiveness in this case is substantially lower than for a conventional Newtonian jet at the same Reynolds number. Since some of the tank wastes at Hanford have been characterized rheologically as being pseudoplastic, these results can have a significant impact on tank waste mobilization, and on achieving the desired level of uniformity. The results of this study have been submitted for publication to the ASME Transaction Journal of Fluids Engineering, and will also be presented at the 1994 ASME Fluids Engineering Division meeting. An LDRD seminar entitled, "Application of Particle Image Velocimetry in Studying the Dynamics of a Non-Newtonian Mixing Jet," which contained a summary of this work was presented on July 1, 1993.

In a separate study, the creeping flow of a yield pseudoplastic fluid around a sphere was studied using particle image velocimetry. The flow adjacent to the sphere's front and rear stagnation points was found to be asymmetric. This is unlike observations of Newtonian fluid flow around a sphere at low Reynolds numbers. Moreover, a stationary toroidal or ring vortex appeared along the side of the sphere which had not been observed previously. Falling-ball viscometry has recently been proposed to obtain in situ characterization of tank 241-SY-101 waste. The current findings will be useful in assessing the accuracy of this approach. The results of this study will be presented at the Fluid Dynamics Division meeting of the Arierican Physical Society in November 1993.

\section{Advanced Instrumentation for Fluid Dynamics Research}

\section{Developments in Optical and Ultrasonic Imaging}

(A. Shekarriz)-Development of a state-of-the-art particle image velocimetry system, which was designed in FY 1992, was successfully completed. Particle image velocimetry was used for planar instantaneous measurement of the velocity field within a turbulent jet. The system was tested and shown to be capable of accurately resolving turbulence scales as small as $1 \mathrm{~mm}$. This measurement technique can be used to study complex fluid flow geometries such as pulsating jets and opposing coaxial jets. Both are of interest in mixing enhancement.

An existing ultrasonic imager, known as RTUIS (Real-time Ultrasonic Imaging System) was implemented to measure the velocities within an optically opaque mixture. It was feasible to measure instantaneous velocities in mixtures such as liquid paper pulp, polymeric fluids, and pharmaceuticals. The results of this study have been submitted for presentation at the 1994 ASME Fluids Engineering Division meeting, and for publication in the ASME Transactions Journal of Fluids Engineering.

An existing laser doppler velocimetry system was upgraded for use as an advanced research tool and as a process monitoring system with an enhanced signal processing capability. The system will permit measurement of local instantaneous velocities at sampling rates as high as $4000 \mathrm{~Hz}$. Useful information about turbulence characteristics such as time-averaged and RMS velocities, auto-correlation and cross-correlation of velocities can also be determined.

\section{Publications}

Five journal articles; two accepted and three being reviewed, and numerous conference papers were published/presented by members of the Fluid Dynamics Laboratory team in a broad range of research fields.

\section{Presentations}

A seminar which described the capabilities of this system, entitled, "Application of Particle Image Velocimetry ... Unsteady and Turbulent Flows," was presented at the Department of Mechanical Engineering, Washington State University, in April 1993. 
The Invited Locturer series continued its first year success with over ten nationally recognized researchers visiting the Laboratory. They presented seminars, discussed research activities and explored opportunities for potential cooperation on research problems of mutual interest.

The fluid dynamics laboratory organized a national workshop on particulate flow which included over 50 participants from academia, industry, and government. Professor Harri K. Kytömaa of Massachusetts Institute of Technology, as a result of attending the workshop, decided to spend part of his forthcoming sabbatical leave at PNL. He will be working on fluid dynamics laboratory projects with various team members.

A team member was invited to participate in a DOE/NSF conference on particulate flows.

\section{Other Accomplishments}

Continuing LDRD funding permitted the completion of three new flow imaging systems for the characterization of multiphase particulate flows. The first is the currently fully operational particle image velocimetry system, the second is an upgraded two-component laser doppler velocimetry system, and the third is the RTUIS system.

Dr. Fadel F. Erian, with academic and industrial experience (12 years at Clarkson University and 15 years at Shell Development Co.), and Dr. John Hudson (University of Illinois) were hired.

Five M.S.-level NORCUS students from three universities participated in our research programs. A Ph.D. candidate at Washington State University has a National Science Foundation fellowship and is collaborating with us in the Particle-Induced Turbulence project. Two members of the fluid dynamics laboratory team were appointed as advisors to Ph.D. candidates, and as Adjunct Professors at Washington State University.

Industrial contacts and potential cooperations are being explored with the paper and pulp industry (Weyerhauser, James River) and the petrochemical industry (M. W. Kellogg). 


\title{
In Situ Monitoring of Ultrafine Particle- to-Particle Material Interactions
}

\author{
R. H. Jones and J. Liu (Materials Sciences)
}

This research is directed toward the science of ultrafine (nanometer) particle and particle-toparticle interactions related to synthesis and consolidation. The objective was to understand the characteristic properties of these nanometersize particles, the interactions that occur between the particles, the role these properties have during consolidation processes and a comparison with consolidation of larger, micrometer particles. The approach includes the property characterization of nanometer particles, the determination of particle interaction, and the in situ monitoring of the particles during consolidation. The research provided an introduction into a new and emerging science that complements science and engineering already being conducted.

Ultrafine or nanometer-size particles exhibit unique chemical, structural, and thermophysical properties, which result from their small size (1 to $100 \mathrm{~nm})$ and large interface areas. In particular, metals and ceramics consolidated from non-agglomerated, nanometer particles can also exhibit unusual processing characteristics that vary significantly from those consolidated from larger particles. Structures with grain sizes of 5,10 , and $100 \mathrm{~nm}$ have approximately 50,30 , and 2 vol\% respectively, of the atoms associated with the grain boundary interfaces. Structures of this nature can result in high atom diffusivities and enhanced solute solubilities. Reactivities are high. The ionic and electronic transport thror th grain interfaces generally differ from bulk transport. Materials, especially ceramics, may exhibit high ductility, even at low temperatures. For example, 5 to $10 \mathrm{~nm}$ silicon nitride particles can be consolidated at cryogenic to near theoretical density at moderate pressures. Sintering temperatures are dramatically reduced.

The properties of these nanometer-size particles and the materials formed from these nanometer particles appear to be strongly influenced by particle size, morphology, particle-to-particle interactions, and structure. Nanometer particles are easily attracted to and react with one another. The nature of these attractions and bonding are not understood. The role of the characteristic interactions of ultrafine or nanometer metal and nonmetallic particles in the consolidation or other processes are also not understood. Understanding these relationships should enhance the ability to control, synthesize, process, fabricate and design metal and ceramic materials with selected mechanical, thermal, electrical, chemical, and electrochemical properties.

Recently, new methods for the synthesis of nanometer metal and nonmetallic particles in large quantities have been developed within PNL and in other research institutes. Research into the characteristics of these particles is very limited and many advanced characterization methods have not been used to determine the properties of the nanometer particles. Thus, research to study the role of the unique properties of nanometer particles on processes, such as consolidation is also limited.

The objective of this research was to understand and model the role of nanometer particle properties and particle-to-particle interactions on consolidation processes. The research included the characterization of nanometer particle properties, the interaction between ultrafine particles, and the comparison of these properties with micrometer-sized particles. The initial research emphasized nonmetallic nanometer particles.

Initial review of literature and previous studies revealed that the difference between large particles and nanometer-size particles may be the relative importance of the interaction forces between particles. The interaction and behavior of colloidal systems of large particles can be described by traditional Derjaguin-Landau-Verwey-Overbeek theory. However, for ultrafine particles, the near surface structural forces may dominate the properties.

These structural forces may include hydration forces, short-range electrostatic interactions, forces due to the 
adsorption of impurities and foreign atomic or molecular species, and forces due to complex hydroxide formation. These forces can be potentially harnessed to control the rheological and consolidation behavior of such systems.

In order to understand the unique interactions between nanometer-size particles in colloidal suspensions, especially the effect of the near surface forces, the aggregation behavior of ultrafine colloidal silica and zirconia was studied under different conditions. A dedicated laser light scattering system was established to study the colloidal suspensions, and Doppler shift light scattering was used to monitor the kinetics. The aggregation of ultrafine colloidal particles is of fundamental importance in materials processing and other applications. The scaling behavior of the aggregation kinetics and the aggregate structures are directly related to the particle interactions. One of the purposes of this research is to study the effect of the solution and surface chemistry on the bonding and structural formation. We studied the aggregate structures of colloidal silica and zirconia in different electrolyte solutions at different concentrations by static light scattering and contrasted the results with those reported in literature from fast and slow growth mechanisms. We also compared the results with those involving reversible aggregation and restructuring processes. The electrolytes are chosen on the basis of the number of charges, hydration radii, and ion exchange ability, all of which play important roles in the short-range structural forces and the aggregation rates.

We found that a higher electrolyte concentration and a faster growth kinetics would give a higher fractal dimension. The fractal dimension $d_{\mathrm{f}}$ varies from 2.0 to 2.4 for silica, and 2.2 to 3.8 for zirconia, depending upon the specific electrolyte and electrolyte concentration. In Figure 1, we present the results from the silica in $\mathrm{Mg}\left(\mathrm{NO}_{3}\right)_{2}$ solution of different concentrations. At low electrolyte concentrations ( 0.8 molar) the aggregation took place over a period of several weeks and the fractal dimension is about

2.1. This is in agreement with the results reported in reaction limited aggregation. However, at higher electrolyte concentrations the fractal dimension begins to increase with salt concentration. The fractal dimension at 2 molar $\mathrm{Mg}\left(\mathrm{NO}_{3}\right)_{2}$ is 2.29 and at 4 molar becomes 2.37. These numbers are higher than those reported in the literature, and it is surprising that higher fractal dimensions are observed with higher salt concentration and faster kinetics. One would expect the opposite effects: rapid aggregation and high electrolyte concentration would result in a low fractal dimension because the diffusion limited process does not allow the particles to settle to a low energy state and would give a more ramified structure.

The aggregate structures of colloidal zirconia in various electrolyte solutions are summarized in Figure 2. In general, the fractal dimension of zirconia ranges from 2.3 to 3.8 , and the results are also related to the rate of aggregation: the faster the aggregation, the higher the fractal dimension. Only in the case of zirconia is the effect more pronounced, which may be related to the smaller particle size of zirconia. The slower aggregation with $\mathrm{Al}_{2}\left(\mathrm{SO}_{4}\right)_{3}$, which took place over 10 days as determined by visual inspection, gives a fractal dimension of 2.3 , closer to the result from reaction limited aggregation. However, the faster aggregation with $2 \mathrm{M} \mathrm{Mg}\left(\mathrm{NO}_{3}\right)_{2}$, which took place in several hours, gives a much higher fractal dimension of 3.8. Such aggregates are no longer mass fractals, but can be treated as surface fractals.

These observations are different from previous investigations, where the aggregation occurred by the fast and slow aggregation models, and cannot be explained by other models involving restructuring and bond breaking. In the fast growth regime, there is no energetic barrier between particles and the growth rate is limited by the diffusion kinetics of particles and clusters (diffusion limited clustering aggregation). The fractal dimension is about 1.8 and the growth kinetics has a power law relationship. In the slow growth regime, the aggregation is modified by a finite sticking probability due to the existence of an energetic barrier between particles and is, therefore, called reaction limited aggregation kinetics. The fractal dimension is 2.1 and the growth kinetics has an exponential relationship. We attribute the high fractal dimensions associated with high electrolyte concentrations and fast kinetics to osmosis driven structural shrinkage due to the screening of the charges presented near the particle surface by the background electrolyte. A similar phenomenon has been well documented in polymeric gels. Such structural reorganization occurred in our experiments because the bonds between the particles were modified by the ionic species near the particles.

Parallel to the study of colloidal particles, computational tools for investigating fundamental aspects of particle-particle interactions at the atomic scale were 
developed. Current studies are limited to a simple system of crystalline nanoparticle nickel. The interaction of two identical nanoparticles $2.5 \mathrm{~nm}$ in diameter (containing 767 atoms each) was characterized using molecular dynamics with embedded atom method interatomic potent :.ls. Particle interactions were simulated at temperatures up to $900 \mathrm{~K}$ for times up to 183 ps. Analyses of the molecular dynamics simulations were performed to investigate surface energies, particle force-distance relations, and neck growth kinetics at the interface, including surface and bulk self-diffusion of individual atoms. The preliminary

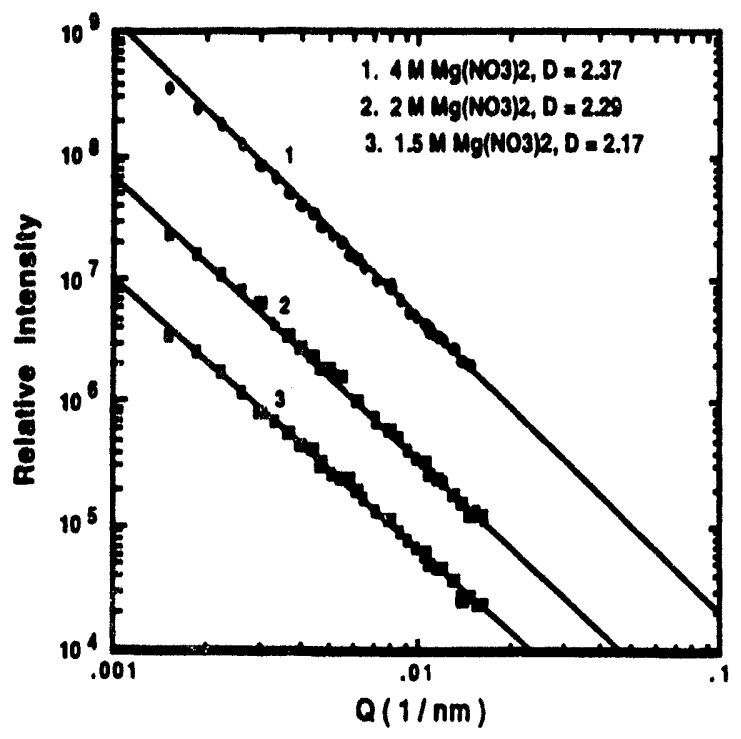

Figure 1. $\mathrm{SiO}_{2}$ Aggregates in $\mathrm{Mg}\left(\mathrm{NO}_{3}\right)^{2}$ results will be compared to those obtained from other theoretical methods, and will be evaluated in terms of grain boundary stabilities and densification mechanisms.

\section{Publication}

Liu, J., R. E. Williford, H. Heinisch, J. Virden, G. Graff, and R. H. Jones. "Unusual Colloidal Aggregates of Ultrafine Ceramic Particles." Pacific Northwest Laboratory, Richland, Washington.

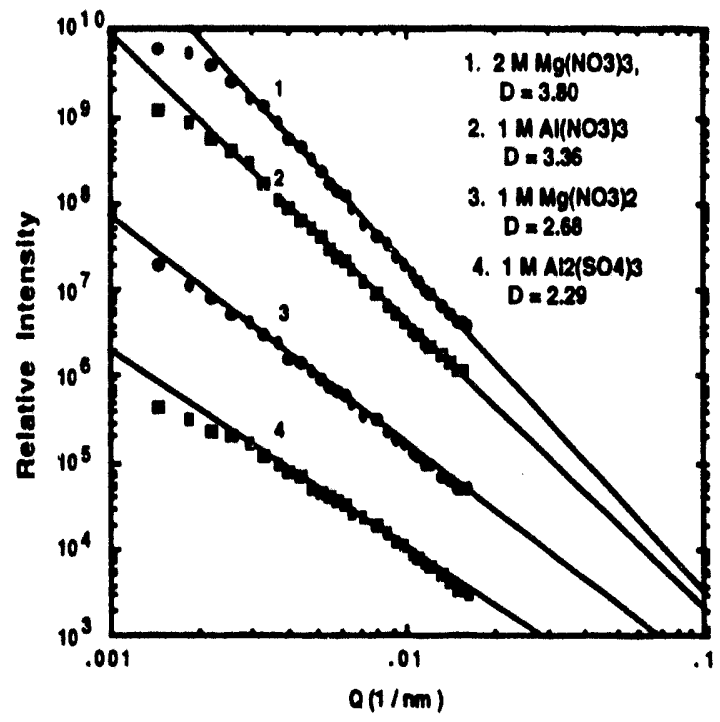

Figure 2. $\mathrm{ZrO}_{2}$ Aggregates in Different Electrolytes 


\section{Materials Evaluation}

R. A. Pappas, S. R. Doctor, and B. P. Hildebrand (Automation and Measurement Sciences)

The United States infrastructure is in rapid decline due to neglect and lack of understanding of behavior of materials in the harsh environments they are subjected to. The primitive state of nondestructive material characterization methods prevents timely detection and measurement of the extent of material degradation, which may subsequently lead to catastrophic failure. The intent of this research was to initiate a systematic scientific investigation of the interaction of a variety of probing modalities with the material in order to extract material parameters useful in predicting failure and estimating remaining life of the structure under test.

\section{Birefringence Measurement Systems}

Two reliable and reproducible measurement systems were developed for the purpose of acquiring acoustic birefringence data. One system was designed around the use of conventional ceramic transducers. The other system employed novel ultrasonic transduction methods. In this system, a neodymium glass laser was used as a broadband ultrasound generator. A broadband receiver was designed and fabricated using piezoelectric polymer film. An electromagnetic acoustic transducer receiver, narrowband in response, was also used to detect the laser-generated ultrasound.

\section{Preliminary Measurements}

Acoustic birefringence (shear wave velocity directional dependence) has been demonstrated as a sensitive measure of material property anisotrophy. Preliminary data collected in FY 1993 suggests a frequency dependent birefringence response to a microstructure feature, carbide precipitation, which directly affects engineering performance. Birefringence measurements were made at $1,2.5$, and 6.5 MHz using stainless steel samples. The samples had been subjected to heat treatments resulting in a variation in carbide precipitation.

The same samples were investigated with the lasergenerated ultrasound system. Preliminary results with this system suggest an alternative, reliable measure of microstructure. This method promises to be quicker since all frequency information is acquired in a single measurement.

Both birefringence measurement techniques yielded results in FY 1993 which were suitable for publication. The manuscripts will be completed and submitted in early FY 1994. 


\title{
Materials Synthesis
}

\author{
G. L. Graff and B. C. Bunker (Materials Sciences)
}

The Materials Synthesis Project is aimed at synthesizing and modeling the behavior of inorganic ion exchange materials of potential use in separating cesium and other hazardous radionuclides from the mixed wastes stored in Hanford tanks. The objective of the project is to understand the properties of exchange materials to the point where existing materials can be implemented in actual tank separation processes and to identify gaps in the existing ion exchange technologies.

In FY 1993, four major activities were pursued in synthesizing and understanding inorganic ion exchangers 1) large-scale synthesis of inorganic ion exchangers via Rapid Thermal Decomposition of precursors in Solution (RTDS), 2) investigations of the consolidation of inorganic exchangers into pellets for exchange column operation, 3) theoretical modeling of ion transport in zeolites, and 4) studies of ion exchange mechanisms in pillared zirconium phosphates. Below, progress on each of the above tasks is described.

Rapid Thermal Decomposition of Precursors in Solution Synthesis of Inorganic Ion Exchangers

Most inorganic ion exchangers having high exchange capacities consist of high surface area nanometer-size crystallites. Several classes of such materials, including silicotitanates, zeolites, and zirconium phosphates are often prepared using hydrothermal techniques. Workers at PNL have developed a continuous hydrothermal process which is capable of rapid production of kilogram quantities of nanoparticle materials. The technique, call Rapid Thermal Decomposition of precursors in Solution, involves heating aqueous solutions containing soluble metal salts or other precursors to temperatures in excess of $200^{\circ} \mathrm{C}$ to induce nucleation of nanocrystallites. The solutions are then quenched by spraying into aqueous solutions held at room temperature. This year, the existing laboratory-scale process was scaled to near pilot-plant scale. Using the new pilot-scale facilities, a variety of inorganic powders were prepared to demonstrate the capabilities of the system including oxides of iron, zirconium, titanium, zinc, nickel, and copper. Mixed systems including nickel ferrite were also prepared. The system should now be ready for attempting the synthesis of actual inorganic exchange materials in the coming fiscal year. In addition, extensive large-scale hydrothermal equipment should be ready for deployment.

\section{Pelletization of Inorganic Exchangers}

Although several inorganic exchangers exhibit high distribution coefficients for extracting cesium from tank wastes, the best materials are currently unsuitable for actual deployment in an ion exchange process. This is because the exchangers are nanoparticle materials. Liquid wastes cannot be forced between the small pores present in a packed column of nanoparticles. In addition, the nanoparticles cannot be completely contained. Escaping particles lead to undesired transport of trapped radionuclides. Therefore, several methods are under investigation to try to consolidate the particles into an engineered form such as a pellet which can be used in an ion exchange column. This fiscal year, pellet pressing techniques were evaluated. Most pressing operations involve use of organic binders to improve bonding between the particles. Unfortunately, commercial organic binders which were evaluated cannot survive the extremely basic solution $\mathrm{pH}$ values seen in tank wastes and are also susceptible to radiation damage. Therefore, initial efforts at pelletization investigated binderless pressing. Results on titania particles show that surprisingly hard pellets with excellent structural integrity can be produced using binderless pressing. Unfortunately, the pressing operation reduces the ion exchange properties of the base material. In addition, under column operation conditions, swelling due to aqueous intrusion degrades the structural integrity of the pellets. Therefore, alternates to binderless pressing will be investigated if the nanoparticle consolidation project continues.

\section{Modeling of Ion Transport in Zzolites}

Inorganic ion exchangers with the highest selectivity for cesium are thought to obtain their selectivity through steric effects. The best exchangers are those 
which have pores or layer spacings which can admit cesium ions but which are too small to admit hydrated sodium ions. To gain an understanding of the role of steric effects in inorganic ion exchangers, molecular modeling studies were performed on zeolite materials. In the models, zeolites were selected with different ring openings. Cations having different ionic radii, such as sodium, potassium, and cesium were selected as model cations. Theoretical calculations were performed to obtain diffusion coefficients for different ratios of cation diameter to ring opening. The results show that if the ionic diameter is smaller than the opening diameter, ionic diffusion in zeolites can be rapid, while if the ring opening is smaller than the cation, ionic diffusion is exceedingly slow. The results are in agreement with experimental results which indicate that steric effects are indeed critical to the selectivity and exchange kinetics for inorganic exchangers.

\section{Pillared Zirconium Phosphates}

Professor Abraham Clearfield at Texas A\&M University has developed a family of pillared zirconium phosphate materials which PNL and Allied Signal Corp. are evaluating as candidates for removal of cesium from tank wastes. Zirconium phosphates are layered clay-like materials having anionic phosphate groups between the layers. By using organic phosphonate groups (organics with phosphate groups on each end), Professor Clearfield has been able to systematically control the spacing between the zirconium phosphate layers. This year, through a contract with Professor Clearfield, we were able to obtain a series of zirconium phosphate samples to test if steric effects control their cesium selectivity. Samples were obtained in which both the separation distance between the zirconium phosphate layers and the spacing between the pillars were systematically varied. Ion exchange experiments performed at PNL show that the cesium selectivity is indeed sensitive to pillaring. However, it appears that the spacing between pillars has a greater impact than the spacing between the layers. The study also revealed that previous values reported for cesium selectivity of the zirconium phosphates were in error. It appears that the zirconium phosphates are chemically unstable in basic tank wastes and are inferior to other inorganic exchangers under consideration such as silicotitanates.

\section{Other Accomplishments}

The pilot-scale Rapid Thermal Decomposition of precursors in Solution system was awarded an R\&D 100 award from R\&D Magazine as an outstanding research development in 1993. 


\title{
Multicenter Hazardous and Radioactive Waste Treatment and Remediation
}

\author{
W. A. Ross (Waste Treatment Technology)
}

This project addresses the need for hazardous and radioactive waste treatment and remediation on DOE sites with special recognition of Hanford needs and priorities. Research areas in the past 3 years included biological treatment of hazardous organics, removal of radionuclides from groundwaters and soils, treatment and handling of wastes both in situ and ex situ by innovative processes and methods, low-temperature treatment of offgas, development on high-waste-loaded glasses, destruction of organics, collection and evaluation of disposal site characterization data, and monitoring methods for in situ treatments.

Destruction of Organic Contaminants in Aqueous Waste Streams (J. W. Virden, G. Pillay, D. M. Camaioni, G. M. Mong, L. Laverman, R. R. Shah)

It was previously noted that corona discharges above the surface of water can lead to destruction of organic compounds dissolved in water. During FY 1993, three related activities were conducted 1) experimental studies were conducted to elucidate mechanisms of contaminant destruction and scale-up parameters for corona discharge apparatus, 2) scale-up experiments were conducted with multiple electrodes and elevated voltages and currents, and 3 ) investigations were conducted into the design of a wet acid liquid scrubber design for the corona off-gas system. The following are the results of these activities.

Experimental studies showed that the yield of oxidants depends on the oxygen content of the gas above the liquid, ozone and hydrogen peroxide are produced during the corona events, and that the radicals do react with methanol as a representative organic. It was also noted that off-gas from a low-temperature plasma reactor exhibits oxidizing powers in liquid-gas contractors.

The multiple electrode tests demonstrated that the onset of corona was generally observed at about $8 \mathrm{kV}$ and that the corona became much brighter as voltage and current increased. It was also noted that configurations for multiple electrodes need to be carefully designed to avoid undesired corona. Further, it was noted that reaction rates increase with electrical current, but not necessarily at a linear rate.

The design of an initial wet acid liquid scrubber was completed and equipment prepared for further testing.

\section{Glasses for Idaho Chemical Process Plant Calcine} (R. D. Peters, K. F. Whittington, R. R. Adee, D. Bonner, J. D. Vienna)

This work investigated the potential of developing high-waste-loaded glasses for the Idaho Chemical Process Plant (ICPP) high-level-waste calcine. Recent experience with glasses and a careful review of the glass composition data base information indicated that a much higher than the previous $33 \mathrm{wt} \%$ loading of calcine in a glass waste could be obtained. Glass formulations, using surrogate materials, were developed with waste loading as high as $65 \mathrm{wt} \%$. Even with the high waste loading, glass melting and processing temperatures remained low at about $1150^{\circ} \mathrm{C}$. Representative glasses were leach tested and found to have similar durability to other high-level-waste glasses. The results suggest that vitrification may indeed offer a cost-effective approach to treatment of the Idaho Chemical Process Plant calcines.

\section{High Oxygen Overpotential Electrodes (J. E. Surma)}

The objective of this program was to further develop and test electrode materials for application as high overpotential electrodes. Electrochemical organic destruction involves the destruction of organic materials in an electrochemical cell and has wide potential applications in treatment of mixed wastes and tank wastes.

The majority of this year's effort involved the fabrication of the high overpotential electrodes. Four different electrodes were prepared and tested. The 
results of this effort indicate the high overpotential electrodes can be used to effectively destroy organic species in Hanford tank wastes.

This LDRD effort has resulted in a new program to evaluate the high overpotential electrodes in an electrochemical organic destruction process for Hanford tank waste.

\section{Holography for Buried Wastes (H. D. Collins, R. P. Gribble)}

This work evaluated the potential of using ultrawide band radar holography to detect objects buried in the soil. Radar impulse holography can simply be described as a multifrequency detection and imaging technique where the target's broadband time wave from signals are recorded over a defined aperture; decomposed into their discrete frequency components as single frequency holograms, and reconstructed into a composite image by computers. Previous work for the military had indicated possibilities for this technology for investigation of buried waste sites. This activity tested the ability to detect objects buried at various depths in the soil. The tests were successful and resulted in a contract with the Buried Waste Integrated Program for further development of the technology.

Hybrid-Treatment Process (W. A. Ross, J. S. Tixier)

The Hybrid-Treatment Process has been conceived as a process that will provide robust low-cost treatment of stored and retrieved low-level wastes.

The process as conceived accepts and treats the wastes in their original drums or boxes. Following limited characterization, the wastes will be directly loaded into a large (approximately 35 -foot-diameter) processing vessel without further sorting or size reduction, except to remove high transuraniccontaining wastes and high hazard materials such as explosives or compressed air cylinders. The processing vessel is assembled in the disposal area below grade. No backfill on the outside of the vessel is emplaced before processing to allow vessel wall cooling during the high-temperature processing period. After loading, the vessel is heated slowly from internal electrical heating to vaporize water and other volatiles. Volatilized materials are treated in mobile trailers in an off-gas treatment system that provides organic vapor destruction by either flame burning or plasma destruction technologies. After the volatiles are removed from the drums and boxes by low temperature heating, the temperature is raised to allow complete destruction of organic materials and eventually melting of the wastes and their homogenization by the convective currents in the molten waste glass. This activity provides a proof-ofprinciple test for the hybrid-treatment process. The test was conducted at a scale of about $1 / 30$. The test demonstrated many of the principles of the HybridTreatment Process concept, including that it was possible to slowly heat the waste drums using a graphite and soil mixture and that contained drums would not rupture with sufficient energy to upset the off-gas treatment system. Additional follow-up testing is now needed.

\section{Porous Optical Fiber Sensor Development (K. A. Stahl)}

The objective of the project was to investigate the applicability of porous optical fibers to DOE environmental restoration and waste management chemical sensing needs and test at least one sensor concept. The measurement of hydrogen was selected as the test analyte. It was expected that such a sensor would offer small size for minimally intrusive sensing, remote and explosion proof operation, as well as the other usual benefits of optical fiber sensor systems.

The physical basis for the sensor is the adsorption of hydrogen by the palladium to form $\mathrm{PdH}_{\mathrm{x}}$ (x depends on partial pressure of hydrogen). The $\mathrm{PdH}_{\mathrm{x}}$ has a larger lattice constant than Pd, so it is thought that the formation of the hydrides throughout the pores in the fiber will stress the fiber and increase the optical losses in the fiber through increased scattering and possibly increased absorption. The relative spectral transmission of the Pd-loaded fiber was measured. A test system consisting of the diode laser source, focusing optics, optical chopper, lock-in amplifier, fiber holder/gas cell, and AlGaAs photodiode/pre-amp was assembled. To test the sensor concept, a Pdloaded fiber was held in the gas cell so that the loaded portion was completely inside the cell. While monitoring the transmitted light signal level, hydrogen was introduced to the gas cell. The results of the experiment were negative-no meaningful change in optical signal level was detected. 
In Situ Fungal Barriers (M. J. Truex, P. J. Prado, F. J. Brockman, G. Globus)

The original goal of this activity was to determine a means to install a fungal barrier in the vadose zone to reduce or prevent waste migration. The initial steps in the process, injecting the fungal materials into a soil and promoting its growth, was the emphasis of the work this year. During the year, technology for inoculating soil in situ with P. chrysosportium was demonstrated. Alternative uses of the technology were identified. One application of this procedure includes treatment of unsaturated soil that is contaminated with relatively immobile and non-volatile contaminants that can be degraded by specific fungal strains and are amenable to the injection solution and subsequent air injection. Further work on the techniques and methods is still needed.

\section{Separation of Tritiated Water from Water (D. A. Nelson, G. A. Jensen, J. B. Duncan)}

The objective of the work was to develop and evaluate a polymer membrane for use with the concept of pervaporation for separation of HTO from $\mathrm{H}_{2} \mathrm{O}$. This was to be achieved by 1 ) identifying suitable polymer formulations that can separate tritiated water from ordinary water, 2) producing polymer membranes for the needed separation, and 3) testing and evaluating the membrane to determine the separation efficiency for tritiated water.

To accomplish the separation, poly(dipenoxyphosphazine) was chosen for the membrane polymer due to its high degree of radiation stability. This polymer is also chemically and thermally stable. Early work established that approximately $10 \%$ carboxylation of the phenoxy-groups provided a useful material. Initial experiments were completed to cast and test the membrane and complete preliminary feasibility studies for separation of HTO from well water obtained on the Hanford Site. The well that was chosen produces water that did not contain hazardous waste, yet did contain tritium at concentrations of approximately $10,800 \mathrm{Ci} / \mathrm{L}$, i.e., HTO levels below the drinking water standards of $20,000 \mathrm{pCi} / \mathrm{L}$. Working at that level enabled laboratory work without going into a radiation zone.

The results have been quite encouraging. Reductions of $10 \%$ to $20 \%$ of HTO were first seen in early proofof-principle experiments. As the proof-of-principle experiments progressed and selected membrane formulations were tested, reductions of up to $74 \%$ in the HTO concentration of the well water were measured. The flux of HTO across the membrane has been without phase change and therefore, less energy intensive than other processes (cryo-distillation, electrolysis/catalysis) which need higher concentrations of HTO to be cost-effective.

\section{Development of Plant Rhizosphere-Based Containment and Biorestoration Technology (M. Kingsley, B. Metting, J. K. Fredrickson, R. J. Seidler)}

The objective of this project was to collect basic information for planning and analysis leading to fundamental scientific investigation of the use of natural and genetically modified plants and rhizosphere organisms for reniediation and restoration of contaminated soils.

Results from this activity include

- a literature data base on rhizosphere degradation/biorestoration was assembled

- additional applications were identified to employ plants and associated rhizosphere microbes (natural and genetically manipulated) for the remediation of uranium and nitroaromaticcontaminated soils

- presentations were made at two international meetings on results of preliminary studies employing plants and rhizosphere bacteria to degrade a herbicide

- rhizosphere-competent microbial strains, with the potential to degrade trichloroethylene were constructed via plasmid transfers.

\section{Non-Leaking Fluids for Single-Shell Tanks (E. V. Morrey)}

Literature and laboratory studies were performed to determine feasibility of using diluted colloidal silica as a non-leaking retrieval fluid for single-shell tank waste. The diluted colloidal silica when mixed with the highly alkaline waste produces a thixotropic gel. Laboratory studies included measurement of rheological and soil permeability properties of various waste/colloidal silica mixtures as a function of time. The results indicated that a wide range of waste/ colloidal silica mixtures produce the desired properties 
(i.e., strong enough to prevent leaks when not under shear and thixotropic enough to be mobilized and retrieved). Preliminary measurements showed that the gels may be sufficiently reversed by $\mathrm{pH}$ adjustment to meet transport and vitrification plant slurry specifications. Soil permeability measurements of the waste/colloidal silica mixtures were $\geq 4$ orders of magnitude less than mixtures of waste and water, which indicates that if the gel mixture did reach the soil column, it will be relatively immobile. 


\title{
Organic Conversion
}

\author{
T. M. Bergsman (Waste Treatment Technology)
}

This project was focused on understanding and developing low temperature plasma processes. A low temperature plasma is produced when a strong electric field is established in a gas, causing the gas to partially ionize, forming $a$ plasma. This mechanism can be used to create reactive conditions to destroy contaminants. In addition, the technology has a potentially much broader application in the area of synthesis (e.g., oxygenates for fuels) and electrocatalyzed processing for unique industrial processes. The overall goal was to link fundamental research, process modeling, technology development, and applied research to significantly enhance the state of the art in this promising area.

\section{Flowing Afterglow Apparatus}

A state-of-the-art diagnostic apparatus was designed and constructed ( $90 \%$ complete). The device, the flowing afterglow apparatus, will be able to identify neutral and ionic products of the corona discharge. The effluent from a corona reactor will be sampled and transported to a detector in a rapidly flowing bath of inert gas at low pressure. Ionization of neutral species will be carried out by electron impact, penning ionization or charge exchange with specific ionic precursors. The ions are sampled into a doubly differentially pumped quadrupole mass spectrometer for detection and identification. The detection of the reactive species produced in the corona reactor will assist in understanding the molecular reaction mechanisms associated with corona processes to develop enhanced reactor designs.

\section{Electric Field Modeling}

An electric field model was developed for the ac voltage-current relations in a cylindrical geometry packed bed corona reactor. A model specialized to the packed bed corona reactor was presented giving quantitative predictions of dependence of the voltage threshold for corona in the packed bed geometry and electrical properties, including dielectric constant of bed material and thickness and dielectric constant of the cylindrical shell. This conceptual model can provides insights to scale-up and alternate reactor configurations.

\section{Prototype Advances}

Various catalytic materials were tested to increase perchloroethylene and carbon tetrachloride destruction efficiency. A greater than fourfold increase in reactor capacity was achieved using these catalysts. Significant further increases in reactor capacity are expected based on catalyst configuration and material. In addition, an acid gas removal system was designed and is currently being tested. The system will allow the removal of acid gases (primarily $\mathbf{H C l}$ ), as well as a mechanism to enhance destruction efficiency by enabling byproducts to be destroyed in the liquid phase.

The enhanced system was used to test carbon tetrachloride, a contaminant currently of concern at an Expedited Response Action at Hanford. Carbon tetrachloride destruction efficiency was $97.1 \%$ at 5.3 seconds residence time and $25 \%$ inlet $\mathrm{RH}$, while at $80 \%$ inlet $\mathrm{RH}$, the destruction efficiency was 91.6\% (expected field conditions). Destruction testing was done over three residence times, 1.1 seconds, 2.3 seconds, 5.3 seconds, and two humidity ranges. The primary decomposition product seemed to be trichloronitromethane which was below $3 \mathrm{ppmv}$ in the reactor outlet. Using these data, the cost of a corona system is estimated to be significantly less than the current system being used (granulated activated carbon).

A publication will be submitted to the Institute of Electrical and Electronics Engineers on TCE/PCE destruction. Experiments for this publication are continuing, and should be completed by November 1993. TCE/PCE destruction and byproduct analysis with applied voltage and inlet concentration are complete. The remaining tests-TCE destruction with residence time, and TCE/PCE destruction with humidity-are expected to be complete early in November. Byproduct analysis will accompany these tests. 


\section{Corona for Liquid Treatment}

Experiments were performed to determine if corona was effective in degrading ethylenediaminetetraacetic acid (EDTA sodium salts). The results demonstrated that EDTA was degraded under the corona conditions. The assays for EDTA were performed using a reverse-phase ion-pairing high-performance liquid chromatography separation scheme developed in our laboratories utilizing a $\mathrm{Cu} 2+$ agent to create an ultraviolet active chromophore with the EDTA ligand. Derivitization gas ch. unatograph mass spectrometry work was done on the lyophilized extracts obtained from EDTA destruction. No oxalates or formates were found from essentially $100 \%$ degraded EDTA, however, smaller organic fragments were identified. These included nitrilotriacetic acid, iminodiacetic acid, and oxalglycine.

In the experiments above, the rate of reaction was quite slow (destruction of $>90 \%$ took over 175 hours). Concurrent efforts were focused on methods to enhance degradation rates and identify effective reactor configurations. A significantly larger, multiple electrode reactor system was constructed and tested using the decolorization of organic dyes to determine reaction rates. Initial experiments indicated that decolorization occurred at a significantly (order-of-magnitude) greater rate than previous experiments. Future experiments will provide for a more detailed analysis of destruction rates.

Conceptual ideas were proposed to suppress large streamers or arcs. Subsequent work showed that the liquid electrode alone probably provided a ballasting effect.

Publication

Laverman, L. E., G. M. Mong, L. Bettens, and S. C. Goheen. "Oxidative Treatment by Corona Discharge." Water Research, submitted.

Presentation

Goheen, S. C., G. M. Mong, M. McCullock, J. Virden, and W. O. Heath. 1993. "Organic Destruction by Corona Discharge." Presented at the AIChE National summer meeting in Seattle, Washington.

Award

A 1993 R\&D 100 award was received for the "High Energy Corona" technology. 


\title{
Process Engineering Science
}

\author{
E. O. Jones (Chemical Sciences) and M. F. Buehler (Chemical Technology)
}

\begin{abstract}
Process engineering science is the key to the application and deployment of chemical and materials processing technologies, including advanced separations, catalysis research, biomimetic coatings, and organic conversion technologies. The objective of this project was to implement and deploy materials synthesis and separation chemistry technologies; adapt existing technologies for new applications; determine the critical technical needs of the DOE and industry in chemical and materials processing; and integrate analytic, modeling, and testing capabilities to develop engineering-scale processes.
\end{abstract}

The scope of the FY 1993 effort was focused on developing a novel process that produces nanoscalesized particles and electrochemical separations. Traditionally, nanoscale (1/1000 micron) materials have not been widely used by industry because current synthesis routes do not provide the high volume necessary for manufacturers. This need was addressed by engineering a scale-up system of a laboratory apparatus that produces large volumes of nanoscale powders. Electrochemical separations, specifically electrodialysis and electrokinetics, were also studied on a laboratory- and bench-scale level to support Hanford Site separations and industrial needs.

\section{Material Synthesis}

A hydrothermal process developed at PNL that continuously produces nanoscale powders was sceled up. The process, called Rapid Thermal Decomposition of precursors in Solution (RTDS), was engineered to provide increased preduction rates. The benchscale apparatus was procured, assembled, and tested. The powders produced by RTDS have a wide variety of applications, including high-surface area catalysts, pigments for inks or paints, precursors for bulk ceramic parts, and ion exchange materials for Hanford Site separations.

Over 12 different oxides, hydroxides, and metal alloys were produced by RTDS. Some of these materials inclur iron oxides, $\mathrm{ZrO}_{2}, \mathrm{TiO}_{2}$, and $\mathrm{NiCr}$. The bench-scale unit provides a production rate 10 times that of the laboratory apparatus. The rate ranged from 1 to 5 pounds/day, a rate significantly higher than other nanoscale synthesis methods, which typically produce $\approx 100$ grams/day. In addition, a kilogram of nanoscale $\mathrm{ZrO}_{2}$ was synthesized to demonstrate the capability of producing industrialscale powder volumes.

The RTDS process was used to produce iron-based catalysts for coal conversion. The catalytic activity of the RTDS powders was significantly higher than commercially available materials. Also, consolidation studies of nanoscale $\mathrm{ZrO}_{2}$ compacts showed a decrease in sintering temperature relative to coarser micron-size material.

\section{Electrochemical Separations}

Electrochemical techniques have become increasingly popular methods to perform ion separation because they produce little or no secondary waste, operate at low temperature, and are precisely controlled by an applied potential. Electrodialysis and electrokinetics were targeted as promising candidates for broad application to Hanford and industrial waste.

Electrodialysis was used to separate aqueous mixtures of $\mathrm{Na}^{+} / \mathrm{Cs}^{+}$in support of Hanford tank waste remediation. A laboratory-flow cell was used in a threecompartment configuration. $\mathrm{Cs}^{+}$was preferentially separated over $\mathrm{Na}^{+}$by a factor of 2.5 using a commercially available Nafion ${ }^{\text {TM }} 417$ cationic exchange membrane. This result is especially encouraging because the separation can be improved further by operating in a multi-pass electrodialysis stack.
A bench-scale electrodialysis unit (electrode area of $100 \mathrm{~cm}^{2}$ ) was assembled and used to evaluate the efficiency of separating sodium nitrate. Initial experiments with Neosepta exchange membranes indi- cate a separation efficiency of $90 \%$ is easily obtained. This method is attractive because acid and base streams are generated during the salt separation, which can be used elsewhere in the remediation process. 
Electrokinetic studies were performed on Hanford soil to determine if unbound ${ }^{137} \mathrm{Cs}$ and ${ }^{60} \mathrm{Co}$ can be moved by applying an electric field. Two experiments were performed on saturated Hanford soil to monitor ionic movent in situ: a single component ${ }^{137} \mathrm{Cs}$ spike, and a binary spike of ${ }^{137} \mathrm{Cs}$ and ${ }^{60} \mathrm{Co}$. A potential of 200 volts was applied over a 10-day period, and both experiments demonstrated that unbound $\mathrm{Cs}$ and $\mathrm{Co}$ can be transported readily in Hanford soil by applying a potential. Diffusional contributions were negligible compared with migratnry and electroosmotic transport.

\section{Publications}

Buchler, M. F., D. W. Matson, J. C. Linehan, M. R. Phelps, G. G. Neuenschwander, J. L. Fulton, and R. M. Bean. 1993. "Rapid Thermal Decomposition of Precursors in Solution." R\&D Magazine, 35(11):57.

Matson, D. W., J. C. Linehan, J. G. Darab, and M. F. Buchler. "Nanophase Iron-Based Liquefaction Catalysts: Synthesis, Characterization, and Model Compound Reactivity." Energy and Fuels, in press.

\section{Presentations}

Buehler, M. F., and J. D. Norton. 1993. " $\mathrm{Na}^{+} / \mathrm{Cs}^{+}$ Separation by Electrodialysis." Presented at the AIChE 1993 National Summer Meeting.

Darab, J. G., M. F. Buehler, J. C. Linehan, and D. W. Matson. 1993. "Characterization and Processing of Sintered Products From Nano-crystalline Powders Generated by the RTDS Method." Presented at the $24^{\text {th }}$ Annual Fine Particle Society Meeting.

Linehan, J. C. 1993. "High Volume Production Methods for Nanoscale Powders," LDRD Seminar, Pacific Northwest Laboratory, Richland, Washington.

\section{Other Accomplishments}

R\&D 100 Award was received for Rapid Thermal Decomposition of precursors in Solution.

Wes E. Lawrence was hired in April from the University of Washington where he received his Ph.D. and B.S. degrees in Chemical Engineering. Wes worked on the large-scale electrodialysis experiments separating aqueous sodium nitrate salts. 


\title{
Separation Chemistry
}

\author{
R. T. Hallen (Chemical Technology)
}

This project focused research efforts on the discovery of new or improved separations of importance to the DOE mission at Hanford. Research was performed to better understand chemical separations and develop separation technologies based upon a fundamental understanding of ion exchange theory.

The main focus was the development of hybrid ion exchangers and membrane separations. Target cations for the separations included cesium and transuranic elements (plutonium and americium). Target ions of interest for other applications were identified. Of specific interest was the development of hybrid ion exchangers where a organic ligand is attached to silica gel. Alternate processing schemes were also explored. Membrane separation technologies have the potential to reduce secondary waste generation compared to the regeneration of ion exchange columns. Membrane research was directed at the development of surfactants for biomimetic membranes and the synthesis of functionalized, water-soluble polymers for ultrafiltration.

\section{Hybrid Ion Exchangers}

Hybrid ion exchangers are prepared by binding selective organic extractants to inorganic substrates. The purpose of this research was to develop synthetic capabilities at PNL for the preparation of hybrid exchangers. Silica was chosen as the inorganic substrate because of its high compatibility with glass waste forms. Extractants that are selective for cesium, technetium, and the transuranic elements separations were targeted for attachment to silica.

Two synthetic routes were explored for attachment of the organic extractants. The first approach was to anchor the extractants onto the silica surface by direct condensation reaction. Phenols are known to react with surface silanol groups undergoing condensation to silyl ethers. Hybrid exchangers are successfully prepared by this route. However, the silyl/phenol ether linkages are known to be sensitive to strong alkaline conditions, which would limit the direct use of these exchangers for tank wastes.

The second approach was to attach the extractant to the silica surface through an organosilicon bond. In the general procedure, chloro(bromoalkyl)silanes are attached to the surface through silica-oxygen-silica bonds. Extractants are then attached by nucleophilic substitution for bromide. Organosilane attachments are much less sensitive to alkaline conditions. This is the preferred route for synthesis of hybrid exchangers with much greater flexibility in attachment of organic functionality to silica.

\section{Membrane Separations}

Conventional ion exchange resins rely on specific functional groups for ion selective separations. The largest class of ion exchange resins are made from polystyrene cross-linked with divinylbenzene. The cross-linked resins are insoluble in water or solvents and swell only slightly in water. The functional groups are attached to the polystyrene backbone through covalent bonds. The groups can be charged, positive or negative, or neutral. Charged groups, such as sulfates, quartenary amines, phosphates or carboxalates, have selectivities and ion exchange capacities as a function of pH. Neutral groups, such as polyether, amine, or thiocarbonate, gain selectivity through formation of complexes or chelates with metal ions. Some dual-functional resins have also been prepared that contain both charged and chelating groups.

Functional groups can also be incorporated into watersoluble organic polymers. Improved separation technologies can be developed using the water-soluble polymers containing functional groups for metal ion specificity. The processes are based on micelles, vesicles (biomimetic membranes), and/or ultrafiltration. Organic polymers and surfactants were synthesized at PNL for evaluation. The surfactants were evaluated for vesicle formation by Professor Hal Monbouquette (UCLA). The synthesized surfactants were inferior to naturally prepared biomolecules. However, the high cost of the biomolecules makes them unattractive for commercial development. 
The use of soluble organic polymers and ultrafiltration is a viable process for industrial-scale separations. The key is to use membranes with a molecular range cutoff of 10,000 or greater to retain high flux rates. The organic polymers should have molecular weights of 30,000 or higher. The major issues with ultrafiltration are membrane fouling, membrane cleaning, and flux recovery.

\section{Other Accomplishments}

PNL collaborated with Professor Francis DiGiano from the University of North Carolina at Chapel Hill, Professor Cornelius Ivory from Washington State University, and Professor Hal Monbouquette from the University of California at Los Angeles.

The following three postdoctoral appointees were directed through the NORCUS Program: Dr. Sarah Burton, Ph.D. in Chemistry from the University of Massachusetts. Dr. Tim
Hubler, Ph.D. and M.S. in Chemistry from the University of daho. Dr. John Norton, Ph.D. in Materials Science and B.S. in Chemical Engineering and Materials Science from University of Minnesota. Dr. Burton synthesized organometallic complexes and characterized the complexes using nuclear magnetic resonance spectroscopies. Dr. Hubler synthesized polymeric ion exchangers. Dr. Norton determined $\mathrm{Na} / \mathrm{Cs}$ selectivity coefficients for membrane separations.

Dr. Sarah Burton was hired for this project.

This project partially supported Michse: Norton's M.S. thesis. Mr. Norton received his B.S. degree in Civil Engineering from Senttle University in 1988 and joined PNL in 1989. He will receive the M.S. degree in Environmental Engineering from the University of North Carolina at Chapel Hill in December 1993. His mastor's reseurch, conducted at PNL, included development of a protocol and acquisition of preliminary data for selective separation of $\mathrm{Bu}^{3+}$ using polymer-enhanced ultrafiltration. 


\subsection{Energy Systems Research}




\title{
Application of AI Technology to Power Plant Operations and Maintenance
}

\author{
D. B. Jarrell (Nuclear Systems and Concepts)
}

The objective of this project was to develop a computer-aided site assessment prototype-an automation of the decision support for operations and maintenance site characterization methodology. This prototype demonstrated the ability to assess and characterize the state of a small utility plant operations and recommend an optimized list of improvements that should be accomplished based on the cost-benefit ratio of those solutions.

The Computer-Assisted Site Assessment (CASA) project is a direct result of a generic need for an automated method of conducting site assessments identified during implementation of the Decision Support for Operations and Maintenance (DSOM) project. To provide a comprehensive, technically justifiable strategy for proceeding with a power plant improvement program, the DSOM project requires an in-depth performance assessment of the plant and its supporting programmatic infrastructure (shown in Figure 1). The cost of such an assessment normally approaches $\$ 500 \mathrm{~K}$.

The objective of the CASA project is to extensively automate such processes, thereby reducing the cost of assessments while increasing their accuracy and reliability.

In FY 1992, using LDRD funding, a methodological framework was developed showing the theory and procedural steps that would be necessary to implement the concept of a computer-assisted site assessment system.

The FY 1993 goal was to design and develop a proofof-concept prototype of the software for the CASA system.
The prototype of the software for the CASA system involved developing the functional requirements and technical specifications, and then coding the software. The CASA software prototype replicates the DSOM assessment process shown in Figure 2. First, data is collected onsite using a hand-held computer. It is then transferred to a desktop computer on a storage disk where it is related to nominal performance sto tards developed by experts. Performance indicators are then related to known degradation or failure phenomena to indicate whether a fault in the performance of a component may exist. This relationship is created using a neural network. The behavior of the neural network exhibits results like those produced by human experts.

The CASA prototype successfully illustrates a primary DSOM diagnostic assessment capability, i.e., to identify causal relationships and provide root cause directed solutions. For the prototype, an objective demonstration was designed to allow engineering verification of the concept. The CASA assessment engine can, however, be configured to assess for compliance with any well-defined objective or subjective standard (or to assess for exceptions to that standard). This allows the CASA logic to be readily adapted to any assessment task. The CASA conclusions and automated documentation were shown to form a sound technological basis for detailed diagnosis and management decision making.

Other Accomplishments

A flier giving the background and application benefits was produced and has been distributed to prospective development partners. 


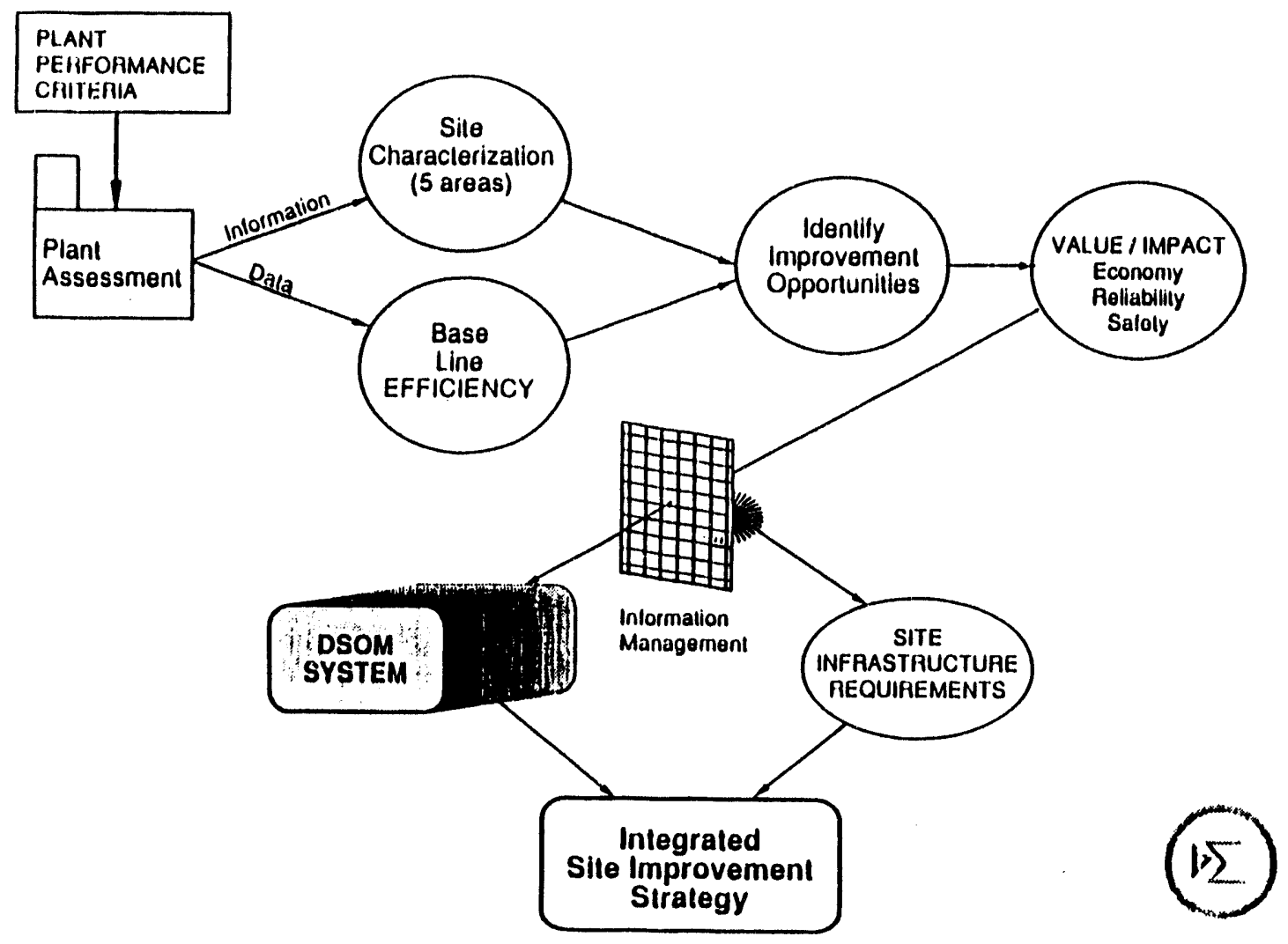

Figure 1. DSOM Plant Improvement Methodology
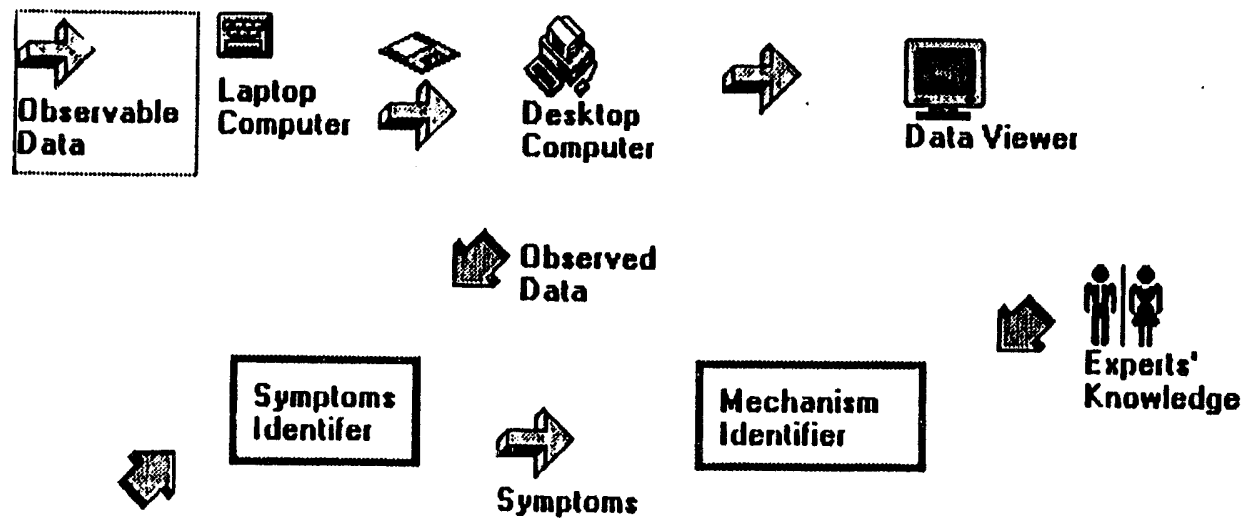

\section{Symptoms}

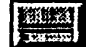

Nominal performance characteristics
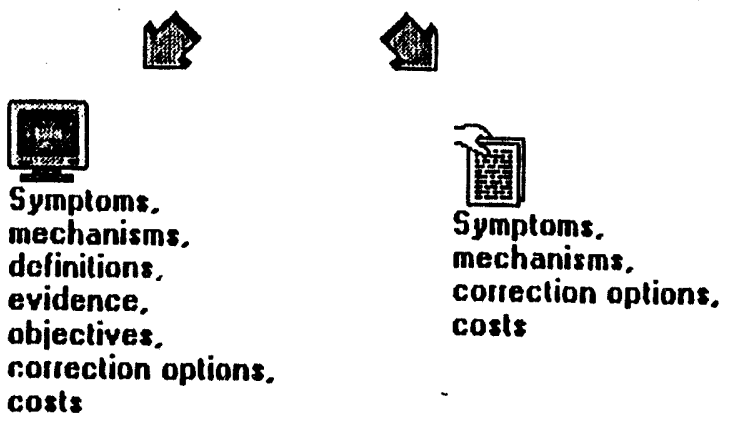

rorrection options. 


\section{Chemical Process Efficiency}

E. G. Baker, A. Y. Tonkovich, T. A. Werpy (Chemical Technology),

T. D. Brewer (Material Sciences), and M. Hoza (Computer Sciences)

The objective of this project was to acquire the scientific and processing knowledge, as well as specific tools and capabilities, required to design and develop the next generation of chemical processes. Almost all chemical processes consist of chemical reactions and separations. This project complements PNL's competency in process technology by developing new capabilities in reaction engineering. Reaction engineering is a broad topic, and we focused our investigation on the coupling of chemical reactions and separations in a single step to improve yields and selectivity of major industrial reactions.

This project has enhanced and demonstrated PNL's capabilities in the area of chemical reaction engineering, particularly with respect to separative reactions via the use of membrane reactors. Emphasis was placed on elucidating the engineering understanding required to significantly improve reactor efficiency, selectivity with respect to the desired products, or the product yield.

Improvercent of the yield and selectivity of industrial reactions is a major contribution to the development of more efficient industrial processes. By increasing materials utilization efficiency (via reducing production of low-value byproducts and wastes), efficient use of the erergy embodied in materials can be accomplished. This significantly increases the opportunity for energy savings in the industrial sector.

The technical objective was the development of a membrane reactor for alkylation reactions which employ solid acid catalysts. Solid superacid catalysts will eventually replace environmentally harsh liquid acid catalysts, but they require an efficient reactor design for implementation. While the catalyst synthesis route is discovered, simultaneous investigations into the appropriate membrane reactor design were investigated. A test reaction with a similar reaction network was chosen (the oxidative dehydropenation reaction of ethane to ethylene) and evaluated in the membrane reactor.

The membrane reactor was selected as the reactor type for several reasons. It represents a promising new field in reactor design that is capable of improving product yields per pass for parallel, parallel-series, and dehydrogenation reactions. The combination of reaction with membrane separation drives the desired reaction pathway further toward completion. Two types of membrane reactors exist and are being evaluated by PNL. The more traditional design separates a product (usually hydrogen) from reactants to overcome equilibrium limitations. The other design uses a membrane to selectively permeate one reactant into the reaction zone. This feed configuration improves the control over local reactant concentration ratios; this ratio determines the product selectivity and yield.

\section{Reaction Engineering}

A membrane reactor of the less traditional type (selective bi-molecular feed addition) was constructed and evaluated using a doped mixed metal oxide catalyst for the oxidative dehydrogenation reaction of ethane to ethylene. The performance of the membrane reactor was compared to the performance of a plug flow reactor (typical industrial design) under identical reaction wnditions. Experimental results show enhanced yields with the membrane reactor at low to moderate feed reactant ratios. At higher feed ratios (usually near the stoichiometric ratio of ethane and oxygen), the plug flow performance overtakes the membrane reactor. Ethylene selectivity in the membrane reactor was lower than in the plug flow reactor. This decrease occurs because of secondary reactions on the membrane wall. 
Theoretical investigations of the membrane reactor were done to help quantify the observed physical phenomena. A simplified model was developed to describe the reactor performance zone. The simplified model predicts enhanced performance for the membrane reactor in moderate ranges of the feed ratio. At very low ratios and at larger ratios (near or slightly greater than the stoichiometric reactant ratio) the plug flow reactor performance is predicted to approach or exceed that of the membrane reactor. A parabolic envelope of enhanced reactor performance is predicted and partially observed experimentally.

Exploratory work on innovative membrane materials was initiated focused on new dense membranes (made of nickel or other noble metals) and new or modified porous membranes.

\section{Catalysis}

Superacidic pillared lamellar materials prepared by sulfation of the metal oxide pillaring species were characterized, catalytically tested, and compared to the more typical bulk sulfated metal oxide materials which are known superacids. Both titania and zirconia pillared montmorillonite clays have been synthesized using standard pillaring procedures. Through both $x$-ray diffraction and surface area analysis, we determined that the metal oxide intercalated clays were indeed pillared (i.e., the interlayer spacing of the materials did not collapse in the absence of a swelling solvent, and the metal oxide props were laterally separated). However, the resulting catalytic activity for $\mathbf{n}$-butane isomerization was lower than that for either bulk zirconia or titania sulfated metal oxides.

We believe that the precalcination conditions for metal oxide pillared clays need to be further modified in order to account for the influence of the clay layers, and will ultimately be different than the precalcination conditions currently utilized for bulk metal oxides. In addition, the high degree of hydrophillicity of pillared clays may preclude the use of dilute sulfuric acid as a sulfating agent.

\section{Structural Modeling}

This effort focused on developing flexible modelbuilding techniques and applying them to a problem in the chemical reaction engineering area. Specific activities involved identifying relevant work in the reaction engineering and process modeling literature, developing an approach to model-building, selecting an appropriate problem area to address in a prototype, and developing a simple prototype system. The prototype, which is implemented in Prolog, demonstrates an approach for simple gas-phase systems or catalyzed gas-solid systems. Given a reaction and heats and free energies of formation of its components, the prototype balances the reaction and, at a specified temperature, determines its equilibrium constant, equilibrium conversion, and equilibrium concentrations of all species. Given concentration versus time data, the system determines the order of reaction (by comparing the concentration versus time curve with those predicted by models with different reaction orders) and plots the actual data and the predicted data for each of the candidate reaction orders.

\section{Publication and Presentation}

A paper ("Membrane Reactor/Separator: A Design for Bimolecular Reactant Addition") was prepared for presentation at the Eighth Symposium on Separation Science and Technology for Energy Applications. This paper will also be published in Separation Science and Technology. 


\title{
Electric Motor/Pump Diagnostician
}

\author{
P. J. Galatis and R. J. Meador (Nuclear Systems and Concepts)
}

This project demonstrated the ability to automate the analytical process in diagnosis of selected faults in an electric motor/pump component. This project focused on developing a pump/motor diagnostic methodology that can be coupled to other component models that demonstrate the potential for automated real-time integrated degradation and fault diagnosis. The functional specification of the logic scheme for pump/motor diagnosis will form the basis for a possible invention disclosure and the subsequent development of the software technical specification and ultimately a prototype demonstration of this new capability.

A methodology for diagnosing faults in a centrifugal pump/electric motor unit was developed in FY 1993. The methodology developed is unique in that it relates the operational characteristics and modes of performance degradation of the pump and the motor to each other and differentiates them from the system in which the pump/motor unit resides. The next step is to build the proof-of-concept diagnostician in FY 1994.

The diagnostic methodology for the centrifugal pump/ electric motor unit provides the approach and basis for developing a functional diagnostician for maximizing component efficiency and determining the source of the operational faults in a pump/driver unit. The pump/driver unit provides the diagnostician with a unique situation; two separate processes (electro/ mechanical and hydraulics) are connected by a common interface-a mechanical coupling. This set of circumstances allows for independent energy balance derivations (from both the electro/mechanical side and the hydraulic side) to be compared simultaneously with direct measurement information. This comparison provides the diagnostician with the indicators needed to form a definitive basis for the diagnosis of the centrifugal pump/electric motor unit "condition." The diagnostic model then processes this information by reducing it to a stage where a qualitative fault recognition operation can be performed. This qualitative evaluation is performed using both real time information (snap shot) and time domain (history) formats. This qualitative information from the pump/driver unit is then compared to a reference or library set of "faults" that have been characterized such that each fault is defined by a unique parametric pattern or a unique set of parametric patterns. The fault library serves two functions; it defines the specific parameters needed to diagnose a given fault condition (i.e., what information is needed to identify a particular fault) and then provides the logic (parametric) pattern or link between a preidentified fault and the "actual" condition.

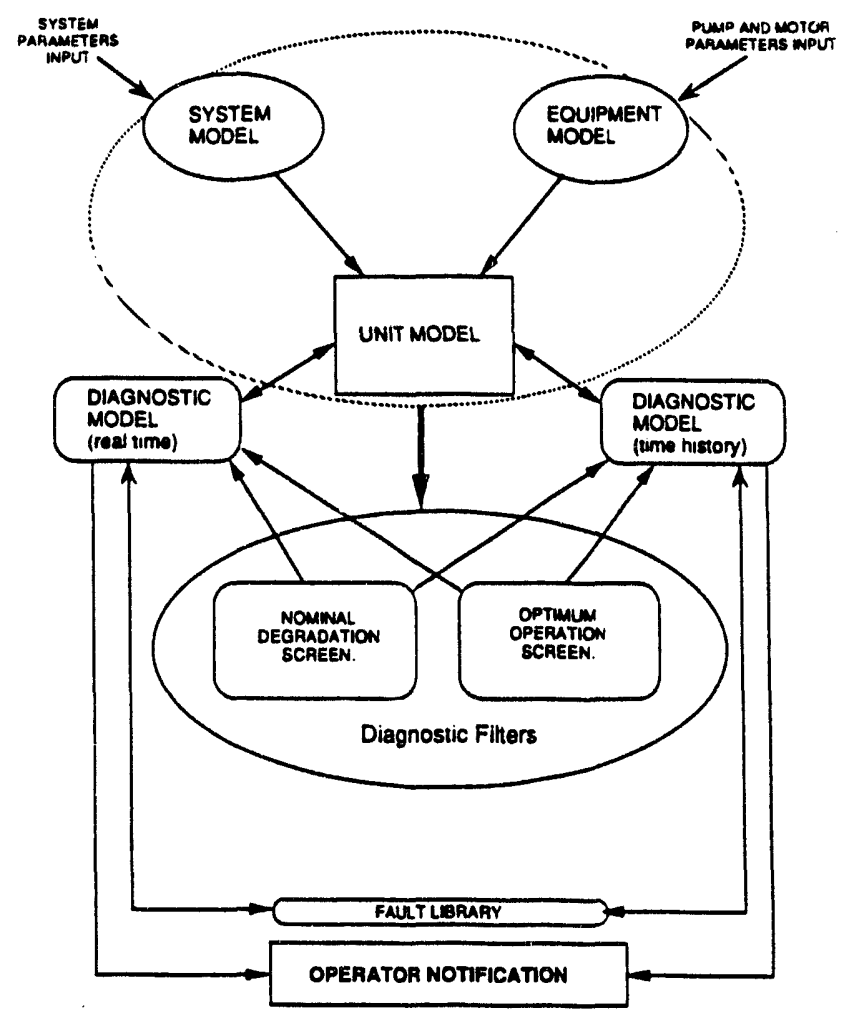

Figure 1. Pump/Driver Diagnostic Module 


\title{
General Diagnostic Reasoner
}

\author{
R. C. Stratton (Energy Sciences)
}

The technical and scientific activities of this project were to develop the diagnostic engine discrepancy analyzer and the general diagnostic reasoner methodology.

FY 1993 technical progress consisted of preparing a report (A Methodology for Developing Diagnostic Systems), software animations of the general diagnostic reasoner, and initial development of the automated diagnostic development environment.

The report presents some of PNL's research in the area of diagnosis. Specifically discussed in this document is the work pertaining to the development of a general diagnostic methodology. This methodology has been named the General Diagnostic Reasoner.

Throughout this document, diagnosis is assumed to encompass all of the following: 1) detection of an unexpected or previously defined situation, 2) identification of the physical artifact's condition in context of the situation, 3) determination of the cause for any nondesigned conditions, 4) recommendation of appropriate responses in context of the state of the diagnosis, and 5) supporting information.

The general diagnostic methodology is described in terms of hierarchical elements grouped to facilitate understanding and automation of the diagnostic process. The general diagnostic reasoner resides at the highest, or context layer of the methodology. The general diagnostic reasoner is an explicit description of diagnostic activity in the form of a general model consisting of layered processes.

At this time, general diagnostic reasoner processes are embodied in four functional layers. Each layer represents an expansion of a process element from a higher level. The processes, grouped to reflect distinct diagnostic tasks, are reduced to a level that allows them to be described as either a task-generic or domain-specific activity. The task-generic activities can be applied to any diagnostic situation regardless of the domain; the domain-specific activities require specific process information contained in the domain.

The general diagnostic methodology has not yet been fully defined. This report emphasizes the portions of the methodology that have been examined in detail and provides cursory overviews of the remaining functions. Future work will develop methodologies describing diagnostic processes not yet addressed in detail. These processes include identification of a cause, specification of the situation, development of a response to the condition, and an explanation of the diagnosis.

A scenario analysis was conducted to test the general diagnostic reasoner's robustness, identify potential problem areas, and facilitate a deeper understanding of diagnosis. The analysis consisted of implementing the general diagnostic reasoner process methodology on a heat exchanger with a single fault at the inlet water box.

To facilitate the presentation and understanding of the general diagnostic reasoner, two software animations were developed 1) animation (with pop-up explanations) of the general diagnostic reasoner methodology and 2) animation of the general diagnostic reasoner in an example diagnosis. Both of these animations are provided in Macintosh and DOS formats. The animations are user friendly and require no a priori knowledge to run.

An initial element of the software engineering environment was implemented. This element includes software to facilitate the development of the general diagnostic reasoner, knowledge acquisition, and model requirements animation. 


\title{
HVAC Diagnostic Prototype Completion
}

\author{
R. C. Stratton (Energy Sciences)
}

\begin{abstract}
Field investigations conducted by PNL have shown that poor design, installation, and operation and maintenance of energy systems can be major causes of wasted energy. This project consisted of completing a prototype heating, ventilating, and air conditioning (HVAC) system diagnostician to demonstrate the use of advanced, model-based diagnostics techniques in identifying the root cause of operating and maintenance problems.
\end{abstract}

This effort was completed over 3 years (FY 1991 through FY 1993). The following describes the activities that were undertaken:

- Identify an engineering system for prototype diagnostician development and determine the associated fault and aging phenomenon.

- Specify the capability of the diagnostician in context of the engineering system. Design and build the prototype based on the specification.

- Validate the prototype and certify it for general presentations.

In FY 1991, a number of building energy systems were evaluated for the development of a prototype diagnostician. These systems included boilers; heat pumps; chillers; heating systems; and rooftop heating, ventilating, and air conditioning units. The rooftop heating, ventilating, and air conditioning system was selected based on internal PNL expertise and the frequency of its use/application in the building industry. Additionally, typical faults and degradation modes were identified.

In FY 1992, prototype development was planned so that capabilities were developed incrementally. The plan specified the development of a no-fault diagnostician which would lead to final development of a fault-detection diagnostician. Progress was made on both of these prototypes as follows:

- The no-fault prototype demonstrates automated diagnostic capabilities during normal operation of a rooftop heating, ventilating, and air conditioning unit which has no degradation or faults in the damper subsystem. The software element that performs the reasoning, the Reasoner, is able to determine the expected and apparent heating, ventilating, and air conditioning operating mode and damper position based on process sensor information and temporal context. Additionally, the user can interact with the diagnostic system in several ways. At start-up of the process simulation and during execution, the user can specify the time of day, month of year, and manual operating mode. The no-fault prototype was completed.

- The fault-detection prototype is an enhancement to the no-fault prototype. The no-fault simulation was extended to provide simulated results of a selected set of damper faults. This prototype demonstrates automated diagnostic capabilities during faulted or degraded operation of the rooftop heating, ventilating, and air conditioning unit's damper subsystem. Additionally, this prototype provides primitive explanations to the user as to how the Reasoner element of the software determined that a fault is present. The fault-detection prototype design was completed and all software modules were coded.

In FY 1993, a fault-detection prototype was completed, validated, and made ready for presentatiuns. The prototype detects damper faults, displays a message regarding the existence of the fault, and provides an explanation of the rationale behind its reasoning. A software prototype that diagnoses a damper subsystem of a rooftop heating, ventilating, and air conditioning system was completed. 


\title{
Integration of Economic Principles with Energy/Environmental Life-Cycle Assessment
}

\author{
K. K. Humphreys (Technology Planning and Analysis)
}

\begin{abstract}
Understanding the environmental and economic impacts of the current energy production and delivery system and how these change under varying scenarios is critical to the Laboratory's ability to identify, develop, and deploy advanced energy technologies that are acceptable to the marketplace.
\end{abstract}

\begin{abstract}
A developing procedure that supports assessments of the environmental impacts of energy production and delivery systems from a "cradle-to-grave" perspective is life-cycle assessment (LCA). Currently the life-cycle assessment concept does not include economic considerations.
\end{abstract}

The objective of this project was to develop a methodological approach to integrate economics (e.g., changes in gross domestic product, gross national product, and employment) into the lifecycle assessment concept. The completed approach was to be incorporated into a computer prototype of a life-cycle assessment modeling tool.

The life-cycle assessment model (if fully developed from the prototype) would support environmentally sound technology development, waste minimization, energy conservation, and energy/economics/ environmental (3E) policy analysis.

The methodological concept selected for incorporating macro-economic indicators (e.g., employment and gross national product changes) into the life-cycle assessment concept is an adaptation of traditional economic input/output analysis. Through the application of matrix manipulation and linear algebra, input/ output analysis uses economic and related production volume data from over $\mathbf{5 0 0}$ sectors of the U.S. economy to identify macro-economic changes when the demand for a product changes (i.e., the quantity consumed is altered). As a specific example of its application, input/output analysis would help identify national and regional changes in employment, personal income, gross national product, and other macro-economic factors if a new technology/material was used to manufacture automobiles as part of a process to make them more fuel efficient. The technology/material substitution has direct labor and economic impacts which are relatively easy to identify; however, the significant ripple effects throughout the economy are not easy to identify without a method such as input/output analysis. Consider, for example, substituting composites for steel in an automobile. This results in a direct reduction in steel demand, the change in steel demand shifts activity in the fossil fuel and mineral extraction industries, and changes in mineral extraction industries impact electricity and diesel fuel markets. Similar ripple effects occur due to the increased demand for composites. Consequently, the cumulative impact of a technology/material substitution can be dramatic when its implications across a region or the nation are considered.

Whereas input/output analysis uses the underlying economic relationships between sectors of the U.S. economy, life-cycle assessment is based upon the underlying physical material and energy flows within individual unit processes and among various sectors of the economy. Through the careful development of a series of methodological concepts, the life-cycle assessment and input/output have been linked. The result is a concept that will help a technology developer/analyst who is comfortable with the details of a technology, but less comfortable with economics or policy implications, identify widespread energy/ economics/environmental implications of a technology.

Initial strides were also taken to identify PNL environmeutal impact models (e.g., air dispersion models, MEPAS, etc.) that might also be linked with the lifecycle assessment concept in order to provide further evaluation of environmental impacts and any additional economic impacts that result. 


\section{Computer Prototype Advances}

Using a simplified input/output concept, a module was coded into a Windows-based prototype of an energy/ economics/environmental life-cycle assessment model that was developed under a related LDRD effort. The module functions properly and demonstrates the concept of integrating economics with energy/ environmental life-cycle assessment. The module currently incorpurates ten sectors of the U.S. economy and would be expanded to the full 528 sectors that are typically tracked by federal agencies, such as the Department of Commerce, if the prototype is developed into a fully functional energy/economics/ environmental model through programmatic funding. 


\title{
Knowledge Acquisition Tools
}

\author{
R. C. Stratton (Energy Sciences)
}

The objective of this project was to enhance the state of the art in automated diagnostics. This includes the ability to 1) detect suboptimal performance due to improper design, operation, and maintenance; 2) identify the root causes of degradation and failures; and 3) suggest or implement corrective actions. Project efforts focused on enhancing the knowledge base in automated diagnostics. This capability includes fundamental technology that automates the process of diagnosis, a suite of tools (i.e., a development environment) for rapidly developing and testing automated diagnosticians, prototypes of selected applications that demonstrate key accomplishments, and a team of nationally and internationally renowned staff knowledgeable in this emerging field. Potential applications of this technology span a broad spectrum from small utility, building, and industrial energy technologies to consumer products.

There are a number of activities necessary for developing automated diagnostic systems. The major activities were labeled as 1) knowledge acquisition, 2) diagnostic systems design and construction, and 3) diagnostic system implementation. The principal objective of the knowledge acquisition tools task is to develop an automated tool that assists in the knowledge acquisition activity. Meeting this objective requires the following elements:

- Determining the diagnostic knowledge requirements - research to determine what knowledge is needed to do diagnosis and how it is used in the diagnostic process.
- Determining the representation requirements and acquisition methods for automating the acquisition of diagnostic knowledge - involves developing a software system specification for automating the knowledge acquisition process.

- Building the knowledge acquisition tool.

In FY 1993, technical efforts were directed toward the first element. Specifically, research was conducted pertaining to an understanding and a specification of the knowledge needed (knowledge requirements) to perform diagnosis from first principles (engineering physics). This research is documented in a report, Knowledge Requirements for Diagnosing from First Principles.

Knowledge acquisition research can be approached from a "basic issues" or an "application" point of view. The "basic issuss" perspective views knowledge acquisition as a general process, generic to all applications, and focuses on specific acquisition issues such as structured and unstructured interviews and knowledge refinement. The "application" approach analyzes the knowledge acquisition process used in the development of a number of engineering applications, and from the analysis a knowledge acquisition methodology can be developed that, at a minimum, applies to a specific domain (engineered artifacts) for a specific type of cognitive task (diagnosis). The findings reported in this document are developed from the "application" point of view. 


\section{Microscale Heat Exchanger and Heat Pump Architecture Development}

M. K. Drost (Energy Sciences) and R. S. Wegeng (Waste Systems)

\begin{abstract}
Microtechnology has the potential to dramatically change the way energy is consumed. Individual components are being developed, but before microtechnology can contribute to meeting future energy needs, microscale components must be combined into efficient and cost-effective systems. This project focused on developing the capability to design, fabricate, and test microscale energy conversion systems, such as microscale heat exchangers and heat pumps.
\end{abstract}

Systems studies were conducted to predict the performance of a range of microscale heat pump designs. The thermodynamic calculations indicated that

- Microscale heat pumps are theoretically feasible and will have performance competitive with macroscale systems if a compressor efficiency of $40 \%$ can be achieved.

- There are no apparent technical problems that would affect the technical feasibility of a microscale heat pump. Pressure drop in the microchannel heat exchangers and the use of nonchlorofluorocarbon working fluids are not significant problems.

- Performance can be significantly improved by system optimization.

Ion beam etching was used to fabricate five prototype microchannel evaporators and condensers for flow visualization. The heat exchanger channels had widths ranging between $1000 \mu$ and $50 \mu$ channel. In all cases, the channels were $100 \mu$ deep. This task successfully demonstrated the technical feasibility of deep ion beam etching in metals such as copper and aluminum.

A flow visualization loop was assembled and the prototype heat exchangers were tested. Technical accomplishments included the first demonstration of a microscale evaporator and the first visualization of microchannel phase change phenomena. Results indicate that boiling in a microchannel is a complicated process which will require further investigation.

A prototype microtechnology-based heat pump was demonstrated using an open loop cycle arrangement where the compressor was simulated by compressed refrigerant from a compressed gas cylinder. The open loop test apparatus is shown in Figure 1. To our knowledge, this was the first demonstration of a microscale condenser and a microtechnology-based heat pump. Results showed that the microscale condenser could transfer between 5 and 8 watts/square centimeter of thermal energy with a $15^{\circ} \mathrm{C}$ temperature difference. This is approximately one order of magnitude higher then required for a successful microscale heat pump. In the demonstration of the complete open loop heat pump system using microchannel evaporators and condensers, the system transferred thermal energy between high- and lowtemperature reservoirs. Refrigerant temperatures and the formation of ice on the evaporator clearly demonstrated that the system was performing as a heat pump. Figure 2 shows the heat pump evaporator after several hours of operation, with an accumulation of ice on the evaporator surfaces. 


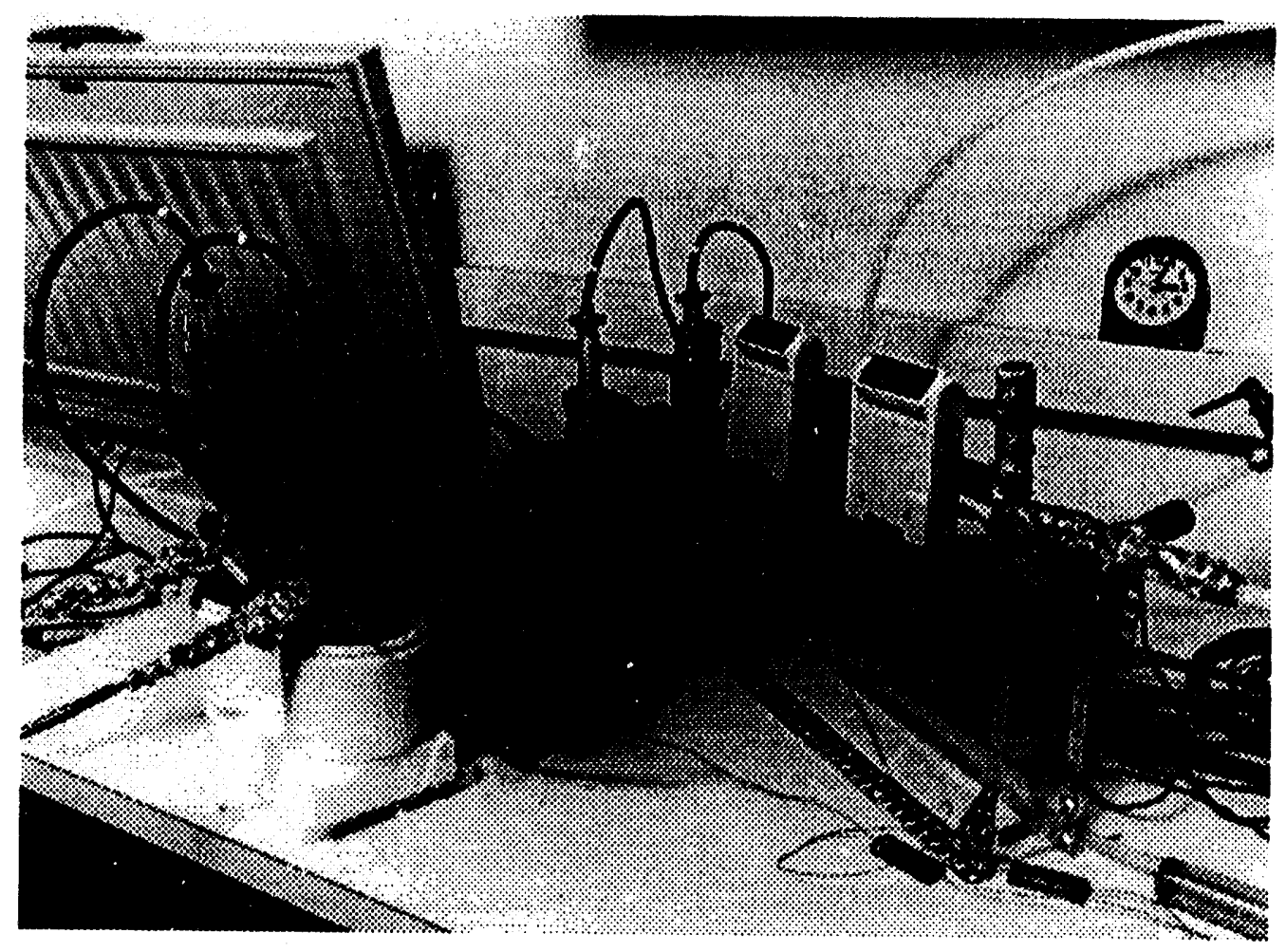

Figure 1. Open Loop Test Apparatus

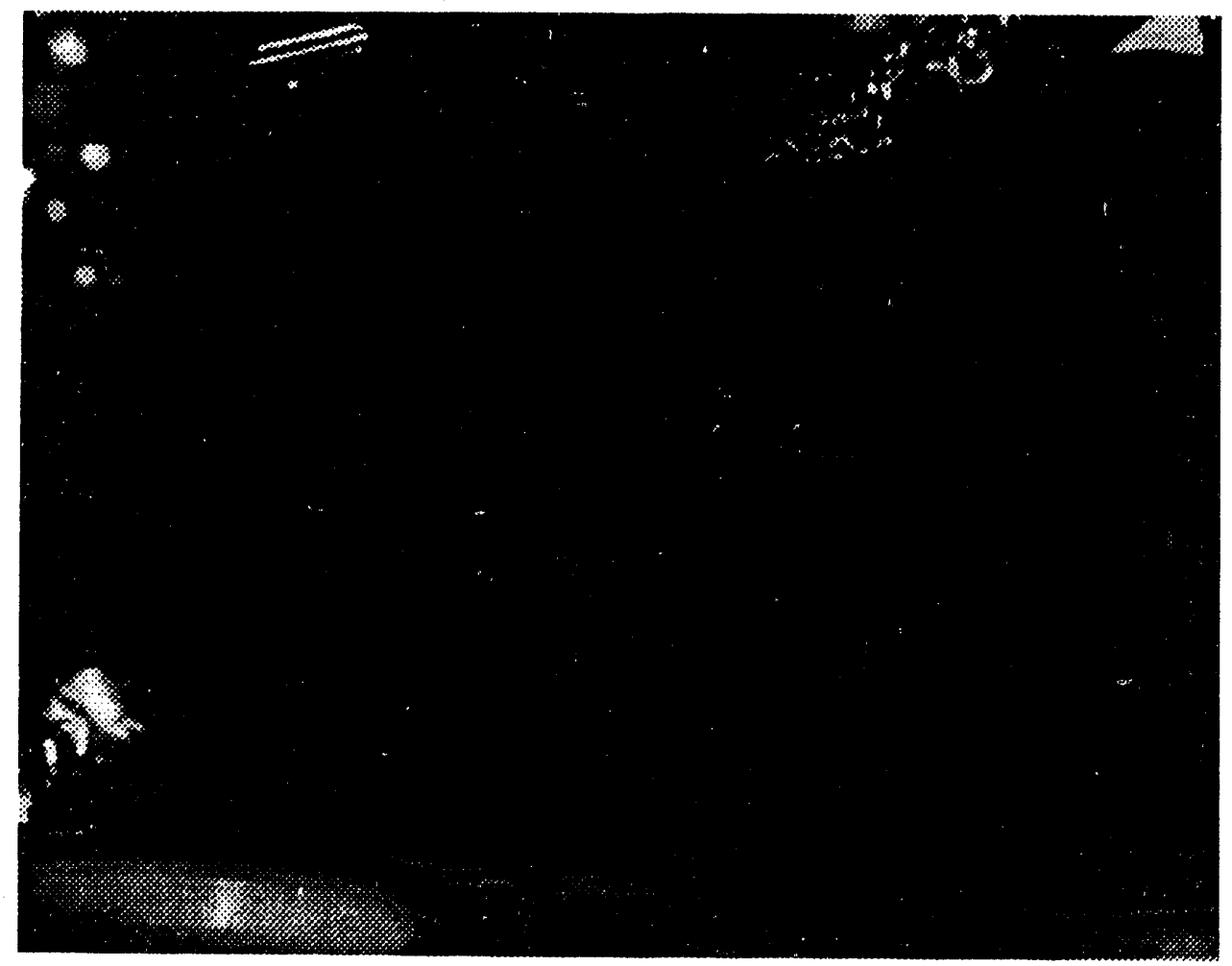

Figure 2. Heat Pump Evaporator 


\title{
Power System Dynamic Analysis
}

\author{
D. J. Trudnowski (Energy Sciences)
}

This project explored computational tools and techniques in three main areas related to the analysis and operation of next-generation power systems. The areas are

- development of measurement-based analysis techniques for modeling and understanding power system dynamics

- development of new theoretical, mathematical, and computational analysis techniques for model-based power system analysis

- investigation of the feasibility and effectiveness of using the newly developed Pulse Amplitude Synthesis and Control (PASC) technology in frequency conversion of electrical power.

\section{Measurement-Based Analysis}

Activities focused on developing fundamental mathematical algorithms for assessing a power system's dynamic characteristics from measured data.

Specific accomplishments include the following:

- Completed development and testing of an algorithm for analyzing a power system's frequency response using Chebyshev polynomials. The algorithm has several advantages for power system applications.

- Further investigated methods for model order reduction of large-scale linear systems. Techniques were analyzed to compare with the algorithm developed in FY 1992. The one developed at PNL in FY 1992 proved to be a better approach.
- Investigated Baysian analysis techniques for dynamic system analysis. The results with the Baysian work were less than promising. Although the theory of the approach is very attractive, resulting algorithms are numerically sensitive making application difficult.

- Investigated Higher-Order Statistics (HOS) analysis techniques for dynamic system analysis. An approach for assessing the stationarity of power system ambient measurements was proposed and is being investigated.

- Investigation was completed to determine bounds on the variance of a power spectrum estimation using fast Fourier transform methods.

- The commercial signal-analysis software package ADAPTICS was evaluated.

\section{Model-Based Analysis}

Development of a parallel symmetric eigensolver applicable to power system stability problems was essentially completed this year. A variety of timing experiments were done to analyze the performance of the code.

\section{Pulse Amplitude Synthesis and Control}

The objective of this activity was to demonstrate the applicability of a novel static converter technology to contemporary power system problems. In order to do this, basic research into the dynamics of the converter/power system interface must be conducted. This work is being conducted in collaboration with Montana State University where significant work in this area has been done. Computer simulation of a pulse amplitude synthesis and control system was developed to investigate effects on a power system. 
A three-phase full-scale model was developed using the electromagnetics transient computer program, and bi-directional power conversion at any power factor was demonstrated. Applications investigated include asynchronous generation and efficient ac-to-ac conversion.

\section{Field Monitoring}

The objective of this activity was to define the potential applications of synchronized monitoring systems for dynamic system analysis. Linkage was made with Bonneville Power Administration in developing a monitoring system for the western North American power system. We demonstrated how one of the measurement analysis techniques could be incorporated into the commercial package LABVIEW for direct analysis of measured system data.

\section{Visualization}

A prototype of a visualization technique for representing power system transient simulation results has been completed. The Dataviews visualization package was used to develop the representation for a generator system.

\section{Other Accomplishments}

One journal paper and one refereod conference paper were submitted for publication related to the measurement-based analyais work. The conference paper has been accepted and the journal paper is pending review. In addition, one journal paper and two conferenco papers were published in FY 1993 based on work conducted in FY 1992. A journal paper has also been submitted for the pulse amplitude synthesis and control work.

A Ph.D. student from Montana State University studying application of higher-order statistics methods to power systems spent 10 weeks at PNL.

Preliminary work was started on a journal paper describing the work done on Model-Based Analysis.

Some of these results have been compiled into a paper submitted for publication in an IEEE journal. 


\title{
Transportation Materials
}

\author{
M. T. Smith (Materials Sciences)
}

Lightweight transportation materials, advanced materials processing technologies, and low-cost coatings are required by the automotive transportation industries to meet national energy, safety, and environmental goals. This project supported DOE's efforts to address these goals through leveraging the materials research capabilities of the DOE laboratory system. This project supported the development of new knowledge and technologies for transportation applications in superplastic materials forming and coatings technology areas. Specific technical activities incorporated in the project included development and demonstration of rapid low-cost superplastic forming materials and thermomechanical processes and applications of unique wear- and corrosion-resistant coatings.

Three research areas were included in the Automotive Superplastic Forming task 1) Superplastic Forming Materials Development, 2) Superplastic Forming Modeling and Analysis, and 3) installation and operation of PNL's Superplastic Forming User Facility.

Modeling methods is an area where PNL is developing an increasing technical role which includes microstructural analysis, modeling of superplastic forming material deformation, and application of computational methods to simulate the superplastic forming process. The primary focus of this project was to support operation of the Superplastic Forming User Facility through modeling and simulation of the biaxial "butter tray" forming operation. During the year increasingly complex two- and three-dimensional models were developed that included actual superplastic forming material properties and friction conditions. Results of the modeling simulations included generation of pressure/time histories for use in user facility forming operations.

Significant progress was made on the superplastic forming Materials Development task. During FY 1993 PNL, in cooperation with Kaiser Aluminum, produced a series of nine modified Al-5083 alloys that were processed and tested at the laboratory. The results of this work included development of a lowcost alloy having vastly improved superplastic forming rates and superplastic forming elongations compared to available commercial alloys. Testing of a modified alloy containing manganese and zirconium additions demonstrated elongations of $450 \%$ at a strain rate that is more than 10 times that of current commercial alloys.

The final Automotive Superplastic Forming task was directed at establishing and operating PNL's Superplastic Forming User Facility. During the year the new Murdock $30 \times 30$-inch superplastic forming press was installed and made fully operational. Forming experiments, based on PNL modeling simulations, were conducted and destructive examination of the formed parts was used to confirm modeling predictions. In addition, the design and procurement of a new, higher pressure, gas forming system was completed.

\section{Multilayer Polymer Coatings}

The design and fabrication of the monomer pump and dispensing head for electrolyte deposition was completed during FY 1993, and the equipment was installed in PNL's web coater. Wear and corrosion resistant coatings for a number of automotive applications were developed and demonstrated. An attractive coating technology demonstrated as part of this work is diamond-like coatings. These hard, low-cost, coatings were demonstrated for scratch and wear protection on several polymer substrates, and a number of applications for the coatings are anticipated. In addition to diamond-like coatings, unique wear and corrosion resistant glassy stainless steel coating samples are currently being prepared for industry test and evaluation.

Other Accomplishments

As a result of PNL's modeling and materials development research activities, three technical papers have been submitted for upcoming conferences. These include the Fall meeting of The Metallurgical Society (Pittsburgh, Pennsylvania, October 18-21, 1993) and the International Superplasticity Conference (ISCAM 94, Moscow, Russia, May 1994). 
To support the developing Superplastic Forming programs, two

Ph.D. now hires were made during FY 1993. These include
M. A. Khaleel in the Applied Physics Center and J. S. Vetrano in the Materials Sciences Department. 


\subsection{Other Laboratory-Level Projects}




\title{
International Germanium Experiment
}

\author{
R. L. Brodzinski (Chemical Sciences)
}

The measurement of the double-beta decay of ${ }^{76} \mathrm{Ge}$ is considered by most physicists to have the highest potential for determining the correct form of the Grand Unified Theories of physics. We currently have designed and built the most sensitive experiment in the world for making this measurement, but it is not sufficiently sensitive to clearly resolve the nuclear structure parameters in question. The only solution to this dilemma is to fabricate low-background detectors from enriched germanium, which is not available in the United States.

Earlier efforts in this program resulted in the fabrication of three 1-kilogram detectors from 5 kilograms of $87.44 \%$ enriched ${ }^{76} \mathrm{Ge}$ supplied to us by the former Soviet Union. We have made the first confirmed measurement of the two-neutrino double-beta decay of ${ }^{76} \mathrm{Ge}$ and the first measurement of the double-beta decay of ${ }^{100} \mathrm{Mo}$ to the first excited $0^{+}$state of ${ }^{100} \mathrm{Ru}$.

An international consortium, the International Germanism Experiment (IGEX), has been formed including PNL, the Universities of Minnesota, South Carolina, and Zaragoza, Spain; the Institute for Theoretical and Experimental Physics in Moscow; the Yerevan Physical Institute; and the Soviet Academy of Sciences' Institute for Nuciear Research (INR), to produce several more germanium detectors enriched in ${ }^{76} \mathrm{Ge}$. The goal of PNL and the International Germanium Experiment is to measure, or place meaningful limits on, the zero-neutrino double-beta decay mode of ${ }^{76} \mathrm{Ge}$ for resolution of Grand Unified Theories. In order to achieve that goal, it will be necessary not only to produce large, highly enriched germanium detectors, but also to ensure that the radioactive background in the region of interest, $2038.56 \mathrm{keV}$, the Q-value for the decay, is virtually zero so that any neutrinoless double-beta decay events can be unequivocally identified. During the past year, we identified several new sources of background and devised methods to eliminate them. For example, the electroformed copper components of the spectrometer system were found to contain ${ }^{226} \mathrm{Ra}$ and ${ }^{228} \mathrm{Ra}$ contamination at levels of 3.5 and $1.3 \mathrm{mBq} / \mathrm{kg}$, respectively. A barium sulfate scavenge added to the electroforming bath filtration system reduced these radium contaminants more than a factor of 20 . Similarly, a ${ }^{228} \mathrm{Th}$ contamination of $0.12 \mathrm{mBq} / \mathrm{kg}$ in the copper has been identified, and it is hoped that use of high purity sulfuric acid and recrystallized copper sulfate in the electroforming baths will eliminate this thorium contamination. To put this in perspective, this measured thorium contamination amounts to 10 atoms of ${ }^{228} \mathrm{Th}$ per gram of copper or about 1 part in $10^{21}$. Material purity requirements at these levels are clearly out of the ordinary.

Our Russian colleagues have delivered $\approx 15$ kilograms of enriched germanium $(\approx \$ 30,000,000$ worth) to the United States for fabrication into ultralow-background detectors by PNL. The first crystal has been pulled from this material, and a single detector weighing 2.190 kilograms has been fabricated from this crystal. This detector has been installed in the experimental enceinte in the Homestake mine in South Dakota. Data are currently being acquired. At least five such large detectors are planned within the next few years.

A collaboration has been initiated that includes PNL and the Universities of South Carolina; Zaragoza, Spain; and Buenos Aires, Argentina to establish a "cosmion" experiment in the southern hemisphere. The design takes advantage of the maximum variation in the thickness of earth available in the southern hemisphere for an experiment observing diurnal modulations of interactions engendered by cold dark matter candidates in the galactic halo. The goal of the experiment is to elucidate potential candidates for the missing (nonluminous) mass of the universe. An experimental site is being prepared in an iron mine in Sierra Grande, Argentina. The cosmion detector has been designed, and fabrication is scheduled to begin in January 1994 using programmatic funding.

\section{Publications}

Brodzinak, R. L. :993. "Applying High-Energy Physics Instrumentation to Enyironmental Restoration." Bull. Am. Phys. Soc. 38:1800. 
Brodzinski, R. L., F. T. Avignone, J. I. Collar, H. Courant, E. Garcia, C. K. Guerard, W. K. Hensley, I. V. Kirpichnikov, H. S. Miley, A. Morales, J. Morales, R. Núñez-Lagos, S. B. Osetrov, V. S. Pogosov, A. A. Pomansky, J. Puimedón, J. H. Reeves, K. Ruddick, C. Sáenz, A. Salinas, M. L. Sarsa, A. A. Smolnikov, A. S. Starostin, A. G. Tamanyan, S. I. Vasiliev, and J. A. Villar. 1993. "Status Report on the International Germanium Experiment." Nucl. Phys. B (Proc. Suppl.) 31:76.
Collar, J. I., F. T. Avignone, III, R. L. Brodzinski, E. García, H. S. Miley, A. Morales, J. Morales, R. Núñez-Lagos, J. Puimedón, J. H. Reeves, C. Sáenz, A. Salinas, M. L. Sarsu, and J. A. Villar. 1993. "Remarks on Direct Searches for Cold Dark Matter Candidates." Nucl. Phys. B (Proc. Suppl.) 377. 


\title{
International Reactor Safety
}

\author{
D. F. Newman (Nuclear Systems and Concepts)
}

The objectives of this project were to develop an enhanced and insightful understanding of the safety needs of the nuclear power reactors designed by the former Soviet Union, in order to enhance the technical capabilities necessary to address these needs.

The focus of the activity was directed at the graphite moderated, water cooled, pressure tube $R B M K$ reactors. This focus is appropriate because it is in this type of reactor that Hanford can offer unique capabilities and experience. Also appropriate to the Hanford experience, is assistance in the adaptation of Russian military production reactors, which have a dual power and weapons material production role, to a purely civilian application. The Russian military production reactors have significant similarities to the Hanford production reactors. In addition, this project was also applied to specific areas such as operational safety of the pressurized water VVER reactors.

\section{RBMK Loss-of-Coolant Accident Modeling}

A significant area of safety to be addressed concerns loss-of-coolant accidents (LOCAs) for RBMK (Chernobyl-type) reactors. PNL has access to the only U.S. expertise for graphite-moderated, watercooled, channel type reactors, and the REL, IP5/ MOD3 computer code that has been used for loss-ofcoolant accident analysis of U.S. commercial reactors and DOE reactors such as the Hanford N-Reactor.

A RELAP5/MOD3 model of an RBMK reactor has been developed for evaluating a limited set of RBMK loss-of-coolant accident events. This set of events are single or multiple fuel tube ruptures. The initial phase of this development was to establish the applicability of RELAP5/MOD3 for the RBMK boiling water reactor. RELAP5 has been extensively applied to U.S. commercial light-water moderated, pressurized water reactors (PWRs), and also to the NReactor graphite moderated reactor. However, it was considered necessary to review its applicability to the
RBMK graphite moderated, boiling water reactor (BWR). It was quickly concluded that the RELAP5 neutronics package would not be able to accurately predict the core behavior for the large, loosely coupled core of an RBMK, however, fur tube rupture and other small loss-of-coolant accident events, this was deemed to not be a limiting concern. It was also concluded that the heat transfer and fluid dynamics packages were in general applicable to the RBMK design, and in particular, to loss-of-coolant accident events. Although potential problems were identified with the default critical heat flux correlations and counter current flow model with respect to the RBMK fuel design, the general heat transfer and fluid dynamics packages were acceptable. A prime concern in this area was the choke flow model. To resolve this, benchmark cases were made to verify the choke flow model, which was concluded to be satisfactory. Thus, although there were potential modeling limitations identified, it was concluded that RELAP5/ MOD3 could be used to analyze the set of loss-ofcoole ccidents events that were envisioned.

Available detailed information on the RBMK design was limited, so model developinent concentrated on providing an accurate fuel region and steam separator model. The fuel model would allow reasonable prediction of the fuel cladding response. The steam separator model was considered important for accurately predicting both total coolant inventory and the fluid properties for the reverse flow that would occur for the affected (ruptured) tubes.

\section{Loss-of-Coolant Accident Analysis for RBMK}

Two types of loss-of-coolant accident events were investigated for the RBMK reactor. These were a single and dual tube rupture at the core inlet, and an inlet flow blockage to a single tube, similar to the Leningrad tube rupture event. The inlet single and dual tube rupture event was investigated for the fuel temperature and reactor inventory response. General conclusions reached were that initial fuel in the affected tube(s) was not a concern, but that a prolonged loss of coolant inventory presented some unique challenges to ensuring extended reactor core flow for the entire core. The inlet tube blockage 
analysis was set up to simulate the Leningrad tube rupture event, and to provide a parametric study of the inlet valve that had failed at the Leningrad reactor. Several cases were evaluated that varied the percent area assumed to be blocked for the inlet valve. The purpose of this evaluation was to determine the time at which tube rupture would occur following the blockage. The results of this analysis are shown in Figure 1. These calculation results compared against calculations presented to PNL by Drs. O. Novoselsky and S. Kuznetsov (RDIPE, Moscow - chief designers for the RBMK), and were found to be in very good agreement.

\section{Steam Void Reactivity Effect in RBMKs}

The reactivity effect of increasing steam voids in RBMK reactors is the net result of positive contributions from the $\mathrm{UO}_{2}$ fueled channels, and negative contributions from supplemental absorber and control rod channels. A thermal-hydraulic transient that initiates voiding of fuel channels will be propagated to these absorber channels in a delayed fashion because of the much lower heating rate and feed water flow rate in these channels. Reactivity transient analyses performed on RBMK reactor cores have been based on simultaneous steam void propagation in all channels. This synchronous steam void assumption is only valid for very slow transients. For larger reactivity transients, it is necessary to couple the thermalhydraulic behavior in different types of channels together from a RELAP analysis to three-dimensional neutronic core analyses for simulation of the reactivity feedback effects. The time constant for thermalhydraulic steam voiding transients is an important safety parameter for avoiding beyond-design-basis accidents in RBMK reactors, since the $\mathrm{UO}_{2}$ fueled channels have positive reactivity contributions from steam voiding, as shown in Figure 2. The coolant void reactivity feedback is reduced by increasing the fuel enrichment from 2.0 to $2.4 \mathrm{wt} \%{ }^{235} \mathrm{U}$, primarily due to the accommodation of 80 additional absorber channels in the core.

Use of plutonium fuel in the RBMK core will eliminate the positive steam void reactivity effects in the fueled channels, but will reduce the delayed neutron fraction. The extent that this will limit available excess reactivity for normal operations, and change the xenon stability behavior and control system worth for the core, require analysis.

\section{Publications}

Meriwether, G. H., and J. P. McNeece. 1993. Flux Stability and Power Control in the Soviet RBMK-1000 Reactors. PNL-8781, Pacific Northwest Laboratory, Richland, Washington.

Newman, D. F. 1993. Russian RBMK Reactor Design Information. PNL-8937, Pacific Northweest Laboratory, Richland, Washington.

\section{Presentations}

Tsiklauri, G. V., and B. E. Schmitt. "RELAP5/MOD3 Code Assessment for Pressure Tube Graphite-Moderated Boiling Water Reactor," accepted for publication in proceedings and presentation at the International Conference on New Trends in Nuclear System Thermohydraulics to be held at Piza, Italy, on May 30 to June 2, 1994.

\section{Other Accomplishments}

One new scientific/engineering staff member, Dr. G. Tsiklauri, was hired full-time to work on this LDRD project during FY 1993. Dr. Tsiklauri was formerly a member of the Russian Academy of Science.

The Russian scientists and engineers were very impressed with the adaptation nuade by PNL with RELAP5/MOD3 to predict the thermal-hyciraulic behavior in an RBMK during loss-ofcoolant accident events and severe accidents. Director of RDIPE, Dr. O. Adanov and Deputy Director, Dr. Y. Cherkashov addressed the U.S. Department of State and DOE for assistance in the project "Studying Applicability of RELAP5/MOD3 Code for RBMK-1000 and RBMK-1500" and requested DOE funds for this support. Personnel from NIKIET have supplied some transient data from the Leningrad incident, additional controls for their confinement, piping upgrades, and reactor cavity pressure response. PNL is accumulating data from NIKIET, INS, Kurchatov, Atomoerge project, and VNIIAES, and has several small contracts to provide additional data. The results from former Soviet Union experiments and analyses will be added to the data base as the information becomes available. 


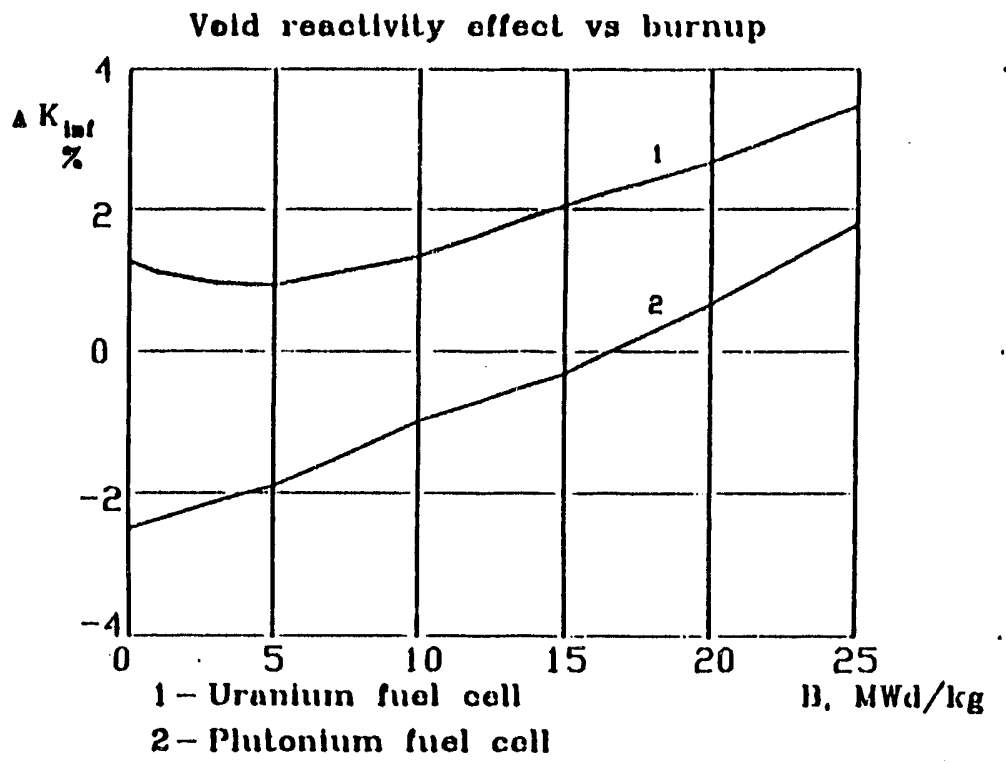

Figure 1. Inlet Flow Blockage W/Slow Scram Time to Tube Failure After Blockage

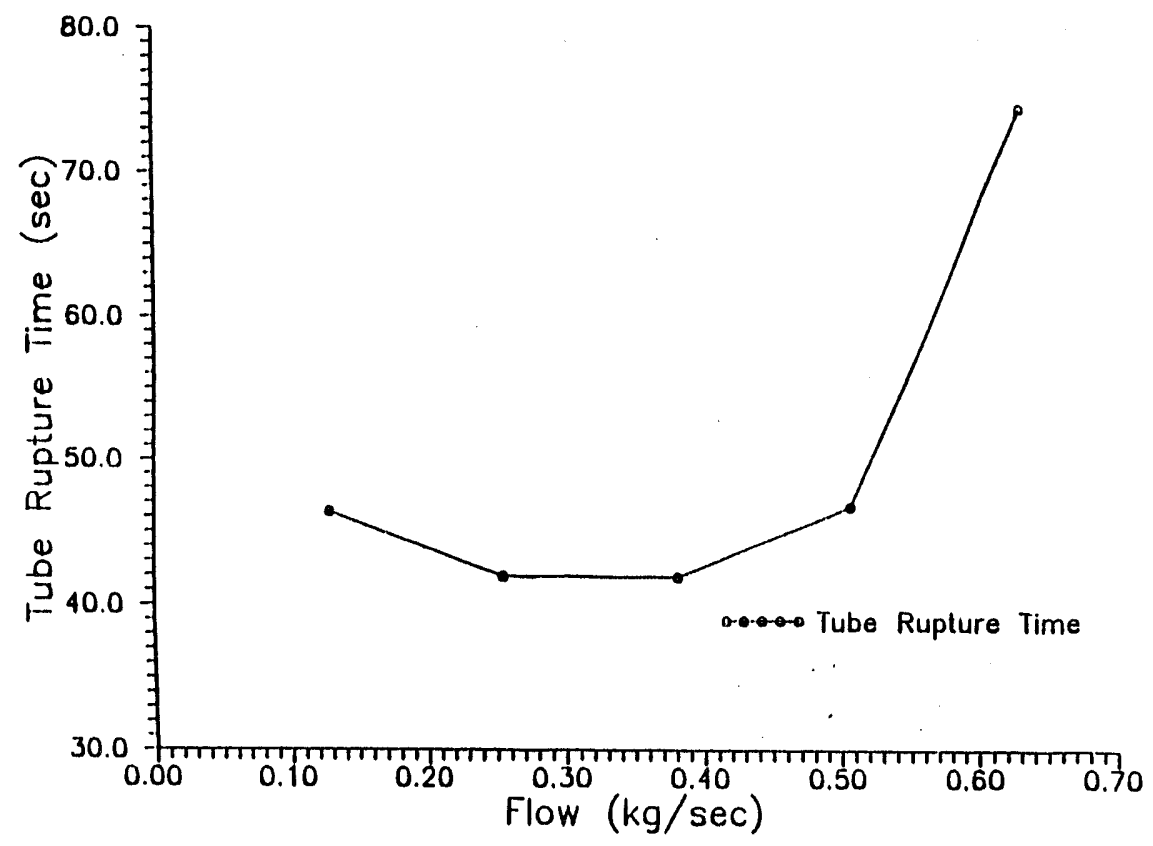

Figure 2. RBMK Results from NIKIET 


\title{
Weapons-Grade Plutonium Disposition
}

\author{
D. F. Newman (Nuclear Systems and Concepts)
}

The objectives of this project were to develop an enhanced and insightful understanding of the disposition options for surplus weapons-grade plutonium, enhance the technical capabilities to address these options, and to interact technically with Russian scientists on the feasibility of concepts proposed for burning weapons-grade plutonium. The focus of the activity was directed at exploring the feasibility for U.S. and Russian light water reactor technology to use surplus plutonium as fuel in order to denature the weaponsgrade material to reactor-grade. Non-fertile plutonium fuel concepts can double the rate of plutonium denaturing in comparison to conventional mixed oxide fuel. This project developea' technical bases and methods for evaluating the feasibility of non-fertile fuels, and compared their development needs and schedules with mixed oxide fuel options.

\section{Fuels Burnup Model Advances}

The basic vehicle for demonstrating capability for analyzing the burnup characteristics of weapons-grade plutonium for disposition as spent fuel is a typical U.S. pressurized light water reactor model.

Due to high fabrication costs for plutonium fuel, in comparison to commercial $\mathrm{UO}_{2}$ fuel, high fissile plutonium loadings are desirable to minimize disposition costs for weapons-grade plutonium. The addition of burnable neutron absorbers in the fuel is required to reduce the excess reactivity of the fuel to a range that can be accommodated by existing control rod systems. The recent licensing of erbium in commercial $\mathrm{UO}_{2}$ fuels by the Nuclear Regulatory Commission was the basis for extending plutonium fuel burnup models to include erbium as the burnable absorber. Since natural erbium contains four major isotopes, the model includes the burnup chains among the isotopes. The capabilities of the burnup model in the WIMS-E code to accurately predict reactivity changes due to a wide range of erbium loadings was verified with continuous-energy Monte Carlo methods (using the MCNP code) with the most recent evaluated nuclear data files (ENDF/B-6).

\section{Disposition of Weapons-Grade Plutonium as Spent Light Water Reactor Fuel}

Assessments were performed of the capabilities of a typical U.S. light water reactor to denature weaponsgrade plutonium to fuel-grade while displacing conventional commercial fuel using fuel in the form of a $\mathrm{UO}_{2}-\mathrm{PuO}_{2}$ mixed oxide or a non-fertile fuel form. A paper describing the results of this work, "Burning Weapons-Grade Plutonium in Reactors" PNL-SA21828, was presented at the 4th Annual Nuclear Society Conference in Nizhni Novgorod, Russia, on June 30, 1993, and published in the proceedings.

\section{Options for Weapons-Grade Plutonium Use in Russian Reactors}

Assessments of the capabilities of Russian reactors to use weapons-grade plutonium fuels to improve their safety characteristics while denaturing the plutonium to reactor-grade in the spent fuel were initiated. An understanding of the behavior of mixed oxide fuels in commercial graphite-moderated boiling water-cooled Russian reactors (RBMKs) and non-fertile plutonium fuels in graphite-moderated water-cooled Russian production reactors was obtained.

Publication

Newman, D. F. 1993. "Burning Weapons-Grade Plutonium in Reactors." In Proceedings of the 4th Annual Nuclear Society Conference, Nizhni Novgorod, Russia. 


\subsection{Center-Level Projects}




\subsection{Applied Physics}




\title{
Direct Numerical and Large Eddy Simulation of Turbulent Fluid Flows
}

\author{
J. R. Phillips (Analytical Sciences)
}

The objective of this project was an initial study on the development of direct numerical and large eddy simulation of turbulent fluid flows. Enhancement of capabilities in this research area is encouraged since these computational fluid dynamics algorithms have become common in the research literature and may become important predictive tools within the next decade.

Direct numerical simulation techniques were used to perform initial transition and turbulence calculations of the unstratified double pane window flow problem. The numerical algorithm has been implemented on the Intel Touchstone Delta architecture and exhibits satisfactory parallel performance.

Three different test cases were considered as initial tests of the code. The first case was a decay of a "gray" noise perturbation of the conduction regime back to a quiet conduction regime.
The second case considered a supercritical growth of an oblique disturbance into a steady cellular convection state. Apparently, transverse disturbances in the oblique wave may grow rapidly to become a dominant part of the transition becween the steady onedimensional conduction regime and a steady multidimensional cellular state. This result has not been previously computed to my knowledge. Further calculations will have to be performed to validate the existence of this type of transition. The third case is a supercritical transition to a turbulent convection state and an examination of the turbulence statistics of the flow. Further turbulance simulations are necessary to study the effect of $\mathrm{Gr}_{\text {. }}$.hof number and Prandtl number on the turbulence statistics.

\section{Publication}

An abstract has been submitted for publication in the American Physical Society bulletin and a presentation of this material was given at the November 1993 American Physical Society meeting. 


\title{
Electrolyte Ballasting for Parallel Discharge Systems
}

\author{
D. L. Lessor (Analytical Sciences)
}

In FY 1993, the concept of electrolyte ballasting for equalizing currents and suppressing arcs in pa: allel corona discharge systems was investigated by 1) a search of literature and paten for previous experience with the concept, 2) a secirch of the literature for evidence of response characteristics of electrolyte conduction in fast, high current electrical pulses, 3) an investigation of implications of standard theories of electrolytic conduction and electrolyte polarizability on a fast time scale, 4) a back-of-envelope design of a system, based on required steady state current levels and the required limits on the size of fast current pulses, and 5) if promising, rough design of an experiment to test the concept. Products of the investigation should include an assessment of the validity and promise of the concept, and if promising, a plan for verification tests.

We found both theory (Chang and Jaffe 1952) and experiments (Jaffe and Rider 1952; Scott et al. 1967) reported in the literature confirming that an electrolyte-electrode interface should resist large unidirectional current surges, essentially because transport and reaction rate limits result in a pileup of electrochemical reaction products or deficiency of reactants at the interface. With ac driving, this pileup phenomenon manifests itself in an increased resistance as driving frequency of a voltage applied to an electrochemical cell approaches zero, which is one of the phenomena reported in the literature that we take as supporting our conclusion. In ac, the pileup phenomenon occurs only if there is sufficient time in a half cycle. One aspect of this phenomenon is different than we expected. The ions near the interface can respond to very fast small pulses by small relative displacements of positive and negative charge. The current limiting effect comes because a predominantly unidirectional pulse causes ion or molecule pileup and/or deficiency. Thus, the phenomena at an electrode-electrolyte interface could serve to limit large pulses whose time scale is sufficiently long to cause the ion pileup or deficiency phenomenon, but not very short pulses nor high frequency oscillatory noise.
A literature search turned up no evidence that this phenomenon had been deliberately used to control current surges or to achieve ballasting (stabilization and equalization) of parallel discharges. Order of magnitude relations which we have deduced between pulse durations for which the phenomenon is effective and the needed thickness of the electrolyte layer should give some guidance in determining potential applications, but we have not positively identified these applications.

We did find, however, that one of the large-scale commercial applications which we had envisioned, that of stabilizing parallel discharges in nitrogen fertilizer production, is not applicable tecause nitrogen fertilizer is mostly produced by refined versions of the Haber-Bosch process and not by the electrical discharge Birkeland-Eyde process as we had thought.

Suppression of arcing in an electrostatic precipitator would appear to be one possible application. Here one would not necessarily need to prevent ares. Thus, one would not need to shut down the current on a time scale of streamer transit or streamer-to-arc transition, but rather to shut down arcs on a time scale short compared with their natural duration or time to equipment failure, and at a cost and reliability superior to that of havivare voltage interrupt systems. An electrolyte ballast on each electrode wire could interrupt a current $\mathrm{e}$ there, obviating a momentary interrupt of the voltage supplied to an entire array of electrode wires.

The theoretical work of Chang and Jaffe (1952) showed the importance of two dimensionless parameters in the frequency response of an electrodeelectrolyte-electrode sandwich, the parameters being $\rho$ $=\xi 1 / D$ and $\lambda=1(\varangle / 2 D) .^{1 / 2}$ Here $\xi$ is a reaction rate constant (the number of electrochemical reactions for a species at the electrode per unit time per unit electrode area per unit of excess concentration of the species above the steady state zero current value), 1 is the thickness of the electrolyte layer, $\boldsymbol{z}=2 \pi f$ is the angular frequency, and $D$ is the diffusion coefficient for the species. The theory of Chang and Jaffe can be used to show that for the effective conductance $1 / R$ to 
differ significantly from the value $1 / \mathbf{R}_{\infty}$ at infinite frequency (at which no ion pileup effects occur), it is necessary to have $\lambda \leq 1$. This can be re-expressed as

or

$$
\text { ఒ } \leq 2 \mathrm{D} / \mathrm{1}^{2}
$$

$$
\mathrm{T} / 2 \geq \pi l^{2} /(2 \mathrm{D})
$$

In making the prescription from ac to a de pulse, we expect that the amount of ion pileup or deficiency which occurs in a voltage half-cycle of duration $T / 2$ is approximately that in a pulse of duration $t_{\text {pulse }}=(2 / \pi)(T / 2)$, we obtain the criterion that a voltage pulse will have an impeded current response if

$$
t_{\text {pulse }} \geq 1^{2} / D
$$

Because the diffusion coefficient $D$ for common electrolyte ions in water is on the order of $D=1.5 \mathrm{x}$ $10^{-9} \mathrm{~m}^{2} / \mathrm{sec}$, an electrolyte layer of thickness $1=$ $10^{-5} \mathrm{~m}$ would be needed to impede pulses of 0.07 seconds and longer, and even impeding pulses down to 1 second (while letting significantly shorter ones pass) would require a layer of thickness $1=4 \mathrm{x}$ $10^{-5} \mathrm{~m}$, which is roughly the thickness of moderately thin paper. Hence one could consider an electrolyteimpregnated paper for the electrolyte layer in the electrode-electrolyte-electrode sandwich.

Thus, only a small device is needed to inhibit long unidirectional pulses, but undoubtedly some problems accompany this miniaturization. The ion pileup phenomenon essentially applies a multiplier to the infinite-frequency impedance $R_{\infty}$. For effective ballasting (current stabilization) against current surges and effective drop in the load resistance, as occurs when a discharge tries to make the transition coronato-streamer-to-arc, one expects the ballast electrolyte sandwich to provide a normal operating resistance, then to supply a higher resistance (with a new level of pileup achieved) when the current tries to make a transition to a much higher value. The baseline resistance of the electrolyte ballast, however, would probably need to be a non-negligible fraction of the load resistance [i.e., the load voltage-to-current operating ratio $\left(\mathrm{V} / \mathrm{I}_{\mathrm{op}}\right]$ ]. The electrolyte ballast resistance vrith the new ion-pileup-multiplier should then be siguificantly gneater than the load resistance. The requirements are then

$$
\begin{aligned}
& {[1 /(\sigma A)] M_{1}=h(V / I)_{\text {op }}} \\
& {[1 /(\sigma A)] M_{2}=h^{\prime}(V / I)_{o p}}
\end{aligned}
$$

where $M_{1}$ and $M_{2}$ are the multipliers supplied to the electrode-electrolyte-electrode sandwich resistance by the operating and the new (current surge) levels of ion pileup, and $h$ is a value less than or on the order of 1 , and $h^{\prime}$ is a value greater than 1 . Here $[1 /(\sigma A)]$ is the infinite-frequency resistance of the electrolyte, with $\sigma$ its conductivity and $\mathbf{A}$ the area available for current flow. (In experiments in which the electrolyte thickness 1 was varied, the added resistance for a given frequency, concentration, and electrode geometry was found to be somewhat independent of 1 . Making the electrolyte layer thickness thin according to the prescriptions given above ensures that the ion pileup region has a major influence on the total resistance, thus permitting large values of the multiplier which we have designated $\mathbf{M}_{2}$.) If the conditions of the two preceding equations can be met, the electrolyte ballast should offer better current surge suppression and/or less resistive energy dissipation than a simple resistor in series with the load.

A second requirement for the inhibition action against current surges for an electrode-electrolyte-electrode sandwich is that the electrodes be sufficiently inert. This is embedded in the theory (Chang and Jaffe 1952) in the dependence on the parameter $\rho=\xi 1 / D$ described above, and it is verified experimentally (Jaffe and Rider 1952). Thus, Pt, Au, Ni, and $\mathrm{Al}$, in that order, give decreasing levels of low frequency current inhibition because of decreasing electrode inertness (Jaffe and Rider 1952). Inert electrodes have low reaction rates, which correspond to low values of $\rho$, which in turn give rise to large amounts of ion pileup. Graphs of the theory of Chang and Jaffe suggest that if an inertness parameter as low as $\rho=0.3$ could be achieved, then an effective resistance multiplier on the order of 8 could be achieved at a value $\lambda=0.6$, where $\lambda=1(\omega / 2 D)^{1 / 2}$ as described earlier. With our prescription from ac driving to voltage pulses, this corresponds to supplying eight times the infinite-frequency resistance to pulses whose duration is longer than puleo $\geq 2.8 \mathrm{I}^{2} / \mathrm{D}$ and some suppression to pulses as short as $t_{\text {pulse }} \geq 1^{2} / D$. With an electrolyte layer of the thickness of a piece of paper ( 2 mils, or $5 \times 10^{-5} \mathrm{~m}$ ), one could strongly suppress voltage surges after 2 seconds, and very strongly after 5 seconds. The data of Jaffe and Rider (1952) is consistent with a value $\rho=0.3$ for a goldplated electrode in contact with $\mathrm{KCl}$ (potassium chloride) solution at molarity $10^{-2}$.

The design of the proposed surge suppression device would have the electrode-electrolyte-electrode 
sandwich as its basic element, with the thickness of the electrolyte chosen for the minimum duration of the pulses one wants to suppress. One would choose the resistance by choosing either the area $A$ available to current flow or the number of sandwich configurations in series. The entire configuration could probably be encapsulated for ruggedness and moisture retention like a simple resistor. Some attention would need to be given to heat dissipation for the intended current levels.

\section{References}

Chang, H. and G. Jaffe. 1952. "Polarization in Electrolytic Solutions. Part I. Theory." Journal of Chemical Physics 20(7):1071.

Jaffe, G. and J. A. Rider. 1952. "Polarization in Electrolytic Solutions. Part II. Measurements." Journal of Chemical Physics 20(7):1077.

Scott, J. H., R. D. Carroll, and D. R. Cunningham. 1967. "Dielectric Constant and Electrical Conductivity Measurements of Moist Rock: A New Leboratory Method." Journal of Geophysical Research 72(20):S101. 


\section{Holographic Impulse Radar for Imaging Buried Waste Containers in Low Conductivity Soil}

H. D. Collins (Automation and Measurement Sciences)

The objective of this work was the preliminary development and evaluation of a multichannel holographic impulse radar front-end for highresolution imaging of (i.e., few meters) buried waste containers, etc., in low conductivity soils. PNL has already developed the holographic impulse imaging software for use with a single channel impulse front-end. A front-end with matched bi-static antenna array will be designed and constructed for this application and preliminary experiments with a 5-foot linear scanner in sandy soil with buried targets will be conducted to verify the imaging resolution capabilities. This work is necessary before proceeding to a full-scale linear array for nearreal-time imaging of subsurface targets.

The objective to develop high-resolution imaging of buried waste using impulse holographic techniques developed at PNL was achieved.
Imaging of buried waste for identification and location is extremely important for its safe retrieval and cleanup. PNL is committed to applying its highresolution holographic technology to this critical problem.

The funds provided for this project produced some very exciting results with shallow buried simulated waste cortainers in dry sand from the Hanford area.

Subsurface targets were imaged at depths greater than 1 meter in the laboratory tank in EDL. The experimental results of this effort were used to win a major DOE program for the design, construction, and evaluation of Real-Time Holographic Array Buried Waste Imaging System for BWID at Idaho National Engineering Laboratory. The system will be used to image shallow buried waste (i.e., less than a meter) during the dig face operation at Idaho National Engineering Laboratory. 


\title{
Machine Learning
}

\author{
R. W. Quadrel (Energy Sciences)
}

The objective of this project was to explore and develop a new competency in machine learning. This technology allows computers to acquire knowledge and expertise automatically from humans by observing their behavior as they solve problems. Observations are collected, organized, and classified incrementally, allowing the computer to continually deepen its knowledge base. In theory, the longer that the computer watches the human expert, the "smarter" it becomes. As the computer is exposed to an increasing number of examples and training cases, it learns how to solve similar problems more efficiently-even problems that it has never encountered before. This technology has the potential for fundamentally changing the way in which advanced computer-based tools are developed and used in a broad range of applications.

Our research consisted of two major tasks: technology evaluation and prototype development. In the technology evaluation task, we reviewed the literature to assess the current state of machine learning research. We focused on the theory and application of supervised inductive learning, one of the most mature techniques used to accomplish automatic learning.

In our second task, we developed and demonstrated a prototype application that uses supervised inductive learning techniques to solve a real-world problem. The prototype, called SmartMail, learns how to classify incoming electronic mail messages according to a user's preferences.

\section{Technology Evaluation}

Our approach was to first define the overall context of machine learning research and to then critically evaluate current research in our selected focus areasupervised inductive learning.
We found two basic methodologies for performing supervised inductive learning: the version spaces approach and the decision tree approach. During our experimentation, we discovered computational limitations to the version spaces approach; consequently we concentrated our efforts on building prototypes that use decision trees. We saved considerable time and effort by using a public-domain software package called IND, distributed by the National Aeronautics and Space Administration Ames Research Center.

Results from the technology evaluation task include a summary of the literature and a critical evaluation of work in our selected focus area.

\section{Prototype Development}

In this task, we demonstrated the machine learning technology by developing software that uses supervised inductive learning methods to teach the computer how to classify incoming mail messages according to a user's preferences. Based on observations of how the user sorts messages into folders, the computer was able to induce general rules for handling mail. These rules allowed the computer to automatically classify new mail when it arrived so that, instead of a simple chronological list of new messages, the user found new messages presorted into appropriate folders, completely eliminating the manual classification effort.

In some cases, we found that the computer, like the human, has difficulty learning when training examples are incomplete or inconsistent. We partially overcame this problem by using the Soundex phonetic compression algorithm, which we believe is a unique solution for this problem.

SmartMail was developed for the Sun SPARCStation platform and is currently being tested by selected members of the Energy Sciences Department. 


\section{Conclusions}

This project was successful in showing how supervised inductive learning could be used for classification tasks. We conclude that this capability is particularly useful for computer-based tools that require the automatic development of knowledge bases, as well as for tools that must acquire knowledge in domains that are ill-defined, difficult to model, or subject to rapid change.

We see many potential applications for this technology, which can be divided into subjective and objective learning. The former approach teaches the computer to learn and respond to a user's preferences. Example applications in this area include adaptable user interfaces and automated climate control systems.
Objective learning is an area in which the computer acquires information that is already shared by others. Example applications include computer-based design tools, automated diagnostics, and advanced manufacturing systems.

The technology evaluation and prototype development performed by the Machine Learning research team in FY 1993 represents broad and successful first steps in the development of this new competency. Machine learning is a basic technical capability that can be applied to advanced computer-based tools in nearly every domain. As such, the technology represents an important opportunity. 


\title{
Nonlinear Constrained Optimization Models with Uncertainty
}

\author{
G. I. Fann (Analytical Sciences)
}

The objective of this project was to construct a top-down model for solving generic constrained nonlinear programming problems with uncertainties in the constraints or the objective function.

\section{Problem and Solution Methodology}

We developed and implemented a method for computing with random or uncertain constraints in optimization problems. The basic idea was to convert probabilistic bounds or uncertainty constraints to fixed bounds. This permits fast solution of the optimization problems using standard software. Our work is motivated by a need to solve a waste minimization model (optimal waste loading [OWL]) developed for Hanford waste vitrification. Our method can also be applied to stochastic optimization problems.

The sources of uncertainty in the Hanford optimal waste loading models are physical property variables, which are bounded by parameters determined from experimental data. The viscosity of vitrified waste logs is an example of such a variable.

Mathematically the problem can be described as follows: Given a confidence level $\alpha$, find $\mathrm{x}$ which minimizes $\operatorname{cost}(x)$

$$
\begin{gathered}
\min _{x} \operatorname{cost}(x) \\
x \in \Omega \\
\operatorname{prob}_{\theta}\{f(x, \theta) \in R\} \geq \alpha
\end{gathered}
$$

where $\operatorname{cost}(x)$ is the cost function (e.g., the number of glass logs). The variable $\mathrm{x}$ is a vector of $\mathrm{n}$ variables that determined the cost function (e.g., components of waste oxides). The function $f$ is a vector valued function of $x$ and $\theta$. The variable $\theta$ is a vector of empirically derived parameters in a given region $R$. $\mathbb{Q}$ is another region of $n$-space that bounds the variables $x$ and is usually convex and determined from other constraints.
The uncertainty in the parameter $\theta$ is the source of randomness in the constraint equation and is usually described by a normal approximation to sampling distribution of the regression coefficients if the region $R$ is determined from experimental data. The mean of this distribution is the least squares estimates, and the covariance can be obtained using regression outputs as the covariance of the estimated coefficients.

We replace

$$
\operatorname{prob}_{\theta}\{f(x, \theta) \in R\} \geq \alpha
$$

by the fixed bound

$$
\begin{gathered}
\text { minpropval }+ \text { uncert } \leq f(x, \theta) \\
f(x, \theta) \leq \text { maxpropval - uncert } \\
\text { uncert }=w\left[x^{s} C x\right]^{5}
\end{gathered}
$$

where minpropval and maxpropval are derived from experimental data. The scalar $w$ is the weighting factor. The matrix $\mathrm{C}$ is the covariance matrix.

\section{Implementation}

We purchased a demonstration version of the optimization package GAMS/MINOS, which permitted us to solve constraint nonlinear programming problems with about 2000 variables. The simple waste loading model was formulated using the modeling language GAMS and solved successfully in under 2 seconds on a SUN Sparc II workstation using the fixed bound constraints. If we were to use the standard method of evaluating the probability constraint, each run would be about a minute. For large scheduling problems where there may be hundreds of runs, this increased speed is significant. 


\title{
Portable Operations and Maintenance Information System (POMIS)
}

\author{
R. F. Szydiowski (Energy Sciences)
}

The objective of this project was to develop an advanced demonstration of the hardware and software necessary to produce a complete portable field-based operations and maintenance tool.

\section{Project Status}

The first POMIS development activity was selection of an appropriate pen-based computer system to support the POMIS objectives. The equipment selection was conducted in cooperation with an extensive pen-based computer evaluation being conducted as part of a separate energy audit development project. The result was savings of 60 manhours, or 15\% of the POMIS project funds. The Energy Sciences Department's interest in POMIS resulted in a commitment of $\$ 7.5 \mathrm{~K}$ to purchase of a pen-based portable computer system from Grid Convertible with Grid PenRight! software.

The set of three Smat Wireless Sensors were developed using Proxim's ProxLink spread spectrum wireless RS232 transceivers and DGH D Series RS232 sensor modules. These commercial components were assembled with a battery pack to demonstrate the functional features of smart wireless sensors. The final smart wireless sensor design would have to be smaller and smarter.

The limited funding for this project did not allow development of the actual operations and maintenance diagnostics or the central data base system. These functions were simulated by the POMIS demonstration software. The PenRight! software development system was selected for its ease of use, but future development will require reevaluation to determine the most appropriate development system.
The primary deliverable of this POMIS development project was a very simple prototype of the POMIS system which can be used to demonstrate the potential of this concept to DOE and others. This was successfully completed.

The initial application is building heating, ventilating, and air conditioning equipment operations and maintenance because there is a growing interest in utility sponsored operations and maintenance incentive programs. However, POMIS capabilities extend to a variety of other field monitoring and operations and maintenance activities, from medical monitoring to production equipment diagnostics, which are not currently possible.

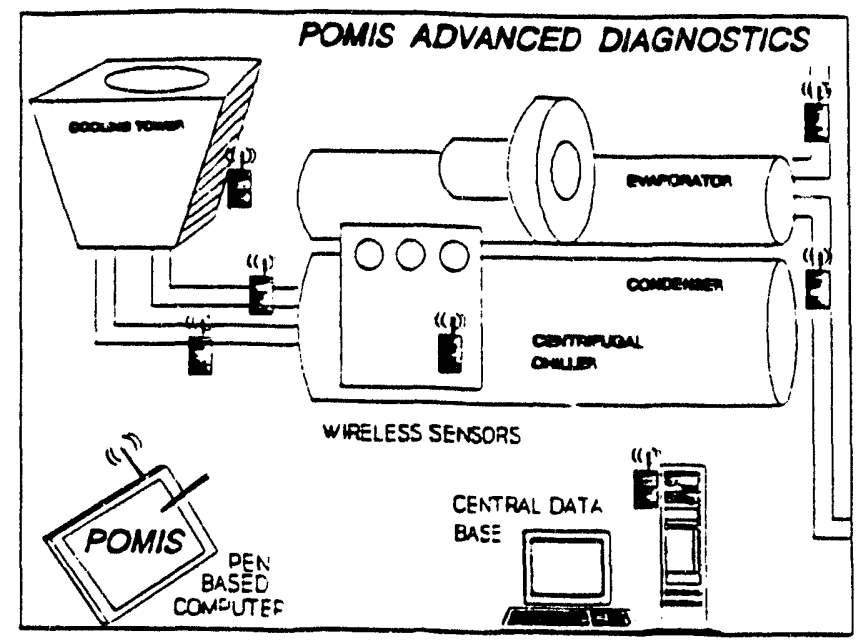

Figure 1. The POMIS Applied to Chiller Operations and Maintenance and Diagnostics 


\title{
Probabilistic Finite Element Analysis
}

\author{
M. A. Khaleel (Automation and Measurement Sciences)
}

A general framework for first- and second-order reliability analysi of structures with geometrical nonlinearity was developed for the analysis of aging structures and facilities. The structure is either linear or nonlinear elastic, and is subjected to static loads. The material properties, geometry, and external loads of the structure are considered as random variables or random fields. The failure criteria of the structure is expressed in terms of limit-state functions.

\section{Probabilistic Finite Element}

Combining the finite element method and the reliability method into a general framework for the reliability assessment of structure is the basic effort in this project. During FY 1993, we purchased from the University of California at Berkeley, the general purpose reliability code, CALREL. The program was tested at PNL. PNL staff members were able to obtain the source code of the finite element code FEAP (Finite Element Analysis Program) from Professor Robert Taylor at the University of California Berkeley at no additional cost. Substantial extensions are made to the Finite Element Analysis Program to incorporate computing the gradient of the response of the structure. The derivatives of the element resisting forces with respect to the basic random variables were formulated on the element level and assembled in the global coordinates using conventional finite element assembly routines. The conventional structure of the finite element progran, FEAP, was not altered. To incorporate the Finite Element Analysis Program code into the CALREL code for a reliability analysis, an interface (i.e., connecting) computer code $Z$ was developed. This code defines the control modes, performs space allocation checks, checks the compatibility of the discretization of random fields and finite element mesh, and exchanges information between both programs. An essential component of the reliability program is the specification of the limit-state (i.e., failure) functions that define the failure criteria and their gradients with respect to the basic variables. In the combined computer code (i.e., CALREL-Z-FEAP), these specifications, which are problem dependent, were implemented so they will be provided by the user in two subroutines.

In FY 1994, the CALREL-Z-FEAP program will be used to conduct analysis on aging structures, such as the Hanford waste tanks.

\section{Presentation}

Khaleel, M. A. "Reliability Analysis of Concrete Girders," to be presented at the Internationdl Conference on Reliability, Quality Control and Risk Assessment, Seattle, Washington, April 1994. 


\title{
Remote Moisture Measurement by Electromagnetic Induction
}

\author{
R. L. Hockey (Automation and Measurement Sciences)
}

The objective of this project was to experimentally investigate an electromagnetic induction technique for moisture measurement in waste tank simulants. Several parameters critical to showing feasibility were explored. This method was evaluated for its potential to provide information unobtainable with existing technology.

The thermal stability of waste tanks containing sodium-nickel-ferrocyanide has been linked to having sufficient water in the waste. Therefore, once a safe range of water concentration has been established, a method of measuring water concentration will be necessary to guarantee tank safety. Moisture measurements must be able to determine the distribution of moisture over large areas in a waste tank.

Electromagnetic induction measurement techniques can provide information about the amount of water absorbed within a substance by measuring its electrical conductivity. Electromagnetic induction methods have been successfully used by geoscientists for measuring the water content of the earth, in and around a borehole. A similar technique was recommended for the single-shell waste tanks in FY 1992. Several questions were raised by tank safety experts regarding the applicability of this tecinnology for use in waste tanks. Their questions centered around how the unknown contents in a waste tank, other than moisture, may affect an electromagnetic measurement.

The experimental apparatus development needed for this work included the design and construction of induction coils that were connected to an impedance analyzer/computer data acquisition system. The coils were inserted into dry sand and the coil impedance was measured as moisture cuntaining dissolved $\mathrm{KCl}$ was added in increments of $5 \%$ by volume. Impedance was measured over the frequency range from $100 \mathrm{~Hz}$ to $1000 \mathrm{KHz}$. Five-percent changes in moisture were clearly identified.

To help answer the question of the effect of metal objects on this type of moisture measurement, small copper strips were inserted into the sand before the ionic water mixture was added. At frequencies below about $80 \mathrm{KHz}$, the same results as without the copper strips were observed, except all impedance values were decreased by the same amount. At frequencies above $80 \mathrm{KHz}$, this impedance shift was not present because the induced electromagnetic field could not penetrate far enough to reach the copper strips. The frequency controlled penetration depth is one of the major advantages of this technique for determining the distribution of moisture. By increasing or decreasing the frequency of current applied to a cnil, smaller or larger investigation depths are expected to occur in a waste tank.

Results from this study answered som $:$ imiportant questions posed in FY 1992, but befcre this method can be developed further, some remaining questions must be answered. Follow-on work will be directed toward a comparison between the electrical properties of core samples taken from single-shell tanks and simulated materials, and the minimum moisture measurement accuracy that could be expected when unknown waste tank contents are monitored under conditions known to exist inside the single-shell tanks.

Other Accomplishments

Presented results at the Tank Instrumentation Assistance Panel and other scientific meetings at Hanford. 


\title{
Sensing Applications of Electrorheological Fluids
}

\author{
J. W. Griffin (Automation and Measurement Sciences)
}

\begin{abstract}
The objective of this project was to investigate acoustic- and optical-based sensing schemes which incorporate electrorheological fluids. Electrorheological fluids are a solid suspension which changes its viscosity upon application of an electric field. The viscosity change is the result of development of an ordered structure in the fluid as particles in the solid suspension orient themselves with the applied electric field. The resultant ordering is accompanied by an increase in shear strength and the introduction of optical anisotropy.
\end{abstract}

The fact that electrorheological (ER) fluids exhibit long-range order under the influence of an electric field can be exploited as a potential sensing mechanism. One scenario would pursue detection of the electrorheological fluid viscosity change induced by an ambient, static electric field. Such a change could be detected as a perturbation in the acoustic impedance of the fluid (e.g., via delay-line oscillator methods). The electric field causes solid particles in the electrorheological suspension to form long chains oriented parallel to the E-vector. These long strands, with dimensions on the order of visible light wavelengths, can potentially be used to induce optical effects in (i.e., to modulate) a traversing optical beam (e.g., due to light polarization/depolarization, diffraction, refraction). This LDRD project was directed toward a study of optical phenomena which could be observed when an electric field was impressed upon a simple electrorheological fluid "wafer."

A simple, chemically-inert electrorheological fluid was made from a suspension of corn starch in vegetable oil. A variety of classical electrorheological fluid effects were photographically documented using this material sandwiched between two aluminum electrodes. For the optical effects experiments, a commercial electrorheological fluid provided by Lord Inc. was sandwiched between two indium-tin-oxide coated fused silica disks. This milky liquid is not optimal for optical applications because of high optical extinction due to scattering (due to the refractive index mismatch between the solid particles and the suspension fluid). A British supplier was identified who manufactured a refractive-index-matched electrorheological fluid (with minimal scattering losses) but since the liquid was an organic carcinogen it was decided not to perform experiments with this material. Thickness of the electrorheological fluid "wafer" (i.e., the spacing between plates) in our sandwich cell was 1 to $2 \mathrm{~mm}$. The indium-tin-oxide coating provided an electrical contact with the fluid yet was transparent enough to allow a HeNe laser probe beam to traverse the fluid volume with minimal attenuation. The major contributor to propagation loss was scattering by the solid particles in the electrorheological fluid. The probe beam propagation direction was parallel to the electric field vector (and likewise parallel to the orientation of the electrorheological fluid "chains" during application of the electric field). No reproducible optical effects (polarization changes, transmission changes, diffraction effects) were observed in the transmitted HeNe beam when a de electric field was applied to the fluid "wafer." This null result was attributed to

- overwhelming optical scattering effects in the electrorheological fluid volume

- insufficient interaction length (1 to $2 \mathrm{~mm}$ ) of the probe beam (the thickness of the electrorheological fluid "wafer" can be increased, but this requires a higher applied voltage to maintain a given electric field strength in the fluid)

- insufficient radiance/polarization stability in the $\mathrm{HeNe}$ laser probe beam (time-dependent variances in the laser output beam possibly dwarfed electricfield-induced effects in the electrorheological fluid).

LDRD project financial resources were exhausted prior to initiation of electrorheological-fluid acoustic impedance studies. It is felt that detection of acousticproperties perturbations in electric-field-stimulated electrorheological fluids still remains a viable sensor mechanism (e.g., for electric fields). 


\section{Sonar Impulse Target Strength Holograph}

H. D. Collins (Automation and Measurement Sciences)

Near-field full-scale vehicle target strength data are extremely difficult and expensive to obtain using conventional far-field techniques. Target strength holography appears to be a rather unique technique that will be investigated in this study for its efficacy to provide acoustic near-field target strength measurements.

The major objective of this project is the simulation of a full-scale sonar impulse target strength holography system using a small-scale model in a water tank at PNL.

The system has been developed using high frequency acoustic transducers scaled with respect to the model.
The system consists of a holographic processor, computer image/target strength algorithm, and $\mathrm{x}-\mathrm{y}$ scanner.

Completion of the system occurred in the last week of September. Preliminary experimental results consist of high resolution holographic images at the higher frequencies.

A unique low frequency bi-static concept using the Battelle self-scanning buoy developed at BMI and Sequim has been developed.

We believe this technique is viable for obtaining extremely low frequency full-scale bi-static target strength measurements in the ocean. 


\section{Spectroscopic Holography}

T. M. Sloane (Automation and Measurement Sciences)

The objective of this project was to demonstrate the feasibility of performing spectroscopic holography in a gaseous medium containing atoms or molecules with an absorption in the visible or infrared region of the spectrum. If successful, this technique would provide three-dimensional spectroscopic imaging of the concentration of an atomic or molecular absorber in an otherwise non-absorbing medium.

We have completed a literature search, and have found two reports of preliminary experiments performed by others which are generally similar but different in potentially significant detail from our current investigation (Rubin and Swain 1991; Trolinger et al. 1992). Near-resonant scattering of light from sodium and the hydroxyl radical has been observed by others, but only light scattered in the forward direction was detected and used to construct a hologram. As a result, only line-of-sight information was obtained. No attempt to detect light scattered in any other direction was reported. In contrast, our approach is aimed at imaging the backward-scattered and/or isotropically-distributed components of the scattering. This will eliminate interference due to the unscattered light and will provide a better opportunity for obtaining three-dimensional information from the hologram.

We have chosen cesium as the target species for our investigation because it will allow us easy access to existing equipment in the laboratory of Dr. Robert Miller in the Materials and Chemical Sciences Center. His laboratory is equipped with a narrow-line $(500 \mathrm{kHz})$, long coherence length, tunable ring laser.
We have performed initial experiments on an effusive cesium beam in a vacuum chamber. A schematic diagram of the apparatus is shown in Figure 1. The light source was an argon ion-pumped titaniumsapphire laser. The laser wavelength corresponding to a resonant absorption was calibrated with two-photon ionization of cesium.

The experimental procedure for attempting to observe near-resonant enhanced scattering of the laser light involved tuning the titanium-sapphire laser through the absorption while observing the reflected radiation with a silicon-based video camera detector and a silicon photovoltaic cell. No near-resonant scattering was observed at $180^{\circ}$ with respect to the laser beam. The camera was also positioned at a $90^{\circ}$ angle with respect to the laser beam to observe sideways-scattered light. A considerable amount of light was detected in this configuration, but it is not clear how much of this light was resonance fluorescence and how much was the desired coherent scattering. The next step will be to determine how much of this light is polarized in the same way as the laser beam. Resonance fluorescence should be either depolarized or should be polarized differently from the laser, whereas the coherent scattering will retain the polarization of the laser. These experiments will be carried out in FY 1994.

\section{References}

Rubin, L. F., and D. M. Swain. 1991. "Near-Resonant Holography." Opt. Lett. 16:526.

Trolinger, J. D., R. K. Hanson, B. Yip, and B. Battles. 1992. "Resonant Holographic Interferometry - a Multipoint, Multiparameter Diagnostics Tool for Hypersonic Flow." NATO Advanced Research Workshop. 


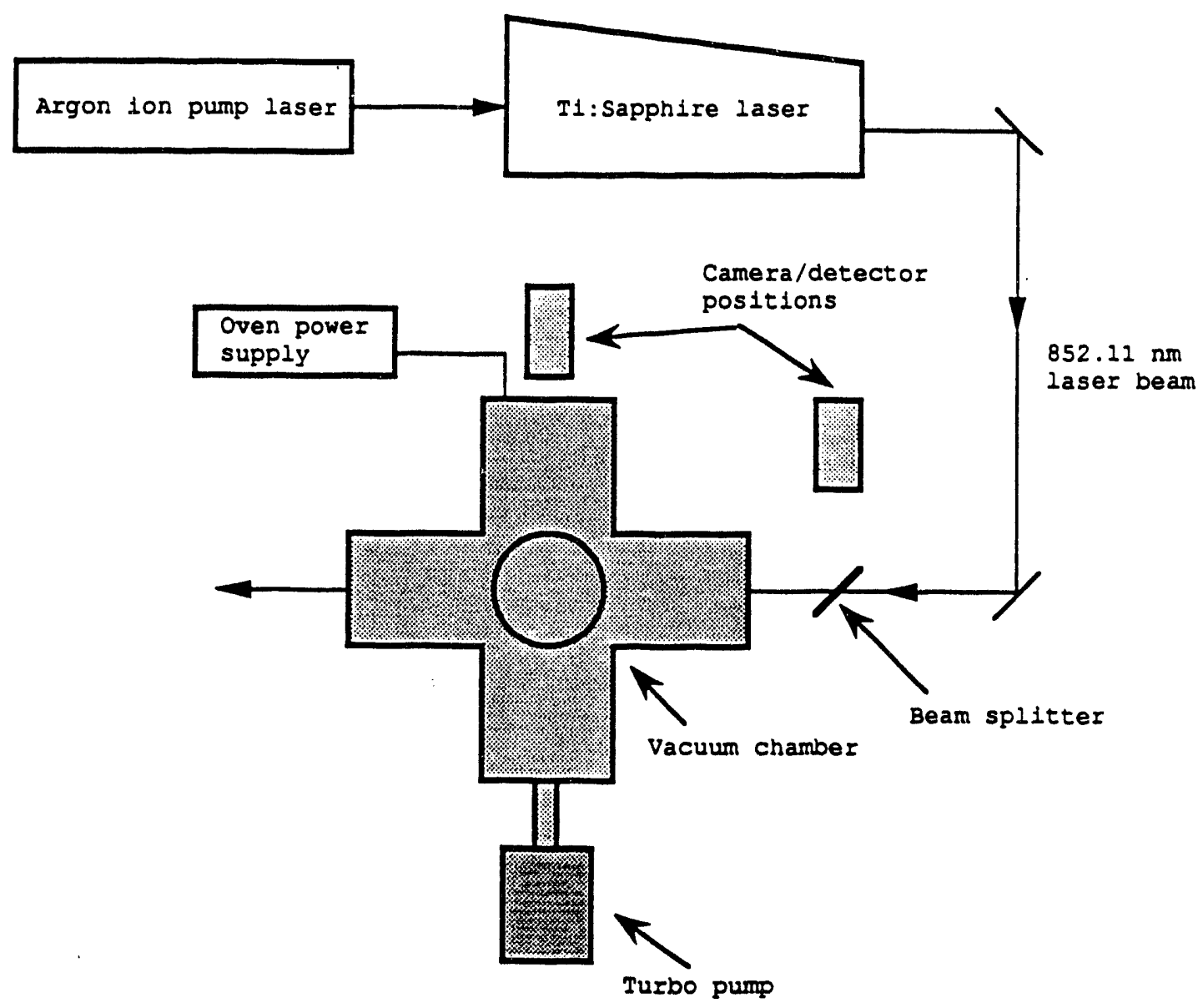

Figure 1. Diagram of Major Equipment 


\title{
Video-Based Imaging Interferometry
}

\author{
C. R. Batishko (Automation snd Measurement Sciences)
}

$A$ variety of video-based imaging interferometric techniques have recently received increased attention in the literature. These techniques make use of coherent light so as to result in interference among multiple video frames or between a stored speckle field video image and a live speckle field video image. In addition, recent publications increasingly report success in making holograms though single mode optical fibers. This project will attempt to develop the imaging interferometry techniques to the level of tools available for routine problem solving, then investigate the capability of implementation through fiber optics for nondestructive testing in inaccessible locations.

One traditional difficulty in applying holographic interferometry to industrial inspection has been the need for recording holograms on high resolution film/plate and the accompanying delays and inconveniences of wet chemical processing. Recent advances in multiframe, phase-stepping video holographic interferometry suggest that the time may be appropriate to develop these methods for use as nondestructive testing tools, at least for routine use in the laboratory. In addition, recent publications report that holography through single mode optical fibers is feasible. The combination of video holography and fiber optic holography would result in a unique, powerful tool for nondestructive testing in inaccessible locations.

This project was aimed at providing a nondestructive testing tool which could be easily set up and used for feasibility studies, demonstrations, and quick response testing. The objectives were to

- complete development and testing of the holographic interferometry system

- develop a user friendly interface for the system to allow it to be used on a routine basis by Electro Optic Systems staff
- investigate the possibility of implementing the technique through single mode fibers.

The intended deliverables included a demonstration of the new capability, a benchtop prototype having a user friendly interface for quick response holographic nondestructive testing or to demonstrate capability, a user's manual and, if possible, a demonstration of imaging interferometry through optical fibers.

\section{Experiment}

During the summer of 1993, the process of debugging the software and refining the optical layout centinued. By late summer, the software modules were debugged, and a successful demonstration was anticipated.

The experimental apparatus shown in Figure 1 consists of a traditional holography configuration with the exceptions that a folding mirror in the reference arm of the interferometer is mounted on a microtranslation stage to provide controllable phase stepping, and the film/plate normally used for recording the hologram is replaced by a video camera. The object under test is a composite plate into the back of which have been milled several recesses including circles and "plus" sign-shaped crosses. One of the crosses was used for the results illustrated in this report.

The process consists of acquiring a set of fourteen consecutive frames at $\mathbf{3 0}$ frames per second of an unstressed sample. These are processed to provide a phase map. The sample is stressed-in this experiment, by heating the sample with a stream of warm air-and a second set of fourteen frames acquired and processed. The two are subtracted and further processed to give a phase difference map of the object, which shows anomalous mechanical deformation due to the stress.

At the time of the :ollow-on FY 1994 LDRD proposal, a real-tine fringe algorithm had been implemented which provides real-time video speckle interferometric imagery to allow the fringe field to be 
monitored at video rates. While not quantitative, or even providing images of flaws in the samples, this mode enables real-time monitoring of fringe motion and distortion which could be the result of flaws, and allows the automatic video holography sequence to be triggered at an appropriate moment.

However, in spite of the utility of this algorithm, results did not correlate well with those reported in the original paper. It was thought that perhaps details of one or more process steps and intermediate images involving phase unwrapping might have been missing from the source paper. This step involves transforming a phase map image having repeated zero to $2 \pi$ cycles, to a phase map image having a continuous range of $-n \pi$ to $+n \pi$.

Several algorithms were tried to accomplish this step, and results confirmed that this was the right direction, as well as a need to reduce the noise in image acquisition and/or interim steps to achieve better discrimination. By the end of the fiscal year, the results were clearly approaching those reported in the original paper, and the development had begun to focus on noise reduction by trying to identify a more optimal spatial filter for smoothing.

\section{Results}

During FY 1993, the measure of success in the experiment became the degree to which the image sequence matched the sequence reported in the original paper.

Figure 2 is a set of images scanned from Vikhagen's original paper and a corresponding set of PNL images. Both have been enhanced for clarity. They represent different flaws in different substrates, so the details of the images are not expected to be comparable. However, they are comparable in the nature of the illustrated result. That is, pairs of images represent the same stage in processing the dataset. Clearly, PNL's final image is not as clear a representation of the flaw in the PNL sample as the Vikhagen result is of its flaw. But the comparison illustrates that results are approaching the published results.

It is particularly interesting to note that the PNL result, while it does not clearly show the flaw (a cross having equal length arms located in the diamond shape in the second PNL image from the bottom) it does show patterns which we believe are associated with the composite layup pattern. The appearance that the bottom arm of the cross is longer than the others may be due to non-uniform thermally-induced mechanical stress due to the presence of another fabricated flaw (a circular cutout) near the bottom edge of the image, directly below the cross.

\section{Conclusions}

The results show that a considerable amount of progress was made during FY 1993. The investigators have good reason to believe that reaching comparable results to those published is primarily a matter of noise reduction. Emphasis on work in FY 1994 will be on noise reduction and implementing user friendly hardware and software.

\section{Reference}

Vikhagen, E. 1990. "Non-Destructive Testing by the Use of TV Holography and Deformation Phase Gradient Calculation." Applied Optics 29(1):137-144. 


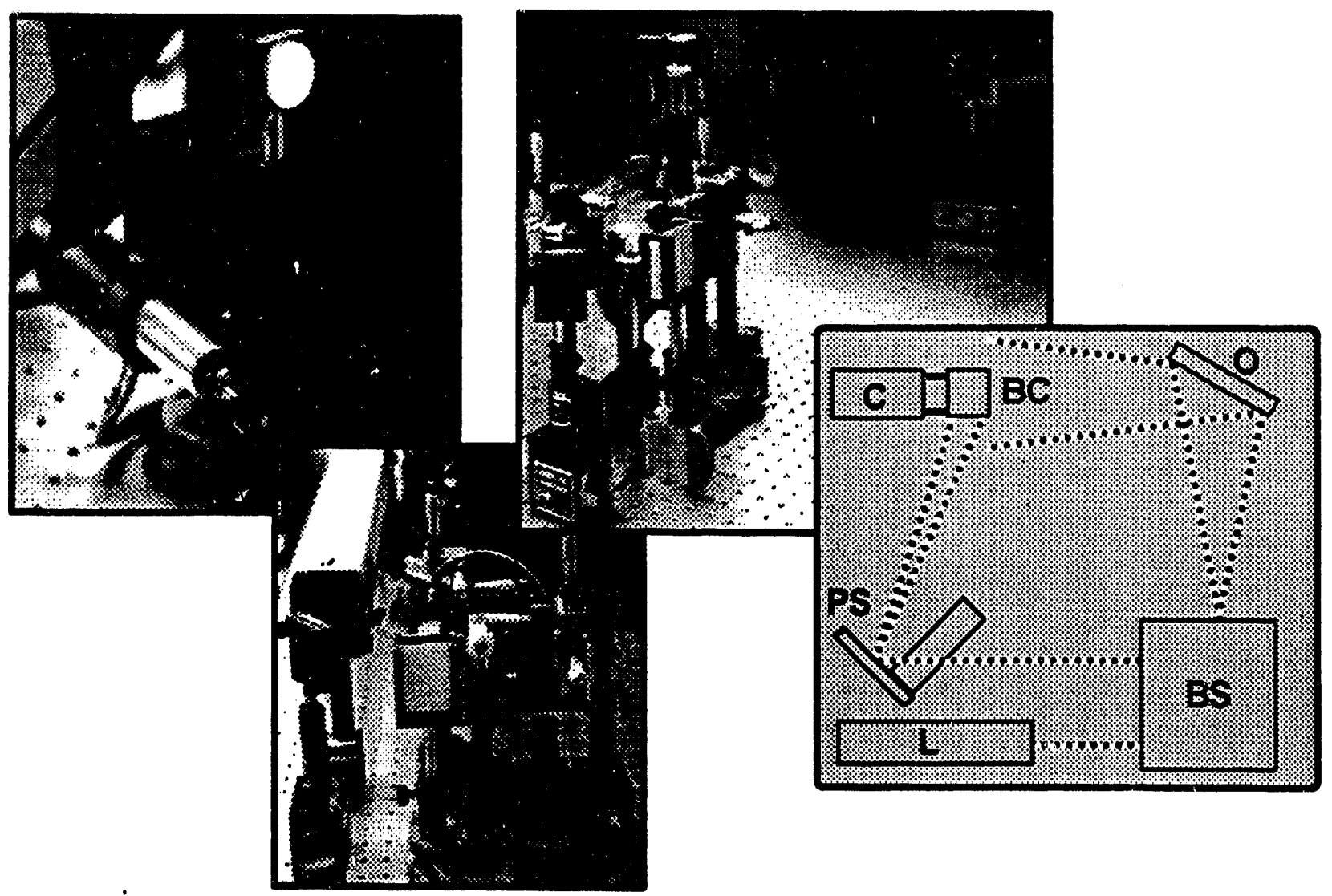

Figure 1. Video Holography Hardware Configuration: laser (L); beamsplitter (BS); object under test (O); phase stepper (PS); beam combiner (BC); camera (C) 


\section{VIKHAGEN}
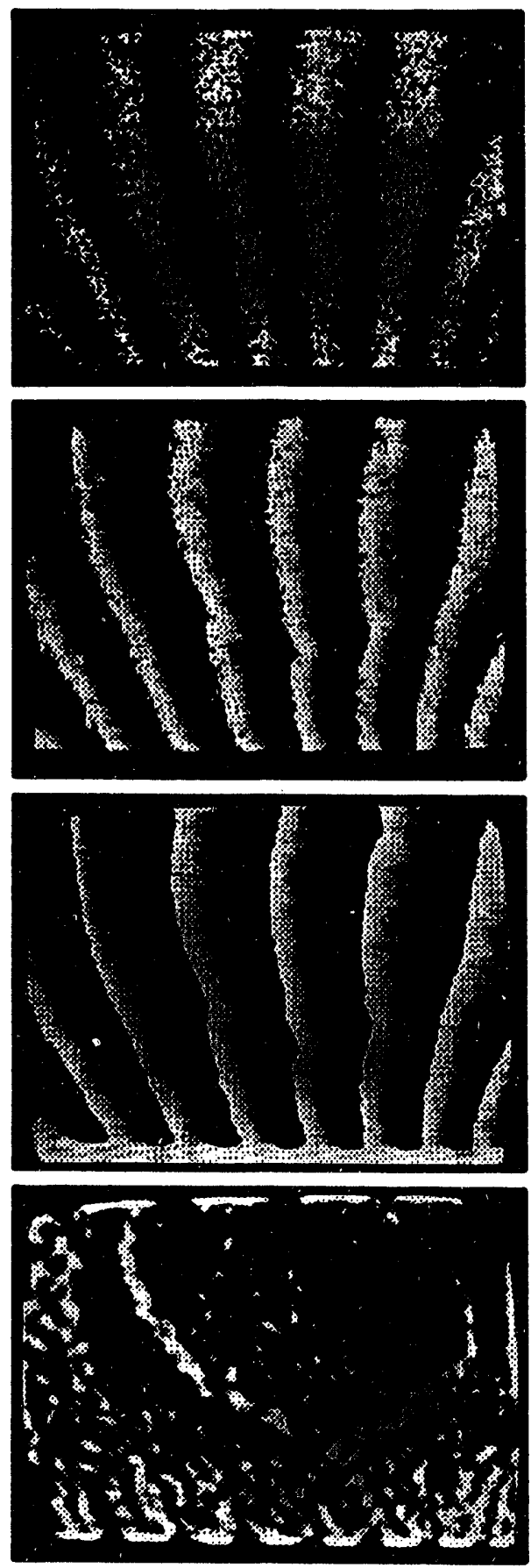

PNL
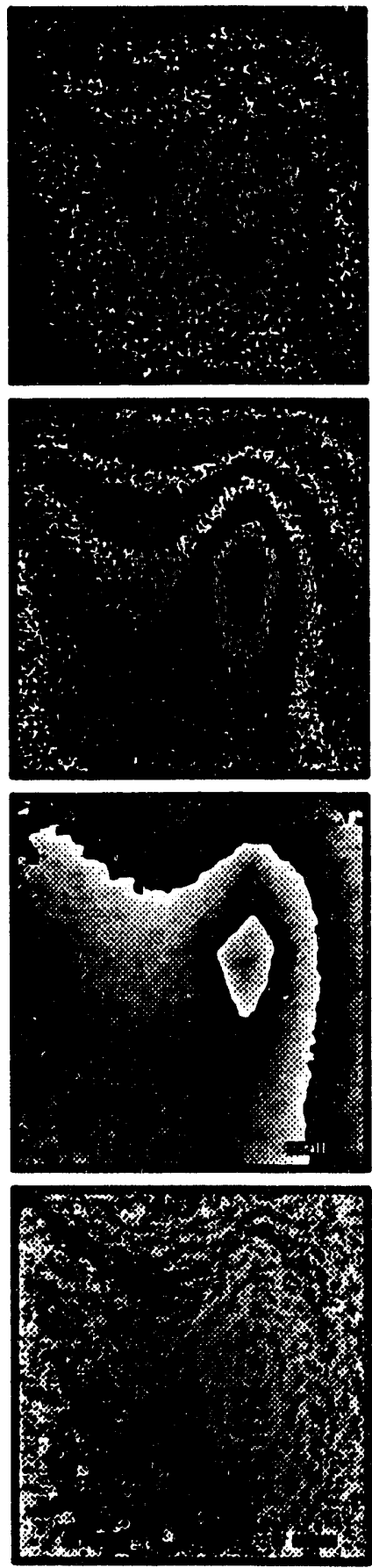

Figure 2. PNL Results Compared to Those Reported in the Source Paper. The two represent different flaws in different substrates, 80 are not intended to be comparable in content. However, they are comparable in terms of the effects of the processing algorithms used to extract a flaw configuration. PNL's results do not clearly discriminate this flaw in this substrate. However, it is interesting to note that the composite substrate layup pattern is detected. All images were enhanced for printing. 


\subsection{Earth and Environmental Sciences}




\section{Advances in Desktop Atmospheric Dispersion Modeling}

K. J. Allwine and X. Bian (Atmospheric Sciences)

The objective of this work was to demonstrate that coarse-grained parallel computer technology can be applied to desktop atmospheric dispersion modeling by

- preparing an easily upgradeable user-interface and output package for the existing singleprocessor version of PGEMS

- determining performance characteristics (e.g., execution speed, accuracy) of the singleprocessor version of PGEMS

- identifying and purchasing a coarse-grained parallel computer

- installing PGEMS on the coarse-grained parallel computer

- demonstrating the potential for scientific improvement in PGEMS using parallelprocessing by incorporating an advanced particle diffusion scheme into PGEMS

- determining performance characteristics of the parallel-processing version of PGEMS and comparing these performance characteristics with the single-processor version of PGEMS.
This project began in the last quarter of FY 1993 during which time Mr. Bian began learning the PGEMS atmospheric dispersion model. This included reviewing the source code and exercising the model over a range of input conditions. The initial work performed by Mr. Bian will allow him to immediately begin development of a new user-interface for PGEMS in FY 1994. Also during FY 1993, various computer vendors were contacted about upgrading personal computers (both IBM and Macintosh) to coarse-grained parallel machines. 


\title{
Aquifer Heterogeneity Identification
}

\author{
C. R. Cole (Geosciences)
}

The goal of this project was to initiate an investigation of a new approach to the very difficult problem of identification of aquifer heterogeneity. important to transport. PNL's current expertise in high resolution data set generation and numerical laboratory testing was augmented through addition of new code capabilities; a three-dimensional particle tracker code and codes that provide an initial approach to parameterization of heterogeneity related to hydrologic system origin and evolution. In addition, other state-of-the-art methods for developing petrophysical models of various distinct geologic facies and images of aquifer systems structure based geologic origin were investigated through literature and field studies in collaboration with the University of Arizona. These limited initial efforts are helping position PNL for carrying out proposed research on aquifer heterogeneity identification.

Multiscale heterogeneity of the geohy'drologic environment is currently recognized as one of the most important factors controlling fluid flow and contaminant transport in the subsurface. It is often a major reason for inaccuracy and uncertainty in the prediction of contaminant migration since it is poorly understood and difficult to characterize. Previous numerical laboratory studies with highly detailed stochastic, synthetic data sets have shown that transport processes are sensitive to a broad range of hydraulic conductivity, porosity, and related geochemical property variations, especially to the extreme values and the tails of these distributions.

The new approach involves the use of flow and transport simulations in highly detailed synthetic porous media systems generated based on knowledge of the geologic processes (formational, diagenetic, and structural) that create these natural systcms. These synthetic natural systems can then be used to investigate the value (ability to reduce prediction uncertainty) of quantitative and qualitative geologic information at various levels of detail (resolution, scale) for site characterization and transport prediction.

$\mathrm{A} \mathrm{C}^{++}$object-oriented three-dimensional particle tracking code was developed. This code allows both Fickian and non-Gaussian particle transport simulations to be performed in PNL's numerical laboratory. This code has been designed to operate in conjunction with PNL's high resolution flow codes.

Codes for creating, displaying, and manipulating numerical aquifer images (e.g., GSLIB, KHOROS), as well as the Wabash numerical aquifer image (a portion of these $\mathbf{7 0 0}$ million data nodes are illustrated in Figure 1), were ported and tested. This software was then available for use on other LDRD projects such as the Integrated Environmental Monitoring initiative and the laboratory-wide LDRD effort on high performance computing.

Nino Aimo (University of Arizona NORCUS appointee) completed a literature survey and performed field studies to examine state-of-the-art methods for developing a Middle Ringold aquifer image as was done for the $W{ }^{-}$iash sediments. As part of this effort, various spatial scales of heterogeneity were mapped, a limited number of air permeability measurements were made, and matrix samples were taken at the Middle Ringold outcrop across the Columbia River from Hanford.

Particle transport simulations using the new particle tracker were performed on multigrid flow solutions for portions of the Wabash aquifer image in order to investigate an approach for upscaling dispersivity. 


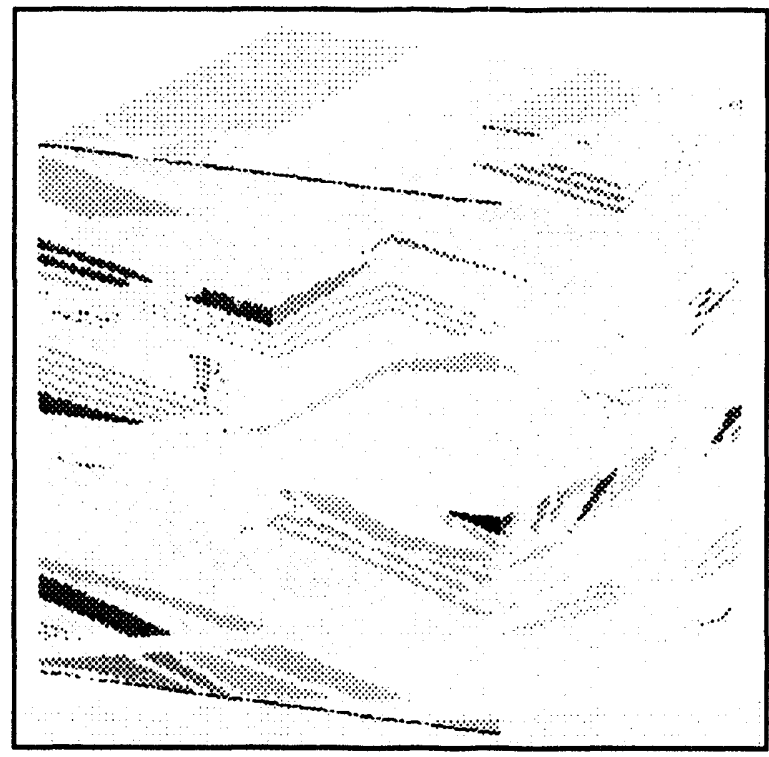

Figure 1. Cutaway View of a Portion of the Wabash River Numerical Aquifer Image (a three-dimensional, highly detailed description of point bar sediments similar to those observed in the Wabash River, USA)

\section{Publication}

A journal manuscript entitled, "Non-Gaussian Particle Tracking: Application to Scaling of Transport Processes in Heterogeneous Porous Media," was prepared and submitted to Water Resources Research on July 28, 1993 (PNL-SA-22067).

\section{Presentations}

Two presentations were made on based on the results of this effort (spring 1993 American Geophysical Union meeting in San Francisco and a Geological Society of America Meeting in Boston, Fall 1993). 


\title{
Bonding and Structural Features Controlling Reactivity on Environmental Surfaces
}

\author{
J. M. Zachara and C. C. Ainsworth (Geosciences)
}

The objective of this project was to probe selected surface chemical reactions to evaluate bonding and structural features controlling reactivity on environmental surfaces using fluorescence spectroscopy. Unique scientific capabilities in the interfacial processes group will be integrated with recently acquired instrumentation in the Molecular Science Research Center to establish a unique state-of-the-art capability to integrate reactions with emphasis on environmental and geochemical interfaces.

This 1-year project was designed to investigate spectroscopic techniques to interrogate the chemical speciation of metals and metal-organic complexes at metal oxide surface/water interfaces. The ability to link spectroscopic evidence of surface species to hypothesized surface chemical reactions and bonding mechanisms at the water/solid interface will lead to a more complete understanding of important geochemical surface reactions that control the fate of groundwater contaminants.

The fluorescence spectra of the $\mathrm{Eu}^{3+}$ cation and the EuEDTA complex was studied in aqueous solution, and in suspension with $\gamma-\mathrm{Al}_{2} \mathrm{O}_{3}$ where appreciable adsorption by surface coordination was known to occur. The use of a SPEX 1680 single photon counting fluorescence spectrometer, and laser photon source in the MSRC permitted spectra to be collected at Eu concentrations on the surface of $\gamma-\mathrm{Al}_{2} \mathrm{O}_{3}$ at less than $10 \%$ monolayer coverage. The fluorescence of aqueous Eu solutions, excited at $393.5 \mathrm{~nm}$, exhibited a broad emission spectra with a primary maximum emission centered at $615 \mathrm{~nm}$. The adsorption of Eu at the $\gamma-\mathrm{Al}_{2} \mathrm{O}_{3}$ /water interface increases from zero to $100 \%$ as the suspension pH increases from 4 to 9 . Conversely, the adsorption of EuEDTA was 4 times less than Eu (25\%) and decreased as $\mathrm{pH}$ increased. The maximum adsorption of EuEDTA was observed at $\mathrm{pH} 5$ and decreased to zero at $\mathrm{pH} 8$. The behavior of Eu and EuEDTA is similar to that observed in studies with Co(II)/Co(II)EDTA, and Co(III)EDTA under similar conditions. Aqueous Eu could be detected and quantified at concentrations as low as $1.0 \times 10^{-6} \mathrm{~mol} / \mathrm{L}$. However, aqueous EuEDTA could be quantified at concentrations orders of magnitude below that of aqueous $\mathrm{Eu}$ alone. This increased sensitivity of Eu in the presence of EDTA is suspected to result from the dehydration of the $\mathrm{Eu}$ ion when complexed with EDTA. The $\mathrm{Eu}^{3+}$ cation sorbed to the surface of $\gamma-\mathrm{Al}_{2} \mathrm{O}_{3}$ exhibited a similar, albeit smaller, increase in sensitivity. 'This latter observation would suggest that Eu sorbed at the $\gamma$ $\mathrm{Al}_{2} \mathrm{O}_{3}$ /water interface partially dehydrates, forming an inner-sphere complex with the $\gamma-\mathrm{Al}_{2} \mathrm{O}_{3}$ surface hydroxyl sites. The EuEDTA $/ \gamma-\mathrm{Al}_{2} \mathrm{O}_{3}$ surface complex, however, exhibits behavior suggesting very weakly associated outer-sphere complexation with the $\gamma-\mathrm{Al}_{2} \mathrm{O}_{3}$ surface.

We have concluded that this technique has great promise and sensitivity to probe metal speciation and bonding at the solid liquid interface. While limited to a strong flourophore such as Eu, it can, by chemical analogy, provide important insights on the interfacial geochemistry of environmentally relevant metal contaminants and their complexes with organic ligands. 


\title{
Coplanar PBC Congener Separation
}

\author{
E. A. Crecelius and L. F. Lefkovitz (Marine Sciences Laboratory)
}

The objectives of this project were to demonstrate that the polychlorinated biphenyls coplanar congeners can be sufficiently isolated from other normally interfering chlorinated substances so as to allow their analysis by gas chromatograph/ electron capture detectors (GC/ECD), and demonstrate this analysis in sediment and tissue matrices.

Polychlorinated biphenyls (PCBs) are considered an ubiquitous environmental contaminant. These compounds tend to bioaccumulate in wildlife, especially fish and the waterfowl that feed upon them. PCB concentrations below $1 \mathrm{ppb}$ can create a health hazard. PCBs are actually a mixture of $\mathbf{2 0 6}$ different compounds. A limited number of these compounds have a chemical and physical structure similar to that of dioxins, which make them significantly more toxic to organisms. PCB congeners with this "coplanar" structure include PCB congeners 77, 126, and 169. Standard analyses for PCBs cannot separate these three compounds from other PCB congeners due to chromatographic limitations. This study attempted to isolate these compounds using a high performance liquid chromatographic separation technique. Two different columns were evaluated, a pyrene column and a fullerene column. Separation was achieved on both columns; however, the pyrene column appeared to provide better separation. Confirmation and quantitation of the coplanar PCBs were performed using capillary colımn gas chromatograph/electron capture detector (GC/ECD).

A mixture of a subset of PCB congeners, including the coplanar congeners PCB 77, 126, and 169, were run through both columns to determine their chromatographic potential for separating the congeners of interest. The fullerene column exhibited coelution of
PCB 77, a coplanar congener, with PCB 105. An injection of PCB 77, 126, and 169 combined was also run. These congeners of interest displayed minimal separation on the fullerene column. Compa ison of the fullerene column to the pyrene column clearly displayed that the pyrene column provides better separation, superior reproducibility, a lower degree of coelution among the congeners, and finer resolution of peak shape. Consequently, further work was focused on the pyrene column.

A total of 31 PCB congeners, chlorinated pesticides, including the DDT series, and PCB aroclor mixtures were run to determine if the pyrene column would also separate them from the coplanar PCBs. Extracts of standard reference materials obtained from the National Institute of Standards and Technology (NIST), including a mussel tissue (Standards Reference Materials 1974), were injected independently and were also part of the determination. These additional materials assisted in determining the potential of other non-PCB compounds to coelute with the coplanar PCB congeners of interest. Complete separation of these compounds from the coplanar congeners was achieved based on the results of the high-pressure liquid chrozaatograph ultraviolet detector. Gas chromatographic confirmation is pending.

Upon completion of data collection, attempts were made at using altarnate solvents for the mobile phase on the pyrene column. A number of different solvents were used and the chromatographic characteristics of each were evaluated.

\section{Other Accomplishments}

We are in the process of summarizing these results for submittal to a peer reviewed publication. 


\title{
Developing a Framework for an Advanced Air Toxics Model
}

\author{
L. A. Mahoney (Atmospheric Sciences)
}

Preliminary results from a 1992 LDRD project have shown that an interactive system can readily be constructed to guide laymen through the logic necessary to choose between a number of dispersion models, each appropriate for a specific application. The goal of this project was to extend this work to not only help select an appropriate model, but to run it. Such a tool would be valuable to EPA and other agency policymakers and would be especially useful in scenario analyses for most of the 190 hazardous air pollutants listed in the 1990 Clean Air Act Amendments. In addition, we hope to perform feasibility studies (though not demonstrations) for applying a similar approach to three separate disciplines in atmospheric science.
The first task accomplished during FY 1993 was a review of the competing software from other sources. The review revealed that the system that had been prototyped during the 1992 LDRD was not significantly ahead of the state of the art as had been believed. It was decided to close out this project and work was discontinued. 


\title{
Extraction of Organic Contaminants by Supercritical Fluid Extraction
}

\author{
E. A. Crecelius (Marine Sciences Laboratory)
}

The objective of this project was to develop sample extraction methods that are fast (reduce labor), use no flammable solvents (safer and cheaper), and generate no hazardous waste disposal problems. These new extraction methods will be used for DOE programs including Hanford Site characterization, other site characterizations, and environmental analysis.

Supercritical fluid extraction has become increasingly popular as a means of removing nonpolar organics such as polynuclear aromatic hydrocarbons (PAHs) from solid matrices for subsequent analytical analyses. Studies have shown that supercritical fluid extraction can achieve comparable results to more conventional extraction techniques in a much shorter time using significantly less organic solvents. After analysis of some relatively complex environmental matrices, however, we have found that the technique is not very user friendly and that currently available methods may not be applicable to analysis of fairly contaminated sediments or tissue matrices.

Samples of both tissue and sediment standard reference material obtained from NIST were analyzed by supercritical fluid extraction. Supercritical fluid extraction analyses were conducted onsite at one of the supercritical fluid extraction manufacturers operations by trained experts in the methodology. In addition, various solvent systems were used during to attempt to both maximize extraction efficiency and to eliminate extraction of unwanted interfering organics.

Chemical analyses included gas chromatography/mass spectrometry for PAH compounds and GC/ECD analyses for PCBs. Sample extracts were not subjected to additional cleanup steps after supercritical fluid extraction to see if the extracts would be clean enough to analyze directly. PAH results appeared to be reproducible between replicates using the same solvent system, however, absolute recoveries were low for all tests. This may have been due in part to technical problems the analysts had with the operation of the instrument. However, the matrices themselves were fairly complex and included contaminated marine sediments and marine mussel tissue. Some solvent systems appeared somewhat more effective than others in the extraction and the systems that worked best for tissues did not necessarily work best for sediments.

PCB quantitation was not possible on the extracts obtained by supercritical fluid extraction due to matrix interferences that were co-extracted. GC/ECD methodology is not as selective as gas chromatography/ mass spectrometry and is subject to interferences. An additional cleanup step would be necessary to analyze the extracts to attempt to remove some of these interferences. Since we were trying to avoid using additional organic solvents, this step would defeat some of the purpose of using supercritical fluid extraction, since any cleanup step would require the use of a significant amount of solvent.

From these tests we have concluded that currently available methods may not be applicable to analysis of very contaminated sediments or tissue matrices, especially for GC/ECD analyses of chlorinated organic compounds. Supercritical fluid extraction instrumentation is still in the developmental stage and routine use of this technique is limit $d$. 


\title{
Modeling of In Situ Biorestoration of Organic Compounds in Groundwater Using High Performance Computers
}

\author{
K. R. Roberson (Geosciences)
}

The goal of this project was to integrate improved biokinetics into a three-dimensional simulator and run it on a massively parallel computer architecture. This is joint work with Mary Wheeler and her group at Rice University.

In recent years, in situ biorestoration has emerged as a promising technology for the treatment of contaminated groundwater. Numerous field and laboratory studies have shown that indigenous microorganisms can degrade harmful compounds in aquifers under both aerobic and anaerobic conditions. In situ biorestoration involves the enhancement of these natural processes by introducing nutrients (e.g., oxygen, etc.) into the subsurface. Future processes may even involve cultured or genetically tailored organisms that would need to be placed in contact with the contaminant. Bioremediation can also be applied in the context of pump and treat methods where the produced water is passed through chambers containing the organisms at the surface, although current cost estimates indicate that this approach does not scale very favorably to large cleanup programs. The emphasis in this project, therefore, is on in situ methods.

The governing equations describing bioremediation processes are coupled nonlinear partial differential equations involving flow, transport, biodegradation, sorption of substrates, distribution of nutrients, and dissolved oxygen, etc. The movement of the organisms in response to chemical gradients may need to be included, as well as redistribution due to flow. Specific models for biodegradation and organism behavior are the subject of current research at PNL and elsewhere. The model used herein represents the reactions as a system of ordinary differential equations which is solved by integration in time. The numerical method includes biodegradation terms as generalized sources and sinks in the transport equations. Monod and Michaelis-Menten kinetics have been modeled successfully, and sorption is treated as a linear equilibrium isotherm.

\section{Technical Progress/Status}

An operator splitting procedure was used to split the physics of flow, transport, and biodegradation. The reactions take place on the shortest time scale, transport next, and flow the slowest evolving. Our simulator used a lowest-order Raviart-Thomas mixed finite element space for the flow calculations using a non-overlapping domain decomposition to introduce parallelism. Each subdomain is padded by "PAD" elements on internal faces. The interface between domains was solved with preconditioned conjugate gradient with Jacobi or balancing preconditioning. Velocities from the flow solution were post-processed to obtain a fully continuous field. The modified method of characteristics was used for the transport part of the solution, so the next step was to use the continuous velocity field and find the trace-back points and evaluate the trace-back integrals. If the trace goes outside of the padded region, the time step is cut and flow recomputed. The final step is setting up and solving the linear system, with the biokinetics acting as generalized sources and sinks.

\section{Conclusions}

Following are some conclusions from this work:

- The characteristic procedure requires no CFL constraint, i.e., it can take very large time steps.

- The procedure is locally conservative in theory and almost in practice (i.e., no significant material balance errors).

- Bioremediation computations scale linearly in parallel.

- Transport and bioremediation scales almost linearly.

- Operator splitting helps load balance the flow, transport, and biokinetics computer effort. 


\section{Application}

We used our simulator on a problem related to the design that will be used for the field bioremediation demonstration that is part of the Volatile Organic Carbon-Arid Integrated Demonstration. This well is fairly complicated with flow entering at the bottom and exiting at the top. The almost-linear scaling means that this method could be used on practical design problems incorporating high spatial resolution in the near future. The opportunity to use this work on a real project is the fruit of success for this LDRD project.

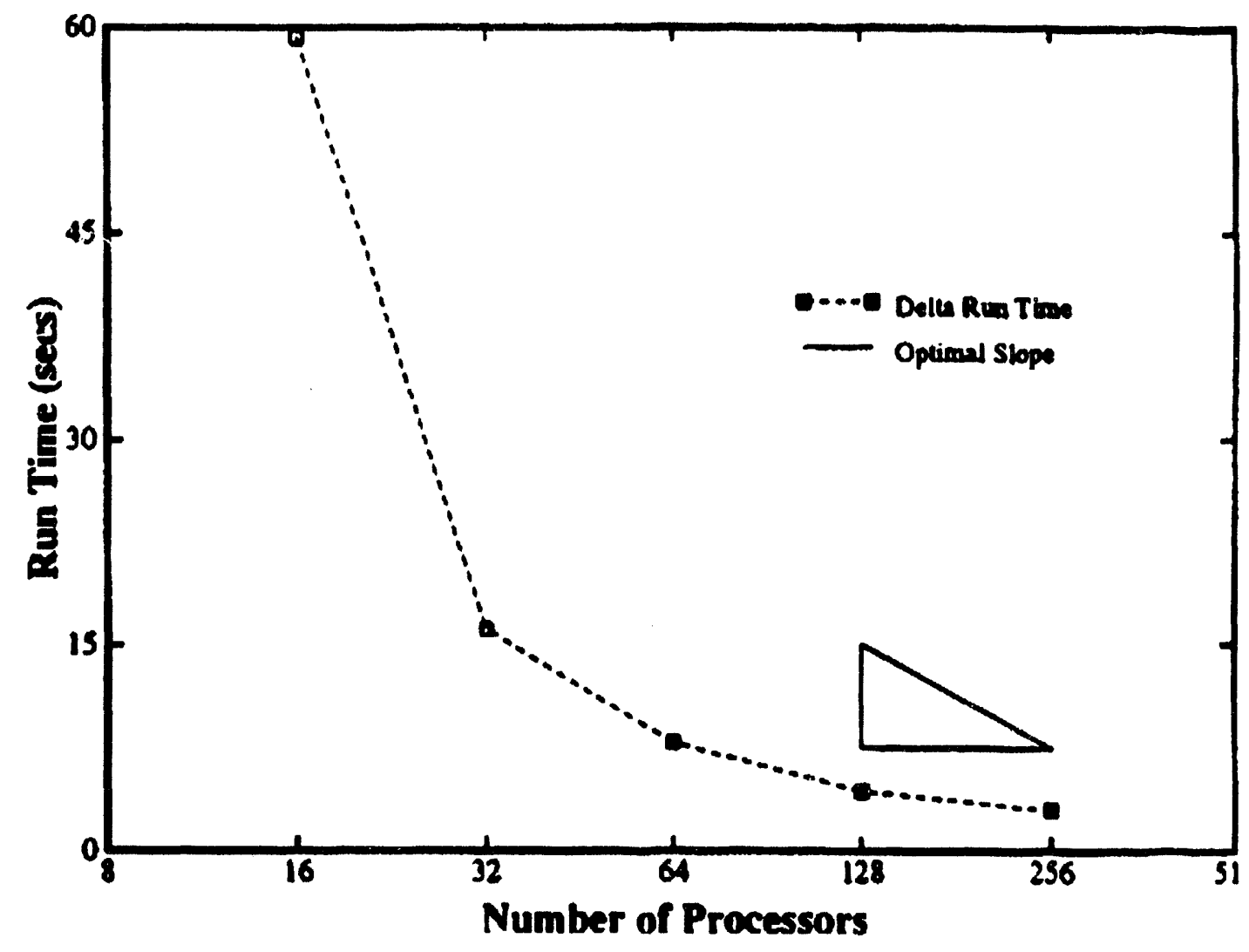

Figure 1. Run Time of the Test Bioremediation Simulation as a Function of the Number of Processors Used 


\title{
Software Development for Analyses of a New Global Meteorological Data Set
}

\author{
D. L. Elliott (Atmospheric Sciences)
}

A recently released global meteorological data set provides new opportunities for potential applications in renewable energy assessment, conventional climatic analyses, and global climate change research. This data set, called DATSAV2 Surface Climatic Database, was developed by the $D O D$ and recently released to the U.S. National Climatic Data Center (NCDC). It is composed of hourly surface weather observations collected and stored from sources such as the Global Telecommunications System. There are about 20,000 stations in the data set and the period of record is primarily 1973 to 1992 . For many countries of the world, the data set contains many times the amount of digital data than could be obtained for sources in the country. For this reason, a much more comprehensive analysis of the meteorological data can be produced using this data set than would be possible otherwise. In this project, a global meteorological data analysis system is being developed to exploit the unique capabilities offered by this data set.

The global meteorological data analysis system (GMDAS) is a comprehensive computer software package developed on the SUN/UNIX workstations running the Xwindows system. The global meteorological data analysis system is designed to extract and statistically analyze meteorological data from the DATSAV2 Surface Climatic Dataset. The output of the statistical analysis is graphical plots using the CADISSPLA software. When fully developed, GMDAS will be an effective tool used to enhance research applications, such as renewable energy assessments and global climate modeling.

Advancements to GMDAS during FY 1993 included the development of wind data statistical analyses products from the DATSAV2 dataset, and DISSPLAbased graphics with appropriate scales for each of the products. In addition, a graphical-user interface menu on the Xwindows system was developed to give the user the ability to produce the graphical analyses desired. A sample of this user-friendly form is presented in Figure 1.

A user inputs the file containing the data extracted from DATSAV2, and is given a list of the meteorological stations for which data is available. The user is then given a choice of which particular plot(s) to produce, and which period of record to use. The user can also have the option to adjust the scales on any particular plot(s).

Future development of the GMDAS can include additional modules to extract various types of data from the DATSAV2 dataset and options to further increase the flexibility of the user interface. 


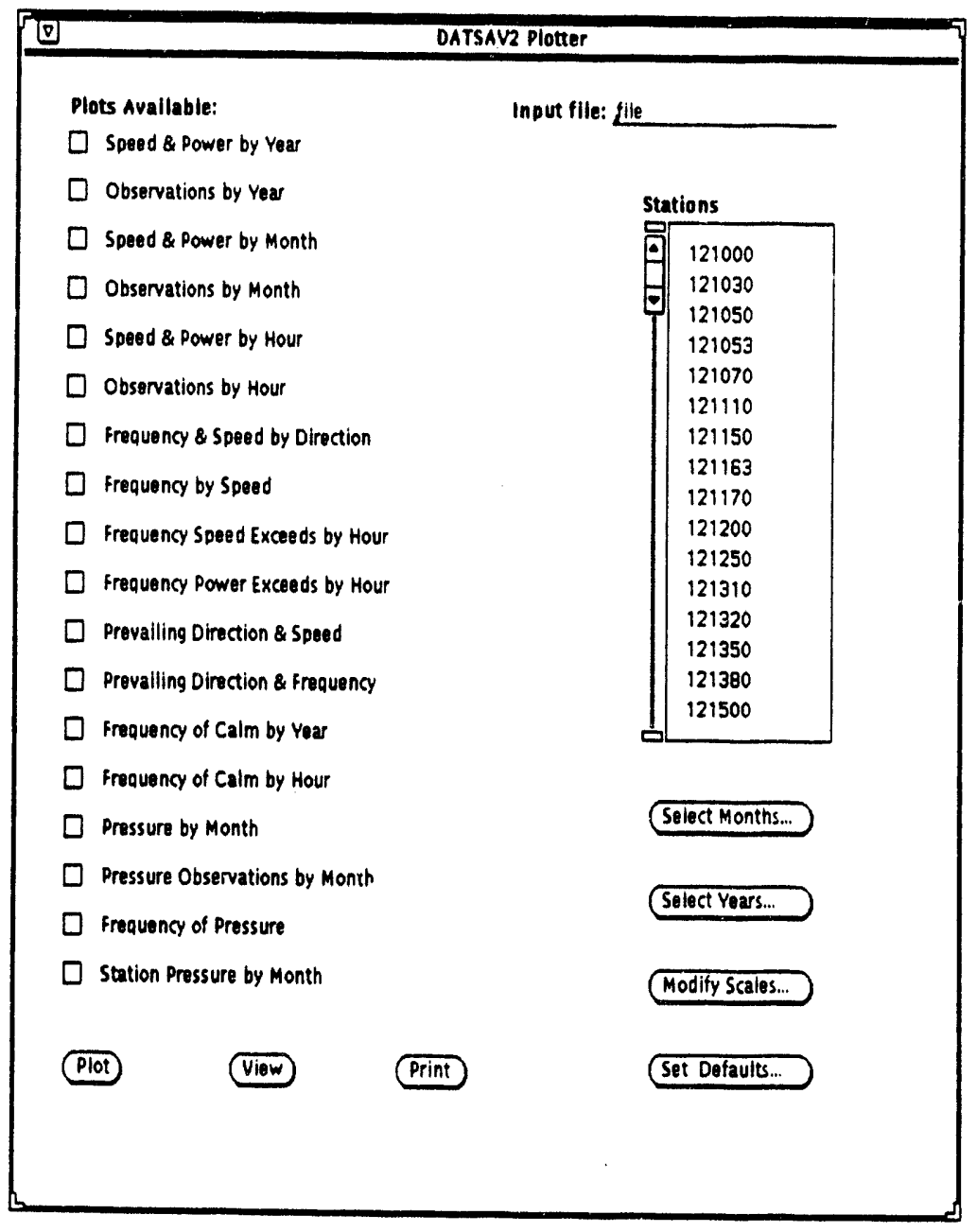

Figure 1. Main Menu for DATSAV2 User Interface 


\subsection{Life Sciences}




\title{
Appliance Use and Cancer
}

\author{
R. H. Lovely (Life Sciences)
}

The objective of this project was to assess whether there are statistically significant associations between extremely low frequency-related cancers and reported use of select household appliances that are known to generate intense magnetic fields. The appliances are used very closely to the body.

The hypothesis that use of personal electric appliances may be associated with increased risk of acute nonlymphocytic leukemia in adults was tested using interview data from a previously completed case control study of 114 cases and 133 controls. Cases were obtained from a population-based cancer registry in western Washington and controls were obtained from the same area by random digit dialing.

Of 32 electrical home appliances on which use data were available for adult acute non-lymphocytic leukemia cases and controls, three motor-driven personal appliances (electric razors, hair dryers, and massage units) were selected a priori because their use represents exposure to higher peak magnetic fields than that from most other home appliances. When compared on an "ever used" versus "never used" basis, use of these appliances collectively was not associated with increased risk of leukemia in the population studies (OR $=0.7 ; 95 \%$ CL: 0.41-1.24).
When the appliances were considered individually, massage units were more likely to have been used by cases than by controls (OR- 3.0; 95\% CL: 1.43-6.32), while hair dryers were more likely to have been used by controls than cases (OR $=0.38$; $95 \%$ CL: 0.22 0.66 ). There was a non-significant tendency for razor use to differentiate the cases from control (OR = 1.33; 95\% Cl: 0.8-2.23). When reported daily time of use was stratified, there was no overall increased risk with increased time of use except for razors $(p<0.05)$. In addition to the analysis of appliance use data from the case control study, we obtained several models of these motor-driven personal appliances and characterized the magnetic fields they produce. Magnetic field flux density and spectral measurements showed that partial body exposure from such appliances may exceed $0.5 \mathrm{mT}$ (rms) at rates-ofchange exceeding $10 \mathrm{~T} / \mathrm{sec}$.

These epidemiological data must be interpreted cautiously because the number of cases is limited and because of proxy reporting of appliance use for deceased cases. Nevertheless, we believe these data indicate that peak magnetic field exposure from personal appliances warrants further investigation as a possible risk factor for acute non-lymphocytic leukemia in adults. 


\title{
Assessment of Hazardous Waste Exposure and Effects
}

\author{
D. D. Mahlum (Biology and Chemistry)
}

\begin{abstract}
Hazardous waste sites contain multiple components, including radionuclides at DOE sites. These complex mixtures pose an immense challenge for the required characterization of potential health effects for waste sites. There is a lack of exposure and dose-effect information for most hazardous waste sites (including those associated with the DOE complex).
\end{abstract}

\section{Major Histocompatibility Complex}

Two experiments were performed with lymphocytes from the owl monkey to determine if the level of a number of cell surface antigens (major histocompatibility complex associated marker) would be affected by exposure to ionizing radiation. In the first study, blood was obtained from six owl monkeys and treated with $0.5,1$, or $5 \mathrm{~Gy}$ of ${ }^{60} \mathrm{Co}$ radiation. Lymphocytes were then isolated and tested with fluorescent monoclonal antibodies to seven different cell surface antigens. The cells were then examined using cytofluorimetry. Two antigens appeared to be responsive, Leu $3 \mathrm{a} / 3 \mathrm{~b}$ and CD45RO. The level of CD45RO-reacting antigen showed the largest change, a decrease of approximately $60 \%$ in the blood that received $5 \mathrm{~Gy}$. Moreover, the decrease appeared to be dose dependent.

The second experiment involved isolating the lymphocytes from whole blood, irradiating them with $0,0.5$, 1 , or $5 \mathrm{~Gy}$ of ${ }^{60} \mathrm{Co}$. Aliquots of the lymphocytes were then incubated with either concanavalin A or phytohemagglutin for $\mathbf{4 8}$ hours to stimulate cell division. The cells were then treated with one of the fluorescent-labeled antibodies as before, and then analyzed using flow cytofluorimetry. Unfortunately, this experiment was limited by the number of lymphocytes available from each animal and by poor performance in culture for those exposed to either the 1 or $5 \mathrm{~Gy}$ dose. However, preliminary data suggest that the binding of both Leu 3a/3b and CD45RO antibodies was decreased by radiation, particularly when incubated with concanavalin A. Despite the limitation of these two experiments, the data suggest that changes in major histocompatibility complex cell surface antigens may serve as markers for radiation damage. Additional studies are needed to confirm these results. However, replication of these preliminary data would suggest that the system could be useful in identifying markers of exposure to radiation. It would then be useful to extend this approach to chemical exposures.

\section{Tumor Transplant Model}

In addition to the search for molecular markers of exposure, we examined the large granular lymphocytic leukemia tumor transplant model (being used in our laboratory for other studies) for its utility in studying the effects of whole animal exposures to radiation and to solvents. In this study, groups of Fischer rats were acutely exposed to $5 \mathrm{~Gy}$ of ${ }^{60} \mathrm{Co}$ radiation, $1500 \mathrm{mg} / \mathrm{kg}$ of CCl4, $1500 \mathrm{mg} / \mathrm{kg}$ of trichloroethylene, $3000 \mathrm{mg} / \mathrm{kg}$ of benzene, or $50 \mathrm{mg} / \mathrm{kg}$ of cyclophosphamide. Four days later, the animals were injected with $5 \times 10^{6}$ spleen cells. Other groups were injected with the same dose of spleen cells and then treated with $\mathrm{CCl}_{4}(500 \mathrm{mg} / \mathrm{kg})$, benzene $(1000 \mathrm{mg} / \mathrm{kg})$, trichloroethylene $(500 \mathrm{mg} / \mathrm{kg})$, or $\mathrm{CCl}_{4}+$ trichloroethylene $(250 \mathrm{mg} / \mathrm{kg}$ of each) five times weekly. Animals were weighed weekly and the spleens palpated regularly for enlargement. In addition, at 7 weeks post-treatment and weekly thereafter, the animals were bled and the packed cell volume determined.

A number of interesting preliminary observations were made during this experiment. First, the animals treated chronically with $\mathrm{CCl}_{4}$ or $\mathrm{CCl}_{4}+$ trichloroethylene failed to gain weight after the first week of treatment. Even when the dosage of the solvents were reduced to half after 5 weeks of treatment, weight did not increase significantly. Secondly, animals receiving the combined solvent treatment died beginning at about 5 weeks of treatment and by 7 weeks more than half had died. Those treated with the larger dose of $\mathrm{CCl}_{4}$ or trichloroethylene alone did not exhibit marked mortality. Third, the animals that received the combined treatment had enlarged spleens that could be detected at 5 weeks after treatment. Other groups, including the irradiated group, did not have detectably enlarged spleens until at least 1 to 2 weeks later. 
Fourth, the animals that received benzene chronically have not presented with enlarged spleen even after 8 weeks. This last result is similar to a previous experiment in which animals treated with benzene did not show symptoms of the disease even 3 to 4 months after injection of the tumor cells. It should be noted that the animals treated with an acute dose of benzene appear to develop the disease at the same rate as vehicle-treated ones.
These results demonstrate 1) the potential use of this tumor transplant system for studying the interaction of toxic materials such as halogenated solvents, 2) the potentiation of $\mathrm{CCl}_{4}$ toxicity by trichloroethylene, and 3 ) prevention of tumor development by administration of benzene. 


\title{
Cooled Optically Stimulated Luminescence in $\mathrm{Al}_{2} \mathbf{O}_{3}$ for Ultrasensitive Radiation Detection
}

\author{
S. D. Miller and M. R. Tinker (Health Physics)
}

Cooled optically stimulated luminescence (COSL) has been successfully developed and characterized in $\mathrm{CaF}_{2}: \mathrm{Mn}$. In order to remain current in the state of the art, a number of new materials believed to be candidate COSL materials should be investigated. One such material showing a great deal of potential is $\mathrm{Al}_{2} \mathrm{O}_{3} . \mathrm{Al}_{2} \mathrm{O}_{3}$, in an initial test, has produced over 1,000,000 counts per $m R$ without optimizing any of the readout parameters, while $\mathrm{CaF}_{2}: \mathrm{Mn}$ has produced only 200,000 counts per $\mathrm{mk}$. With ad ional study, $\mathrm{Al}_{2} \mathrm{O}_{3}$ is almost certain to be many times more sensitive, while offering the advantage of requiring only modest cooling to perform readout. Since the COSL readout of $\mathrm{Al}_{2} \mathrm{O}_{3}$ can be done at higher temperatures than $\mathrm{CaF}_{2}: \mathrm{Mn}$, a simpler reader can be constructed using peltier coolers. The improved COSL readout process using $\mathrm{Al}_{2} \mathrm{O}_{3}$ may provide technology that will carry on the success of the previous COSL projects and be a useful addition to future environmental radiation monitoring efforts.

Cooled optically stimulated luminescence (COSL) is a method of optical readout of radiation detectors that utilizes the following three-step readout process. First the COSL material is cooled to below room temperature (in the case of $\mathrm{CaF}_{2}: \mathrm{Mn}$ to $77 \mathrm{~K}$ ), next the COSL material is illuminated with light, and finally the COSL material is warmed to room temperature after a brief wait. Luminescence proportional to the ionizing radiation exposure occurs as the COSL material warms to room temperature.
As part of a 2-year LDRD effort, two technical areas were developed this year 1) a suitable thermal annealing procedure for $\mathrm{Al}_{2} \mathrm{O}_{3}$, and 2) the optimization of the readout wavelength.

\section{Improved Annealing Procedure}

Annealing procedures are required for radiation dosimeters to remove residual signal before reuse. Annealing procedures used in thermoluminescence proved to be incomplete in removing the residual COSL signals in $\mathrm{Al}_{2} \mathrm{O}_{3}$. Much higher temperatures were used in an attempt to erase a larger portion of the residual signal. Note that the ultimate sensitivity of a radiation dosimeter is determined in part by the effectiveness of the annealing procedure. A temperature of $850^{\circ} \mathrm{C}$ was determined to be a sufficient to remove a large fraction of the residual COSL signal. It was a concern that this high temperature may damage the sensitivity of the material, but this did not occur.

\section{Readout Wavelength Optimization}

COSL readout can be accomplished by using a wide band of optical wavelengths. The optimal wavelengths where the maximum COSL sensitivity occurs must be determined in any new COSL material. To do this, a wide-band light source was fitted with various narrow-band interference filters and a fiber optic for delivery of the light to the sample. Wavelengths from $350 \mathrm{~nm}$ to $699 \mathrm{~nm}$ were used to measure the COSL efficiency and it was determined that $448 \mathrm{~nm}$ was the optimal wavelength for stimulation. 


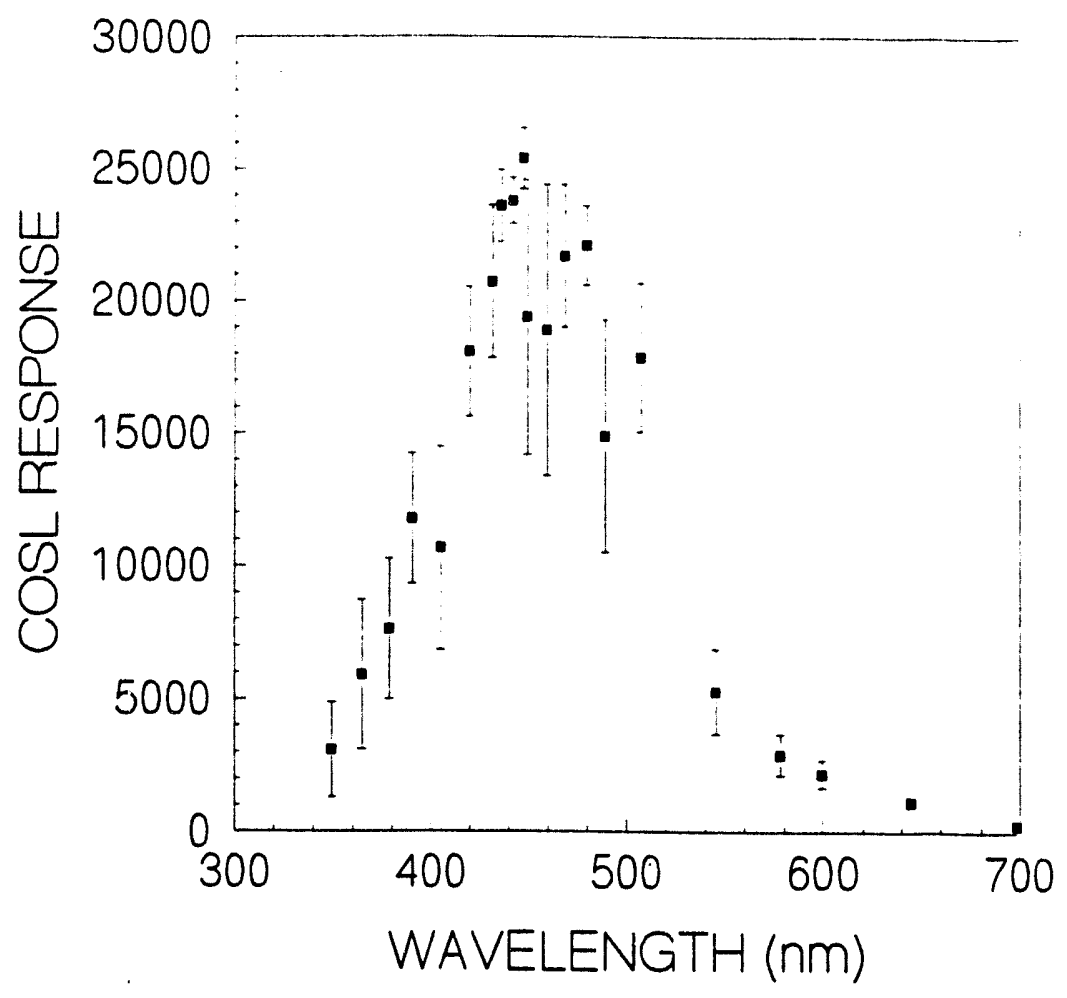

Figure 1. Wave Data - COSL Response in $\mathrm{Al}_{2} \mathrm{O}_{3}$ 


\title{
Development of a Ribbon Channel Plate for DNA Sequencing
}

\author{
R. J. Douthart (Life Sciences)
}

The overall objective of this research was to develop new technology for DNA sequencing and fingerprinting. This specific research involved development of a new gel electrophoresis technology that is a hybrid between gel-filled capillaries and ultra-thin gels. The new technology involves fabrication of a thin channeled plate. The plate consists of individual microchannels approaching capillary dimensions, etched or machined into it. Each channel is isolated from its neighbors by a thin wall and, therefore, supports an individual gel micro-ribbon. The plate, however, can be filled with gel at one time guaranteeing consistency in the micro-ribbons, with respect to composition and casting variables.

A gel assembly unit holds the plate casting and has built into it a thermostated baffle that allows very rapid heat exchange. The advantages of rapid heat loss with concurrent partial decoupling of running temperature and electrosensitive heating associated with gelfilled capillaries, and the ease of preparation and consistency of slab casting are incorporated in the ribbon channel plate.
Loss of cooling during a test run early in FY 1993 resulted in overheating and permanent damage to the apparatus. An adequate cooling device was then purchased and much of the apparatus rebuilt.

In spite of this setback, very meaningful results were obtained in FY 1993. Using m13mp8 vector and dideoxy sequencing, resolvable DNA sequence has been obtained with data density of $>7000$ points on standard $\mathrm{x}$-ray film.

Although resolvable, the sequence is not totally readable over its entirety. This is caused by point smearing due to constant drum speed and an insidious slow leak from the upper buffer chamber that travels down the sides of the plate to the drum plate assembly interface. The leak was traced to a crack in the upper plate that was repaired rather than building a new plate because of funding limitations. The drum speed can be varied with time by reprogramming the stepper motor. 


\section{Ethical, Legal, and Social Implications of the Human Genome Project for Screening, Monitoring, and Health Surveillance of Department of Energy Workers}

L. E. Sever (Epidemiology and Biometry)

The objective of this study was to examine a number of the implications of the Human Genome project for screening, monitoring, and health surveillance for Department of Energy workers. This was done by 1) reviewing relevart literature in genetics, ethics, law, and social policy;

2) holding discussions with experts from relevant disciplines; and 3) planning a white paper describing key issues. The white paper will provide the basis for additional activities related to policies and options regarding application of methods from the Human Genome project to DOE workers.

The accomplishments to date include the development and review of bibliographies relevant to legal and ethical aspects of genetic screening and monitoring programs. Extensive references have been obtained, reviewed, and abstracted. Particular attention has been paid to literature on discrimination and legal and ethical aspects of genetic screening programs.
Literature regarding exclusionary policies and legal implications of fetal protection policies has been reviewed as a possible paradigm for genetic screening policies.

Topic statements that explore the implications of genetic technology, in the areas of worker screening and monitoring, are being developed. These topic statements will provide the framework for a white paper.

Discussions were held with experts in the areas of genetics and bioethics to explore some of the issues involved in worker screening. In addition, discussions were held with PNL staff with relative interests to identify issues with particular relevance to programs directed by the DOE.

An outline for a workshop regarding the application of genetic screening and monitoring growing out of the Human Genome Project was drafted. The workshop was to focus on aspects of the Human Genome Project of particular relevance to DOE workers. 


\title{
Evaluation of Mercuric Iodide for Beta Radiation Detection
}

\author{
D. R. Sisk (Health Physics)
}

A mercuric iodide detector currently used for gamma spectroscopy was developed and evaluated for use in the detection of beta radiation. The effort concentrated on estimating the efficiency and energy dependence of mercuric iodide when measuring beta radiation. Although mercuric iodide is not known to have been used as a beta detector, there is no evidence to indicate that it cannot be used in beta radiation detection. The objective in this study was to determine if the performance of mercuric iodide is such that it can replace gas-filled detectors.

In order for the testing of mercuric iodide $\left(\mathrm{HgI}_{2}\right)$ to begin, it was necessary to obtain two detectors (in different configurations) and a detector test box. The level of effort required to acquire the necessary materials for the project was much greater than expected. DOE-Nevada had reservations in providing PNL with the equipment, especially regarding ownership of patents and technology transfer responsibilities of any developments. The problems were resolved after about 3 months. Delays were also encountered in receiving the detectors, and they were not received until October 29. The receiving date was past the end of the project.

However, several critical measurements were performed during off-hours and some important observations resulted. The preamplifier in the detector test box provided by EG\&G, was well constructed, but was susceptible to noise induced by vibration. Therefore, care had to be taken to insulate the test box from mechanical and acoustic vibration. The sensitivity could be improved during design of a commercially viable instrument.

The $\mathrm{HgI}_{2}$ detector evaluated was approximately $2.5 \mathrm{~cm}$ by $2.5 \mathrm{~cm}$ square and $2 \mathrm{~mm}$ thick. The detector had palladium contacts and lead wires and was mounted on a ceramic substrate. The entire detector was encapsulated with a thin layer $(20 \mu \mathrm{m})$ of parylene. Pulses from the preamplifier had a time constant of approximately $0.45 \mathrm{msec}$. The time constant appeared to be the same for both gamma and beta radiation sources. Pulse shape discrimination was one method proposed earlier to differentiate between gamma and beta radiation. This method does not look promising in light of these observations.

Illustrations of measured spectra of beta and gamma radiation sources are attached. The measure spectra of the Sr-90/Y-90 $\left(\mathrm{E}_{\max }=2.2 \mathrm{MeV}\right)$ and Tc-99 $\left(\mathrm{E}_{\max }=0.290 \mathrm{MeV}\right)$ beta radiation sources indicates that $\mathrm{HgI}_{2}$ can detect beta radiation efficiently. The measured spectrum of the Cs-137 gamma radiation source reflects only Compton interactions by th Cs-137 gamma rays indicating that the volume of the $\mathrm{HgI}_{2}$ detector is too small to result in the generation of a significant number of full energy peaks.

Additional measurements will be conducted to continue evaluation of $\mathrm{HgI}_{2}$ as a detector for beta radiation. The results of the evaluation will be used in proposals to obtain programmatic funding in FY 1995. 


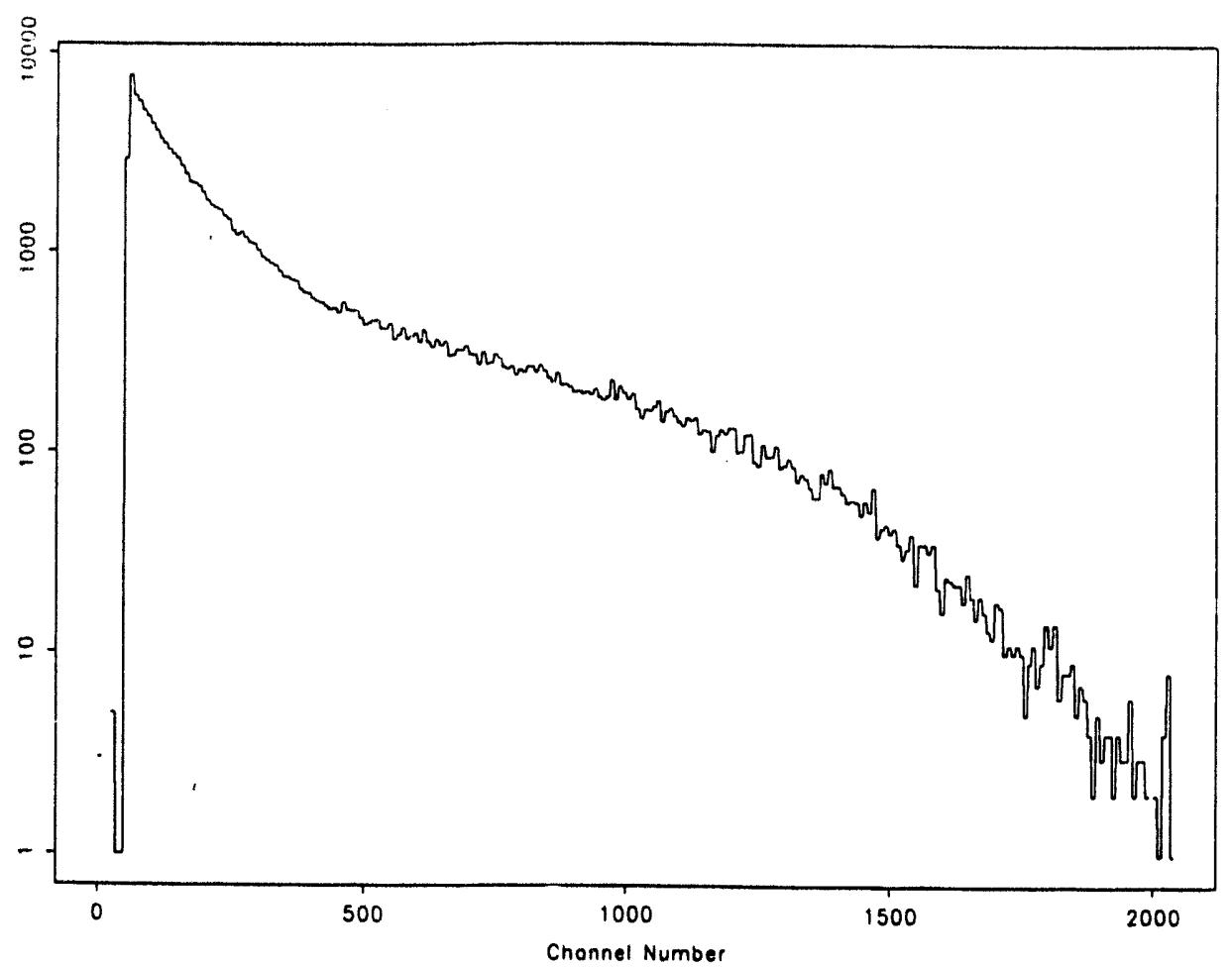

Figure 1. Spectrum of Sr-90/Y-90

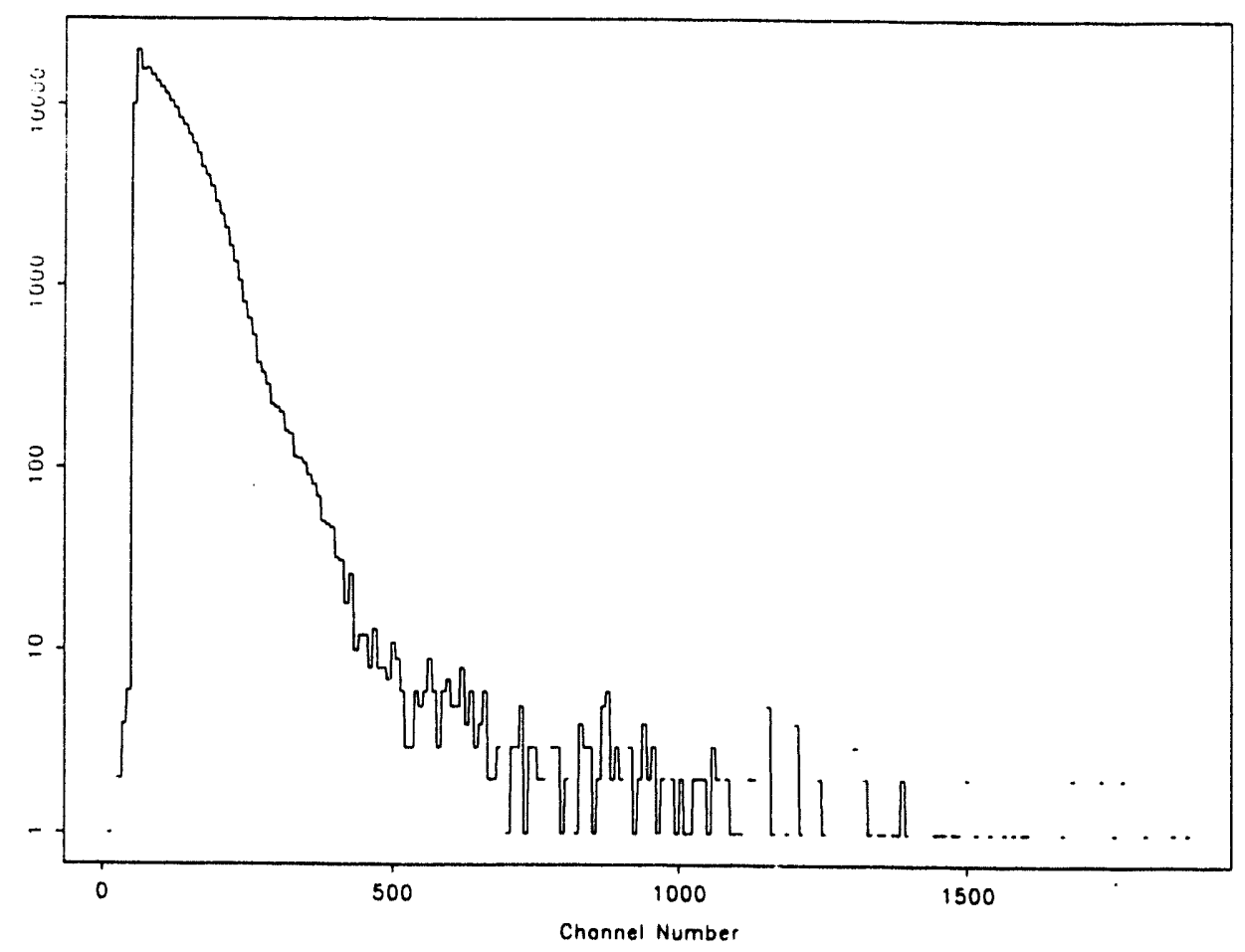

Figure 2. Spectrum of Tc-99 


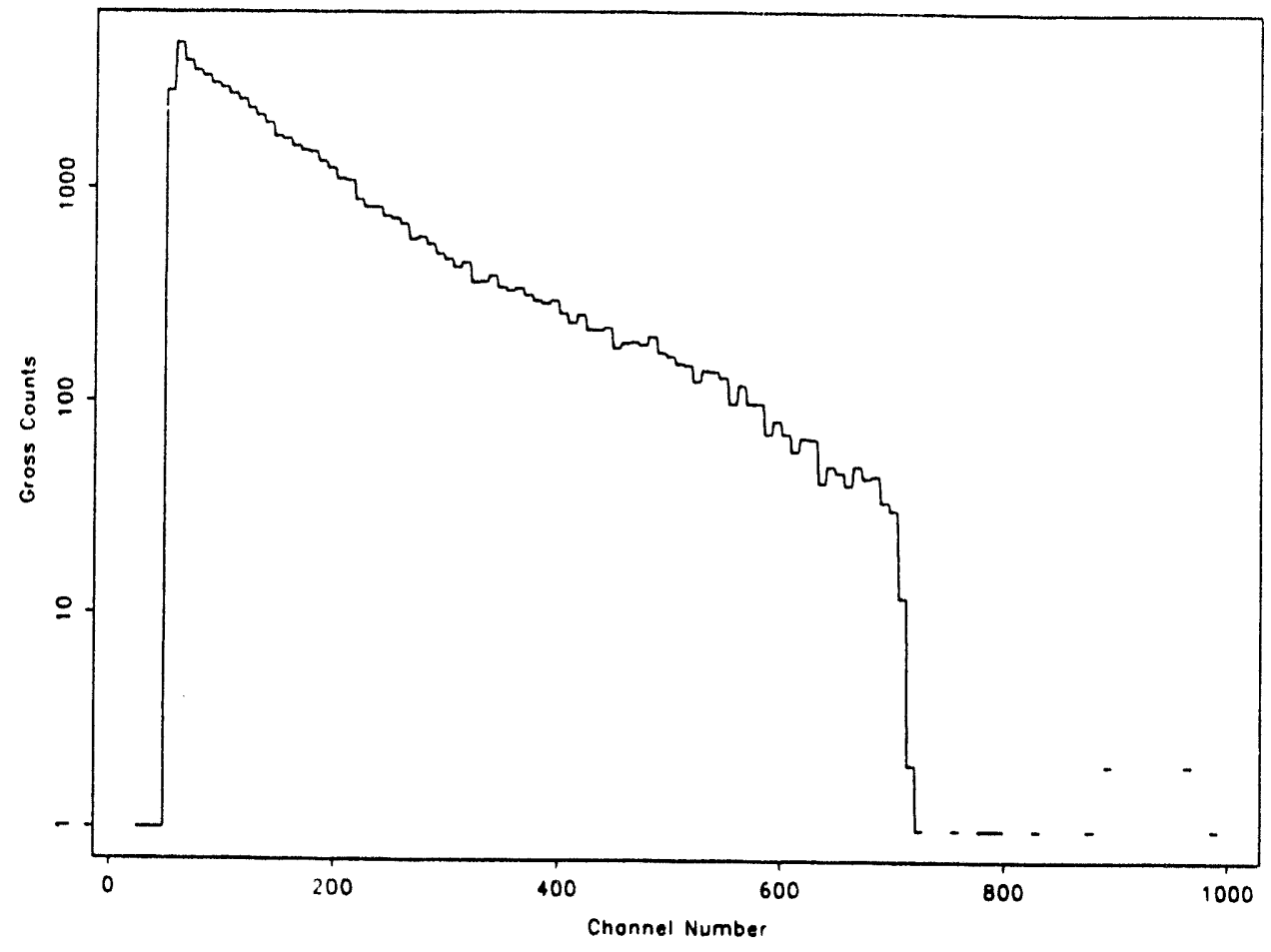

Figure 3. Spectrum of Cs-137 


\title{
Modeling of Images of the Human Body for Applications in Absorbed Dose Calculations
}

\author{
G. Akabani (Health Physies)
}

The use of images of the human body has contributed extensively to new advances in radiation dosimetry. The research impact in the area of radiation dosimetry is extensive and well supported by the types of applications being studied. With the advent of faster computers, it is possible to implement these new techniques by means of Monte Carlo transport on a routine basis.

\section{Imaging Technologies and Supercomputers}

The advent of new imaging techniques along with faster computers has allowed researchers to assess radiation doses in specific regions of the human body. Images of a specific patient or subject are used to assess energy deposition patterns for different sources of radiation commonly used by the medical community. The objective of this research was to develop and implement the required technology to apply radiation Monte Carlo transport for alpha, electron, and photons for the assessment of energy deposition patterns in specific regions of the human body. Images of the human body were generated and stored using the Hierarchical Data Format developed by the National Center for Supercomputing Applications (NCSA). This data format allows for the threedimensional representation of images of the body for further manipulation in large computer codes for purposes of Monte Carlo transport. Currently, there is an extensive effort carried out to assess localized doses, dose distributions, and dose volume histograms in tumors and other specific regions which require modeling and simulation prior to clinical treatment. This is to assess the probability of tumor control and tissue complication probability in patients. Imaging techniques developed during this work were implemented and used to assess energy deposition patterns in two specific regions of the human body of interest by the medical community. These regions were the bone marrow and lung.

\section{Bone Marrow Dosimetry}

For the case of bone marrow, imaging techniques were applied to assess dose distributions in biopsy samples for specific radionuclides by determining the local chord length distribution of bone marrow and trabecular bone. Furthermore, a detection system was designed to assess the radionuclide distribution (see Figure 1) for the source and target regions and determine the attachment efficiency of entities such as MAb into marrow cells. Figure 2 shows a cross section of a bone sample from which localized absorbed doses were obtained to assess the dose distributions in bone marrow itself. These distributions have microdosimetric information that can lead us to understand the cell killing efficiency of certain radionuclides for marrow ablation.

\section{Lung Dosimetry}

Lung images were used to model the tracheobronchial tree to assess the dose to the lung by noble gases used in diagnosis procedures. This diagnostic procedure has been used for many years for the detection of several pulmonary illnesses. Doses to the tracheobronchial tree were calculated for each specific branch of the lung based on the local lung structure. These techniques have been implemented previously into the medical area of radiosurgery of small brain tumors and now are being implemented in the small scale dosimetry area.

Publications

Akabani, G. "Localized Bone Dosimetry using Imaging Techniques." Journal of Nuclear Medicine, in press.

Akabani, G., A. C. James, A. Birchal, and J. Alvarez. "A Dosimetric Lung Model for Noble Gases." Journal of Nuclear Medicine, in preparation. 


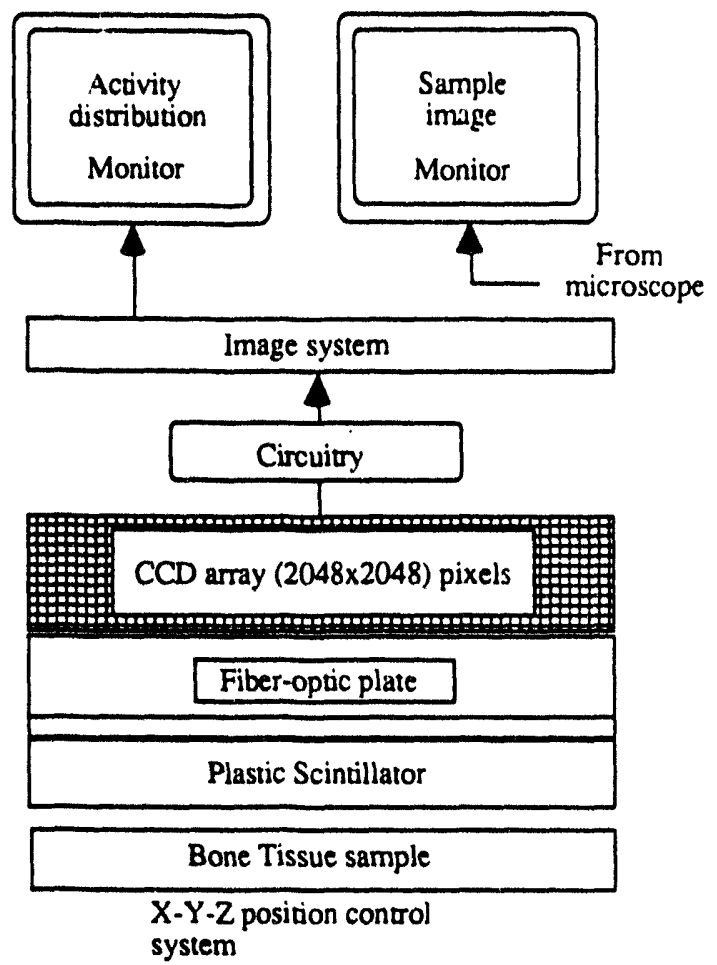

Figure 1. Detection System Designed to Assess Radionuclide Distribution

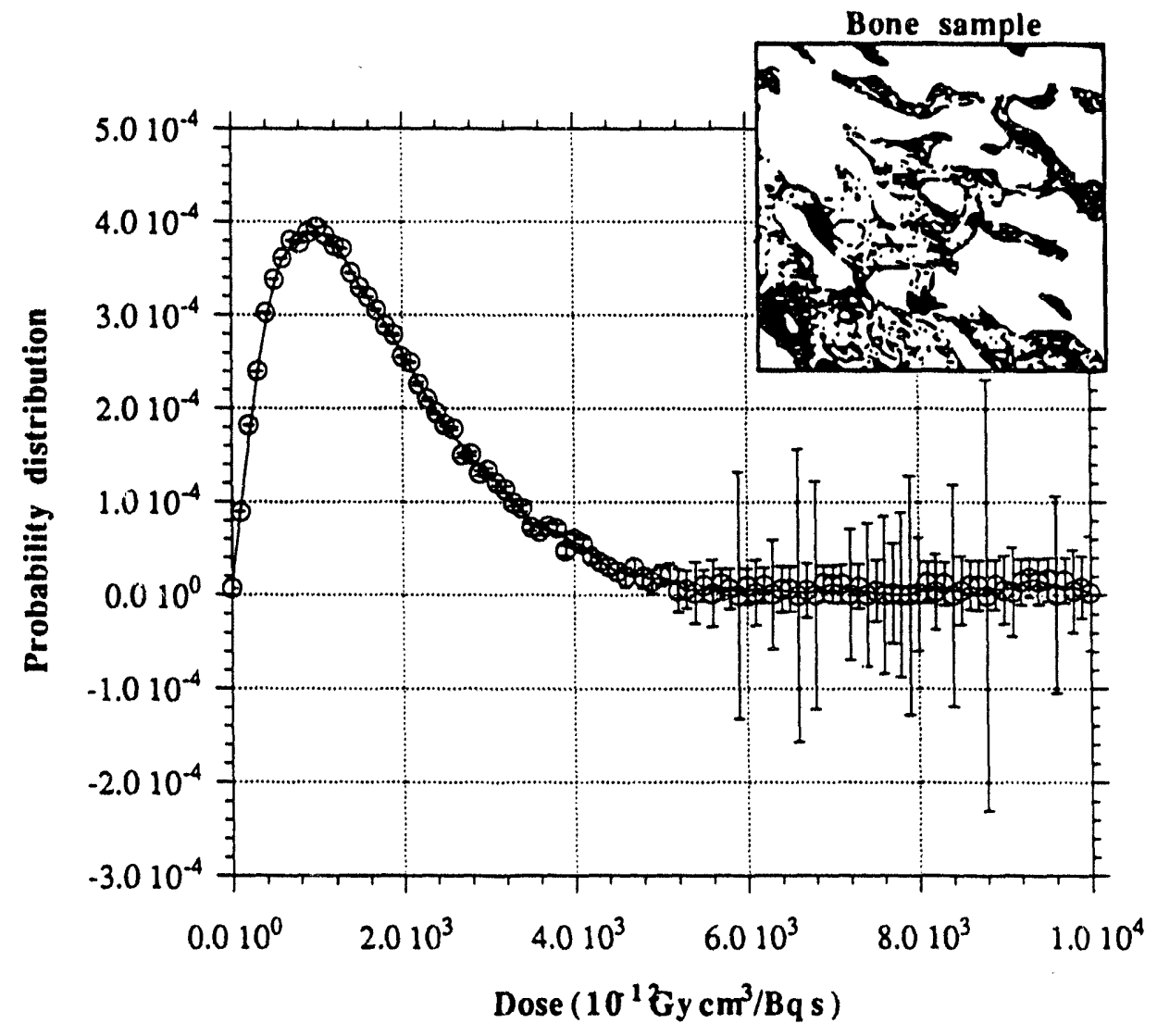

Figure 2. Probability Distribution of Absorbed Dose to the Bone Surface for a Uniform Distribution of Sr/Y-90 in Marrow Cavities for the Sample Bone Shown at Top of the Figure 


\title{
Non-Ionizing Radiation (NIR) Hazard Assessment Software
}

\author{
M. H. Smith (Health Physics)
}

Development concentrated on a software program that will help health physicists and industrial hygienists assess the non-ionizing radiation hazards of radiofrequency and microwave emitters. The program created is called SARAH (S Safety Analysis of Radiofrequency/Microwave Antenna Hazards).

SARAH calculates power densities for circular aperture antennas and compares these values to the guidance given in ANSI/IEEE Standard C95.11992 "Safety Levels with Respect to Human Exposure to Radiofrequency Electromagnetic Fields, $3 \mathrm{kHz}$ to $300 \mathrm{GHz}$."

During FY 1993, work on this task focused on the completion of the SARAH code. This non-ionizing radiation hazard assessment code is designed to operate on any DOS-compatible personal computer with a math co-processor.

In FY 1992 and FY 1993, work was also done with the Numerical Electromagnetic Reflector Antenna (NEC-REF) code written by Ohio State University. However, severe problems were encountered when the code would not compile and run adequately on different system platforms.
The current version of the SARAH code uses userdefined parameters to estimate power density generated by a circular aperture antenna. In addition to calculating power density at a user-specified location, SAPAH also estimates power density at the near field, intermediate field, and far field boundaries. The output generated by SARAH includes the ANSI/IEEE C95.1 limits for radiofrequency and/or microwave exposure in controlled and uncontrolled areas, an estimate of minimum distances for unrestricted exposure in these areas, and an estimate of allowed residence time in areas that would require restricted exposure.

The SARAH code has been used in the field by PNL personnel to estimate non-ionizing exposure prior to taking measurements near antennas that could pose a hazard to human health or damage sensitive measurement equipment.

\section{Other Accomplishments}

The code was demonstrated during a poster session at the annual Health Physics Society meeting in Atlanta, Georgia. An invited demonstration of the code was given at the "NonIonizing Radiation Survey Training" class sponsored by Narda Microwave, Inc. 


\title{
Physiologically Based Pharmacokinetic Modeling of Hazardous Chemicals
}

\author{
T. E. Hui (Health Physics)
}

A physiologically hased pharmacokinetic (PBPK) model was developed for a specific volatile organic chemical. This was expedited by the development of a generic mathematical computer code designed to solve PBPK models.

A real challenge in worker protection is to identify and monitor biomarkers of exposure to toxicants when the worker is potentially exposed from various types of activities. Currently, there are no external means of measuring the magnitude of the total integrated exposure. Epidemiologists have long sought some scientific link between actual chemical exposure and observable adverse health effects. The ability to match the extent of exposure with biological indicators can be accomplished with the use of simulation models. The development of these integrated models enables one to design mechanistic toxicology studies that maintain a human health risk assessment focus, and to integrate these aspects more fully into the risk assessment process.

The strategy of PBPK modeling is to calculate toxicant/metabolite concentrations within target tissues and that excreted (via exhalation, urine, feces, and/or that transpired from the skin), and to quantify occupational exposure based on measurements of contaminants in the environment/work place and bioassay data. Ultimately, this information can be used to predict adverse health outcome or susceptibility to disease, and to quantify cancer risk from exposure.

The detailed kinetic data needed to develop a valid PBPK model is generally obtained from animal studies. However, with appropriate modifications, the animal model can be extrapolated to predict human response. If sufficient kinetic information is available, PBPK models can be used to extrapolate from high to low dose, from a single acute exposure to repeated exposures, and from continuous to intermittent exposures. A PBPK model has the sensitivity to deter- mine the quantitative relationships between different biomarkers following unusual exposure situations.

In this study, a PBPK model was developed to characterize the maternal and fetal tissue uptake and retention of methyl mercury.

Methyl mercury was the chemical selected to be studied. In order to keep the model simple and thus more complete, preference was given to a chemical that does not undergo substantial internal metabolism, particularly secondary metabolism. Furthermore, the chemical needed to be one in which a large amount of biokinetic data was already available, collected from both experimental animals and limited human studies. Methyl mercury met those criteria.

During FY 1993, a PBPK model was developed to describe the uptake and tissue distribution of methyl mercury in the pregnant rat and fetus. This model is at a stage in its development where additional experimental studies are needed to improve model predictions and a follow-on proposal is planned to be submitted for these studies. It is anticipated that this same method of model development, identification of data deficiencies, and preparation of a proposal for additional funding, could be applied to a different chemical or chemical mixture.

The common physiological parameters used in PBPK modeling were consolidated, based on a literature review of published data. A summary of the findings was submitted for journal publication. We have begun to demonstrate our expertise in PBPK modeling, as described in the summary of prior accomplishments. Recently, we have installed a software package, Simusolv, on a SUN SPARC10-41 workstation (both purchased under different projects). The acquisition of these tools greatly enhances our capability in PBPK modeling.

In FY 1994, we plan to develop PBPK models for another chemical, or a simple mixture of chemicals. 


\section{Publication}

Thrall, K. D., and T. E. Hui. "A Review of the Physiological Parameters Used in Recently Published Physiologically Based Pharmacokinetic Models." Joumal of Toxicology and Environmental Health, submitted.

\section{Presentations}

Cappas, M. C., K. D. Thrall, T. E. Hui, and J.A. Leonowich. "A Physiologically Based Pharmacolinetic Model for Methyl Mercury in the Pregnant and Fetal Rat." To be presented at the American Industrial Hygiene Conference and Exposition '94, May 21-27, 1994, Anaheim, California.

Thrall, K. D., A. C. James, and T. E. Hui. 1993. "Physiologically Based Pharmacokinetic Modeling of Radon, Thoron, and Their Short-Lived Progeny." Presented at the Society of Toxicology Annual Meeting, New Orieans, Louisiana.

\section{Other Accomplishments}

In collaboration with Dr. Alan Birchall of the National Radiological Protection Board in the United Kingdom, we developed a mathematically based computer code to aid in the simultaneous optimization of parameters for solving a generic linear PBPK model (GIGAFit or Graphically Interactive General Algorithm for Fitting).

Mentored Michael Cappas, a DOE Industrial Hygiene Fellow from the University of California at Los Angeles, who performed his practicum at PNL doing his thesis work on this project. Based on his work on the PBPK modeling of methyl mercury, he has presented a seminar in the Health Physics Department, and is preparing a journal article. 


\title{
Properties of Soft X-Ray Absorption and DNA Damage by Syncrotron Radiation
}

\author{
L. A. Braby (Biology and Chemistry)
}

\begin{abstract}
The biological effects of ionizing radiation are initiated by physical and chemical changes induced in cellular DNA. The nature of these changes and their concentration in DNA is influenced by the spatial pattern of the energy deposited by the ionizing particles delivering the dose. Understanding the local characteristics of damage to cellular DNA by ionizing radiation is a key ingredient in understanding the origins of the enhanced relative biological effectiveness of highlinear-energy-transfer radiation. This work will exploit the special characteristics of synchrotron radiation and specially prepared DNA plasmids to investigate the properties of initial damage to DNA as a function of electron energy (i.e., electrons with energies from approximately 200 to $800 \mathrm{eV}$ ). These short-range photoelectrons provide an excellent probe of the effect of energy localization on DNA damage.
\end{abstract}

After investigating several alternatives, it became evident that the most appropriate synchrotron source for these radiation damage studies is the Synchrotron Radiation Center at the University of Wisconsin. This machine produces the photon energies needed, the 200 to $800 \mathrm{eV}$ range, at high dose races, without the problems of higher energy contamination which occur when a more energetic storage ring is used. Furthermore, there is a beam line specifically set up and in use for cell irradiations at this facility.

In order to determine the requirements for a plasmid irradiation experiment using this source, we visited Dr. Michael Gould at the University of Wisconsin, and checked on the details of the cell irradiation procedure. The $x$-ray monochromator produces a narrow horizontal beam about 1.5 inches long. The target cell, a special petri dish with thin plastic film bottom, is scanned past the beam by a system which adjusts the scan rate for the storage ring current and desired dose. Several petri dishes can be held in the scanner so that it does not require opening the irradiation system to change dishes during a typical experiment. Laboratory space and some facilities are available near the light source so that we will be able to complete our radiation damage studies at the site.

Since it is difticult to modify an experimental procedure at an offsite accelerator, and because we need to compare the effects of 200 to $800 \mathrm{eV}$ photons with those of protons and alpha particles, we will test our irradiation procedures with beams from the PNL tandem accelerator before attempting experiments in Wisconsin. To this end, we have designed a target cell that will fit both the Wisconsin machine and a modified version of the tandem beam line which we have used for oriented DNA irradiations. This cell consists of a $1.5 \mu \mathrm{m}$ plastic film held by a pair of stainless steel rings. The DNA solution is placed on a plastic piston and moved into contact with the film. Surface tension causes the solution to spread to a uniform thin layer, ideal for irradiation by soft $x$-rays or charged particles. After irradiation the piston is removed and the sample coalesces into a small droplet, which can be picked up by a micropipet. This system has been tested to demonstrate recovery of the sample and a sample cell has been sent to Wisconsin for testing in their irradiation system.

The PNL tandem accelerator beam line is being renovated and modified to conduct plasmid irradiations. The sample holder used for oriented DNA samples has been replaced with one to hold the plasmid irradiation cells. The original Faraday cup and shutter mount are being modified to improve the reliability of the equipment and therefore the accuracy of the results. During the oriented DNA experiments we learned that these components require a significant amount of maintenance, and the original cesign required realignment of the beam line whenever work was done on the shutter or cup. These modifications are under way, the design has been completed and parts fabricated, although some standard vacuum components have not yet arrived. 


\title{
Radiation Damage and Repair in Mammary Carcinogenesis
}

\author{
K. M. Groch and A. L. Brooks (Biology and Chemistry)
}

Breast cancer is a significant health risk to women following exposure to ionizing radiation. An important question in the pathogenesis of radiation-induced breast cancer is the cellular and molecular basis for the tissue-specific nature of the disease. We proposed to examine the repair of radiation-induced chromosome damage by rat mammary epithelial cells obtained from animals of three strains which differ in intrinsic susceptibility to mammary cancer, and compare these data to those obtained from other epithelial cells obtained from the same strains of rodents.

Substantial progress was made during FY 1993 on this project. The laboratory infrastructure required for the performance of the work was established in the 331 Building. The methodology for tissue dispersion and the tissue culture systems for both types of epithelial cells was established. These efforts included

- obtaining and preparing the various growth factors and hormones for the respective tissue culture media
- obtaining the enzymes, ancillary reagents, and laboratory equipment for the isolation of rat mammary and tracheal epithelial cells.

As expected, both rat mammary and tracheal epithelial cells were successfully isolated on several occasions and primary cultures established.

Ms. RoeAnn Barker, a DOE Energy Research Fellow under the direction of Dr. Brooks was instructed on all components of the work including the procurement of tissues from experimental rodents, the isolation of dispersed cells for culture, and the culturing of the dispersed cells. As a direct consequence of her efforts, molecular chromosome paints specific for rat chromosomes $1,2,4,10, X$, and $Y$ were prepared in collaboration with Lawrence Livermore Laboratory.

A total of five competitive proposals based on the system were prepared and submitted for external funding. 


\title{
Use of Proliferating Cell Nuclear Antigen (PCNA) Assay to Quantify Cell Proliferation in Respiratory Tract of Rodents Inhaling Chemicals or Radioactive Materials
}

\author{
R. A. Renne (Toxiocology)
}

The objective of this project was to improve our capability to identify and quantify cells proliferating in target tissues of laboratory animals as a result of exposure to radiation or toxic chemicals, and utilize this information to study the relationships between cell proliferation and cancer induced by physical or chemical insults.

In FY 1993, we further refined the assay utilizing a monoclonal antibody against proliferating cell nuclear antigen (PCNA) to measure cell proliferation in the respiratory tract of rodents inhaling xenobiotics. We performed the proliferating cell nuclear antigen assay on nasal mucosa of rats inhaling a test chemical for 18 months, and compared the results in exposed and control groups using two different fixatives. We also developed and refined techniques which will allow us to further utilize our image analysis instruments and computer software to automatically count labeled and unlabeled cells in respiratory tract and other tissues using light microscopy and a digital camera. Much of this progress was made possible through the addition of a NORCUS graduate student, Ms. Kim Saunders, who spent 3 months in our laboratory and played a key role in the proliferating cell nuclear antigen assays done on rats in the study described above. She also participated in upgrading our image analysis software to make counting of labeled nuclei more accurate and faster. A poster describing our latest proliferating cell nuclear antigen studies on rat nasal mucosas was presented at a symposium on mechanisms of nasal toxicity and carcinogenesis in Research Triangle Park, North Carolina, September 20-23, 1993. The proceedings of this conference, including extended abstracts from the poster presentations, will be published in Inhalation Toxicology.

\section{Other Accomplishments}

During FY 1993, we presented a poster at the Society of Toxicology annual meeting comparing the BRDU and proliferating cell nuclear antigen data from our glutaraldehyde instillation study done earlier. We also made further progress on preparation of the manuscript describing this work; this manuscript is now nearly ready for submission. 
2.4 Materials and Chemical Sciences 


\title{
Advanced Nanometer-Scale Electronic Materials Syntresis
}

\author{
J. C. Linehan (Chemical Sciences)
}

The objective of this project was to lay the groundwork for large-scale production of nanometersized electronic, optical, and advanced ceramic and metallurgical materials.

Nanoscale building blocks for advanced materials have been thought to be superior to micron-size powders for a variety of reasons. These include lower processing costs due to lower sintering temperatures, more ductile and higher strength ceramics, catalysts with higher activities, electronic materials with superior properties. Experimental testing of these properties on actual nanoscale materials has been thwarted by the lack of methods of producing nanoscale powders in bulk. Recently, two new PNL-developed technologies, modified reverse micelle (MRM) and rapid thermal decomposition of precursors in solution (RTDS), have been used to successfully produce large quantities of previously unavailable nanoscale powders. The RTDS method allows large quantities of oxide and oxyhydroxide single phase or multiphase powders to be continuously produced in water. Crystallite sizes (from 1 to $20 \mathrm{~nm}$ ) of the powders can be controlled by reaction temperature and residence time. The modified reverse micelle process allows more fine control over the powders produced as well as direct access to metallic and reduced forms of the powders. These two methods have been utilized to produce new materials with improved capabilities for a variety of applications.

\section{Advanced Nanoscale Capacitors}

The ability to produce nanometer-sized materials with multifunctional components is essential for advanced electronics. In particular, the use of bi-layered nanoscale materials may allow for further miniaturization of electronic devices with increased capacities. The modified reverse micelle process was shown capable of producing the various components necessary for a bi-layered spherical nanometer-size particle. The necessary conditions for silica formation, i.e., $\mathrm{pH}$, concentration, cemperature, etc., in the modified reverse micelle system were tabulated. The conditions for metallic nickel powder and for cadmium sulfide formation on the aqueous cores of the modified reverse micelle were also investigated. The conditions for the formation of a nanometer-size spherical capacitor consisting of a metallic nickel core, insulted with silica and coated with cadmium sulfide are known.

\section{Advanced Catalysts}

The RTDS process allows the productions of ultrafine, nano-crystalline, high surface area materials with excellent catalytic properties. The ultrafine size of the RTDS-produced catalyst offers several advantages over sther catalyst powders. These include better diffusivity of the catalysts throughout the reaction mixture and a larger number of active sites per unit weight of the catalyst.

The production of advanced catalysts by the RTDS method were successful. Two different catalyst systems were successfully produced by the RTDS method. The RTDS method provided a flexible synthesis method allowing for incremental changes in the properties of catalysts produced. Even small changes in the RTDS conditions led to large differences in the catalyst size, surface area, or actual phase produced. The changes in the catalyst structure also resulted in large changes in the catalytic activity exhibited. Some materials produced by RTDS were found to show superior activity when compared to similar materials produced using conventional methods. The two different catalyst systems were shown to be active for two completely different catalytic processes. There are now five catalyst systems which have been successfully synthesized by the RTDS process. These catalysts have all shown superior qualities over conventionally nroduced catalysts. Optimization of the production of the two catalysts materials discussed here was initiated.

\section{Summary}

Several new applications of the RTDS and modified reverse micelle nanoscale material producing methods have been identified and tested. The two ultrafine particle producing technologies will continue to be used in follow-up studies based on the results obtained in this investigation. 


\section{Catalyst Design}

M. A. Lilga and T. L. Hubler (Chemical Technology)

Novel bimetallic chromium complexes have been prepared and hydrogenation and isomerization catalysts investigated in LDRD-funded research. Dienes are selectively hydrogenated to monoenes. In this project, the nature of the catalytic reactions and the roies of the catalysts were probed. This work will help to advance the state of the art in catalysis science.

The dinuclear hromium complex 1 , in which two cyclopentadienylchromium moieties are coupled by a methylene linkage, has been observed to reversibly bind $\mathrm{H}_{2}$ (Equation 1). When the bridging methylene group is absent, as in the dinuclear chromium complex 3, the uptake of $\mathrm{H}_{2}$ is irreversible and results in fragmentation of the molecule into two mononuclear fragments (Equation 2). Hence, a unique situation exists for 1 in which increased interactions of the metal centers, brought about by the bridging ligand, results in reversible binding of $\mathrm{H}_{2}$. For 1 , the possibility of cooperative interactions between the two metal centers exists and may affect the hydrogenation catalysis.

The objectives of this project were to characterize the bridged chromium dimer, 1, to investigate the hydrogenation catalysis of both the bridged and unbridged complexes, and to investigate the kinetics of $\mathrm{H}_{2}$ binding by both complexes.

Complete spectroscopic characterization of complex 1 by means of ${ }^{1} \mathrm{H}$ and ${ }^{13} \mathrm{C}$ nuclear magnetic resonance, infrared, ultraviolet/visible, mass spectrometry, and $x$ ray diffraction methods has been performed. Additionally, variable-temperature nuclear magnetic resonance studies demonstrate the fluxional nature of 1 in solution. The fluxional motion consists of torsional twisting of the cyclopentadienyl rings relative to the methylene bridge. A crystal structure determination of 1 was also achieved.

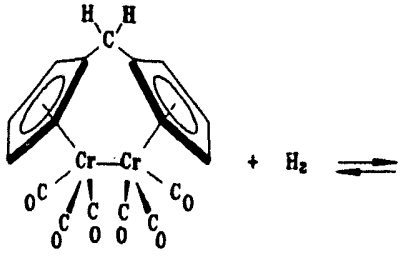

1

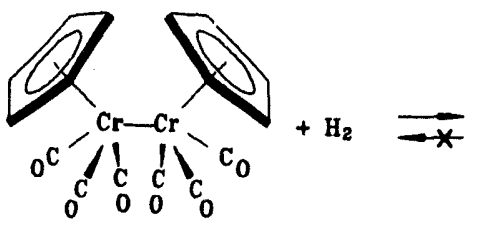

3

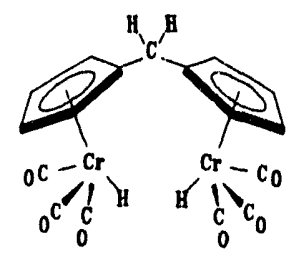

2

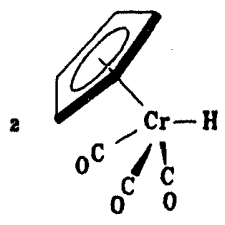

4
Complexes 1 and 3 hydrogenate butadiene to give a mixture of butenes, with hydrogenation by the unbridged complex 3 occurring at a rate twice that observed for 1 . These studies have shown that both complex 1 and 3 catalyze the specific hydrogenation of conjugated dienes to give internal olefins. The specificity to mono-olefins and the observation that isomerization of olefins does not occur suggests that hydrogenation may occur by a free radical process. Further research would be needed to confirm this possibility.

Kinetic studies for $\mathrm{H}_{2}$ uptake by the two complexes were conducted utilizing ultraviolet/visible spectroscopy. For the reaction of the bridged complex 1 with $\mathrm{H}_{2}$, the reaction is first-order with respect to 1 and first-order with respect to $\mathrm{H}_{2}$. The rate constant for the bimolecular reaction is $0.2 \mathrm{M}^{-1} \mathrm{~s}^{-1}$. A typical series of spectra taken as a function of time is shown in Figure 1 and shows clean replacement of 1 by 2 (note the isosbestic points). In contrast, uptake of $\mathrm{H}_{2}$ by the unbridged complex 3 appears to proceed through an intermediate species as indicated by a redshift in absorbance prior to disappearance of the absorption band. The disappearance of the band in 
the visible spectrum attributable to the intermediate species is second-order (a plot of 1/absorbance versus time is linear) and is independent of the $\mathrm{H}_{2}$ concentration (zero-order in $\mathrm{H}_{2}$ ). Thus, the kinetic results indicate that the reactivity of complex 1 is much different than that of 3 and is a direct result of the effects imposed by the methylene bridge of the ligand.

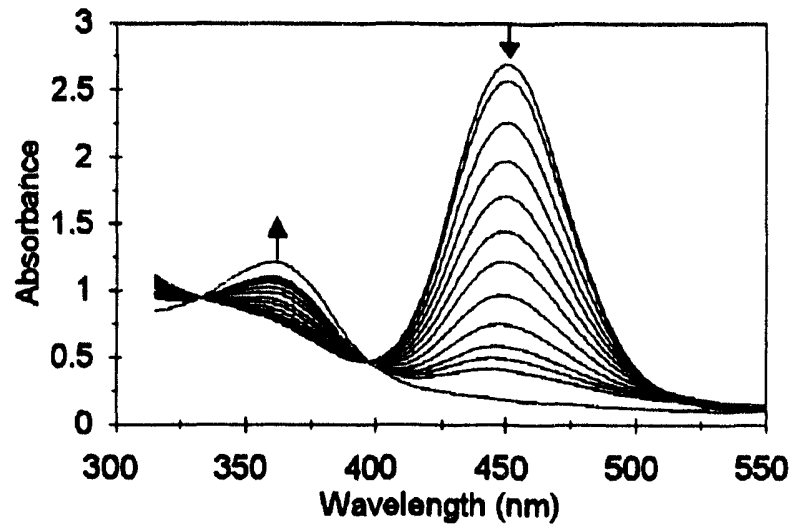

Figure 1. Absorption Spectra Taken During the Reaction of the Bridged Chromium Dimer, 1, with $\mathrm{H}_{2}$. 


\title{
Catalyst Development for High-Temperature Pressurized Aqueous Chemical Processing Systems
}

\author{
T. A. Werpy (Chemical Technology)
}

Catalytic hydrothermal processes have been shown to be effective for the conversion of biomass to gaseous fuels and for the destruction of hazardous organic compounds in aqueous waste streams. In order for these processes to continue to develop and become commercially viable, new catalysts must be developed which can withstand the hightemperature pressurized liquid water environments. Commercial catalysts have been tested and suffer from poor support stability under these environments. The objective of this work is to develop catalyst supports based on hydrous metal oxides ion-exchangers. Hydrous metal oxides ionexchange materials afford the opportunity to develop, catalysts which will be highly stable under high-temperature pressurized liquid water environments.

Several different layered titanates were synthesized for application as catalyst supports for nickel and ruthenium. Nickel and ruthenium have been shown to be active metal catalysts for application in the hydrothermal conversion of waste organics to methane and carbon dioxide. The synthesized layered titanate support materials were characterized by $x$-ray diffraction and BET surface area measurements. X-ray diffraction was used to confirm the formation of the layered structure of the titanates. Metal loading capacities of the layered materials were estimated from the results of BET surface area measurements. Typical BET surface areas of the layered titanate support materials were $250 \mathrm{~m}^{2} / \mathrm{g}$. Active catalysts were prepared by impregnating the host titanate with either nickel or ruthenium metal, followed by thermal treatment at temperatures up to $400^{\circ} \mathrm{C}$. Surface area measurements were employed to evaluate the thermal stability of the titanate catalysts. Typical surface areas of the thermally treated materials were $200 \mathrm{~m}^{2} / \mathrm{g}$. X-ray diffraction patterns showed that the metal loaded titanate catalysts remained layered after the thermal treatment. Catalysts were prepared with nickel loadings between $2 \%$ and $10 \%$ on a weight basis. Ruthenium catalysts were prepared between $2 \%$ and $5 \%$ on a weight basis. All of the catalysts prepared were then reduced in hydrogen for 24 hours prior to catalytic testing. The catalytic testing of the metal impregnated titanate catalysts was carried out in a 1 -liter batch reactor. The reactor was equipped with both liquid and gas sampling ports which allowed continuous removal of the reaction products for analysis. The reactor was charged with 35 grams of the selected catalyst and a predetermined amount of p-creosol. Analysis of the reaction products included monitoring the formation of both carbon dioxide and methane. Reaction conditions were $350^{\circ} \mathrm{C}$ and approximately $3000 \mathrm{psi}$. The test results for the destruction of p-creosol using both the nickel and the ruthenium impregnated catalysts showed significantly less activity than the conventional nickel and ruthenium impregnated catalysts. The ruthenium catalysts showed some initial activity; however, the activity decreased significantly after a very short time. Characterization studies carried out after the catalytic reaction showed that the layered structure of the titanates had been destroyed during the reaction. The nickel and ruthenium catalysts recovered after catalyst testing yielded BET surface area values of only 25 to $50 \mathrm{~m}^{2} / \mathrm{g}$. Based on the results of the $\mathrm{X}$-ray diffraction studies and surface area studies, it is evident that the layered titanates cross link under catalytic reaction conditions, causing significant loss of surface area. The significant loss in surface area and structural integrity reduces access of the reactant molecule to the active metal on the catalyst. The loss of accessibility to the active metal accounts for the poor performance of the catalyst in the destruction of the p-creosol to methane and carbon dioxide. Based on the results of this study, it is unlikely that the layered titanate materials will be useful as a catalyst support for either nickel or ruthenium for the catalytic destruction of organic waste. 


\title{
Design, Development, and Characterization of Polymer/Glass Composites
}

\author{
G. J. Exarhos (Materials Sciences)
}

A new route to the preparation of binary molecular composites is proposed, based upon intimate mixing of precursor species in solution followed by solvent removal and thermal processing to achieve homogeneity. This research involved the design and synthesis of precursor reagents selected to attain mixing on a molecular scale; refinement of processing techniques to achieve a target microstructure; and characterization of the resulting composite with respect to thermal and mechanical stability, glass transition temperature, and chemical bonding between the constituents. Target materials are expected to exhibit superior properties over existing polymer-glass composites, which are produced by extrusion methods.

Several years ago, Corning Glass patented a process in which a high $\mathrm{T}_{\mathrm{g}}$ organic polymer could be extruded with a low melting temperature phosphate glass to form a tough composite material having applications as a structural composite and as a catalyst support material. Ongoing work at PNL regarding the development of new inorganic polymer materials suggested their substitution for the organic component(s) in the Corning Glassblend materials as a logical route to improve the high temperature stability of the composite. Since a number of phosphazene based polymers exhibit both water stability and solubility, a new processing route for deriving intimate mixtures of the polymer and glass precursors on a molecular level became evident. Following solvent removal, subsequent chemical reaction between the constituents was expected to promote strong interfacial bonding and evolution of a new insoluble material with enhanced chemical, thermal, and mechanical stability.

Based upon results obtained in the BES funded program, Microstructural Modification in Ceramic Processing Using Inorganic Polymer Dispersants, several phosphazene polymers, $-\left[\mathrm{N}=\mathrm{PR}_{2}\right]_{\mathrm{n}^{-}}$, and cyclic polymer precursors $(n=3,4)$ have been identified as likely candidates for preparing glasspolymer composite materials. The selection was based upon the chemical reactivity of the $-R$ functional group of the polymer. Electronegative groups including secondary and tertiary amines, carboxylic acids, and alkoxides were chosen on the basis of their ability to bind strongly to oxide surfaces in aqueous media. Oxide glasses based upon the phosphate glass-forming system were chosen in analogy to the Corning glass precursor. Since mixed alkali glasses are readily prepared having compositions from the meta- to pyrophosphate system, and since such glasses are both water soluble and water stable from hours to days depending on composition, an aqueous route to the preparation of glass-polymer composites was deemed feasible.

During the past 2 months since this LDRD program started, research has focused upon preparing target composite materials using an aqueous processing route and characterizing the resulting thermally processed material by means of molecular spectroscopy and calorimetry.

Activities involved synthesis of precursor species and spectroscopic characterization using ${ }^{31} \mathrm{P}$ nuclear magnetic resonance, and laser and Raman spectroscopy. Optimization of processing conditions involved selecting appropriate quantities of the precursors to incorporate into the composite and developing a protocol for synthesizing the new material. Following thermal treatment, materials were analyzed to demonstrate that indeed mixing at the molecular level was present and that chemical reactions at the molecular interface between the glass and polymer chains had occurred.

\section{Materials Synthesis}

Phosphate Glass Precursors. Glass compositions ranging from metal oxide-metaphosphate $\left(\mathrm{M}_{2} \mathrm{O} \cdot \mathrm{P}_{2} \mathrm{O}_{5}\right)$ to metal oxide-pyrophosphate $\left(2 \mathrm{M}_{2} \mathrm{O} \cdot \mathrm{P}_{2} \mathrm{O}_{5}\right)$ have been prepared where the metal $\mathrm{M}^{+1}$ represents a single cation or mixture of alkali metal cations. The ammonium $\left(\mathrm{NH}_{4}{ }^{+}\right)$analogues of these glasses also were prepared. Zinc oxide could be incorporated into the linear phosphate chains to increase the flexibility 
of the chains. Raman spectra confirmed the target glass compositions, and cross polarized magic angle spinning (CP MAS) ${ }^{31} \mathrm{P}$ nuclear magnetic resonance measurements indicated no evidence for phase separation. All glasses were water soluble, and the anionic phosphate chains hydrolyzed only after a period of many hour.

Polymer Precursors. In order to test the reactivity of phosphazenes and phosphate glasses, phosphazene trimer and tetramer derivatives which are significantly simpler to prepare than the corresponding polymers were prepared. It is known that these species exhibit chemical reactivity similar to that observed for the polymer analogs. Substituted phosphazene trimers, tetramers, and polymers containing monomethylamine, dimethylamine, and alkoxy moieties were prepared and characterized by means of ${ }^{31} \mathbf{P}$ nuclear magnetic resonance. Water-soluble organic polymers (polyvinyl alcohol, and polymethyl acrylic acid) also were a segment of the test matrix.

\section{Composite Preparation}

Polymer and glass precursors were dissolved in water, then combined at various mixing ratios followed by subsequent heating to remove the solvent and induce reactions between the components. The resulting materials were characterized using CP MAS ${ }^{31}$ nuclear magnetic resonance and laser Raman spectroscopy.

\section{Analysis of New Molecular Composites}

Several molecular composites were formed and ranged from clear transparent materials to opaque samples. In a number of instances direct chemical reaction between the glass and polymer phases was identified in measured Raman spectra. For example, in heattreated mixtures of the monomethylamine tetramer and an ammonium metaphosphate glass, the O-P-O symmetric stretching vibration shifted to lower frequency by $5 \mathrm{~cm}^{-1}$ signifying an opening of the O-P-O angle. An ammonium bending vibration at $900 \mathrm{~cm}^{-1}$ disappeared in the composite signifying loss of ammonia from the final product. Based upon the vibrational data, strong interaction between the anionic phosphate site and the amine site on the polymer has occurred.
Similar changes in the vibrational spectrum have been identified in many of the composites produced using this aqueous route. Differential scanning calorimetry was used to measure the glass transition and decomposition temperatures for a number of prepared samples. In measurements to date, a single endotherm has been observed again confirming that the material is not phase separated. Based upon these studies, mixing on the molecular level and chemical reactivity between the glass and polymer precursors has been confirmed.

\section{Future Work}

Phosphazene polymer precursors stable to higher temperatures vill be used to develop more thermally resilient molecular composites. Candidate materials include sulfate and phosphate derivitized phosphazenes. A single endotherm observed in differential scanning calorimetry measurements of this composites provides evidence for a single phase pure material. If phase separation has occurred, at least two resolved endotherms are expected. However, the resolution of differential scanning calorimetry may not be sufficient to distinguish between two closely spaced features particularly when the precursors have very similar thermal properties. A higher resolution technique based on nuclear magnetic resonance $T_{2}$ measurements can now be performed on the new solids nuclear magnetic resonance facility recently installed in the materials department. Studies using the new equipment on Corning supplied materials as well as PNL derived materials will further help to characterize these new materials. Results will be used to refine processing conditions.

\section{Other Accomplishments}

Work presented in this area at the Materials Research Society and American Ceramic Society sponsored meetings has stimulated interactions with staff at Corning Glass. Drs. Candace Jo Quinn and Charlene Smith are interested in using our solids nuclear magnetic resonance facility to analyze some of their materials. Based upon the results of these initial studies, Corning and PNL likely will propose collaborative work in the glass-polymer composites ares. The solution route developed at PNL offers several advantages over the extrusion process developed at Corning. The possible development of protective coatings is a related area of great interest. 


\title{
Development of a Selective Solid TRU Extractant
}

\author{
B. M. Rapko (Chemical Technology)
}

Currently, the baseline technology in the U.S. for removal of transuranic elements (TRUs) is the TRUEX (transuranium extraction) process. A CMPO (carbamoyl-methylphosphine oxide) is used as the extractant in the TRUEX process, which is a liquid-liquid extraction system for the selective removal of transuranic elements from acidic, aqueous radioactive waste streams. This project involved modifying the compound's structure with the final goal of incorporating the extractant through direct, covalent, carbon-carbon bonding into a polymer framework.

This project naturally divided into several sections. The first (and perhaps most time and resource intensive) section was to synthesize and characterize the polymer precursors, as well as an additional series of model compounds. These model compounds were needed to assess the impact of structural modifications, necessary to covalently incorporate the extractant into a polymer, with the modified compound's ability to extract transuranic elements. The second section was to evaluate the modified compound's ability to extract transuranic elements as a function of nitric acid concentration. The third section was to prepare and characterize polymers containing the transuranic elements extractant. The fourth and final section was to evaluate the polymer's ability to extract transuranic elements in a solid-liquid system with the model extractants in a liquid-liquid system.

The first section has been finished. A list of the compounds prepared according to the procedure as described in the research proposal is shown in Figure 1. The compounds have been characterized by ${ }^{1} \mathrm{H},{ }^{13} \mathrm{C}$, and ${ }^{31} \mathrm{P}$ nuclear magnetic resonance spectroscopy, infrared spectroscopy, thin-layer chromatography, and gas chromatography/mass spectrometry. These compounds are readily prepared in good yield $(>80 \%)$ and with good ( $>95 \%$, usually $>98 \%$ ) purity; the remaining material is unreacted starting compound, which will not interfere with any subsequent polymer chemistry.
The second section has also been completed. Solutions of the four model compounds (i.e., those without a $\mathrm{C}=\mathrm{C}$ double bond) were prepared. These solutions, $0.5 \mathrm{M}$ in extractant in t-butyl benzene, were compared with each other and with the unmodified extractants for their ability to extract $\mathrm{Am}^{3+}, \mathrm{Pu}^{4+}, \mathrm{UO}_{2}{ }^{2+}$, and nitric acid. The results indicate that the size and type of the R group has little effect on the extractant's basicity (approximated by the distribution value for nitric acid) but that the size has a profound effect on the ability of the extractant to remove transuranic eiements from aqueous, acidic nitrate solutions. Figure 2 illustrates the changes observed for one metal ion, $\mathrm{Am}^{3+}$, with one class of modified transuranic element extractant, carbamoyl-methylphosphine oxide, as a function of aqueous nitrate concentration.

These results have serious implications with respect to the behavior of a extractant-containing polymer. These observed steric effects indicate the need for a long spacer group located between the polymer backbone and the extractant functionality in order that the backbone not "bang into" the metal upon complexation and correspondingly reduce its ability to extract transuranic elements from aqueous nitrate solutions. This survey suggests that the 1-octene modified carbamoyl-methylphosphine oxide should be the optimum precursor for the transuranic element-extracting polymer, and provides a focus for subsequent polymer studies.

A complete evaluation of the carbamoylmethylphosphine oxide-based model compounds was complicated by their potent ability to extract $\mathrm{UO}_{2}{ }^{2+}$ and $\mathrm{Pu}^{4+}$, which required reducing the extractant concentration in an attempt to obtain more accurate distribution values. In part because each change in extractant concentration and concomitant transuranic element measurements added weeks to the project's schedule, sections 3 and 4 remain uncompleted.

To summarize, the synthesis and characterization of these modified transuranic element extractants was completed. Their ability to extract transuranic elements such as $\mathrm{Am}^{3+}, \mathrm{Pu}^{4+}$, and $\mathrm{UO}_{2}{ }^{2+}$ from 
aqueous nitrate solutions in a liquid-liquid extraction system have been evaluated. These accomplishments are expected to be submitted for publication during FY 1994. All of the work to date indicates that the concept of designing a solid transuranic element extractant remains a viable one. The actual synthesis, characterizatinn, and testing of such a solid transuranic element-extracting polymer will require additional funding and effort during FY 1994.

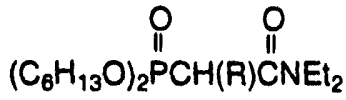

$$
\begin{aligned}
& \mathrm{R}=\mathrm{CH}_{2} \mathrm{CH}\left(\mathrm{CH}_{3}\right)_{2} \quad \text { (Isobutyl) } \\
& \mathrm{C}_{8} \mathrm{H}_{17} \quad \text { (Octyl) } \\
& \left(\mathrm{CH}_{2}\right)_{6} \mathrm{CH}=\mathrm{CH}_{2} \quad \text { (1-octene) }
\end{aligned}
$$

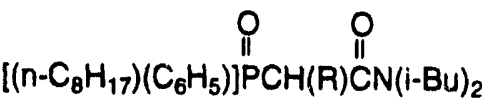

$$
\begin{aligned}
& \mathrm{CH}_{2} \mathrm{CH}\left(\mathrm{CH}_{3}\right)_{2} \text { (Isobutyl) } \\
& \mathrm{C}_{8} \mathrm{H}_{17} \quad \text { (Octyl) } \\
& \mathrm{CH}_{2} \mathrm{CH}=\mathrm{CH}_{2} \quad \text { (Allyl) } \\
& \left.\left(\mathrm{CH}_{2}\right)_{6} \mathrm{CH}=\mathrm{CH}_{2} \quad \text { (1-octene }\right) \\
& \mathrm{CH}_{2} \mathrm{CH}=\mathrm{CH}_{2} \quad \text { (Allyl) }
\end{aligned}
$$

Figure 1. Prepared and Characterized Compounds to Date

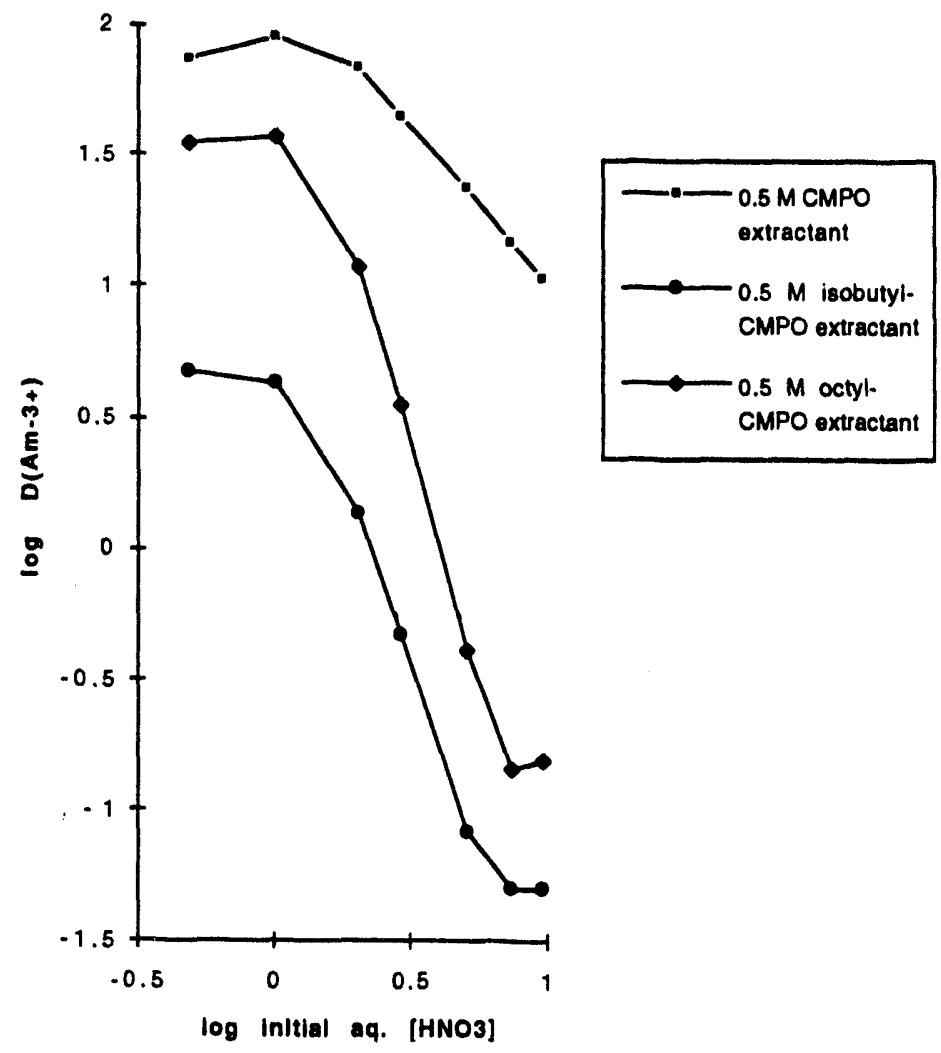

Figure 2. D(Am) Versus [HNO3] - CMPO Extractants 


\section{In Situ Composites Using Tape Casting}

T. R. Armstrung and C. H. Henager (Materials Sciences)

Hot-pressing, the current method of fabricating composites, is not particularly suited to scaling up, nor does it give the control over the starting materials required to control the microstructure and properties of the final product. In this project, tape casting and calendaring fabrication methods were used to produce near-net-shape selfwoven $\mathrm{MoSi}_{2} / \mathrm{SiC}$ composites using $\mathrm{Mo}_{2} \mathrm{C} / \mathrm{Si}$ displacement reactions.

\section{Tape Fabrication}

Silicon and $\mathrm{Mo}_{2} \mathrm{C}$ powders were loaded into polymer, polyvinyl butyral, plasticized with butyl benzyl phthalate using a melt-mixing technique. The solids loading was optimized at $60 \mathrm{vol} \%$. The loaded polymers were rolled into thin tapes by a high shear process, tape calendaring. These tapes were stacked and further consolidated by calendaring to form multilayer specimens.

Calculations were carried out to determine the ratio of $\mathrm{Mo}_{2} \mathrm{C}$ and $\mathrm{Si}$ needed for the reaction to proceed to form $\mathrm{MoSi}_{2}$ and $\mathrm{SiC}$. Based on calculations, the reaction dictates that there be five times more $\mathrm{Si}$ in the starting materials. Based on the density and volume of $\mathrm{Si}$ and $\mathrm{Mo}_{2} \mathrm{C}$, the ratio of tape thickness of $\mathrm{Si}$ to $\mathrm{Mo}_{2} \mathrm{C}$ was determined to be 2.7:1.

A series of tapes were fabricated in which the layer thickness and number of layers were varied so that samples could be generated for a parametric hotpressing study. The $\mathrm{MO}_{2} \mathrm{C}$ tapes were fabricated with layer thicknesses in the range of 7 to $50 \mu \mathrm{m}$ and silicon tapes with thicknesses from 18 to $133 \mu \mathrm{m}$.

The number of layers in the samples was controlled, and was typically 12 to 48 layers each of $\mathrm{Si}$ and $\mathrm{Mo}_{2} \mathrm{C}$.

\section{Binder Removal}

Initially the samples were debindered in air, however, at $400^{\circ} \mathrm{C}$ it was observed that the samples underwent a significant volumetric expansion. This volumetric expansion was associated with the Si layers and presumed to be a result of the oxidation of silicon. However, no associated weight gain was observed when samples were examined by thermal gravametric analysis in air. This problem with specimen expansion was avoided by debindering samples under flowing nitrogen. Samples debindered in air and $\mathrm{N}_{2}$ were hot pressed to access the effect air and nitrogen may have on the kinetics of $\mathrm{MoSi}_{2}$ and $\mathrm{SiC}$ formation.

\section{Sample Densification}

Figure 1 shows a sample hot pressed at $1400^{\circ} \mathrm{C}$ for 4 hours and Figure 2 shows a typical $\mathrm{Si} / \mathrm{Mo}_{2} \mathrm{C}$ interface showing formation of an intermediate phase $\mathrm{Mo}_{5} \mathrm{Si}_{3} \mathrm{C}, \mathrm{MoSi}_{2}$, and $\mathrm{SiC}$. The $\mathrm{SiC}$ platelets showed preferred orientation, $90^{\circ}$ to the interfaces, as predicted from hot-pressed diffusion couples of $\mathrm{Mo}_{2} \mathrm{C}$ and $\mathrm{Si}$.

The particle size of the silicon starting material was large $(>20 \mu \mathrm{m})$. In some instances, when the silicon layer thickness was less than $20 \mu \mathrm{m}$, especially after binder removal, the $\mathrm{Si}$ particles penetrated through the $\mathrm{Mo}_{2} \mathrm{C}$ layers. This was a result of the Si particle size being greater than $20 \mu \mathrm{m}$.

\section{Conclusions}

Multilayer samples were successfully fabricated and densified having distinct layers when the Si layer thickness was greater than $20 \mu \mathrm{m}$. This was due to the Si particle size being greater than $20 \mu \mathrm{m}$. Samples debindered under nitrogen and hot-pressed had ideal microstructures showing preferred orientation of the SiC phase.

\section{Other Accomplishments}

M. Murphy (B.S. in Ceramic Engineering) was supported as a NORCUS student for the course of this project. 


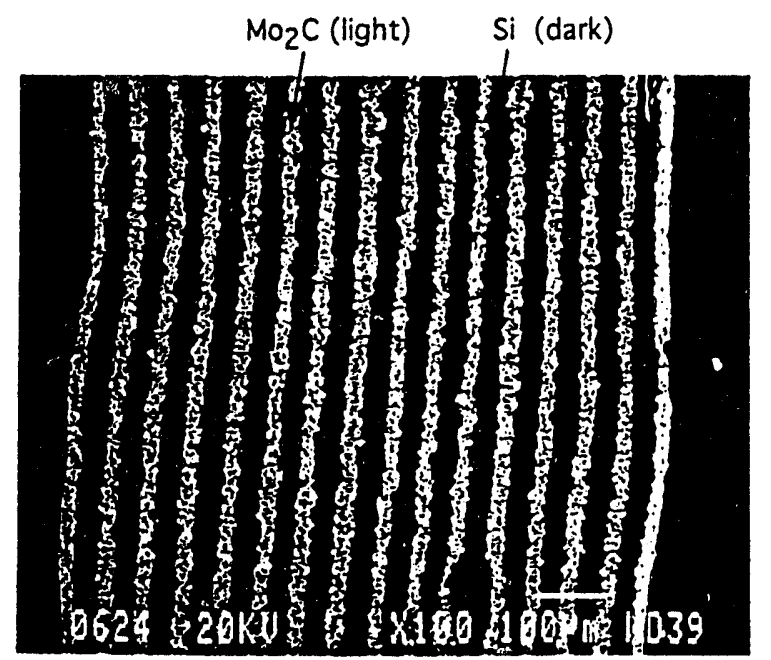

Figure 1. Cross-section of Hot-Pressed $\mathrm{Si} / \mathrm{Mo}_{2} \mathrm{C}$ Composite Showing 16 Distinct Layers of $\mathrm{Si}$ and $\mathrm{Mo}_{2} \mathrm{C}$ Densified for 4 Hours at $1400^{\circ} \mathrm{C}$

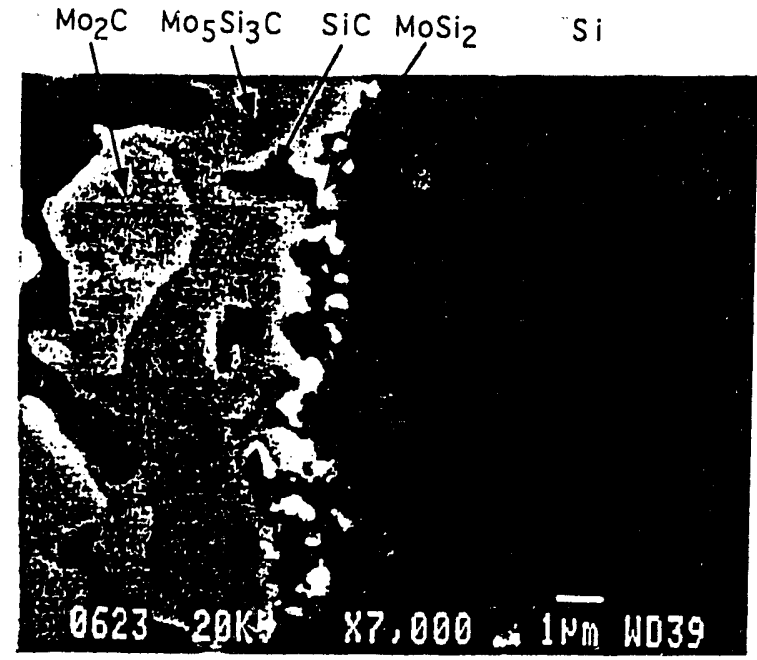

Figure 2. $\mathrm{Si} / \mathrm{Mo}_{2} \mathrm{C}$ Interface Showing Formation of an Intermediate $\mathrm{Phase} \mathrm{Mo}_{5} \mathrm{Si}_{3} \mathrm{C}, \mathrm{MoSi}_{2}$ and $\mathrm{SiC}$ After 4 Hours at $1400^{\circ} \mathrm{C}$ 


\title{
Novel Electrosynthesis of Organic Electroactive Polymers with Organo-Substituted Cyclic Trimeric Phosphaszenes as a Dopant
}

\author{
M. A. Josowicz (Materials Science)
}

The electrochemical introduction of inorganicbased macromolecules into a highly cross-linked organic ultrastructure such as polypyrrole or polythiophene reveals development of new polymeric materials. The insertion of the inorganic non-carbon elements into the backbone of totally organic macromolecules should generate a different combination of properties in polymers than that which are known for totally organic or inorganic materials, exclusively. These polymers are expected to provide a basis for many of the new advanced materials with higher resistance to thermal or oxygen degradation. These materials could also have desirable electronic and ionic characteristics that would make them suitable as protective coatings, solid electrolytes, or novel materials for microelectronic applications.

The goal of this project was to investigate the possibility to electrochemically polymerize materials containing inorganic and organic macromolecules. During this LDRD project period, in situ electrochemical polymerization of cyclic hexachlorophosphazene with p-benzoquinone or hydroquinone was accomplished. The polymerization route provides an easy and reproducible access to formation of a variety of composite polymers directly on electrically conducting substrates. These new composite materials are expected to exhibit high mechanical and temperature stability and high chemical resistivity toward organic and inorganic solvents. The new polymers may find applications as novel materials for the automotive industry, as corrosion protective coatings or in electronic and electroanalytical components such as chemical sensors.

\section{Design of New Electrochemical Routes for Copolymerization of Cyclic Chlorophosphazene Monomers with Organic Electroactive Species}

It is known that the cyclic chlorophosphazene $\left(\mathrm{NPCl}_{2}\right)_{3}$ trimers are not electrochemically active within $\pm 2.0 \mathrm{~V}$ in acetonitrile (Ac) as a solvent and tetrabutylammonium tetrafluoroborate $\left(\mathrm{Bu}_{4} \mathrm{NBF}_{4}\right)$ as the supporting electrolyte. This observation is in agreement with the previous attempts to reduce a number of different cyclo- and polyphosphazenes in the 0 to $-3.0 \mathrm{~V}$ potential range in dimethylformamide with the use of tetraalkylammonium halides as the supporting electrolyte, at mercury electrode (Allcock 1972). It was, therefore, necessary to devise some other approach to electrochemically activate the cyclic phosphazene monomer.

The reductive coupling of the electrochemically generated reactive species from p-benzoquinone $(B Q)$ or p-hydroquinone $\left(\mathrm{H}_{2} \mathrm{Q}\right)$ with the $\left(\mathrm{NPCl}_{2}\right)_{3}$ trimer has been accomplished using a three-electrode arrangement. The cell was equipped with a Pt or $\mathrm{Au}$ working electrode, a Pt foil counter electrode and a $\mathrm{Ag} / \mathrm{Ag}^{+}$- reference electrode. The electroreduction of p-benzoquinone (followed by protonation) or the electrooxidation of $\mathrm{H}_{2} \mathrm{Q}$ (deprotonation) was done in oxygen-free electrolyte. The cyclic voltammograms (CVs) indicate that in both cases an intermediate species is the anion radical of benzoquinone, BQ-. In these experiments the ratio of the peak currents $\left(i_{p a} / i_{p o}\right)$ of the well developed redox waves for p-benzoquinone or $\mathrm{H}_{2} \mathrm{Q}$ is always unity. However, the peak separation potential, $\Delta \mathrm{E}_{\mathrm{p}}=\mathrm{E}_{\mathrm{p}, \mathrm{a}}-\mathrm{E}_{\mathrm{p}, \mathrm{c}}=$ $140 \mathrm{mV}$, indicates that the overall process involves an inserted chemical step. This observation agrees well with the generally recognized electrochemicalchemical-electrochemical (ECE) reaction mechanism 
(Pyun and Park 1985). In such cases, the rate of the chemical reactions determines the peak height of the reoxidation peak for the anion radical.

The recorded cyclic voltammograms for the reduction of p-benzoquinone in the presence of the $\left(\mathrm{NPCl}_{2}\right)_{3}$ trimer (PHz) are different. In the presence of the $\mathrm{PHz}$ a fraction of the electrochemically generated BQ- is consumed by the coupling reaction and is no longer available for reoxidation to p-benzoquinone. As a result, the formal electrode potential for $B Q-/ B Q$ is shifted to a more negative potential. As the polymer film formation progresses on the electrode, the formal electrode potential slowly reaches the same peak separation potential. In the vicinity of the cathode the local $\mathrm{pH}$ increases due to the formation of electrogenerated base (EGB). Thus, the conditions for dehalogenation of the $\left(\mathrm{NPCl}_{2}\right)_{3}$ are provided. The BQ- species reacts with the phosphorus-chlorine bonds of the organic phosphazene monomer. The electrochemical experiments also provide some information about the termination factors of the polymerization reaction.

\section{Characterization of the Physical and Chemical Properties of the New Polymer Coating}

The composition and stoichiometry of the inorganic/ organic polymer composite coating was examined by $\mathrm{x}$-ray photoelectron spectroscopy and Raman and Fourier transform infrared spectroscopy. From the $\mathrm{X}$-ray photoelectron spectroscopy data it was possible to determine the at nic composition and stoichiometry of the electrochemically polymerized films. The composition in relative atomic percent normalized to phosphorous is: $\mathbf{F}: \mathbf{O}: \mathbf{N}: \mathbf{C}: \mathbf{C l}: \mathbf{P}: \mathbf{B}=0.24$ :1.55:1.09: $4.43: 0.96: 1.00: 0.62$ (atom \%). The Auger lines of $\mathrm{P}$ and $\mathrm{N}$ are closer to $\left[\mathrm{NP}(\mathrm{OPh})_{2}\right]_{3}$ than they would be in a linear phosphazene polymer. If the aromatic character of the composite material can be assumed, the results would suggest that half of the
$\mathrm{Cl}$ atoms have been substituted for the $\mathrm{BQ}-$ anion. The presence of the boron and fluorine atoms in the electrochemically synthesized film suggests that the inorganic/organic conjugated polymer can exist in a variety of structures due to the presence of radical cations. The presence of the counteranion, $\mathrm{BF}_{4}^{-}$ results from the condition of phase electroneutrality. It leads to so-called "self-doping" of the polymer.

Raman and multiple-attenuated total reflection Fourier transform infrared spectroscopy have been used for polymer characterization of the chemical bonding. The polymer samples were analyzed as deposited on glass/Ti,W/ Pt or glass/Ti,W/ Au substrates. A white, colorless polymer film suggests a short penetration depth (determined by a number of factors, including angle of incidence of the light and the refractive index). In contrast to the weak lines obtained in the Raman spectra, the Fourier transform infrared spectra provide a possibility to characterize the polymer film. The recorded spectra show multicomponent bands. In those spectra, the species of the staring component for the polymerization of the composite material can be assigned. A detailed interpretation of the Fourier transform infrared spectra is being performed.

The films exhibit a regular type morphology: Thicker films $(\sim 5$ to $10 \mu \mathrm{m})$ are rough and electrically insulating. The differential scanning calorimetry (DSC) performed using a heating rate of $10^{\circ} \mathrm{C} / \mathrm{min}$ in nitrogen shows a transition around $300^{\circ} \mathrm{C}$. The $x$-ray diffraction experiments indicate an amorphous film structure.

\section{References}

Allcock, H. R. 1972. "Phosphorous-Nitrogen Compounds." Academic Press.

Pyun, C., and S. Park. 1985. J. Electrochem. Soc. 132:2426. 


\subsection{National Security and Defense}




\section{CW Molecular Discriminator}

R. C. Hanlen (National Security and Defense)

Multivariate statistical approaches will be developed to describe and discriminate the generic molecular structure and characteristics of chemical weapons (CW) agents from other similar chemical structures (i.e., pesticides, herbicides, and fungicides).

This project was started late in the fiscal year (September 1993). A brief outline of data requirements was made and some data were acquired/ retrieved using PNL's technical library staff. The data that was retrieved from Dialog, an online vendor service for scientific and technical literature and information, pertains to the chemical properties associated with pesticides, herbicides, fungicides, and some chemical weapons agents.

The technical progress expected during FY 1994 consists of completion of data gathering and the development of prototype molecular structure discriminants. 


\title{
Environmental Sampling Strategies
}

\author{
G. R. Holdren (Geosciences)
}

The objective of this study was to examine the potential benefit of incorporating an environmental sampling strategies component into nuclear nonproliferation verification activities.

With the changing political situation in the world, compliance with nuclear nonproliferation treaties is becoming viewed as increasingly critical for stable international relations. Multiple approaches can be undertaken to verify compliance with these agreements. Sampling and evaluation of environmental media for the byproducts and wastes generated during the processing of nuclear materials is a potentially effective method for determining the nature and extent of activities being undertaken at a site or in a geographic region.

Production and processing of special nuclear materials generates waste streams containing distinct signature materials including radiochemical, base metal, and organic compounds. During the normal course of operations and in the absence of substantial technological efforts, these signature materials are released to the environment and dispersed downwind or downstream from the source. Once in the environment, it is virtually impossible to conceal or disguise the presence of these materials until their concentrations become diluted to levels approximately equal to background. For this reason, an environmental sampling activity run in conjunction with broader surveillance or monitoring programs can be used to corroborate information obtained regarding known facilities, and to provide prima facia evidence regarding the potential existence of undeclared facilities. In addition, the existence of such a strategy could, potentially, act as a deterrent, since the threat of detection might be sufficient to discourage countries from initiating undeclared activities.
At this point, substantial information is known about disparate parts of the system: what the critical signature materials are, what processes generate which signatures, analytical techniques for detecting signatures in a variety of media, and natural concentrators that serve as effective and efficient traps for collecting the signatures in distinct environments. However, linkages among these various aspects of the problem have not been fully developed. Substantial increases in the effectiveness of field sampling programs to detect proliferating activities would be realized if these various pieces of information were assembled and correlated in a systematic way.

The goal for this project was to initiate an effort to determine what linkages are needed to develop an effective sampling strategy, and to identify a project team capable of providing guidance on major technical issues that develop during the course of the work.

\section{Critical Linkages}

The first step in developing a sampling framework is to identify the major components of the system to be studied, and then to identify critical path linkages between the different components in the system.

Figure 1 illustrates the major types of information that will be required to develop a sampling framework. At this point, we have also assembled a project team to identify and pursue technical issues that become apparent as we explore the problem and attempt to develop a formalized sampling framework.

This project was initiated late in the fiscal year, and had minimal expenditures. The work is being continued under funding from the Advanced Concepts Program. 


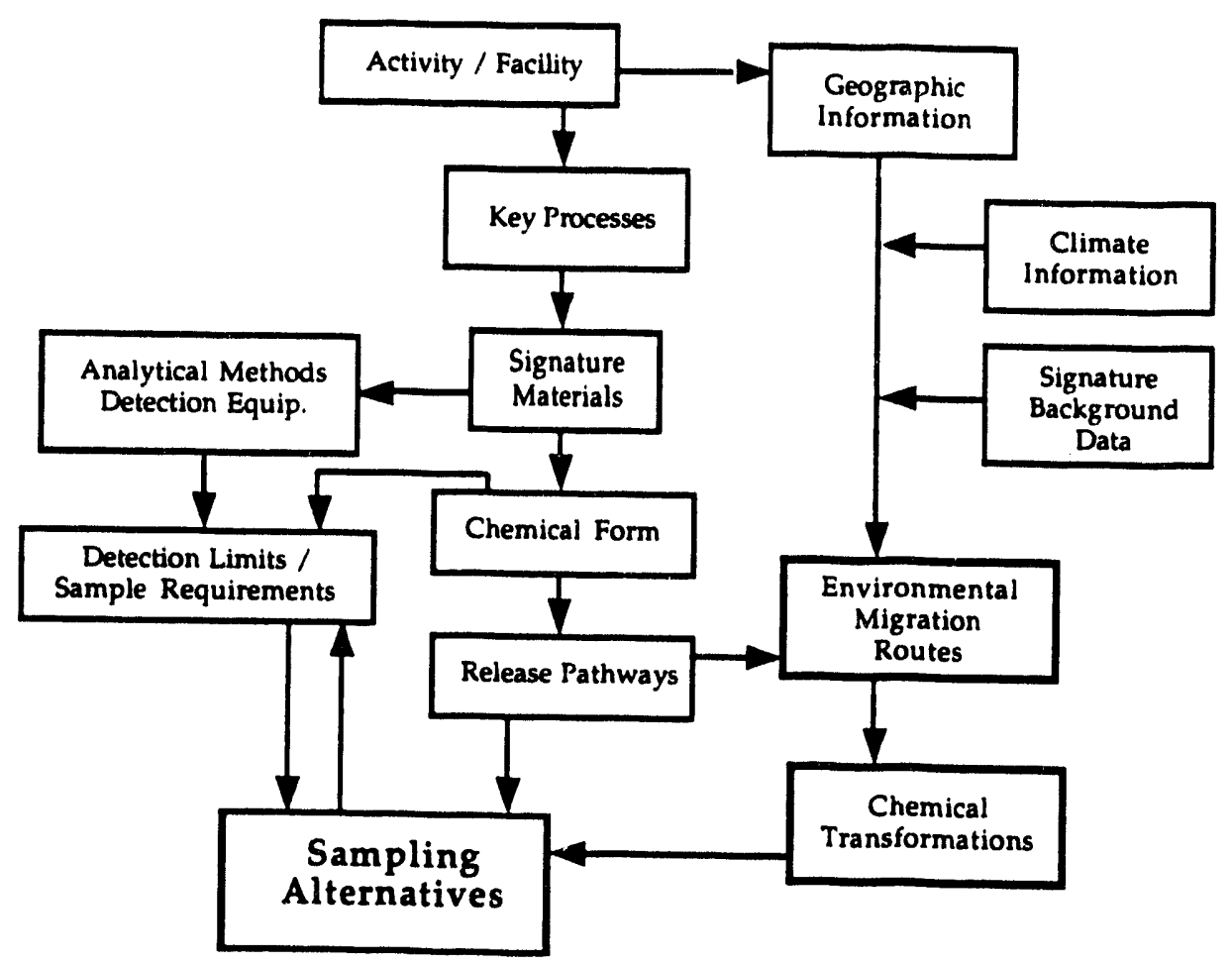

Figure 1. Diagram of the Major Components to be Taken into Consideration in Developing ampling Strategy to Detect Noncompliance Nonproliferation Agreements 


\title{
Material Structural Properties and Physics of Scintillators
}

\author{
M. Bliss (Chemical Sciences)
}

The objective of this project was to develop an understanding of the electronic relaxation process in scintillating materials by examining the relationships between material structure, electronic relaxation processes, and light output within a group of structurally and chemically similar materials.

During FY 1992, the behavior of scintillation light was studied for a series of cerium-doped alkali silicate glasses. Measurements performed included light output, spectral content, and spectrally resolved fluorescence lifetime. Measurements were made as a function of alkali, alumina, magnesia, and ceria concentration and of annealing history. In this system, the cerium serves as a sensitive probe of the local glass structure. Data collected supported the conceptual model that the nonbridging oxygens serve as the primary trap for the excitations that represent ionization. However, additional data showed that the situation is more complicated than that described by this simple model. In addition, some theoretical work relating to the first stage of the transfer of energy from the ionizing radiation to the solid was performed; these calculations provided new insight into this part of the scintillation process.

In FY 1993, the primary thrust was to develop technical information on the results of FY 1992 activities for the scientific community. In addition, low temperature thermoluminescence and Raman measurements were performed on a lithium aluminosilicate composition series. Dissemination of FY 1992 and
FY 1993 results were achieved by presentation at professional society meetings, international conferences, preparation of scientific papers for the refereed literature, and preparation of a summary report of FY 1992 project activities.

\section{Publications}

Bliss, M., R. A. Craig, and P. L. Reeder. "Method for Measuring the Light Output of Scintillating Glass Shards." Nucl. Inst. and Meth., submitted.

Bliss, M., R. A. Craig, and P. L. Reeder. "The Physics and Structure-Property Relationships of Scintillator Materials: Effect of Thermal History and Chemistry on the Light Output of Scintillating Glasses." Nucl. Inst. and Meth., submitted.

\section{Presentations}

Bliss, M. 1993. "Low-Temperature Thernoluminescent Investigations of Trap Distributions in Scintillating Glasses." Presented at the International Conference on Luminescence, Storrs, Connecticut.

Bliss, M., R. A. Craig, and P. L. Reeder. 1993. "The Effects of Thermal History and Structure on Light Output of Scintillating Glasses." Presented at the March 1993 Meeting of the American Physical Society, Seattle, Washington.

Craig, R. A., M. Bliss, and P. L. Reeder. 1993. "Energy Transfer of lonizing Radiation Into Light Emitting Dielectric Materials." Presented at the March 1993 meeting of the American Physical Society, Seattle, Washington.

Reeder, P. L., M. Bliss, R. A. Craig, K. Stahl, and M. J. Weber. 1993. "Spectrally Resolved Fluorescence Decay Lifetimes for Scintillators." Presented at the March 1993 Meeting of the American Physical Society, Seattle, Washington. 


\subsection{Reactor Technology}




\title{
Ceramic and Metal Matrix Composites in Nuclear Reactor Applications: A Preliminary Study
}

\author{
D. J. Senor (Nuclear Systems and Materials)
}

\begin{abstract}
Composites were initially introduced into aerospace applications because of their high specific strength and stiffness. Outside the aerospace industry, composites have been restricted to limited applications due to their high cost compared to monolithic materials. However, it is possible to identify applications in highly specialized industries for which the performance gains offered by composites outweigh the increased production cost. The nuclear industry is an example of such an area.
\end{abstract}

The primary advantage of composites is that they may be tailored to specific design needs by varying the matrix and reinforcement material and morphology. There has been limited research into potential applications for composites in nuclear applications, but the adaptability of these materials creates possibilities for their use in virtually all aspects of the nuclear industry. Table 1 presents several materials limiting issues relevant to nuclear applications in which composites may exhibit an advantage over traditional monolithic materials.

This study reviewed previous work related to the use of composite materials in the nuclear industry. Based on these experiences, and coupled with existing needs, the most promising future applications for composites in the nuclear industry were identified. Near-term actions were identified, which are necessary to initiate a coherent program at PNL devoted to the development of composites for nuclear applications. These recommendations were based on both technical and programmatic issues.

\section{Literature Review}

A literature review was conducted to identify all actual or proposed uses of composites in the nuclear industry. Included in the review were commercial light water reactors (LWRs), advanced terrestrial reactors such as liquid metal reactors (LMRs) and gascooled reactors (GCRs), as well as fusion reactors, space reactors, radioisotope thermoelectric generators (RTGs), and high-level radioactive waste disposal issues.

\section{Promising Applications for Composites}

Fusion and space reactor applications were judged to be the most appropriate for the introduction of composite materials due to the lack of monolithic materials adequate to perform in these environments. Composites are appropriate for selected terrestrial fission reactor applications, but the net performance gain achieved by introducing these materials would be marginal at best, due to the high cost of the materials and the strict regulatory environment associated with the commercial power reactor industry. Nuclear waste storage and encapsulation applications were not explored due to the inability of composites to offer a unique advantage over materials currently in use.

Monolithic vanadium alloys have recently been selected as the most likely candidates for first wall and blanket structural materials for the International Thermonuclear Experimental Reactor (ITER). Pure vanadium metal is ideal for fusion applications since it does not activate to an appreciable degree, but it also does not possess the required high temperature strength. Therefore, it is necessary to alloy vanadium with minor additions of elements such as $\mathrm{Cr}, \mathrm{Ti}$, or Fe. However, this results in unacceptably high neutron activation, causing maintenance and decommissioning problems. A combination of high temperature strength and low neutron activation may be possible using a composite system such as $\mathrm{SiC} / \mathrm{V}$.

SiC/V composites have not previously been produced, and fabricability would be the primary issue for an initial research program. Vanadium metal processing is similar to that used for titanium. Since SiC/Ti composites have been successfully produced, it is reasonable to expect $\mathrm{SiC} / \mathrm{V}$ to yield similar results. However, thermodynamic analysis of the $\mathrm{SiC} / \mathrm{V}$ system indicates that formation of vanadium carbides at the matrix/reinforcement interface is possible at temperatures as low as $25^{\circ} \mathrm{C}$. Vanadium silicides may 
also form at higher temperatures. These effects may be mitigated by coating SiC-based fibers with BN, but formation of vanadium borides is probable at temperatures above $1000^{\circ} \mathrm{C}$. Coatings such as $\mathrm{BN}$ may also reduce the strengthening benefit of the fibers and may introduce thermal expansion mismatch problems. If interfacial compound formation proves a serious problem with uncoated fibers, a possible alternative to $\mathrm{SiC}$ is an $\mathrm{Al}_{2} \mathrm{O}_{3}$-based fiber.

\section{Recommendation}

The most attractive follow-on experimental activity is a study of the fabricability of composites for fusion reactor structural applications. The SiC/V system should be studied for compatibility, fabricability, and microstructural and material properties characterization. The viability of such a program would be enhanced by cooperative research and development agreements with vendors within the materials industry.

\section{Other Accomplishments}

Dr. D. J. Senor (Ph.D. Nuclear Engineering) was hired from Texas A\&M University immediately prior to the initiation of this project.
Table 1. Relevant Materials Limiting Issues

\begin{tabular}{||l|l||}
\hline $\begin{array}{l}\text { Materials limiting } \\
\text { issues }\end{array}$ & \multicolumn{1}{|c|}{$\begin{array}{c}\text { Potential composite } \\
\text { advantages }\end{array}$} \\
\hline Neutron activation & $\begin{array}{l}\text { Replacement of high activation } \\
\text { metal alloys by low activation } \\
\text { matrix and reinforcement }\end{array}$ \\
\hline $\begin{array}{l}\text { Thermal } \\
\text { conductivity }\end{array}$ & $\begin{array}{l}\text { High conductivity fibers for } \\
\text { directed heat flow; high } \\
\text { conductivity matrix with low } \\
\text { conductivity particles }\end{array}$ \\
\hline $\begin{array}{l}\text { Irradiation/thermal } \\
\text { stability }\end{array}$ & $\begin{array}{l}\text { Dimensionally and chemically } \\
\text { stable matrix with inert } \\
\text { ceramic reinforcement }\end{array}$ \\
\hline Corrosion & $\begin{array}{l}\text { Corrosion resistant metals } \\
\text { reinforced by high strength } \\
\text { fibers }\end{array}$ \\
\hline $\begin{array}{l}\text { High temperature } \\
\text { tensile/creep } \\
\text { strength }\end{array}$ & $\begin{array}{l}\text { Ceramic fibers extend metal } \\
\text { creep resistance to near } \\
\text { melting point }\end{array}$ \\
\hline $\begin{array}{l}\text { Specific } \\
\text { strength/stiffness }\end{array}$ & $\begin{array}{l}\text { Low density and high strength } \\
\text { fibers reduce overall weight }\end{array}$ \\
\hline
\end{tabular}




\title{
Closed Chamber Electrolyte System for Separation of Graphite from Radioactive Materials
}

\author{
G. W. Hollenberg (Nuclear Systems and Materials)
}

The objective of this project was to develop an approach for separation of radioactive species from carbon-based waste forms and for decomposition of hazardous organic waste streams. The method is based upon closed chamber incineration by pure oxygen, with discharge of the reaction products through molten carbonate fuel cells.

Closed chamber incineration (CCI) is a novel technique where radioactive or hazardous carbon-based materials may be destroyed without the risk of potentially dangerous cation releases into the environment. The process utilizes an enclosed combustion chamber coupled with molten carbonate fuel cells (MCFCs). Because only anion transport is allowed by the molten carbonate fuel cells, cation release to the environment is intrinsically suppressed. Oxidation of carbon-based materials may be tailored such that the principal combustion product is $\mathrm{CO}_{2}$. The $\mathrm{CO}_{2}$ is conducted through the molten carbonate fuel cells for release to the environment by the chemical reactions

$$
1 / 2 \mathrm{O}_{2}+\mathrm{CO}_{2}+2 e^{-} \rightarrow \mathrm{CO} / \mathrm{s}^{-}
$$

at the cathode (on the combustion chamber side) and

$$
\mathrm{H}_{2}+\mathrm{CO}^{-} \rightarrow \mathrm{CO}_{2}+\mathrm{H}_{2} \mathrm{O}+2 e^{-}
$$

at the anode (on the effluent side). The process is schematically represented in Figure 1 for a single molten carbonate fuel cell. Due to their preferential transport of carbonate anions, molten carbonate fuel cells are ideal for applications requiring separation of $\mathrm{CO}_{2}$ from potentially hazardous materials.

The most important feature of the molten carbonate fuel cell with respect to the destruction of radioactive or hazardous materials is the electrical potential developed (or imposed) during operation. Cation transport from the cathode to the anode is electrostatically impossible. Thus, the transport of radioactive cations (e.g., strontium, cesium, plutonium, americium, etc.) is intrinsically suppressed and mobile cations carried to the fuel cell will remain within the closed combustion chamber.

Fuel cells were initially developed for electrical power production, but they are increasingly used in many applications requiring elemental or molecular separation. Typically, fuel cells operating as separation devices are operated in a driven mode with an externally imoosed electrical potential difference across the cell. 1. wever, they may also be operated in a power producing mode using a fuel gas as shown in Figure 1.

\section{Applications}

Large graphite moderated reactors have been built in several nations. Many of these reactors are either decommissioned or nearing the end of their service life. These reactors account for more than 1.5 million tons of radioactively-contaminated graphite.

Permanent geologic disposal of this material as highlevel waste is inefficient and costly due to its large volume. Incineration coupled with effluent filtration with and without conventional scrubbers is being considered by several nations. Although scrubbers perform essentially the same role as the molten carbonate fuel cells in this concept, performance concerns have traditionally limited the acceptance of this option in the United States. Closed chamber incineration eliminates these concerns by providing intrinsic cation transport suppression, while separating the high-level waste components from the graphite this reducing the volume of waste for vitrification and geologic disposal.

A detailed examination of existing inventories of nuclear graphite was conducted to produce a specific example which would provide a focus for determining the feasibility of closed chamber incineration. The most critical near-term application for this technology is the disposal of high temperature gas reactor (HTGR) fuel elements. This application represents both a reduction of proliferation concerns, as well as a high-level waste volume reduction. Current disposal 
options for these materials include vitrification and/or geologic disposal. Vitrification would ease the proliferation threat, but requires separation of the fue: particles from the graphite. Past plans have centered around conventional incineration or crushing operations to perform the separation. Both of these options appear unacceptable due to the significant radiological risk of fission product release into the environment.

\section{Fuel Processing}

A preliminary design of a closed chamber incineration assembly that would process individual fuel blocks was developed and is shown schematically in Figure 2. The goal of closed chamber incineration processing is to incinerate the graphite matrix material without requiring any crushing operations in order to separate the ruel kernels. Utilizing a pure oxygen atmosphere and impurity catalysis, complete oxidation of an entire $110 \mathrm{~kg}$ block of graphite should take approximately 2 hours. Carbon dioxide produced from oxidation of the graphite is transported through the molten carbonate fuel cell, reacts with the fuel gas, and is vented. The SiC coating on the fuel kernels is converted to $\mathrm{SiO}_{2}$, and removed after oxidation is complete for transfer to a vitrification facility. A molten carbonate fuel cell stack consisting of 146 fuel cells (approximately $10 \times 10 \times 60 \mathrm{~cm}$ ) is adequate for conducting the incineration of the entire

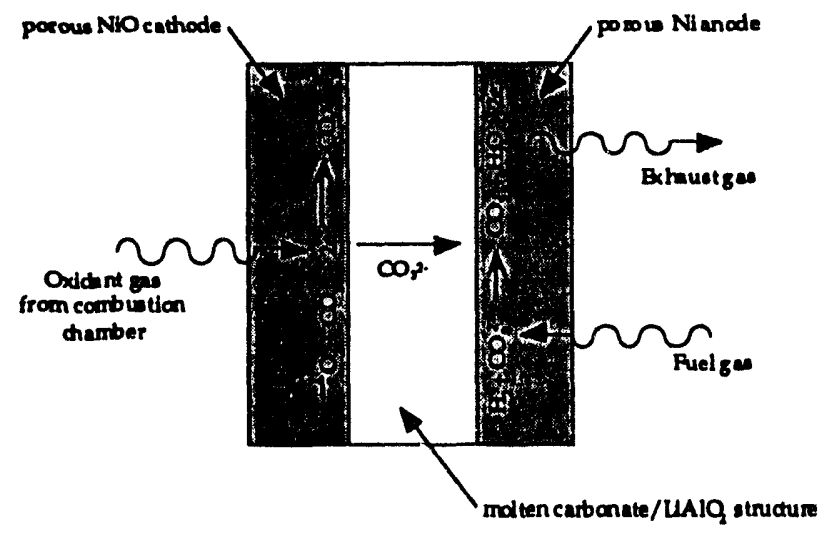

Figure 1. Molten Carbonate Fuel Cell Operation block. The size and operation of a closed chamber incineration unit designed to incinerate fuel blocks from a reactor is compatible with existing hot cells. Oxidation of the fuel blocks provides an $87 \%$ volume reduction, thus facilitating vitrification and disposal of the remaining fuel kernels.

Fuel cell operating parameters were optimized for this incineration process from experimental data compiled at the Institute of Gas Technology (IGT) during development of molten carbonate fuel cells for electrical power production. Calculations and previous experience at the Institute of Gas Technology confirm the anticipated performance of the molten carbonate fuel cells for use in closed chamber incineration.

\section{Publication}

Senor, D. J., G. W. Hollenberg, W. C. Morgan, and L. G. Marianowski. "Destruction of Nuclear Graphite Using Closed Chamber Incineration." In Proceedings of the International Symposium on Extraction and Processing for the Trearment and Minimization of Wastes, TMS, San Francisco, California, February 1994.

\section{Other Accomplishments}

D. J. Senor (Ph.D. Nuclear Engineering) was hired from Texas A\&M University immediately prior to the initiation of this project.

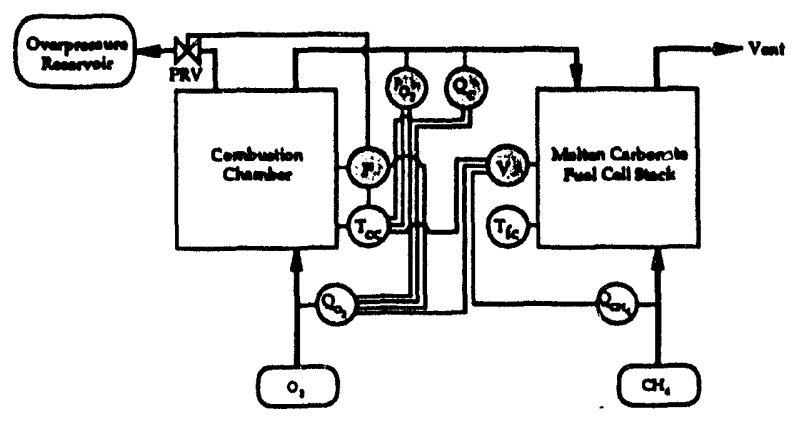

Figure 2. Closed Chamber Incineration System Schematic 


\title{
Establishment of Experimental Capability for Beta Alumina Work
}

\author{
G. W. Hollenberg (Nuclear Systems and Materials)
}

The objective of this project was to establish the capability to operate beta-alumina cells at PNL that are capable of separating sodium from larger and multivalent cations in Hanford waste tank salt cake. Beta-alumina offers a unique capability for rapid ionic conduction of sodium. Ions such as cesium, strontium, uranium, and plutonium are essentially not conducted.

Electrolytic sodium separation from radionuclides is based upon the selectivity and high mobility of sodium ions in a class of ceramics known as sodium fast ion conductors. The original work in this area was performed at the Ford Motor Company in the 1960s during work on the sodium-sulfur battery. It was discovered that diffusion of sodium ions in $\beta$-alumina was up to $10^{5}$ times greater than cesium. In the mid1980s, an National Science Foundation (NSF) program developed and experimentally verified a sodium electrorefining/electrowinning apparatus using $\beta$ "alumina which produced pure sodium directly from impure sodium chloride/aluminum chloride mixtures. Ions larger than sodium or having higher valence states than $\mathrm{Na}^{+}$were not effectively conducted through the $\beta$ "-alumina. Recently, low temperature ceramic sodium ion conductors such as NASICON (sodium silicon conductor) have been developed. These materials offer the potential for electrochemical transport of sodium at ambient temperatures and in aqueous environments. The amount of nonradioactive sodium in the Hanford waste tanks currently dictates the volume of Hanford Waste Vitrification Plant (HWVP) glass necessary for waste disposal. The volume of Hanford Waste Vitrification Plant glass required equates to approximately $10^{6}$ disposal canisters for the existing waste volume. Therefore, initial removal of sodium from either supernate or salt cake (primarily $\mathrm{Na}_{2} \mathrm{NO}_{3}$ ) without the removal of radionuclides constitutes a significant concentration of processing volume. A schematic representation of a $\beta^{\prime \prime}$-alumina tube used for electrorefining salt cake is shown in Figure 1.

\section{Experimental Capability Development}

Extensive sodium research was conducted in the 325 and 326 buidings at Hanford during the 1970s and 1980s while the Fast Flux Test Facility (FFTF) was preparing for operation. Unfortunately, these capabilities have either already been, or are currently being dismantled. In order to conduct electrochemical separation studies with sodium fast ion conductors, an experimental capability was developed in a nonradioactive inert gas glovebox in the 326 Building. The experimental apparatus consists of an insulated $\beta^{\prime}$-alumina tube in a sodium holding tank with appropriate temperature control instrumentation. An electric power supply suitable for sodium electrorefining was installed. Surplus sodium metal was obtained, and nonradioactive cesium metal supplies were identified.

Although funding did not permit the initial operation of the cell, a plan for future experimental activities was developed. Initial studies will focus on separating pure sodium from a melt of sodium metal with approximately $500 \mathrm{ppm}$ of nonradioactive cesium impurities. Analytical composition measurements will be conducted on both the impure sodium starting material and the electrorefined sodium produced during the experiment.

PNL Laboratory Safety evaluated the experimental setup several t wes during development, and modifications were made to accommodate concerns. No outstanding safety issues existed at the conclusion of work for conducting sodium refining in the 326 Building laboratory.

Other Accomplishments

Dr. D. J. Senor (Ph.D. Nuclear Engineering) was hired from Texas A\&M University immediately prior to the initiation of this project. 
$\bar{\lambda}$ 
2.7 Technology Planning and Analysis 


\section{Evaluating the Carcinogenic Risk of Chemical Mixtures}

J. D. Longstreth and R. E. Hegner (Technology Management Planning)

Risk assessments at hazardous waste sites generally must consider the potential adverse health effects of simultaneous exposure to more than one chemical. Current risk assessment guidance for chemical carcinogens is to assume an additive effect of each individual chemical, assuming independence of action by the compounds involved. If, instead, two or more chemicals interact in a synergistic or antagonistic manner, overestimation or underestimation of the actual multiple-substance risk could result. EPA has recently developed computer-assisted techniques for estimating the combined carcinogenic potential of chemical mixtures and is developing a data base which contains estimated combined cancer potency factors for specific mixtures of chemicals. This project was designed to use existing data from Hanford to identify specific mixtures of carcinogens to which workers or other individuals may be exposed, use EPA's expert system and data base to identify high and low potency chemical mixtures present at Hanford, and use existing data on concentrations to identify high and low risk chemical mixtures present at Hanford.
This project started late in FY 1993 (July) when funding became available. An initial evaluation of the most comprehensive data base for Hanford (i.e., the data base for the Programmatic Environmental Impact Statement) revealed very few sources with documented mixtures of chemical carcinogens. Many sources contained mixtures of radionuclides and chemical carcinogens. In addition, EPA's expert system, expected to be available in August 1993, was not released in time to complete this project before the end of FY 1993. Consequently the effort was terminated. 


\title{
Process Re-Engineering Support System (PRESS)
}

\author{
L. O. Levine (Technology Systems Analysis)
}

The objective of this project was to explore the requirements for automated tools to assist process re-engineering teams in discrete manufacturing environments. It explored how expertise in process re-engineering might be inexpensively disseminated to the many small manufacturers in the U.S.

Efforts during FY 1993 focused on two areas:

1. clarifying the target users for these tools (e.g., PRESS)

2. identifying enabling technologies to support effective training and specific process improvement projects.

\section{Target Users}

Contacts with small manufacturers and organizations providing technical support to small manufacturers indicated that the users of tools, such as PRESS, would probably be manufacturing extension organizations, rather than small manufacturers directly. However, the use of PRESS by manufacturing extension organizations as part of their technical outreach services will have to compete with other means of providing technical services outreach (e.g., subsidized use of graduate students in industrial and mechanical engineering). In general, the current scope of such outreach services in the U.S. suggests this idea will have difficulty finding significant use in the next few years.

\section{Enabling Technologies}

Research into technical approaches to provide PRESS indicated that computer-aided intelligent tutoring showed promise as the means to support this concept. However, this technology would probably need to be part of a broader strategy of manual training techniques and group collaboration based on other telecommunication technologies.

\section{Conclusions}

Although PRESS is a promising idea, due to the complexity of implementation and a limited near-term market, the principal investigator recommended that PNL management redirect funding associated with this project to other efforts. 


\title{
Proliferation Analysis Support System
}

\author{
F. L. Greitzer (Technology Systems)
}

This project examined intelligent support concepts for decision makers and analysts in international nuclear safeguards and security. The aim was to define collaborative human-machine interface concepts that can enhance an analyst's access to information, capability to recognize and evaluate the data, and ability to make recommendations. The work complements current efforts to upgrade the information treatment methods of the International Atomic Energy Agency (AAEA).

As a result of experiences in Iraq, the International Atomic Energy Agency (IAEA) is attempting to strengthen safeguards and provide early warning of possible nuclear-related activities that are inconsistent with a state's peaceful-use obligations. The purpose of this research was to explore the application of automation technology to aid the IAEA Country Officer in performing proliferation analysis. The approach was to examine user-system relationships that exploit the capabilities of the human, as well as the machine. The goal is to create a collaborative human-machine system, built upon well-integrated functions at the task level, to yield better overall systim performance. The following broad areas of collaborative support for the IAEA Country Officer were identified:

- portfolio management

- information filtering and retrieval

- analysis

- decision making.

Specific support functions within each of these areas were defined and implemented within a demonstration or concept prototype called Proliferation Analysis Support System (PASS). The interactive Proliferation Analysis Support System prototype runs on a Macintosh computer.

\section{Portfolio Management}

Portfolio management support addresses the administration and maintenance of country files. Information is obtained through a variety of sources, including open source data bases and reporting instruments from the state itself as a signatory of the nuclear nonproliferation treaties. This large body of information must be catalogued and organized so that the Country Officer will be able to access required data. Data includes general information, such as population and geographic features, industrial production statistics, and commerce information on imports or exports. Commercial, off-the-shelf software can be used to help organize various types of information, reports, and critical requirements and information needs. Specialized tools can be developed to provide functions that are not met by commercial office applications.

\section{Information Filtering and Retrieval}

For the IAEA Country Officer, a document and information filtering and retrieval system should support integration and interpietation of information across various sources and over extended periods of time. Key features include

- context-sensitive text analysis

- capability for interactive reorientation

- exploitation of human cognitive abilities through human-machine collaboration

- user training to maximize effective use of the system.

A collaborative approach to text analysis assigns to the computer continuous filtering of new data sources, 
while more context-specific queries may be invoked by the analyst. The process is iterative: When a query result is displayed, the Country Officer may recognize implications by associating information in a novel way and then refocusing the search. This process may produce query topics several layers removed from the initial concepts. Through this interaction, the system can incorporate knowledge of these new relationships into future searches.

\section{Analysis Support}

In conducting their analyses, Country Officers attempt to forecast nuclear material and facilities that will be under safeguards and to estimate production of plutonium and highly enriched uranium at the facilities. Country Officers must recognize patterns of data that point to the existence of clandestine nuclear weapons development activities. Support for analysis requires tools to help recogrize patterns in safeguards data and identify safeguards-relevant features and trends. Two such concepts are a weaponization path capability matrix and a weaponization path flowchart. These displays seek to enhance the analyst's ability to visualize and integrate information from diverse sources through hypertext associative links the analyst creates in the process of information treatment activities.

\section{Decision Making Support}

Because of the extended time periods involved in proliferation analysis and difficulties with incomplete and often misleading information, a key need exists to support information integration (data fusion) and information visualization to help identify non-peaceful use or diversion of nuclear or other technologies for clandestine weaponization. In turn, collaborative human-machine system concepts can help the analyst integrate the emerging evidence into a cohesive picture.

An electronic note card system helps annotate and associate bits of related documents. Using a note card filing system metaphor, the system would provide links back to source documents, as well as forward links to the arguments or cases the Country Officer is developing. Each such case would also be organized in a data base that presents the logical process in a consistent fashion, along with annotations by the user and links back to the note card data base and the original source information. These links would then have links to the weaponization path capability matrix and flowchart displays.

The key collab rative functions comprising this support concept are automated handling of the basic elements-notes representing accumulated evidence over long periods of time, cases representing hypotheses and logical arguments that the Country Officer develops over extended periods, and electronic links among these components. The electronic links serve to document the process.

\section{Conclusions}

Collaborative human-machine interface concepts stress the need for problem-driven system design, as opposed to design that is technology-driven. Advanced technology should provide tools that facilitate and guide human information processing or decision making rather than dominate or replace human activities.

Collaborative human-machine problem solving should be incorporated into an automated information system environment for the IAEA. Expected benefits of further development and refinement of these ideas, and their eventual implementation, include

- improved operability and system effectiveness

- more consistent usage and outputs of the process

- decreased training requirements.

\section{Publication}

Greitzer, F. L., R. V. Badalamente, and T. S. Stewart. 1993. Collaborative Human-Machine Nuclear Non-Proliferation Analysis. PNL-8922, Pacific Northwest Laboratory, Richland, Washington. 


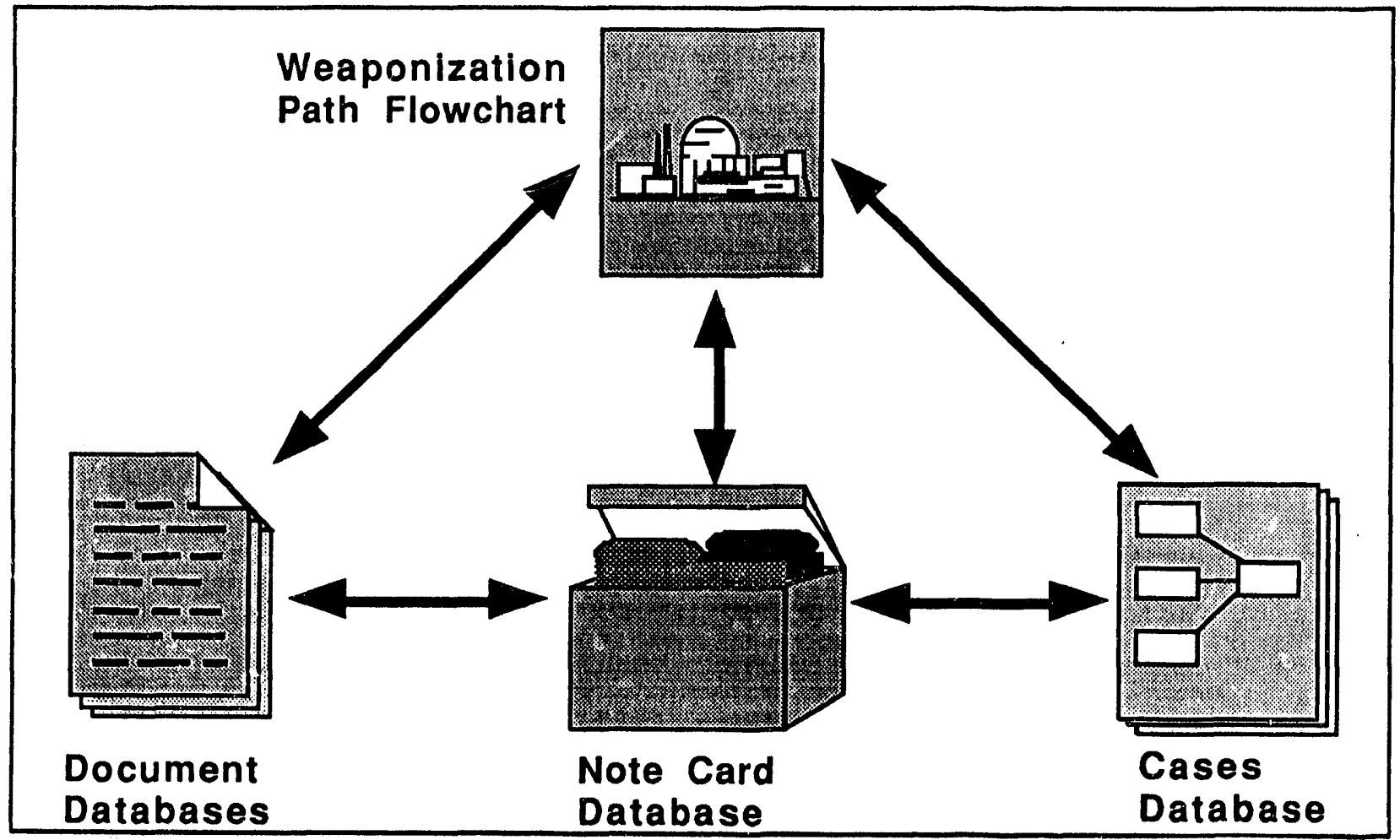

Figure 1. Links Among Collaborative Support Functions for Safeguards Information Treatment 


\title{
Prototype for Integrating Total Energy Cycle and Product Life Cycle Assessments
}

\author{
K. K. Humphreys and M. D. Adickes (Technology Systems Analysis)
}

Environmental professionals, policymakers, and industry are intensely interested in having a means to look holistically at the environmental consequences associated with the life cycle of energy systems from cradle to grave. One developing procedure which is used to examine energy systems is total energy cycle assessment. A second developing procedure for examining processes and products is product life-cycle assessment. The results generated by both procedures support environmentally sound technology development, waste minimization, energy conservation, and environmental policy analysis.

This project involved conceptualizing and building a prototype computer tool that will support the integration of total energy cycle and product lifecycle data and assessment approaches. A welldefined prototype that can be enhanced as part of future analytical efforts was produced.

Both the total energy cycle and product life-cycle assessments fall into the general category of life-cycle assessments. In a life-cycle assessment, an analyst is interested in evaluating energy and environmental consequences associated with a technological system. The analyst initiates a life-cycle assessment by conducting an inventory assessment which involves quantifying all material and energy inputs and resultant material and energy (including residual) outputs through all life-cycle unit processes. Next, the inventory is translated to impacts and subsequently, system improvements are made.

Existing computer tools that support life-cycle assessments are relatively inflexible in terms of data entry requirements (fixed units, mandatory sequential entry) and results presentation (fixed screen formats, limited output reports). Further, the user interfaces are difficult to use, calculations conducted by the tools are not always transparent, and data references and data quality are usually not addressed. These limitations are generally due to low quality user interfaces, overly restrictive data structures and restrictive modeling approaches that arose from a single-client focus during the original software development, and the limited software capability that was available at the time the models were built.

This effort took a step back from existing tools, and using a rapid prototyping approach, develop a concept for a significantly more flexible modeling system that would effectively handle product and energy systems with considerable flexibility on the data input side of the model, transparent calculations, a graphical user interface, and flexible user-specified results presentation and report generation. The inventory (not impact or improvement) components of a life-cycle assessment were the focus of the prototyping effort.

\section{Computer Prototype Advances}

The components of the prototype modeling system were defined to include a graphically-based scenario builder, stand-alone unit process data base, graphically-based report generator, and computational engine (see Figure 1).

The scenario builder is the primary portion of the modeling system that a user interacts with most frequently. It allows a user to select unit process profiles from the stand-alone data base and graphically arrange them into scenarios for further numeric and qualitative evaluation. Concepts for the unit process data base were developed to allow for flexible dats structures and data quality tracking to ensure fuli traceability of results. The report generator concept is to provide full analysis documentation in a variety of default and user specified formats. The model's engine does all the calculations and would not be a user-viewed part of a fully implemented modeling system. 
The functioning prototype was constructed using VisualBasic, which is an IBM, Windows-based programming environment. (Figure 2 presents several of the prototypes graphically-based user interface screens.) During demonstration of the prototype to
DOE and EPA staff, life-cycle assessments consultants, and private industry representatives, it has always been well received as a needed product to support environmentally-sound decision making in the public and private sector.

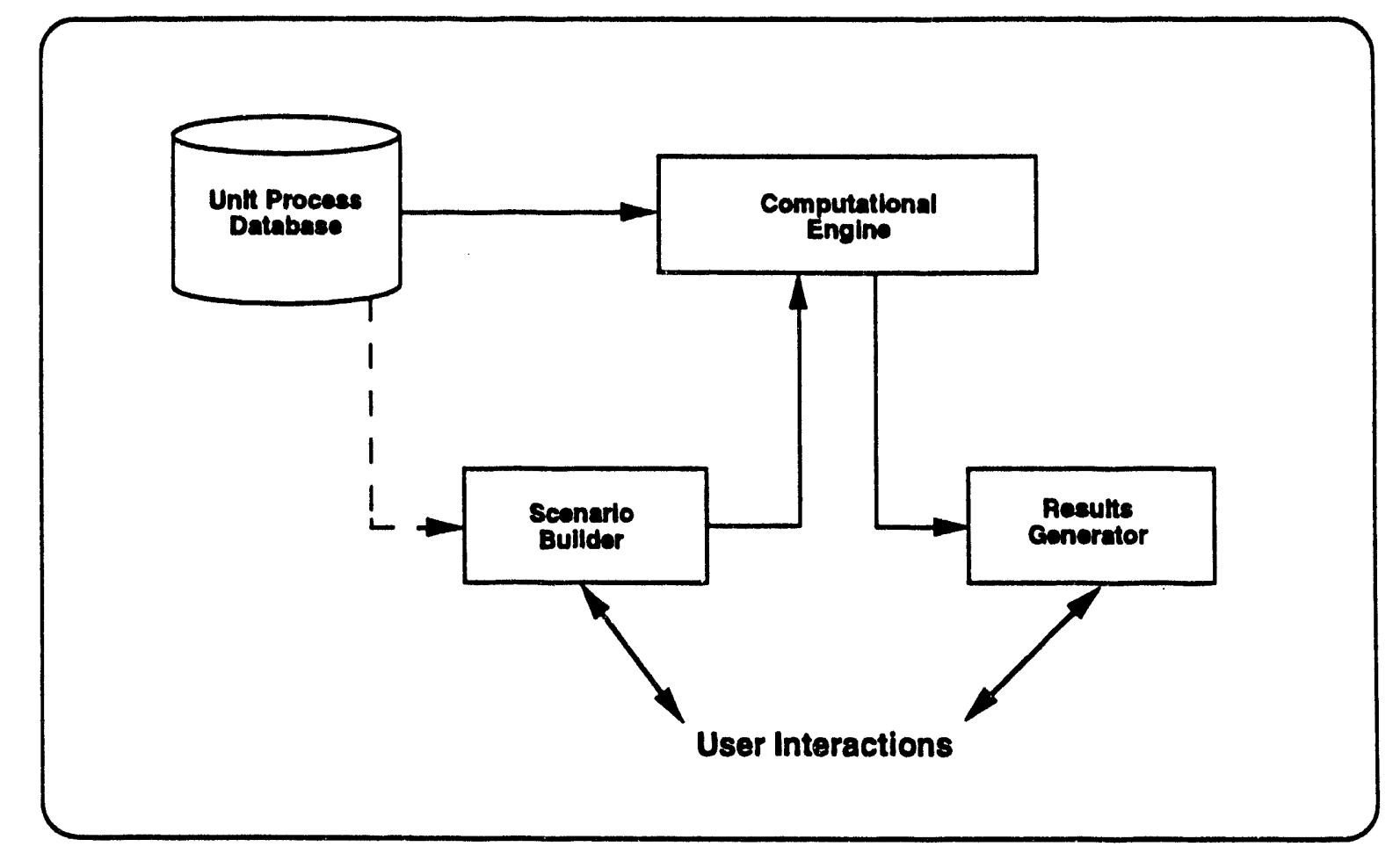

Figure 1. Four Major Components of a Lifo-Cycle Assessment Inventory Model 


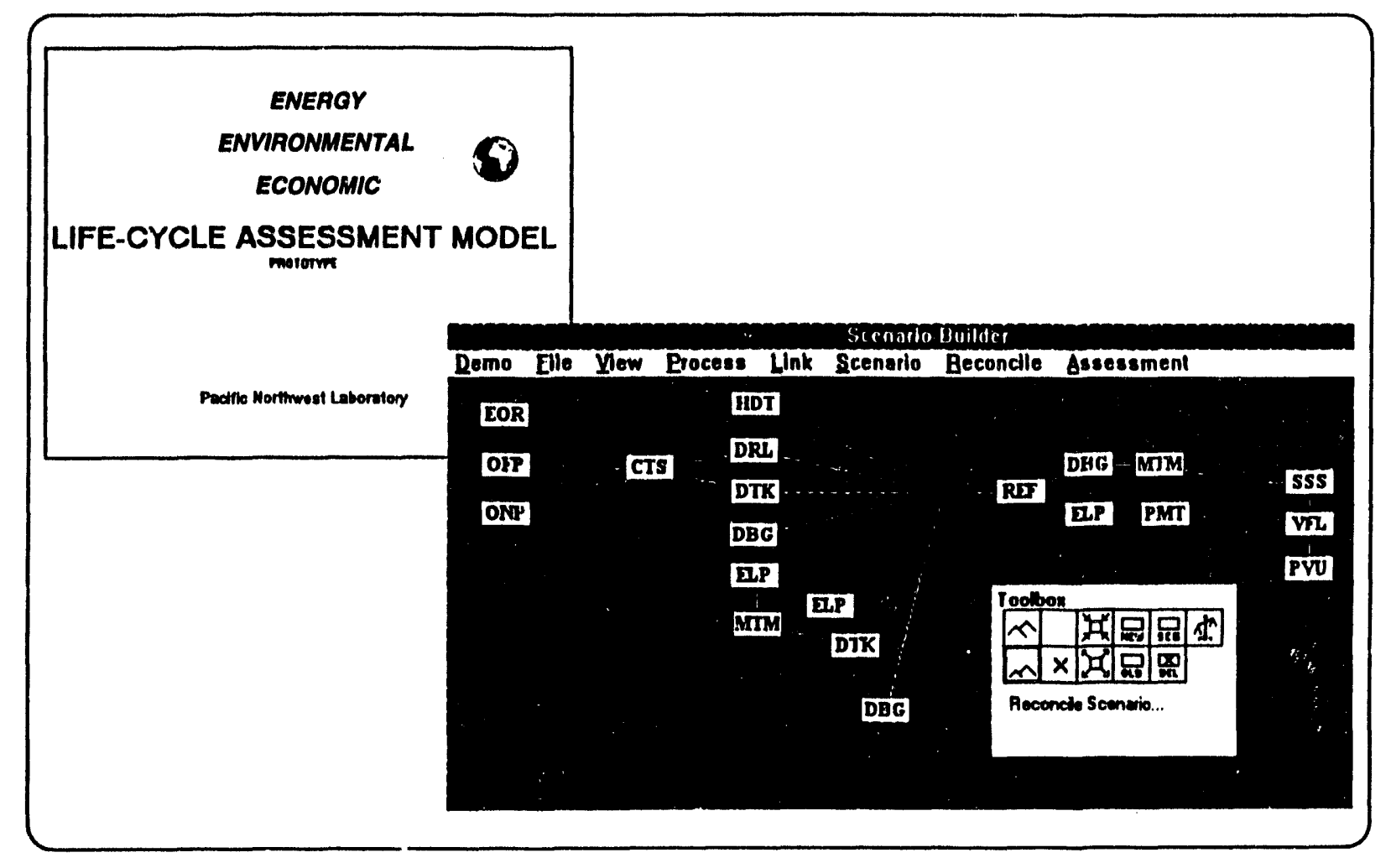

Figure 2. Sample Prototype Model Screens 


\subsection{Waste Technology}




\title{
Cavitation in Supercritical Carbon Dioxide
}

\author{
L. M. Bagaasen (Waste Process Engineering)
}

This project studied the effects of ultrasonic agitation in supercritical carbon dioxide $\left(\mathrm{SCCO}_{2}\right)$. Qualitative tests showed that ultrasonic agitation in supercritical carbon dioxide created peening patterns on aluminum foil similar to those caused by cavitation in aqueous systems. Quantitative tests showed that supercritical carbon dioxide with ultrasonic agitation removed as much as four times more contaminant than tests conducted without agitation.

Surface cleaning of metal, plastic, and composite precision parts is a critical operation in many manufacturing processes. These surface cleaning processes often rely on halons, chlorofluorocarbons (CFCs), carbon tetrachloride, and other fully halogenated alkanes. These solvents are being phased out because they are suspected stratospheric ozone depleting agents.

Supercritical carbon dioxide is a nonhazardous cleaning media that can be used to replace the ozone depleting solvents used in many of these cleaning processes. However, some contaminants, especially viscous, waxy, long-chained hydrocarbons are difficult to remove. Cavitation generated with ultrasonic agitation in conventional aqueous cleaning systems can significantly improve the effectiveness of aqueous cleaning processes. This work examined the possibility of using ultrasonic agitation to improve the cleaning capabilities of supercritical carbon dioxide.
The first set of tests attempted to determine if cavitation-like phenomena occur in supercritical carbon dioxide. In conventional aqueous cleaning processes, the intensity of peening patterns on aluminum foil is used to indicate the amount of cavitation occurring in the system. Aluminum foil was placed in the test apparatus with water, and peening patterns were obtained. Tests were repeated with supercritical carbon dioxide at 1500 psi and temperatures that ranged from $57^{\circ} \mathrm{C}$ to $76^{\circ} \mathrm{C}$. In all cases, peening patterns occurred on the foil that were similar to those obtained in the aqueous media. This positive result indicated that ultrasonic agitation might improve supercritical carbon dioxide cleaning processes.

The second set of tests investigated the removal of contaminants from stainless steel coupons. Lipstick is a common cleaning problem for precision parts and was chosen because it was known to pose a problem for supercritical carbon dioxide. Control tests showed that supercritical carbon dioxide could remove only $21 \%$ of the contaminant after a 10-minute cleaning cycle. Supercritical carbon dioxide with ultrasonic agitation removed $79 \%$ of the contaminant in a 10-minute cleaning cycle.

These two sets of initial results indicate that ultrasonic agitation could be used to improve the cleaning capabilities of supercritical carbon dioxide. 


\title{
CEPOD Treatment of Hazardous Organics
}

\author{
J. E. Surma (Waste Technology)
}

This work focused on the application of catalyzed electrochemical oxidation of hazardous organic compounds. The process has been previously demonstrated on nontoxic organics and been shown to be effective for the complete destruction to $\mathrm{CO}_{2}$ and $\mathrm{H}_{2} \mathrm{O}$.

A new approach to the destruction of chemical weapons was demonstrated at laboratory scale. This technique involves the low temperature electrochemical oxidation of hazardous organic compounds. The specific technology employed is known as the catalyzed electrochemical oxidation process (formerly Catalyzed Electrochemical Plutonium Oxidation and Dissolution [CEPOD]). This technology was developed at PNL and originally used to dissolve highly refractory oxides, but has since been applied to the treatment of hazardous organic compounds. Work has been completed using actual chemical surety material to evaluate the utility of this technology as an alternate method to destroy chemical warfare agents. The experimental work involving the actual nerve agents was performed at Battelle's Hazardous Materials Research Facility located in West Jefferson, Ohio. This technology can be used as an alternative to traditional incineration to oxidize the organic compounds completely to carbon dioxide and water.

\section{Application of Catalyzed Electrochemical Oxidation to Chemical Weapons Destruction}

For the destruction of hazardous organics, such as the chemical warfare agents, a complete electrochemical oxidation process with ancillary equipment is required. The process comprises an electrochemical cell, an ultrasonic generator, a pump, and a gas/liquid separator. Additionally, a simple off-gas treatment system is used to ensure all gaseous effluents are acceptable for discharge to the environment. All of the above-mentioned components are commercially available.
Presented below is the example of the electrochemical oxidation of sarin (GB):

$\mathrm{C}_{4} \mathrm{H}_{10} \mathrm{FO}_{2} \mathrm{P}+10 \mathrm{H}_{2} \mathrm{O} \rightarrow \mathrm{H}_{3} \mathrm{PO}_{4}+\mathrm{HF}+4 \mathrm{CO}_{2}+$

$26 \mathrm{H}^{+}+26 \mathrm{e}^{-}$

The balanced chemical equation shows that 26 Faradays of charge are required for pvery mole of GB oxidized to $\mathrm{CO}_{2}$ and the corresponding inorganic acids. This corresponds to an estimated power consumption of $10,000 \mathrm{kWh} /$ ton of agent. It should be noted that many organics can be processed in this electrochemical system at near $100 \%$ columbic efficiency, i.e., the amount of power actually used versus the theoretical power required to completely oxidize the organic to $\mathrm{CO}_{2}$ and $\mathrm{H}_{2} \mathrm{O}$, and possibly inorganic acids. The power consumption is relatively high; however, the system is very simple and, therefore, has a correspondingly low capital cost when compared to incinerators.

The catalyzed electrochemical oxidation process could be easily sized to fit the desired application. It is also amenable to modular and transportable systems due to the relatively simple design. The process is very benign in that typical operating conditions involve temperatures in the range of $30^{\circ} \mathrm{C}$ to $80^{\circ} \mathrm{C}$ and ambient pressures. Oxidation of the chemical warfare agent to carbon dioxide, water, and inorganic acids is accomplished in the same manner as the traditional incineration process, but at low temperature and low pressure.

Due to the relatively benign conditions at which the total oxidation of the chemical warfare agent takes place, this technology is an inherently safe process. Unlike incineration, during a process upset, the power to the electrochemical oxidation process can be interrupted and all reactions cease. Electrochemical oxidation is a very simple process and does not require a complex off-gas treatment system, as do high temperature processes like incineration. Therefore, the electrochemical oxidation process could replace such high temperature processes. 


\section{Results}

The results of these preliminary tests indicate that the catalyzed electrochemical oxidation technology is a promising low temperature alternative to the incineration of GB. It was shown through analysis of the offgas that under continuous processing of agent in the catalyzed electrochemical oxidation, destruction efficiencies of better than six 9s (99.9999\% destroyed) could be achieved. This work clearly indicates that further investigation and optimization of the catalyzed electrochemical oxidation technology would provide a useful alternative to incineration for the destruction of chemical warfare agents. 


\title{
Electrokinetic Distribution of Nutrients to Enhance In Situ Bioremediation
}

\author{
M. J. Truex (Waste Treatment Technology) and J. W. Virden (Materials Sciences)
}

This project investigated the delivery of nutrients in the subsurface using electrokinetic phenomena to control water and nutrient movement in the soil. Water movement was initiated by applying a dc-voltage between electrodes in soil. Due to the negative surface charge of the soil particles, water and positive ions will flow toward the cathode while negatively charged ions will migrate toward the anode.

As the first task in this project, an electrokinetics cell was designed and built. The design was based on test cells reported in the literature (Probstein and Shapiro 1990; Shapiro and Probstein 1993).

Experiments were performed using dehydrated pure kaolinite clay. Characteristics of the clay were determined to be approximately as described in Shapiro and Probstein (1993). For each experiment, the column was packed with clay rehydrated with a $0.1 \mathrm{M} \mathrm{NaCl}$ solution.

Calculations and equations are based on the results and parameters listed in Shapiro and Probstein (1993) because of the similarity to experimental methods used in this study. The following system parameters are used throughout this paper: tortuosity $=1.24$, permittivity $=10^{-9} \mathrm{C} / \mathrm{V} \cdot \mathrm{m}$, and viscosity $=10^{-3} \mathrm{~Pa} \cdot \mathrm{s}$.

\section{Experimental Results}

Results for nitrate experiments are listed in Table 1. Note that breakthrough occurs around 24 hours in both experiments. The calculated ratio of ion migration velocity to electroosmotic velocity is between 2.5 and 4.3. The magnitude of the ion migration velocity is 1.37 to 1.57 times higher than the generic migration velocity reported in Shapiro and Probstein (1993). Expected differences in the observed ion migration velocity as a result of variation in the experimental parameters (acetate versus nitrate ion, $\mathrm{pH}$ effects, clay material packing, experimental apparatus [primarily electrode materials], and experimental error) are well within this observed difference. From these results it appears that the equations developed in Shapiro and Probstein (1993) are valid for application to other experimental systems.

Four species were used as representative nutrients in Tabie 2 to illustrate the relative effects of species properties on the length of influence. For each species, a base scenario is listed as the first entry. In the following entries, the effect of varying electric potential, biomass concentration, zeta potential, and nutrient concentration are illustrated. The primary factor affecting the length of influence is the biomass concentration because it varies over several order of magnitude between background and moderate biofilm conditions. The electric potential also has a large effect for ionic species, but it is overshadowed by the effect of biomass concentration. Zeta potential has a relatively small effect because electroosmotic velocities are small compared to ion migration and extremely small relative to reaction rates. The only scenarios where nutrients can be moved greater than 10 meters is where the biomass concentration is at background levels. While the length of influence does increase with increasing electric potential, the system costs also increase.

Testing was problematic for several reasons. A primary problem with the cell design was the use of carbon steel electrodes. Using this material for the anode resulted in heavy corrosion. This hampered both analyses and potentially, electrokinetic effects in the clay due to corrosion products interfering with the surface charge of the clay and/or possible changes in the double layer. Two changes in electrode design have been proposed and implemented by other researchers at PNL as part of the In Situ Remediation Integrated Program electrokinetics project started in the summer of FY 1993. A tin oxide electrode i.ds been used without experiencing the corrosion difficulties. As well, a graphite electrode has been applied using a modification of the electrode design in the cell from this project. 
The second major problem was the application of the column in a horizontal orientation. Because of the inherent difficulties in packing rehydrated clay, fissures often formed due to settling of the clay over the colrse of the experiment. The initial reason for a horizontal orientation was to equalize the hydraulic head of the influent and effluent. However, upon closer inspection of the problem, it is evident that a vertical column could be employed without the problem of hydraulic head differences or gravity interference.

\section{Conclusions}

Electrokinetics was shown to have some limitations relative to moving nutrients into low permeability areas for use in bioremediation systems. The primary limitation is due to the low flux of nutrients compared to the utilization rate for many bioremediation systems. While in some instances, nutrients such as phosphorus and ammonia could be delivered using electrokinetics at a rate exceeding the assimilation of these compounds, the problem of delivering the remaining compounds necessary for successfully implementing bioremediation would not be solved. In the present state of development, electrokinetic fluxes of substrates and/or electron acceptors are not fast enough to maintain reasonable levels of metabolism for bioremediation applications once the biomass has increased above normal background levels. Electrokinetic distribution of bacteria was not studied as part of this project, but has potential for some applications of bioremediation.

However, application of electrokinetic distribution of nutrients in the vadose zone is promising. Electrokinetics can be used to alter water potential of the soil and can distribute necessary nutrients for assimilation while an alternate means, gaseous distribution, can be used to deliver the substrate and/or electron acceptor as necessary. In this scenario, electrokinetics would be used to prepare a site by increasing the water potential and supplying a source of assimilable nitrogen and phosphorus. After this site preparation, gaseous compounds would be distributed to start the bioremediation process by stimulating microbial tietabolic activity. With this strategy, the electrokinet:c distribution rates would not need to be as high as would be necessary to support an active microbial population during transport. Distribution would occur before significant activity begins but would support vigorous activity by providing good initial conditions for microbial metabolism and growth, and hence, contaminant biodegradation.

Key to applying this technology in the vadose zone is determining the relative flux of water induced by electroosmosis and balancing this with water addition to achieve the desired final water potential. Because water potential gradients would be established in the vertical direction due to the water introduced into the treatment zone in the unsaturated soil, vertical migration of water and potentially contaminant must be carefully assessed and controlled.

References

Probstein, R. F., and A. P. Shapiro. 1990. Electroosmotic Decontamination of Hazardous Waste Sites. Chemical Processing. Nov., p. 35-40.

Shapiro, A. P., and R. F. Probstein. 1993. "Removal of Contaminants Form Saturated Clay by Electroosmosis." Environ. Sci. Technol. 27:283-291. 
Table 1. Nitrate Ion Migration Test Results

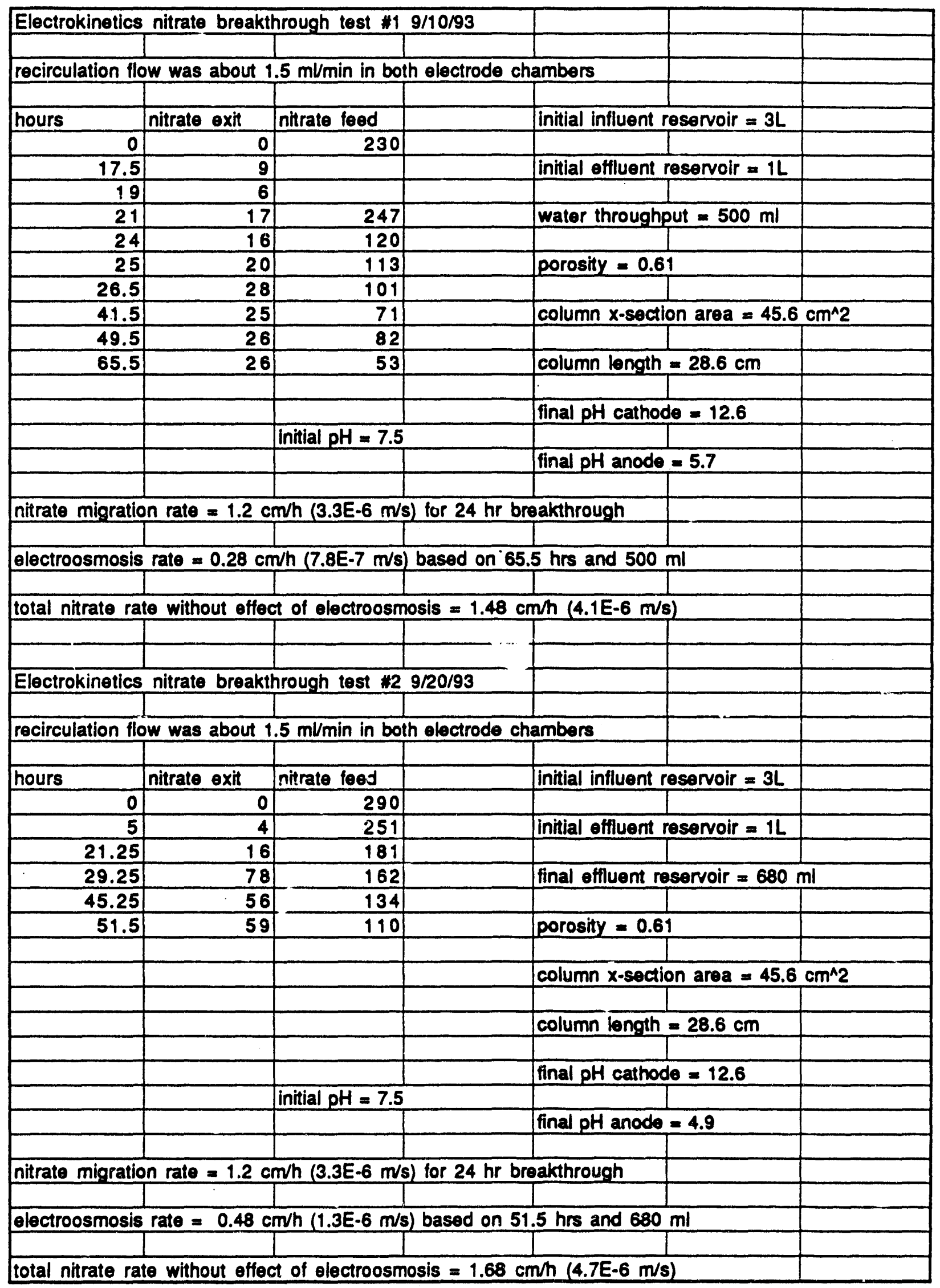




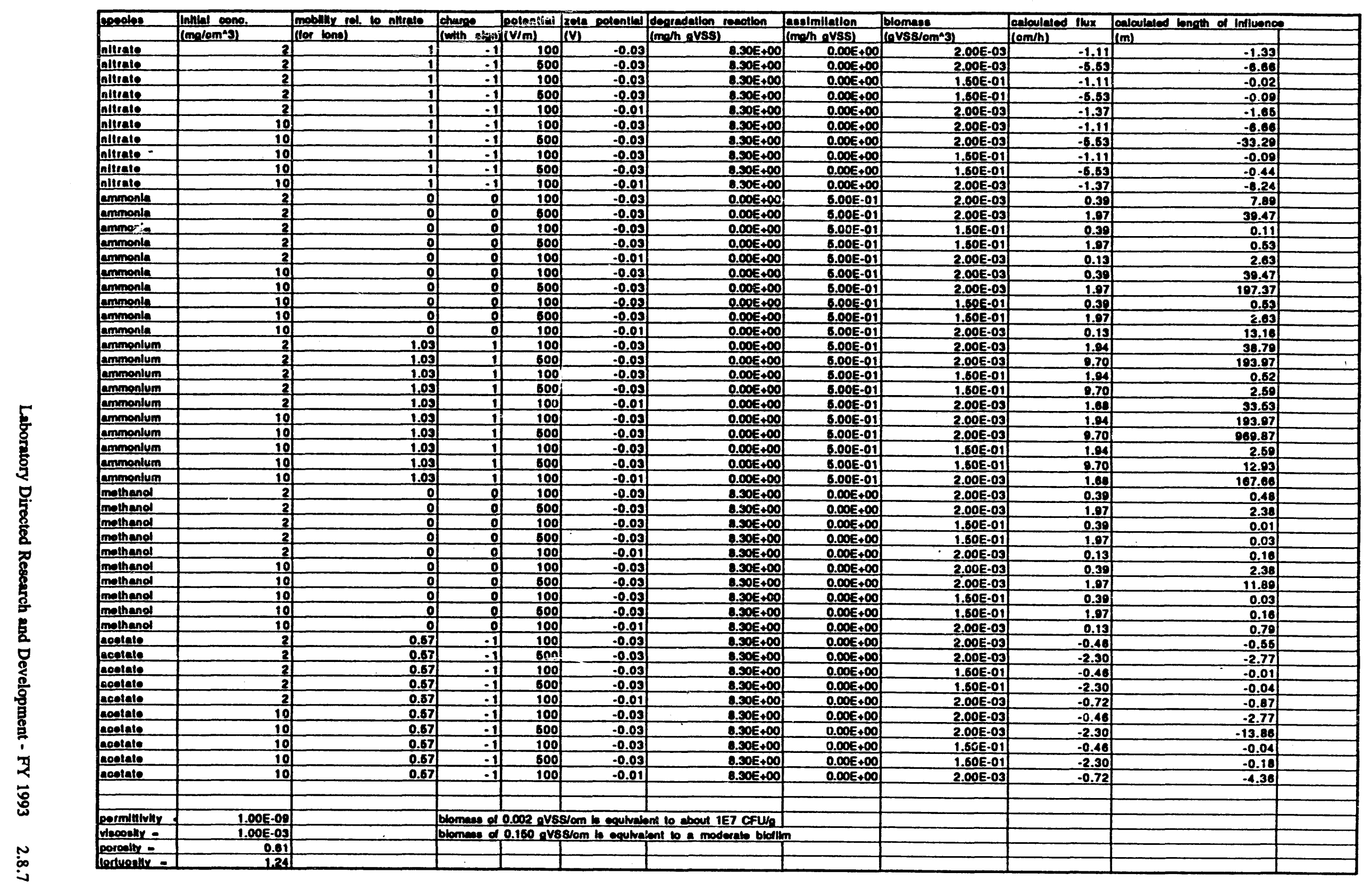




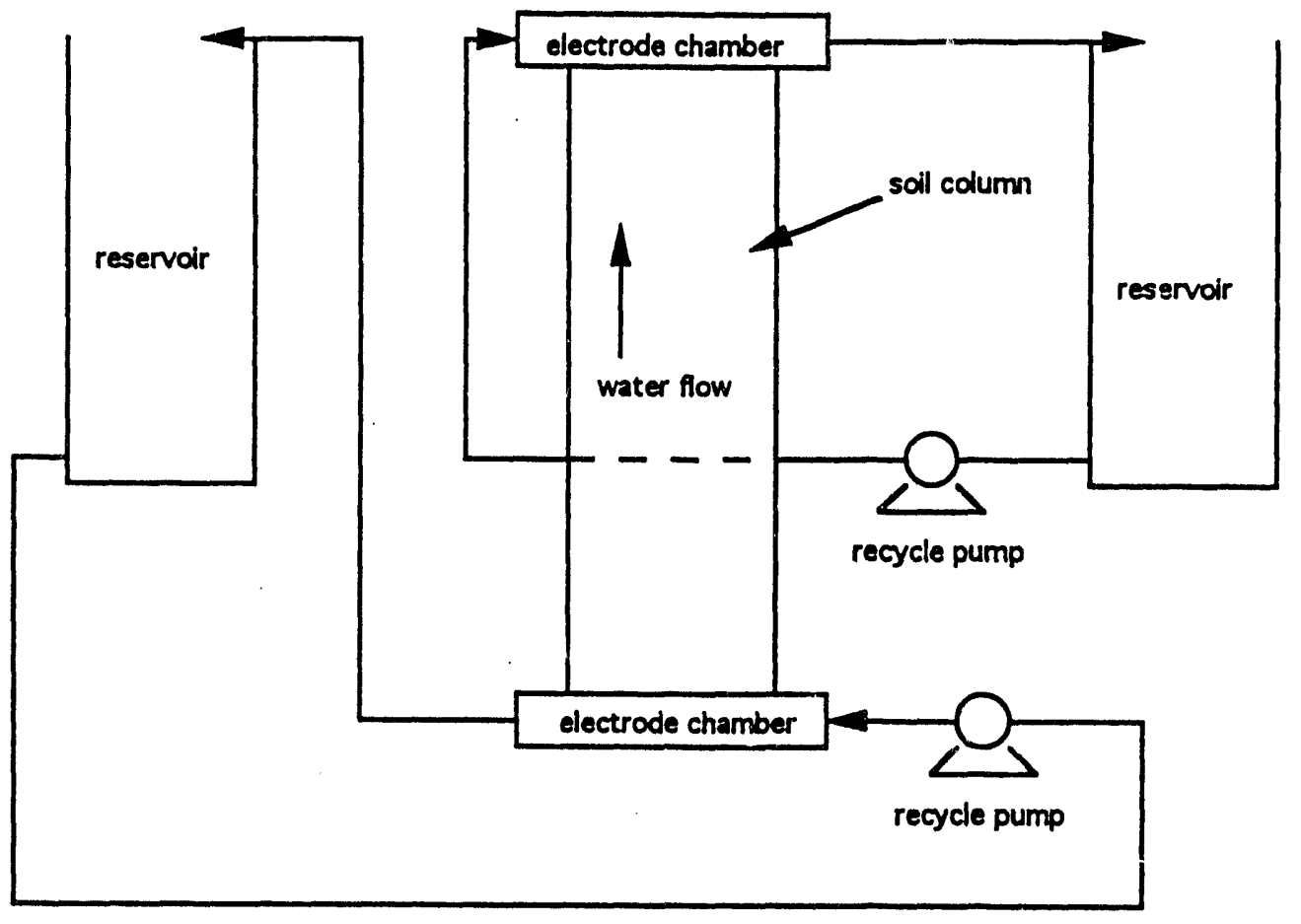

Note: Gravity is counteracted because the discharge height for both electrode chambers is equal.

Figure 1. Upflow Column Configuration for Electrokinetic Experiments

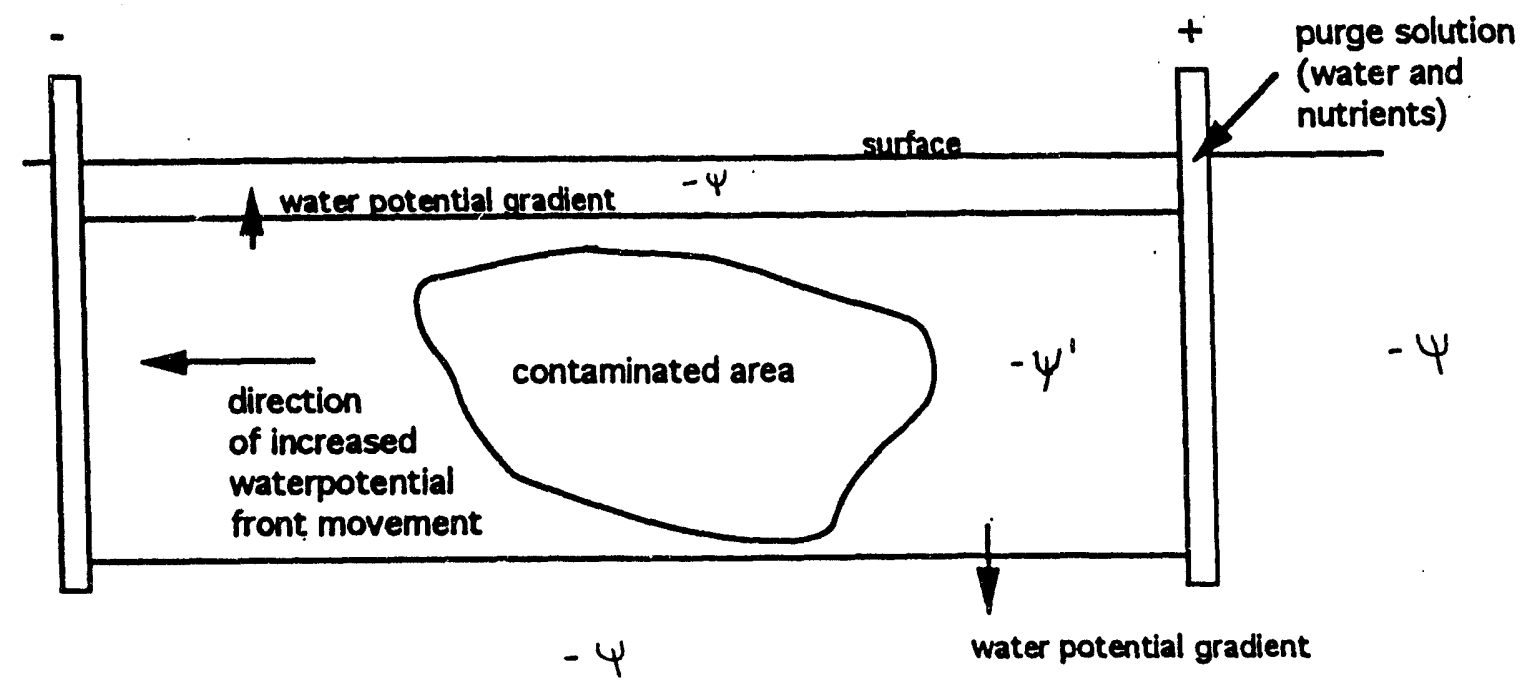

groundwater table

$$
-\psi^{\prime}>-\Psi
$$

Figure 2. Conceptual Application to Vadose Zone Bioremediation 


\title{
Engineering Transport Phenomena
}

\author{
G. Pillay, P. A. Gauglitz, and L. M. Peurrung (Waste Treatment Technology)
}

Several technologies being developed within the Engineering Technology Center rely on understanding and properly engineering the transport processes in porous media. These technologies include in situ bioremediation, bioventing, and six-phase soil heating. The objective of the first section of this work is to evaluate the detachment processes of biofilms within porous media. The second section of this project involves the development of a dual-gas tracer technique to characterize diffusion limitations in porous media during gas injection processes such as bioventing, soil vapor extraction, and six-phase soil heating. This work will give PNL state-of-the-art information on these important transport phenomena.

\section{Biomass Transport Processes in Porous Media}

The biotransformation of hazardous materials in both laboratory soil column reactors and in situ depends on the accumulation and activity of microorganisms. In porous media systems, most of the microbial activity is associated with microbes attached to soil surfaces. Effective field-scale in situ bioremediation requires a better understanding of the processes that affect the accumulation and activity of the attached biomass performing the desired contamizait transformations.

At the present time, a computer simulation is the only tool available that can incorporate data from the microbial, geological, and chemical characterizations to aid in the design of an in situ bioremediation system. Therefore, if the design of the remediation process is to be based on the results of computer simulations, the simulations themselves must be as accurate as possible, and the model must be used in a purely predictive manner. The primary goal of this LDRD project was to improve the accuracy of model predictions through an improved mathematical description of the processes that affect biomass accumulation in porous media.

The approach described uses data obtained from an aerobic differential packed bed reactor using glucose as the electron donor to determine kinetic parameters for attachment and detachment expressions, and then applies the derived model to predict results observed in a soil column using acetate as an electron-donor under denitrifying conditions. The experiments were performed in different laboratories for two independent experimental programs.

The numerical simulation was calibrated with data obtained from a packed bed biofilm reactor. Simulation results gave good correlation with the raw data for both liquid phase substrate and biomass concentration. The simulation results for total column biomass did not fit as closely as desired. At the present time expe rimental results from PNL denitrification experimenis have not yet been simulated. Because of the complexity of the model, its development and calibration has required more time than expected.

The development of the kinetic model, which incorporates the effects of cellular attachment, detachment, and filtration while at the same time calculating a pore size distribution to predict well fouling and formation plugging has improved the Engineering Technology Center's capability for prediction of subsurface biomass accumulation and activity. This will improve our understanding of subsurface transport, in situ bioremediation, and our ability to obtain outside funding for further research in this area, while improving our collaborative ties with the National Science Foundation Engineering Research Center for Biofilm Engineering (CBE) at Montana State University.

Our success in simulating the $2 \mathrm{mg} / \mathrm{L}$ glucose results from CBE gives credibility to the initial formulation of the equations and assumptions used to develop this model. Further research is needed to verify the accuracy of the simulation.

\section{Dual-Gas Tracer Technique}

Funding was approved for experimental equipment to develop the dual-gas tracer technique for quantifying diffusion limitations in soil remediation. The initial focus of the dual-gas tracer technique will be sixphase soil heating, a technology undergoing aggressive development añ marketing by the Advanced 
Technologies Group. The funded experimental equipment included a large oven, pumps, mass flow controllers, gas chromatograph, computer, and associated plumbing. During FY 1993, construction of this apparatus was begun, along with training for the technical investigators regarding the operation of the process control computer and its associated programs.

An invention disclosure was also filed during FY 1993. Our invention was the use of the multiple gas tracer technique originated by Radke and Gillis to study mass transfer between stagnant liquid held in saturated, clay-like materials and streams of mobile gas in the surrounding high-permeability regions. We have chosen fluorinated gases $\left(\mathrm{C}_{2} \mathrm{~F}_{6}, \mathrm{SF}_{6}, \mathrm{C}_{2} \mathrm{~F}_{4}\right.$, and $\mathrm{CHF}_{3}$ ) with a wide range of water solubilities to inject as tracers. These tracers are detectable at very low concentrations (as low as parts per billion) using a gas chromatograph with an electron capture detector. Another planned application is the use of gas tracers to study gas diffusion limitations in soils. Gas tracers were chosen to be identical except for their gas-phase diffusivities, and examples of these tracers include $\mathrm{CF}_{4}, \mathrm{C}_{2} \mathrm{~F}_{6}$, and $\mathrm{C}_{3} \mathrm{H}_{8}$.

Packing materials for the soil columns were also chosen and obtained. Four sieve fractions of an Ottawa sand will be used for the high-permeability, low liquid-saturation fraction. The low-permeability, high liquid-saturation materials will be Berea sandstone cores or a porous stainless steel material in the form of a tube or thin sheets.
We anticipate that the bench-scale tests of the dual-gas tracer technique will ultimately result in its use as a field-scale site characterization method. Data interpretation for the bench-scale and especially the fieldscale work will require mathematical modeling of mass transfer processes in unsaturated soil. This work was also begun in FY 1993. We chose the Multiple Subsurface Transport Simulator (MSTS) developed by PNL staff for the Yucca Mountain Project for its ability to simulate the movement of water-soluble gases through heterogenec is, unsaturated porous media. The code has been implemented on a workstation in the Advanced Technologies Group, and its numerical solutions of one-dimensional problems have been checked versus analytical solutions for correctness. The code requires a large number of physical properties (diffusivities, permeabilities, porosities, dispersivities, etc.) for each soil and tracer gas. An extensive literature search was performed to find values and correlations for our materials.

Funding for FY 1994 will allow us to continue the installation of the apparatus and to begin experimental work on a variety of soils using the suite of gas tracers that were identified in the previous fiscal year. The modeling eifort will also continue and will incorporate the experimental data taken from the apparatus. 


\title{
Enhanced Bioremediation Using In Situ Heating
}

\author{
W. O. Heath (Waste Technology)
}

The objective of this project was to complete tests to quantify the ability to accelerate in situ bioremediation of organics through the use of lowenergy, in situ soil heating. Bench-scale experiments were conducted to evaluate the rate of biodegradation of a simple organic compound under anaerobic conditions, with and without electrical heating, during the first phase of this project.

Labortory experiments were performed to attempt to quantify the degree to which microbial activity can be enhanced by electrical heating below the water table. The concept is to increase and maintain in situ temperatures at an optimum range of $30^{\circ} \mathrm{C}$ to $35^{\circ} \mathrm{C}$ to promote maximum metabolic growth rates for faster site cleanup. Uniform temperature profiles are critical, however, because the metabolic activity of bacteria may significantly decrease at tempe.atures above $50^{\circ} \mathrm{C}$. By heating the groundwater or vadose zone with sufficient uniformity, it would be expected that treatment times could be reduced by a factor of 4 or 5, based on the standard tempes.ture dependence of microbially-catalyzed reactions typically described using Arrhenium equations.

Experiments were conducted with a bench-scale soil reactor fitted with six electrodes connected to a sixphase ac power system. Radial temperature profiles established by six-phase heating ranged from $31.6^{\circ} \mathrm{C}$ to $34.9^{\circ} \mathrm{C}$ within the region inside of the electrode array, which were within the target range, with a typical variation of $\pm 1^{\circ} \mathrm{C}$ within this region at any given time.

The experiments indicated an increase in denitrification rate of about 5.7 to 6.1 from tests performed without heating at $10^{\circ} \mathrm{C}$. It was also confirmeci that biodegradation rates followed the Arrhenius equation for reaction rate temperature dependence, enabling direct prediction of increased contaminant removal.

These results suggest that six.-phase heating could be used to extend the applicability of bioremediation to cold environments where the ambient temperature is below $5^{\circ} \mathrm{C}$ to $10^{\circ} \mathrm{C}$, a temperature range where microbial activity in soil becomes extremely slow, and bioremediation had previously been ruled out. 


\title{
Low Temperature Plasma Applications
}

\author{
T. M. Bergsman and G. Pillay (Waste Treatment Technology)
}

The objective of this project was to determine the feasibility of using existing low-temperature plasma technology to oxygenate fuels and convert coal shale to low Btu (British thermal unit) fuel gas. This project reviewed the current state of the art and performed a chemical thermodynamics study to determine the feasibility of the proposed chemistry.

The 1990 Clean Air Act presents new emissions standards for mobile and stationary sources of air pollutants (Summers et al. 1992). The regulations in this Act necessitate the development of advanced vehicle fuels, such as oxygenated fuels for automobiles, to aid in the reduction of hydrocarbon emissions. In this project, a review of relevant literature was conducted to determine the feasibility of using existing low-temperature plasma technology at PNL to synthesize these fuels and to perform gasification of coal shale to fuel gas.

\section{Literature Review}

Conventional uses for low-temperature plasma discharges have been summarized previously (Goldman et al. 19.85) and include ozone synthesis, surface treatment of polymers, and various electrostatic applications (Clements et al. 1989), including the use of corona discharge for destruction of volatile organic contaminants (VOCs) (Ramsey et al. 1990). PNL researchers have also built and tested a unique corona discharge reactor to treat volatile organic contaminants (Virden et al. 1992). Because the corona discharge generates an extremely oxidative environment, it may be possible to use this low-temperature plasma for other applications in chemical synthesis, including the production of advanced fuels.

Fu et al. (1971) stury the use of microwave discharge plasma to gasify solio fuels, including bituminous coal and oil shale, to produce pipeline gas. They use a cylindrical Vycor tube as a flow reactor, in which the gas stream was exposed to the plasma discharge for 30 minutes. Product analysis was performed using a mass spectrometer. The authors observed that hydrogen and gaseous hydrocarbons, such as acetylene, ethylene, and higher molecular-weight hydrocarbons ( $\mathrm{C}_{8}$ and higher) were produced in appreciable amounts. Hydrocarbon formation was aided by using a hydrogen-argon discharge environment. This work shows that the use of plasma discharge to generate gaseous hydrocarbons from solid fuels is feasible. Therefore, it is possible that solid fuels exposed to a low-temperature plasma generated by corona discharge may also produce gaseous hydrocarbons.

Previous work by Ramsey et al. (1990), Virden et al. (1992), and Kipp et al. (1973) shows that corona discharge may be used to produce oxygenated species and activated iurms of oxygen that could be used in the synthesis of advanced fuels. These studies examined the use of corona discharge to convert hydrocarbons in air streams to less toxic products. Certain compounds, such as alcohols, are produced and are potentially applicable to oxygenated fuel synthesis. However, more highly oxygenated species, such as carboxylic acids or epoxides, may be undesirable. This work suggests that it may be feasible to use a corona reactor to oxygenate fuels by exposing these compounds to low-temperature plasma in a controlled atmosphere.

\section{Future Work}

Proof-of-principle experiments in the existing PNL corona discharge reactor are required to determine process feasibility. Modifications of this apparatus may be required to conduct these experiments. Corona byproducts should be analyzed to determine the overall success of the oxygenation reactions occurring in the corona device. Modeling and simulation work will also enhance the understanding of the corona discharge process chemistry. This work will allow researchers to examine the effects of the dominant reactions occurring in the gas plasma, applied electric field strength, and electron density in order to determine the impact of these process variables on current and future reactor configurations (Bell 1971; Mukkavilli et al. 1988). 


\section{References}

Bell, A. T. 1971. "Models for High Frequency Electric Discharge Reactors." In Engineering, Chemistry, and Use of Plasma Reactors, ed. J. E. Flinn, Chemical Engineering Progress Symposium Series, Vol. 67, No. 112, p. 1, American Institute of Chemical Engineers, New York.

Clements, J. S., A. Mizuno, W. C. Finney, and R. H. Davis. 1989. "Combined Removal of $\mathrm{SO}_{2}, \mathrm{NO}_{x}$, and $\mathrm{Fly}$ Ash from Simulated Flue Gas Using Pulsed Streamer Corona." IEEE Trans. Indus. Applns. 25(1):62.

Fu, Y. C., B. D. Blaustein, and I. Wender. 1971 . "Gasification of Solid Fossil Puels in a Microwave Discharge." In Engineering, Chemistry, and Use of Plasma Reactors, ed. J. E. Flinn, Chemical Engineering Progress Symposium Series, Vol. 67, No. 112, p. 47, American Institute of Chemical Engineers, New York.

Goldman, M., A. Goldman, and R. S. Sigmond. 1985. "The Corona Discharge, its Properties and Specific Uses." Pure and Appl. Chem. 57(9):1353.

Kipp, E. B., K. A. Shelstead, and G. S. P. Castle. 1973. "An Electrochemical Flow Reactor for Oxidation of Hydrocarbon Pollutants" Can. J. Chem. Engng. 51:494.
Mukkavilli, S., C. K. Lee, K. Varghese, and L. L. Tavlarides. 1988. "Modeling of the Electrostatic Corona Discharge Reactor." IEEE Trans. Plasma Sci. 16(6):652.

Ramsey, G. H., N. Plaks, C. A. Vogel, W. H. Ponder, and L. E. Hamel. 1990. "The Destruction of Volatile Organic Compounds by an Innovative Corona Technology." In Proceedings: Eighth Particulate Control Symposium, Volume 1: Electrostatic Precipitators, p. 9-2, Electric Power Research Institute, Palo Alto, California.

Summers, J. C., J. E. Sawyer, and A. C. Frost. 1992. "The 1990 Clean Air Act and Catalytic Emission Control Technology for Stationary Sources." In Catalytic Control of Air Pollution: Mobile and Stationary Sources, eds. R. G. Silver, J. E. Sawyer, and J. C. Summers, ACS Symposium Series 495, p. 98, American Chemical Society, Washington, DC.

Virden, J. W., W. O. Heath, S. C. Goheen, M. C. Miller, G. M. Mong, and R. L. Richardson. 1992. "High-Energy Corona for Destruction of Volatile Organic Contaminants in Process Off-Gases." Presented at SPECTRUM '92, Boise, Idaho. 


\title{
Microtechnology Applications in Waste and Chemical Processing
}

\author{
R. A. Brouns (Waste Treatment Technology) and R. S. Wegeng (Waste Systems)
}

Information and experience in the microtechnologies area has identified opportunities relative to applying related techniques in the field of waste and chemical processing. In this project, initial investigations of micro-separations techniques were conducted.

One of the many applications where microscale technology shows promise is in the field of process engineering. Significant information and experience has already been achieved in the microtechnologies area at PNL and this has opened up some very nearterm opportunities for applying related techniques in the field of waste and chemical processing. During the past decade there have been significant advancements in the fabrication of micro-mechanical structures by the Karlsruhe Nuclear Research center (KfK) of Germany using their LIGA process (Lithographie, Galvanoformung, Abformung - a particular microfabrication technique the Germans developed). A distinguishing feature of this advancement has been the ability to conduct high aspect ratio etching. Such unique capability has also been demonstrated at PNL under the current studies on microtechnology for energy conversation.

The objective of this study during FY 1993 was to initiate studies in the area of microtechnology-based waste processing. Contact was made with the German researchers to gain additional information on the design, fabrication, and performance of their micronozzles, and to provide an avenue for possible future collaborations. Several reports and photographs of their equipment were obtained along with a better understanding of their overall research program.

A strategy for future research was outlined and decisions were made on how to focus initial investigations in the micro-separations area. Specific applications in several areas related to both waste management and energy efficiency were identified. Analysis of the German micro-nozzle concepts for separation of certain groundwater contaminants was initiated. 


\title{
Novel Single-Well Electrode for Electrokinetics
}

\author{
P. A. Gauglitz (Waste Treatment Technology)
}

Electrokinetics is a promising in situ technology that can transport water, ions, and organics through soil over macroscopic distances by the application of an electric field. This project investigated a novel single-well electrode, which may improve the ability to control the radial and vertical migration of the injected water and/or chemicals.

The mechanisms relevant to contaminant transport in soil are electroosmosis and electromigration. Electroosmosis is the flow of pore water while electromigration is the movement of ionic species. Application of electrokinetics enables removal of radionuclides from very low permeability soils that are typically untreatable by conventional pump injection and withdrawal techniques. In addition, electrokinetics can provide more uniform volumetric delivery of chemicals to these soils. To facilitate the removal of contaminants strongly bound to the soil, additives are injected at the anode electrode and flushed through the soil to the cathode electrode. A technique to uniformly deliver these additives, which are typically water or other nontoxic organics, to the soil must be devised and understood to allow successful and widespread application of technology.

A single-well electrode allowing fluid injection and withdrawal at different depths for a single well location required a new design. The conventional electrode configuration for electrokinetic soil treatment consists of two electrodes, an anode and a cathode, placed at different locations. The prototype single-well electrodes were designed and then machined from solid graphite. A special pressure transducer was also obtained to accurately measure soil permeabilities, because permeability is a critical factor influencing the success of a single-well electrode.

Tests to observe how a single well electrode distributes water soluble additives to the soil were delayed because the flow visualization equipment was used by another project. 


\title{
Technological Opportunities From Unproductive Waste Streams
}

\author{
A. Khawaja and D. Stapp (Waste Systems)
}

The objective of this project was to examine landfill waste streams that currently do not have value as recyclables or feedstocks for incinerators, identify technological and related barriers to their utilization for energy or feedstock materials, evaluate technological approaches to overcoming these barriers, and select five to ten approaches as candidates for further proof-of-principle research.

Analysis of the plastics waste stream was conducted and documented in a report entitled, Diverting Plastics From Landfills: Opportunities in the Making. This report focused on the production, use, and disposal of plastics in the municipal waste system. The report discussed options for generation, use, and disposal of plastics within the context of current technology and discussed the need for further technology developments. A briefing was provided to PNL staff and the report made available to interested parties.

Site visits to waste facilities in western Washington were conducted in March. Sites visited included Waste Management Inc. Recycling America facility in Seattle; Rebanco's materials recovery facility in
Seattle; the Recomp fully integrated facility in Whatcom County; the plastics research center at Western Washington University; the Skagit Country incinerator/landfill facility; and the Clean Washington Center in Seattle, and the Longview Fibre Company. Activities at the facilities were observed and discussions were held with staff concerning technological opportunities for enhancing waste management.

The concept for a regional conference focused on solid waste emerged from onsite visits. Contacts were made throughout the industry. Eighty-five individuals interested in solid waste management, mostly from the Pacific Northwest, attended the conference. Topics focused on future technology requirements in the area of landfill mining, bioremediation, composting, waste minimization, and plastics manufacture and recycling. The conference also focused (in part) on how industry approaches the need for new technologies and the requirements placed on performance. The conference clearly showed that industry, state and local governments, and universities are interested in partnering to effect technological solutions to solid waste problems.

Follow-on activities in FY 1994 will look to capitalize on these partnering opportunities. 


\title{
Vitrification Process Rate Enhancement Using DC Plasma Arc
}

\author{
R. D. Peters and J. E. Surma (Waste Technology)
}

The objective of this project was to determine the performance of heating in the melter near or in the cold cap as a means to boost feed rate. The scope of work involved designing and installing electrodes which fed through the melter lid. Localized heat was generatrd near the glass surface to increase the melting rate of incoming material.

\section{Equipment Design and Installation}

The pilot-scale ceramic melter was selected for testing purposes because it was available and had access ports on the melter lid for electrodes. An assembly was designed and fabricated for mounting two electrodes of 2-inch outer diameter. The assembly was mounted on a flange on the melter lid and the electrodes were installed vertically near the entry point for melter feed. Alternative electrodes of 2 -inch outer diameter inconel 690 rods were also used.

\section{Electrode Testing}

The two mounted graphite electrodes were connected to a $15 \mathrm{~kW}$ dc power supply (for arc welding) to test the passage of current and generation of heat in the idling pilot-scale ceramic melter. However, we were not able to generate an arc between the electrodes that was sustainable. Arc gap positioning is an important factor which was difficult to control with the electrode mount. Also, the voltage limit on the power supply was about $30 \mathrm{~V}$ dc which was considered inadequate to strike off an arc.

Lacking a sufficient dc power supply, it was then decided to use an available ac power supply. The graphite electrodes were attempted but current passage was weak owing to surface resistance at the melt/ graphite interface. Oxidation of the graphite was also beginning to show near the hot melt surface $\left(1100^{\circ} \mathrm{C}\right)$ in the atmospheric melter plenum. The inconel electrodes were then installed and it was possible to pass current and generate localized heat with the ac power supply.

\section{Melter Preparation and Testing}

The testing strategy initially called for slurry feeding the pilot-scale ceramic melter from left-over feed in tank 60. This required check-out of the melter power controllers and the off-gas system. This exercise revealed that control and instrumentation in the pilotscale ceramic melter were not operationally functional. The off-gas control valve which regulates melter negative pressure did not perform and recorders for melter power were not operating properly. The pilot-scale ceramic melter control and instrumentation is so antiquated that diagnosis and repair of it is considered too costly.

Testing was limited to applying power to the topentering electrodes which were spaced at about 5 inches at the melt surface. When about 10 to $15 \mathrm{~kW}$ were applied (estimated by the settings on the $37.5 \mathrm{~kW}$ power supply), the glass in the region between the electrode became white hot indicating about $1300^{\circ} \mathrm{C}$ to $1400^{\circ} \mathrm{C}$.

\section{Conclusion}

This limited study demonstrates that auxiliary heating electrodes can be used to raise the temperature at the melt surface region of a joule-heated melter. Localized temperatures can be accomplished to enhance the melting rate of feed material. However, the bulk glass temperature can remain much lower to accommodate temperature limitations of primary melter electrodes and refractory materials. Such techniques may be useful for high-capacity melters needed to vitrify Hanford low-level waste. 


\title{
X-Ray Film Optical Reader
}

\author{
P. N. McDuffie (Waste Systems)
}

In the U.S. this year, more than five women will die from breast cancer every hour of every day and over 20 will be diagnosed as having it. Early detection and treatment can reduce the mortality rate significantly. The primary breast cancer screening methods recommended by the National Cancer Institute are clinical breast examinations by a physician combined with $x$-ray mammography. The objective of this project was the demonstration of a concept for optically enlarging $x$-ray images, primarily for use by Radiologists (MDs) in assessing mammograms for the presence of disturbed structures associa.ed with breast cancer.

Mammograms are the only kind of $\mathrm{X}$-rays that require a radiologist to look at them with a magnifying glass, because they are trying to assess image sizes of $1 \mathrm{~mm}$ or smaller (microcalcification). Radiologists now have 20 to $25 \%$ "true positive" rate of analysis, which means that for every four women undergoing the emotional trauma of being biopsied, only one has cancer.

The current assessment technique involves using a light board to back-light the $x$-ray and a hand held $(2 X-4 X)$ magnifying glass for observing these small structural disturbances (spots about the size of this [.]). Recognizing that all the information available to the radiologist is contained in the $x$-ray film, optical enhancement appeared to offer the most acceptable method to present the images without degradation.

The design of a system using optical projection techniques to confirm a radiologist's assessment prior to biopsy was to be developed. However, the effort did not receive funding until early August. Due to lack of time and staff resources, only a proof-ofconcept demonstration was attempted.

\section{Technical Progress}

A $1 \mathrm{~mm}$ "spot" (between the "V," Figure 1a) representative of a microcalcification formation in a mammogram image was enlarged approximately $20 \mathrm{X}$ (Figure 1b) to show that the surrounding shades-ofgray, the texture, and edge conditions of the object can be presented for analysis by a radiologist without loss of information from the optical process. The upper limits of enlargement appear to be around $100 \mathrm{X}$ (Figure 2), where film grain starts to interfere with image quality. Unfortunately, the photos made using this prototype system and the loss of resolution of the printing process do not adequately portray the quality of the projected images.

A digital data base of the enlarged images, classified in accordance with the American College of Radiology's LEXICON and annotated with pathology results, would appear to be highly beneficial to both practicing physicians and for training purpo ss, and could be transmitted electronically to the American College of Radiology's National Mammography Database.

In its simplest form, a desk top unit for viewing only (no image storage capability), would cost less than $\$ 1,000$ per unit. The next step in the development involves a design with a variable intensity light source, improved $x$-y film positioning platform, zoom optics (10X-25X), and a digital recording capability (video or direct digital camera). Utilization of the resulting data appears almost limitless-cataloging images for LEXICON, training, screening mammography, etc. 


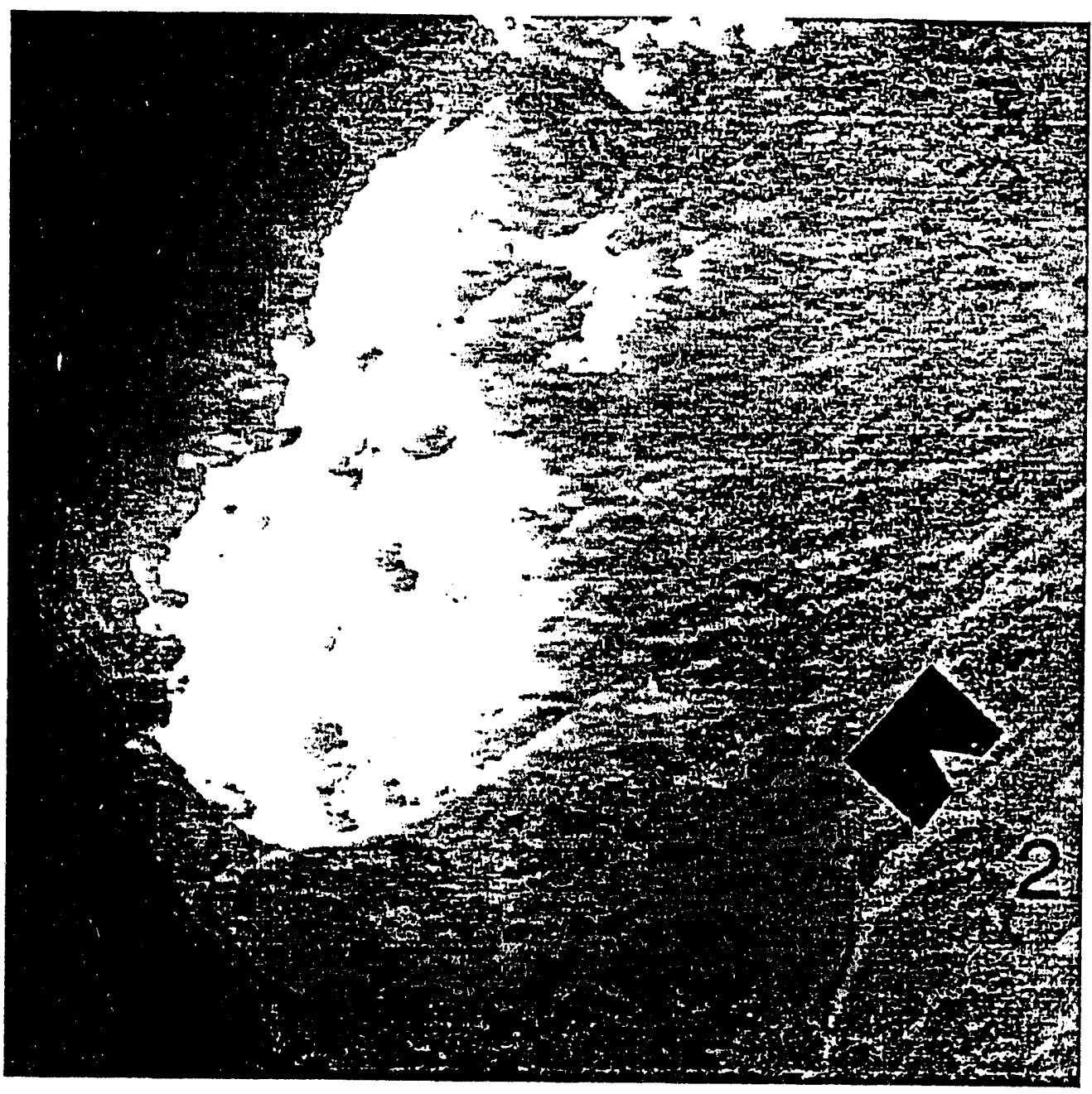

Figure 1a. Mammogram (2.5X) Showing Location of $1 \mathrm{~mm}$ "Spot" (between "V")

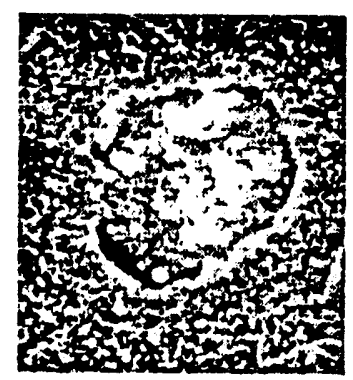

Figure 1b. $1 \mathrm{~mm}$ "Spot" (22X). Note texture and edge detail. 


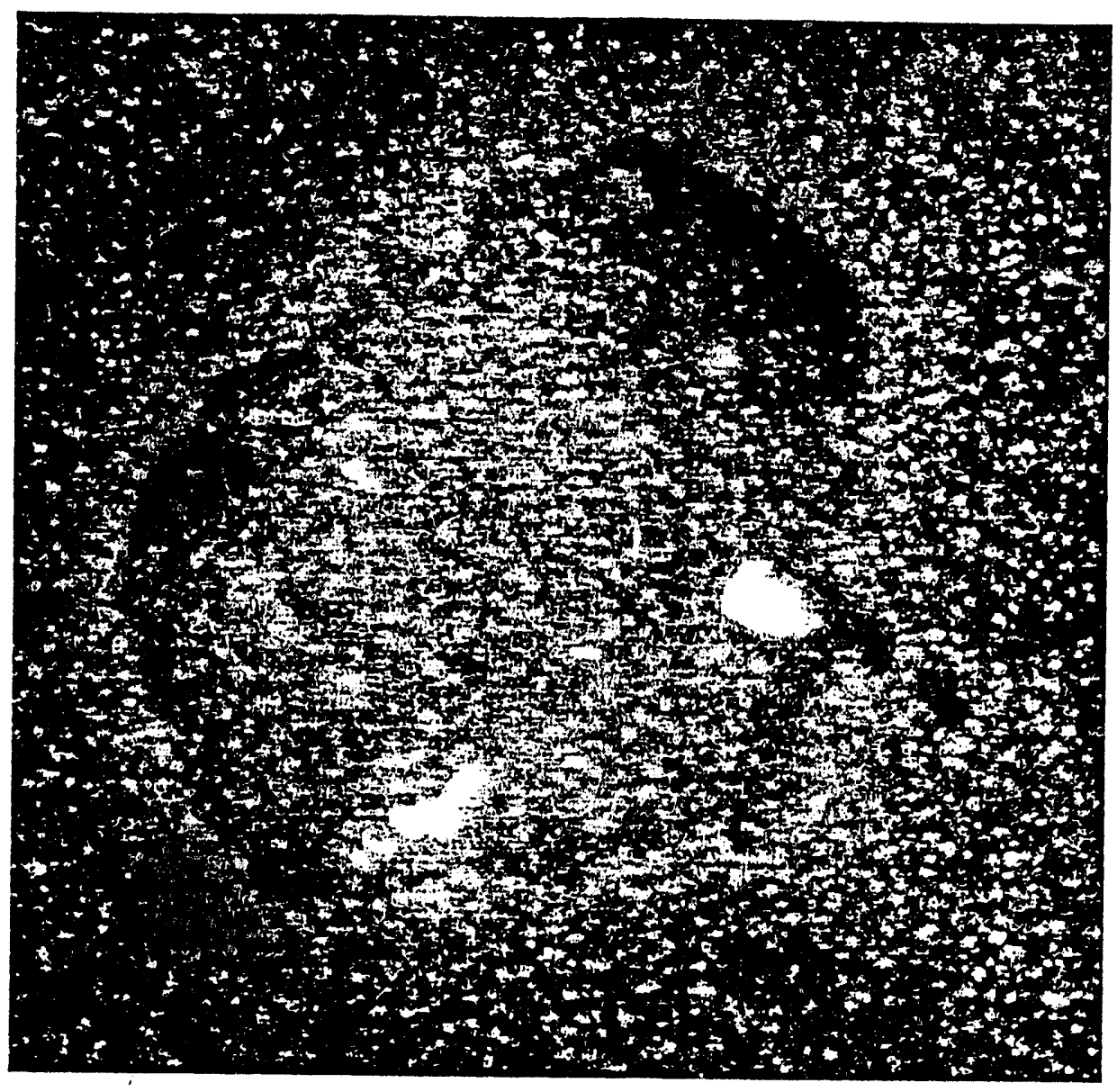

Figure 2. $1 \mathrm{~mm}$ "Spot" (approximately 95X). Note beginning of film grain structure to show. 
Acronyms and Abbreviations 


\section{Acronyms and Abbreviations}

$3 E$

AI

APS

AREST

ARM

ARRC

ATCC

AVHRR

BPDE

BWR

CALREL

CBE

CHEMFET

CHWI

COSL

CPMAS

CW

DC

DNA

DOD

DSC

DOE

DSOM

DTPA

ECMWF

ED3A

EDTA

EMF

EMSL

EPA

EPIC

EPR

ER\&D

FTICR

FTICR-MS

GC/ECD

GMDAS

HEDTA

HVAC

IAEA

IGEX energy, economics, environmental

artificial intelligence

Advanced Photon Source

Analytic Repository Source Term

Atmospheric Research Measurement

Advanced Recycling and Research Complexes

American Type Culture Collection

Advanced Very High Resolution Radiometer

benzo[a]pyrene diol epoxide

boiling water reactor

University of California, Berkeley, reliability

National Science Foundation Engineering Research Center for Biofilm Engineering

field effect transistor chemical sensor

Child Health and Welfare Index

cooled optically stimulated luminescence

cross polarized magic angle spinning

chemical weapons

direct current (dc)

deoxyribonucleic acid

U.S. Department of Defense

differential scanning calorimetry

U.S. Departmeut of Energy

Decision Support for Operations and Maintenance

diethylenetriaminepentacetic acid

European Centre for Medium-Range Weather Forecasts

ethylenediaminetriacetic acid

ethylenediaminetetraacetic acid

electromagnetic field

Environmental and Molecular Sciences Laboratory

U.S. Environmental Protection Agency

erosion productivity import calculator

electron paramagnetic resonance

Exploratory Research and Development

Fourier transform ion cyclotron resonance

Fourier transform ion cyclotron resonance mass spectroscopy

gas chromatograph/electron capture detector

global meteorological data analysis system

$\mathrm{N}$-(2-hydroxyethyl)ethylenediaminetriacetic acid

heating, ventilating, and air conditioning

International Atomic Energy Agency

International Germanium Experiment 
NR

IR

LOCA

LDRD

LST

LUAM

MARS

MEPAS

MFRSRs

MINK

MRC

MRM

MSRC

NCDC

NCSA

NIR

NIST

NMR

NSF

NTA

OOPLUAM

PAH

PASC

PCB

PBPK

PCNA

PNC

PNL

POMIS

PRESS

PWR

RTDS

RTUIS

SARAH

SGM

TEMPEST

TRU

TRUEX

USDA
Institute for Nuclear Research (Soviet Academy of Sciences)

infrared

loss-of-coolant-accident

Laboratory Directed Research and Development

local standard time

Land Use Allocation Model

multivariate adaptive regression splines

Multimedia Environmental Pollutant Assessment System

multiple filter rotating shadowband radiometers

Missouri, Iowa, Nebraska, and Kansas

Materials Research Center

modified reverse micelle

Molecular Science Research Center

U.S. National Climatic Data Center

National Center for Supercomputing Applications

non-ionizing radiation

National Institute of Standards and Technology

nuclear magnetic resonance

National Science Foundation

nitrilotriacetic acid

Object-Oriented Programming Land Use Allocation Model

polynuclear aromatic hydrocarbon

Pulse Amplitude Synthesis and Control

polychlorinated biphenyl

physiologically based pharmacokinetic

proliferating cell nuclear antigen

Pacific Northwest Consortium

Pacific Northwest Laboratory

portable operations and maintenance information system

process re-engineering support system

pressurized water reactor

Rapid Thermal Decomposition of precursors in Solution

Real-Time Ultrasonic Imaging System

Safety Analysis of Radiofrequency/Microwave Antenna Hazards Second Generation Model

Transient Energy Momentum and Pressure Equations Solutions transuranic

transuranium extraction

U.S. Department of Agriculture 


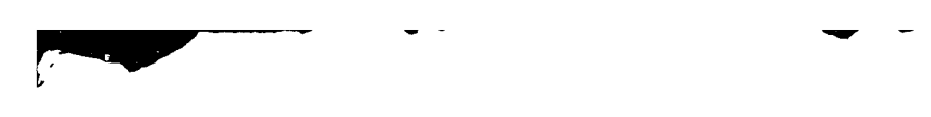
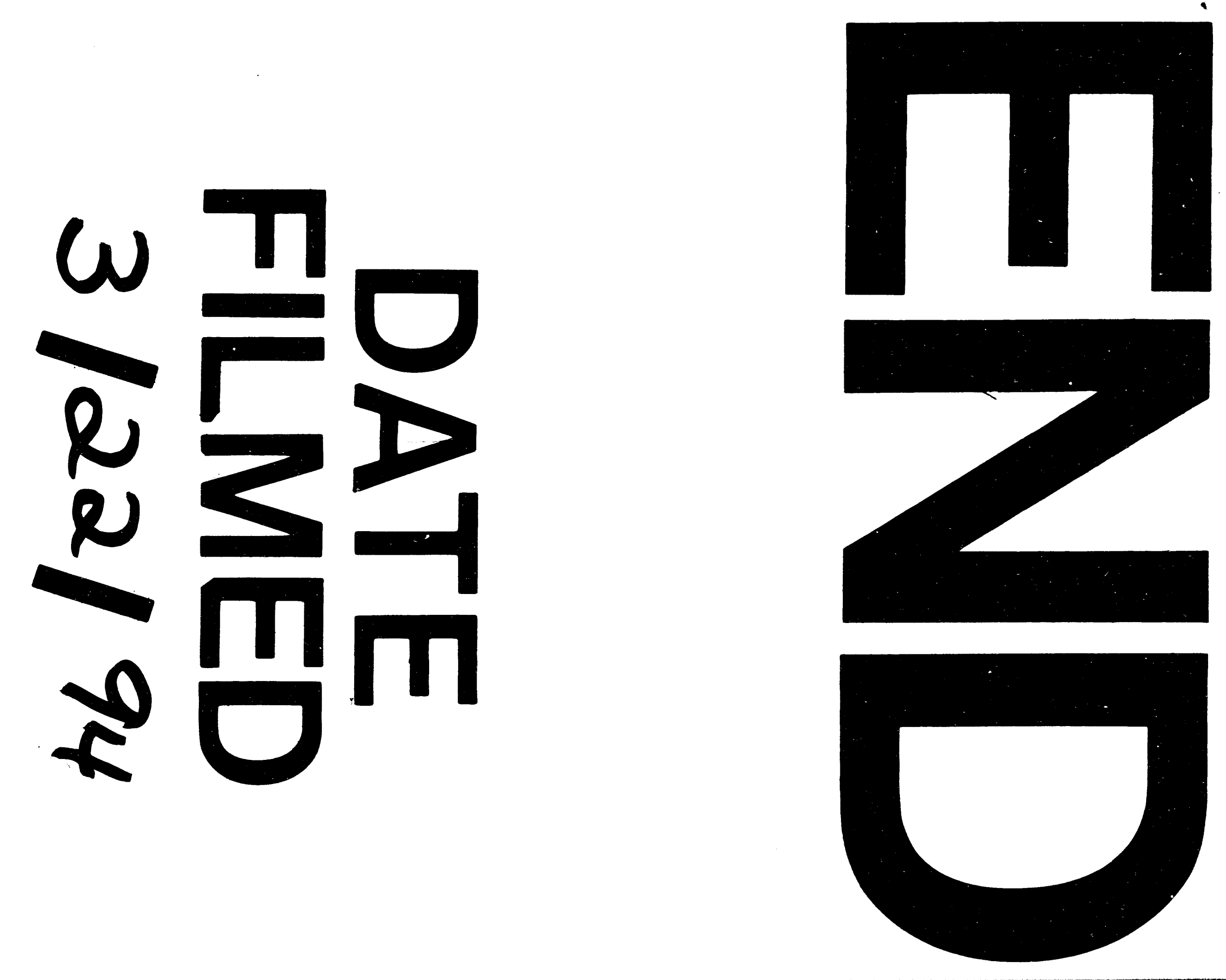

$A$ 


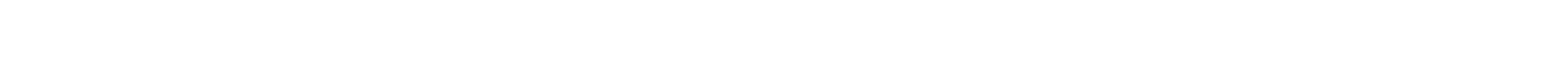
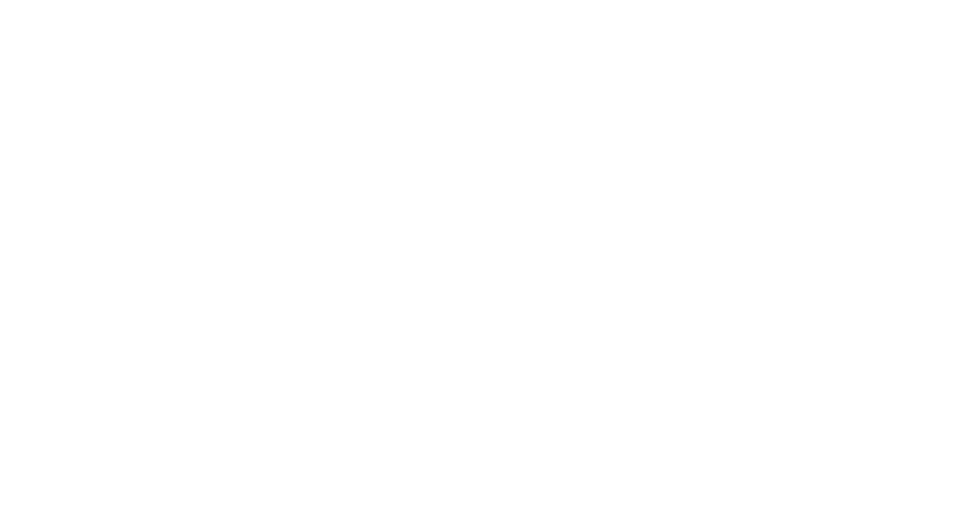

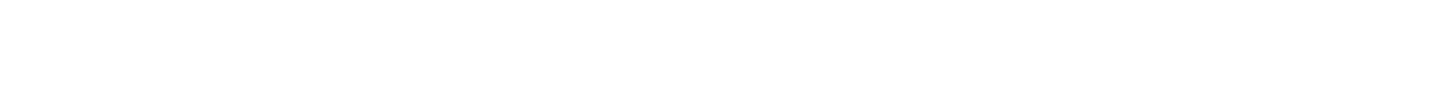

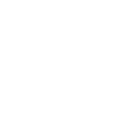
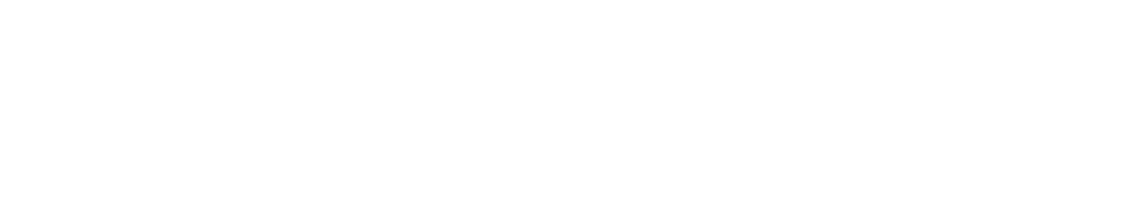